\title{
Aspects of Signal Processing in Noisy Neurons
}

\section{Dissertation}

zur Erlangung des Doktorgrades

der Mathematisch-Naturwissenschaftlichen Fakultäten

der Georg-August-Universität zu Göttingen

vorgelegt von

\section{Hans Ekkehard Pleßer}

aus Jülich

Göttingen 1999 
D7

Referent

Prof. Dr. Theo Geisel

Korreferent

Prof. Dr. Reiner Kree

Tag der mündlichen Prüfung: $\quad$ 1. November 1999 


\section{Kurzfassung}

\section{Signalverarbeitung in Neuronen unter dem Einfluß von Rauschen}

Das menschliche Gehirn ist das faszinierendste Rätsel der Naturwissenschaften. Mit einer Leistungsaufnahme von nur 12 Watt (Sarpeshkar 1998) ermöglicht uns dieses Organ, uns in unserer Umgebung zu orientieren, mit anderen Menschen zu kommunizieren und schließlich uns selbst als Denkende zu reflektieren. Auch die glänzendsten Erfolge der künstlichen Intelligenz, wie der Sieg des Schachcomputers Deep Blue über Weltmeister Garri Kasparow, verblassen vor dieser Leistung der Natur. Diese hat nun in Jahrmillionen steter Evolution mit unserem Gehirn ein Signalverarbeitungsorgan geschaffen, das sich verblüffend von klassischen Lösungen der Ingenieurkunst unterscheidet. So besteht das Gehirn aus einer ungeheueren Zahl scheinbar unzuverlässiger Nervenzellen, die in wunderbarer Weise zusammenwirken. Die aus der stochastischen Natur des Gehirns erwachsende, auf den ersten Blick hochgradig irreguläre Aktivität dieser Neurone hat Forscherinnen und Forscher immer wieder vor Rätsel gestellt.

Erst in jüngerer Zeit hat sich die Erkenntnis durchgesetzt, daß statistische Einflüsse, oft Rauschen genannt, die Verarbeitung von Signalen nicht notwendig behindern, sondern sogar unterstützen können. Dieser Effekt ist als stochastische Resonanz bekannt geworden (Benzi et al. 1981). Es liegt nahe, daß die Evolution Wege gefunden hat, dieses Phänomen zur Optimierung der Informationsverarbeitung bei minimalem Energiebedarf auszunutzen (Longtin et al. 1991; Laughlin et al. 1998). Die Informationsverarbeitung im Nervensystem scheint nun auf einem sorgfältig abgestimmten Wechselspiel von analoger Signalverarbeitung und digitaler Signalübertragung zu beruhen (Sarpeshkar 1998). Daher kommt dem Pulsgenerator des Neurons, der Schwankungen des über die Zellmembran des Neurons abfallenden Potentials als Pulsfolgen kodiert und an andere Neurone weiterleitet, eine besondere Bedeutung zu. Er ist gleichsam der Analog-Digital-Wandler der Zelle. Diese Dissertation untersucht, ob die Kodierung periodischer Signale im Pulsgenerator durch das ohnehin im Nervensystem vorhandene Rauschen verbessert wird, ob also stochastische Resonanz auftritt. 
Die Untersuchung wird am Beispiel des pulserzeugenden Integratorneurons mit Leckstrom durchgeführt (leaky integrate-and-fire neuron), welches sich durch drei wesentliche Eigenschaften auszeichnet: es reproduziert experimentelle Befunde hinreichend gut (siehe Kapitel 5.2), ist der mathematischen Analyse zugänglich und beschreibt die Erzeugung von Pulsfolgen und nicht etwa nur die zeitliche Änderung von Pulsraten. Letzteres ist von Bedeutung, da eine wachsende Zahl experimenteller Arbeiten nahelegt, daß die präzise zeitliche Struktur der Pulsfolgen ein wesentlicher Bestandteil der neuronalen Kodierung ist (Rieke et al. 1997). Das pulserzeugende Integratorneuron und seine engsten "Verwandten" werden daher weithin zum Studium der neuronalen Kodierung verwendet (Gerstner 1999b). Das Rauschen wird in dieser Arbeit als gaussisch und weiß angenommen, so daß die zeitliche Entwicklung des Membranpotentials des Neurons durch einen Ornstein-Uhlenbeck Prozeß beschrieben wird. Das Modell wird daher als Ornstein-Uhlenbeck Neuron bezeichnet (Uhlenbeck and Ornstein 1930; Lánský and Rospars 1995). Das Problem, die Verteilung der Intervalle zwischen zwei Pulsen zu bestimmen (interspike-interval distribution, ISID), ist damit genau das Erstpassagezeitproblem für den Ornstein-Uhlenbeck Prozeß mit einem absorbierenden Rand (Schrödinger 1915). Alle hier erzielten Ergebnisse sind daher direkt auf Ornstein-Uhlenbeck Prozesse beliebigen Ursprungs übertragbar.

Da das digitale Ausgangssignal des Neurons aus einer Folge stereotyper Pulse besteht (spike train), trägt nicht die Form der Pulse, sondern lediglich ihre Anordnung in der Zeit Bedeutung. Deshalb werden die vom Neuronenmodell erzeugten Pulsfolgen mit den Methoden der Theorie der Punktprozesse untersucht (Cox and Lewis 1966). Als Maß für die Güte der Kodierung eines periodischen Signals wird das Signal-Rausch-Verhältnis der erzeugten Pulsfolge verwendet (signal-to-noise ratio, SNR).

Das wesentliche Ergebnis dieser Dissertation ist, daß das Ornstein-Uhlenbeck Neuron zwei Arten rauschinduzierter Resonanz zeigt: zum einen klassische stochastische Resonanz, d.h. ein optimales Signal-Rausch-Verhältnis bei einer bestimmten Amplitude des Eingangsrauschens. Hinzu tritt eine Resonanz bezüglich der Frequenz des (deterministischen) Eingangssignals, des Reizes. Reize eines bestimmten Frequenzbereichs werden somit in Pulsfolgen kodiert, die zeitlich deutlich strukturiert sind, während Stimuli außerhalb des bevorzugten Frequenzbandes zeitlich homogenere Pulsfolgen auslösen. Für diese zweifache Resonanz wird der Begriff stochastische Doppelresonanz eingeführt (stochastic double resonance, SDR). Der Effekt wird auf elementare Mechanismen zurückgeführt und seine Abhängigkeit von den Eigenschaften des Reizes umfassend untersucht. Dabei zeigt sich, daß die Reizantwort des Neurons einfachen Skalengesetzen unterliegt und von den verbleibenden skalierten Größen entweder unabhängig ist oder aber in einfacher Weise abhängt. Insbesondere ist die optimale skalierte Rauschamplitude ein universeller Parameter des Modells, der vom Reiz gänzlich unabhängig zu sein scheint. Die optimale Reizfrequenz hängt hingegen linear von der skalierten Reizamplitude 
ab, wobei die Proportionalitätskonstante wiederum vom Gleichstromanteil des Reizes bestimmt wird (Basisstrom, base current). Während große Basisströme Frequenz und Amplitude nahezu entkoppeln, so daß Reize beliebiger Amplitude in zeitlich wohlstrukturierten Pulsfolgen kodiert werden, erlauben es kleine Basisströme, das optimale Frequenzband durch Veränderung der Reizamplitude zu wählen. Da Pulspakete nur dann durch ein neuronales Netzwerk propagieren können, wenn sie genügend groß und zeitlich hinreichend scharf sind (Diesmann et al. 1999), könnte die stochastische Doppelresonanz der selektiven Signalverarbeitung mit Hilfe sogenannter Synfire-Ketten zugrundeliegen (Abeles 1991).

Als ein weiteres Ergebnis wird gezeigt, daß stochastische Resonanz lediglich als Artefakt einer kaum haltbaren Modellannahme auftritt, wenn zur Vereinfachung der mathematischen Behandlung vorausgesetzt wird, daß der Reiz nach jedem vom Neuron abgefeuerten Puls auf eine feste Phase zurückgesetzt wird (Reizung mit Nebenbedingung, constrained stimulation).

Diese Ergebnisse zur Signalverarbeitung fußen auf einigen methodischen Neu- bzw. Fortentwicklungen, die insbesondere alle diejenigen Fragen beantworten, die Gammaitoni et al. (1998, Sec. V.C.4) in einem unlängst erschienenen Übersichtsartikel aufwerfen. Zunächst wird ein zuverlässiges numerisches Verfahren zur Bestimmung der Intervallängenverteilung entwickelt. Dieses wird ergänzt durch verbesserte und erstmals quantitativ geprüfte Näherungen für diese Verteilungen. Während die Lösungen des Erstpassagezeitproblems des Ornstein-Uhlenbeck Prozesses nur in impliziter Form vorliegen, ermöglichen diese Näherungen, speziell das Arrheniusmodell mit Stromterm (Arrhenius\&Current model), analytische Untersuchungen in in weit größerem Umfang. Zur Analyse von Pulsfolgen, die von periodischen Pulsfolgen ohne Rücksetzen erzeugt werden (unconstrained stimulation), wird eine Markoffkettenanalyse entwickelt, die es insbesondere erlaubt, das Leistungsspektrum der Pulsfolge und damit deren Signal-Rausch-Verhältnis zu bestimmen. Ebenso kann die Pulsintensität (spike intensity) berechnet werden, die in der Vergangenheit wenig Beachtung gefunden hat, obwohl sie ausgezeichnet geeignet ist, um die Reizantwort des Neurons zu veranschaulichen. Für den Fall der Reizung mit Nebenbedingung werden einige Methoden aus der Theorie der Erneuerungsprozesse abgeleitet. Programmcode für alle neu entwickelten Verfahren wird öffentlich zugänglich gemacht (Plesser 1999).

Die Dissertation gliedert sich wie folgt: Kapitel 1 gibt einen ausführlichen Überblick über die Problemstellung, bevor das Ornstein-Uhlenbeck Neuron in Kapitel 2 eingeführt wird. Näherungen für die Intervallverteilung werden in Kapitel 3 untersucht, während in Kapitel 4 die Markoffkettenmethode entwickelt und geschlossene Ausdrücke für die spektrale Leistungsdichte und das Signal-Rausch-Verhältnis abgeleitet werden. Die Ergebnisse dieser Dissertation sind in Kapitel 5 dargestellt und werden in Kapitel 6 zusammenfassend diskutiert. Zwei Anhänge behandeln technische Aspekte. 



\section{Contents}

German Abstract

List of Figures $\quad \mathrm{x}$

List of Tables $\quad$ xi

List of Symbols $\quad$ xiii

1 Introduction 1

2 The Ornstein-Uhlenbeck Neuron $\quad 9$

2.1 Derivation from Stein's model . . . . . . . . . . . . . . . 11

2.1.1 Fokker-Planck equation . . . . . . . . . . . . . 12

2.1.2 Diffusion requires inhibition . . . . . . . . . . . . . . 14

2.1.3 Canonical form of the Ornstein-Uhlenbeck neuron . . . . 16

2.2 Interspike-interval density . . . . . . . . . . . . . . . . . . . 17

2.2.1 Elementary properties . . . . . . . . . . . . . 18

2.2.2 Schrödinger's renewal Ansatz . . . . . . . . . . . 20

2.2.3 Strict positivity of the ISI density . . . . . . . . . . . 22

2.3 Stimuli . . . . . . . . . . . . . . . . . . . 23

3 Approximating the Ornstein-Uhlenbeck Neuron 27

3.1 Hazard models . . . . . . . . . . . . . . . . . 27

3.1.1 Arrhenius model . . . . . . . . . . . . . . . 28

3.1.2 Arrhenius\&Current model . . . . . . . . . . . . . . 30

3.1 .3 Abeles model . . . . . . . . . . . . . . . . . . 32

3.1.4 Linear ramp model . . . . . . . . . . . . . . . . . . 33

3.1.5 Tuckwell model . . . . . . . . . . . . . . . . 33

3.2 Method-of-images approximation . . . . . . . . . . 34

3.3 Evaluation . . . . . . . . . . . . . . . . 35

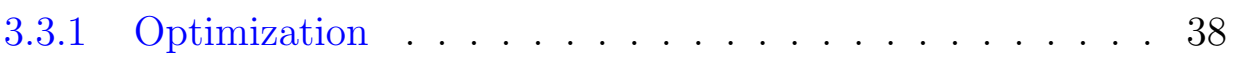

3.3.2 Performance . . . . . . . . . . . . . . . . 38

3.4 Summary . . . . . . . . . . . . . . . . . . . . . 42 
4 Spike Trains $\quad 45$

4.1 Definitions . . . . . . . . . . . . . . . . 46

4.2 Approximate renewal trains . . . . . . . . . . . . . . . . . . 49

4.3 Constrained stimulation . . . . . . . . . . . . . . 51

4.4 Unconstrained stimulation . . . . . . . . . . . . . . 54

4.4.1 Continuous phase: Markov kernel . . . . . . . . . . . 55

4.4.2 Discrete phase: Markov chain . . . . . . . . . . . . . 58

4.4.3 Markov chain in time . . . . . . . . . . . . . 61

4.5 Spike train statistics . . . . . . . . . . . . . . . . . . . . . . . . . . . . . 63

4.6 Power spectral density . . . . . . . . . . . . . . . . 65

4.6.1 PSD for constrained stimulation . . . . . . . . . . 68

4.6.2 PSD at harmonics of unconstrained stimuli . . . . . . . 71

4.6.3 Full PSD for unconstrained stimulation . . . . . . . . . 77

4.7 Signal-to-noise ratio . . . . . . . . . . . . . . . . . 79

5 Noise Aided Signal Processing $\quad 81$

5.1 Constrained stimulation . . . . . . . . . . . . . . 82

5.2 Unconstrained stimulation: stationary response . . . . . . . . . 90

5.3 Stochastic double resonance . . . . . . . . . . . . . . 97

5.3 .1 Mechanism . . . . . . . . . . . . . . . 100

5.3.2 Role of stimulus amplitude and base current . . . . . . . . 103

5.3.3 Stochastic resonance in the cortical regime . . . . . . . . 111

$\begin{array}{lll}6 & \text { Summary } & 115\end{array}$

A Numerical Evaluation of First-Passage-Time Densities 121

A.1 Algorithm . . . . . . . . . . . . . . . . . . . . 121

A.2 Validation . . . . . . . . . . . . . . . . . 124

$\begin{array}{lr}\text { B Spike Train Simulation } & 127\end{array}$

B.1 Algorithm . . . . . . . . . . . . . . . . . . . 127

B.2 Validation . . . . . . . . . . . . . . . . . . . 129

$\begin{array}{ll}\text { References } & 131\end{array}$

$\begin{array}{lr}\text { Publications } & 143\end{array}$

$\begin{array}{ll}\text { Acknowledgments } & 145\end{array}$

$\begin{array}{lr}\text { Curriculum Vitae } & 147\end{array}$ 


\section{List of Figures}

2.1 Leaky integrate-and-fire neuron . . . . . . . . . . . . . . . 10

2.2 Schrödinger's renewal Ansatz . . . . . . . . . . . . . . 21

3.1 Hazard functions: overview . . . . . . . . . . . . . . . . . . . . . . 29

3.2 Hazard: Arrhenius model . . . . . . . . . . . . . . . . 30

3.3 Hazard: Arrhenius\&Current model . . . . . . . . . . . . . . . 31

3.4 Hazard: Abeles model . . . . . . . . . . . . . . . . . . 32

3.5 ISI density: approximations vs. OU neuron . . . . . . . . . . . 36

3.6 Optimization of model parameters . . . . . . . . . . . . . . 37

3.7 Approximation error vs. distance from threshold . . . . . . . . . 39

3.8 Error of the method-of-images approximation . . . . . . . . . . 40

3.9 Error of hazard models . . . . . . . . . . . . . . . . . . 41

3.10 Arrhenius vs. Tuckwell model . . . . . . . . . . . . . . . . . 42

3.11 Cumulative distribution of errors . . . . . . . . . . . . 43

4.1 Validity of the renewal approximation . . . . . . . . . . . 50

4.2 Constrained stimulation: effective stimulus . . . . . . . . . . . 52

4.3 Spike intensity: constrained stimulation . . . . . . . . . . . 53

4.4 Construction of the Markov kernel . . . . . . . . . . . . . . . 56

4.5 Markov chain: firing phase evolution . . . . . . . . . . . . 60

4.6 Spike intensity: unconstrained stimulation . . . . . . . . . . . 62

4.7 Count-conditional joint spike time density . . . . . . . . . . . 67

4.8 Power spectral density: constrained stimulation . . . . . . . . . 69

4.9 Count-conditional joint spike phase density . . . . . . . . . . 71

4.10 Power spectral density: unconstrained stimulation . . . . . . . . 74

4.11 Error of Markov chain PSD approximation . . . . . . . . . . 75

4.12 Power spectral density vs. observation time . . . . . . . . . 76

4.13 Full power spectral density approximation . . . . . . . . . 78

5.1 Constrained stimulation: response properties . . . . . . . . 83

5.2 Constrained stimulation: effect of initial phase . . . . . . . . . 85

5.3 Constrained stimulation: stochastic resonance . . . . . . . . . 86

5.4 Constrained stochastic resonance: mechanism . . . . . . . . 87

5.5 ISI density shift with noise amplitude . . . . . . . . . . . . 88 
5.6 Noise-adapted phase: no stochastic resonance . . . . . . . . . . 89

5.7 Unconstrained stimulation: firing patterns . . . . . . . . . . . . . 91

5.8 Unconstrained stimulation: transition kernels . . . . . . . . . 92

5.9 ISI density, spike phase density and spike intensity . . . . . . . . 93

5.10 OU neuron vs. monkey auditory nerve fiber . . . . . . . . . . 94

5.11 Cold receptor responses in cat . . . . . . . . . . . . . . 9 96

5.12 Modeling cold receptor responses . . . . . . . . . . . . . . . . . . . . . . . . . . 97

5.13 Power spectral density: examples . . . . . . . . . . . . . . 98

5.14 Stochastic double resonance . . . . . . . . . . . . . . . . . . 99

5.15 Stochastic resonance: quantity vs. quality . . . . . . . . . . 101

5.16 Bona fide resonance: timescale matching . . . . . . . . . . . . 102

5.17 Signal-to-noise ratio: parameter dependence . . . . . . . . . . 104

5.18 Parameters governing neuron response properties . . . . . . . 106

5.19 Optimal signal-to-noise ratio . . . . . . . . . . . . . . 107

5.20 Signal-to-noise ratio: Arrhenius\&Current model . . . . . . . . 108

5.21 Signal-to-noise ratio: scaled noise amplitude . . . . . . . . . . 109

5.22 Optimal transition matrices . . . . . . . . . . . . . . 110

5.23 Stochastic double resonance: cortical regime . . . . . . . . . . . 112

5.24 Optimal signal-to-noise ratio: cortical regime . . . . . . . . . . 113

5.25 Optimal frequency: dependence on stimulus . . . . . . . . . . 114

B.1 Test of spike train simulation _. . . . . . . . . . . . . 129 


\section{List of Tables}

3.1 Error of approximations to the OU neuron . . . . . . . . . . 43

4.1 Spike train statistics . . . . . . . . . . . . . . . . 64

5.1 Standard stimulus set . . . . . . . . . . . . . . . 82

A.1 Error of block-by-block method: constant input . . . . . . . . 124

A.2 Error of block-by-block method: periodic input . . . . . . . 125

B.1 Simulated spike trains: goodness of fit . . . . . . . . . 130 



\section{List of Symbols}

\begin{tabular}{|c|c|c|}
\hline$\lceil x\rceil$ & $\min \{n \in \mathbb{Z} \mid x \leq n\}$ & \\
\hline$\lfloor x\rfloor$ & $\max \{n \in \mathbb{Z} \mid n \leq x\}$ & \\
\hline$[x]_{+}$ & $\frac{1}{2}(x+|x|)$ & \\
\hline$\langle x\rangle$ & expectation of random variable $x$ & \\
\hline$\|x\|$ & $L^{1}$-norm of $x$ & \\
\hline$O(x)$ & $\lim _{x \rightarrow 0}\left|\frac{O(x)}{x}\right|<\infty$ & \\
\hline$o(x)$ & $\lim _{x \rightarrow 0} \frac{o(x)}{x}=0$ & \\
\hline $\operatorname{atan} x$ & arc tangent, $\operatorname{atan} x \in(-\pi / 2, \pi / 2)$ & \\
\hline$\chi_{k}(\psi)$ & spike phase density (SPD) & p. 55 \\
\hline$\chi^{(s)}(\psi)$ & stationary spike phase density & p. 57 \\
\hline$\chi_{k}(\psi \mid \phi)$ & conditional spike phase density (cSPD) & p. 57 \\
\hline$\chi_{j+k, j}\left(\psi, \phi \mid T_{o}, M\right)$ & spike-count-conditional joint SPD & p. 72 \\
\hline$C_{\mathrm{v}}$ & coefficient of variation & p. 64 \\
\hline $\operatorname{diag}\left(x_{1}, \ldots, x_{n}\right)$ & diagonal matrix with elements $x_{1}, \ldots, x_{n}$ & \\
\hline$E$ & relative integrated mean square error (rIMSE) & p. 35 \\
\hline$\varepsilon$ & relative distance from threshold & p. 25 \\
\hline $\operatorname{erf} x$ & error function, $\frac{2}{\sqrt{\pi}} \int_{0}^{x} \mathrm{e}^{-w^{2}} \mathrm{~d} w$ & \\
\hline $\operatorname{erfc} x$ & $1-\operatorname{erf}(x)$ & \\
\hline$\eta^{2}(\tau)$ & variance of membrane potential density & p. 20 \\
\hline $\bar{f}(s)$ & Laplace transform $\bar{f}(s)=\int_{0}^{\infty} f(t) \mathrm{e}^{-s t}$ & \\
\hline$\tilde{f}(\omega)$ & Fourier transform $\tilde{f}(\omega)=\int_{-\infty}^{\infty} f(t) \mathrm{e}^{-i \omega t}$ & \\
\hline$f_{\mathbf{t}}(t)$ & output of neuron & p. 10 \\
\hline$h(t \mid I(t), \hat{t})$ & hazard function & p. 28 \\
\hline$I(t)$ & stimulus & p. 23 \\
\hline$\mu$ & base current of stimulus & p. 23 \\
\hline $\operatorname{nrm}(m, s)$ & normal random number, mean $m$, variance $s$ & p. 128 \\
\hline$\Omega$ & stimulus frequency & p. 24 \\
\hline
\end{tabular}




\begin{tabular}{|c|c|c|}
\hline$\Omega_{c}$ & cut-off frequency, aperiodic stimuli & p. 24 \\
\hline $\mathcal{P}(v, t \mid w, s)$ & membrane potential density & p. 12 \\
\hline $\mathcal{P}_{f}(v, t \mid w, s)$ & $\begin{array}{l}\text { membrane potential density, } \\
\text { free boundary conditions }\end{array}$ & p. 20 \\
\hline $\mathcal{P}_{h}(v, t \mid w, s)$ & $\begin{array}{l}\text { approximate membrane potential density, } \\
\text { free boundary conditions }\end{array}$ & p. 28 \\
\hline$p_{N}(T)$ & spike count distribution for time $T$ & p. 65 \\
\hline$q$ & stimulus amplitude & p. 23 \\
\hline$q_{r}$ & relative stimulus amplitude & p. 106 \\
\hline$\hat{q}_{r}$ & dampened relative stimulus amplitude & p. 106 \\
\hline$q(t)$ & spike intensity & p. 47 \\
\hline$q_{k}(t)$ & spike time density (STD) & p. 47 \\
\hline$q_{j, k}(t, s)$ & joint spike time density (jSTD) & p. 47 \\
\hline$q_{k}(t \mid s)$ & conditional spike time density (cSTD) & p. 47 \\
\hline$q_{j+k, j}\left(t, s \mid T_{o}, M\right)$ & spike-count-conditional jSTD & p. 67 \\
\hline$r$ & vector strength & p. 64 \\
\hline$\rho(\tau)$ & interspike-interval density (renewal process) & p. 51 \\
\hline$\rho(\tau \mid \hat{t})$ & conditional ISI density (cISID) & p. 18 \\
\hline$\rho^{(s)}(t)$ & stationary interspike-interval density & p. 58 \\
\hline $\operatorname{rk}(x)$ & rank of $x$ in ordered set & p. 49 \\
\hline$\sigma$ & noise amplitude & p. 16 \\
\hline$\sigma_{r}$ & relative noise amplitude & p. 106 \\
\hline$S_{T}(\omega)$ & power spectral density (PSD) for time $T$ & p. 66 \\
\hline$S_{T, M}(\omega)$ & spike-count-conditional PSD & p. 66 \\
\hline SNR & signal-to-noise ratio & p. 80 \\
\hline$\hat{t}$ & time of most recent spike & p. 18 \\
\hline t & spike train, i.e. ordered list of spike times & p. 10 \\
\hline$T(\psi \mid \phi)$ & kernel of Markov chain in phase & p. 55 \\
\hline $\mathcal{T}$ & operator of Markov chain in phase & p. 56 \\
\hline$T_{o}$ & observation time & p. 65 \\
\hline$\langle\tau\rangle$ & mean interspike interval & p. 64 \\
\hline$v_{0}(t ; w, s)$ & noise-free membrane potential & p. 20 \\
\hline$\Upsilon_{n}(\mathbf{t})$ & spike train density & \\
\hline
\end{tabular}




\section{Chapter 1}

\section{Introduction}

The human brain is the most fascinating puzzle posed to the scientist. With a minute power consumption of just 12 watt (Sarpeshkar 1998), the nervous system provides us with a highly efficient signal processing, memory and control mechanism. Even more, this system can adapt to environmental conditions on short timescales - we are able to learn - and has an astonishing ability to re-organize itself. For example, the auditory cortex may process sign language in deaf humans (Nishimura et al. 1999). Above all, the brain provides us with the most unfathomable qualities: a free will, a conscience and the conscious awareness of our own existence. ${ }^{1}$

Technology has little to put up against this feat of evolution: there might be sensors more suitable for night vision, number crunching computers excelling in banking and simulations, and CD-ROM archives less fallible than the memory of a human being. But even marveled achievements of artificial intelligence, such as the victory of Deep Blue over chess world champion Garry Kasparov ought to be put in proportion: a power consumption of several kilowatt, plus an operating team, versus a single brain running on but a dozen watt. Besides, Deep Blue is good for little but playing chess, while Garry Kasparov might well crack a joke or treat you to a cup of tea.

The brain is the outcome of a billion years of optimization in the presence of ecological constraints: not guaranteed to be best, but certainly impressive. This optimization has created a solution surprisingly different from all machinery engineers have designed till very recently. ${ }^{2}$ Whereas the latter strove to retain control over all aspects of their inventions - and a nuclear power plant should better be under control at all times - nature forwent total control to achieve better. Evolution created a fascinating ensemble of independent,

\footnotetext{
${ }^{1}$ From a mechanistic viewpoint, the free will is the ultimate achievement: a deterministic apparatus persuading itself of a freedom it does not possess - and providing the means to uncover, after some three millenia of philosophy and science, this paradox. In short, the material triumph of mind over matter.

${ }^{2}$ The same can be said for chemistry: the cellular machinery far excels organic chemistry in the synthesis of proteins.
} 
seemingly fallible neurons which miraculously interact to form a working brain. The ensuing highly irregular activity has puzzled neuroscientists from the early days on.

This irregularity has commonly been declared noise, considered a nuisance, and averaged out over repetitions of experiments. But are we indeed to accept that hundreds of millions of years of evolution would have culminated in a "noisy" brain if a system void of irregularity were better? To the contrary, we ought to conclude that the brain benefits from irregularity. This idea has been gaining acceptance in recent years (Laughlin et al. 1998).

The task is thus posed to elucidate how such irregularity, be it from fluctuating receptor-cell responses, from synaptic transmission, or from uncorrelated signals, affects the transmission of a signal through an individual neuron. This dissertation aims to contribute to this task, with a focus on the transmission of periodic signals by neurons of the sensory pathways of vertebrates.

\section{The benefits of noise: Stochastic resonance}

Based on geophysical observations, Benzi, Sutera, and Vulpiani (1981) suggested that minute periodic variations of the orbit of the earth might regularly induce ice ages by virtue of noise-induced resonance, an effect they called stochastic resonance. It has been established firmly in a large number of physical systems since and is well understood theoretically. A comprehensive review is given by Gammaitoni et al. (1998), while Wiesenfeld and Jaramillo (1998) provide a concise summary oriented towards applications in biology.

A system is said to exhibit stochastic resonance if the proper amount of input noise induces an optimal coherence of output and input signal. To investigate stochastic resonance, experimentalists usually feed a deterministic signal into the system under study, such as Schmitt triggers (Fauve and Heslot 1983), lasers (McNamara et al. 1988; Giacomelli et al. 1999), level crossing detectors (Gingl et al. 1995), or ion channels (Bezrukov and Vodyanoy 1997). Some response property is measured, including the power spectral density (Benzi et al. 1981), the signal-to-noise ratio (McNamara et al. 1988), the correlation to the input signal (Collins et al. 1995), the transinformation (Levin and Miller 1996), or residence time distributions (Gammaitoni et al. 1989). If this response property passes through a maximum for a particular amplitude of the input noise, this is taken as evidence for stochastic resonance.

The theory of stochastic resonance was first developed for dynamical systems, which permit analytical approaches, although most of this work is limited to the adiabatic regime for technical reasons (McNamara and Wiesenfeld 1989). This precludes the investigation of the influence of the signal frequency on the stochastic resonance effect. The appearance of stochastic resonance can roughly be explained as follows: The system will generate discernible output every time its internal state surmounts some barrier. If the deterministic input 
signal is too weak to induce crossings, the system will be silent in the absence of noise, and weak noise will induce only rare, incoherent crossings. Strong noise, on the other hand, will induce frequent, but random transitions. At an intermediate noise intensity, though, the rate of the noise-induced crossings will coincide with the timescale set by the input signal, yielding a coherent output signal. Stochastic resonance is thus a cooperative effect between signal and noise.

Longtin, Bulsara, and Moss (1991) were the first to search for stochastic resonance in the nervous system. Since then, it has been demonstrated in sensory modalities of many species, including crayfish mechanoreceptors (Douglass et al. 1993), the cricket cercal system (Levin and Miller 1996), rat cutaneous receptors (Collins et al. 1996), human muscle spindles (Cordo et al. 1996), hair cells of the inner ear (Jaramillo and Wiesenfeld 1998), and the human visual system (Simonotto et al. 1997; Srebro and Malladi 1999). Russell and Moss (1998) have recently reported evidence for the behavioral relevance of stochastic resonance: young paddlefish living in the murky waters of the Mississippi river can detect their plankton prey much better in the presence of (electric) noise. ${ }^{3}$ The application of stochastic resonance to improve cochlear implants for the profoundly deaf appears to be well on its way (Morse and Evans 1996).

Early theoretical studies of stochastic resonance in neurons were based on two-state neurons as suggested by McCulloch and Pitts (1943), which could be treated in the framework of the theory for bistable systems (Bulsara et al. 1991). Work on more realistic neuron models such as the FitzHugh-Nagumo model relied on simulations (Longtin 1993; Wiesenfeld et al. 1994). Other authors treated neurons as simple threshold detectors, ignoring the singular perturbation induced by spikes (Jung 1994), or as rate coders (Collins et al. 1996). The investigation of more tractable integrate-and-fire neuron models has suffered from technical difficulties and has been limited to slow stimuli (Bulsara et al. 1994; Gitterman and Weiss 1995; Bulsara et al. 1996). Gammaitoni et al. summarize the situation as follows in their review (1998, Sec. V.C.4):

As yet, the theory above is based on a number of unrealistic assumptions; moreover, it contains technical difficulties that have yet to be overcome:

(1) The phase of the sinusoidal stimulus has been reset after each firing event to the same initial value. This approximation is unrealistic from a physiological point of view, since a large amount of information about the coherence of the stimulus is eliminated. A theory of first-passage time distributions in the presence of a periodic forcing that explicitly avoids this assumption has not yet been put forward.

\footnotetext{
${ }^{3}$ For more on the electrosensory system of the paddlefish, see Wilkens et al. (1997).
} 
(2) Since the resting voltage of a neuron is very close to the potassium voltage, being a lower bound for the variation of the membrane voltage, an originally sinusoidal stimulus becomes strongly rectified. It is therefore not realistic simply to add the sinusoidal stimulus to the membrane voltage in the integrateand-fire model without taking into account rectification.

(3) Strictly speaking, the method of image sources [used by Bulsara et al. (1996) to obtain the interspike-interval density] is applicable only to diffusion processes that are homogeneous in space and time variables. The error made by using this method (as an approximation) in time-inhomogeneous equations ... has not been estimated mathematically.

Methods for the analysis of periodic forcing without reset are presented in Chapter 4 answering to (1). Chapter 5.2 provides experimental evidence that rectification of stimuli as mentioned in (2) may safely be ignored. The methodof-images approximation of Bulsara et al. (1996) is tested quantitatively in Chapter 3 and shown to be highly unreliable, confirming the doubts raised in (3). Better approximations are proposed.

\section{Origins of noise in the nervous system}

Before embarking on a study of the effect of noise on the workings of neurons, the origin of this noise deserves some comment. In most physical systems, such as the archetypical Brownian motion of lycopodium spores on water, clearly separated timescales exist as well as established methods to distinguish the slow, macroscopic degrees of freedom from the fast ones, which are treated as noise (van Kampen 1985; Eyink 1998). The situation is less clear in the neurosciences, where three sources of irregularity are to be considered: the membrane, the synapses, and uncorrelated signals. ${ }^{4}$

The membrane of neurons is a lipid bilayer, nearly impenetrable to most ions, with interspersed ion channels and pumps, generating an electric potential difference between the interior and the exterior of the cell. Both the gating of channels and the pumping of ions are discrete processes, giving rise to fluctuations. The sheer number - and supposed independence - of channels and pumps averages these fluctuations to a level where they are largely irrelevant to the operation of the neuron. Indeed, the great success of models based

\footnotetext{
${ }^{4}$ Further sources are changes in the pattern of innervation and direct electromagnetic influences of the environment on neurons. The former preclude direct comparison across individuals of a species. Fortunately, the rewiring of neurons is sufficiently slow to be neglected in studies on neural information processing which do not focus on learning. The latter - while hotly debated in the context of electro-smog — seems to be a speculative issue, even though it is exploited in some experiments (Gluckman et al. 1996), as well as by specialized species (Neiman et al. 1999); but see Holt and Koch (1999).
} 
on channel kinetics, such as the Hodgkin-Huxley and Morris-Lecar models, in describing the response of isolated neurons to injected currents testifies to the irrelevance of fluctuations arising from the discreteness of channels (Kandel et al. 1991; Ermentrout 1996). This conclusion holds likewise for the somatic spike generator (Bryant and Segundo 1976; Mainen and Sejnowski 1995). The membrane is thus no relevant source of irregularity in firing patterns.

Genuine irregularity is generated at chemical synapses: each signal transmission between pre- and postsynaptic cell is achieved by the release of a random number of practically identical neurotransmitter vesicles (Johnston and Wu 1995). This results in measurable fluctuations of postsynaptic currents and potentials. As the number of vesicles released has a Poisson distribution, this may be likened to radioactive decay as a true source of randomness. ${ }^{5} \mathrm{~A}$ recent study by Maass and Natschläger (1999) indicates that this synaptic unreliability may permit pools of neurons to perform analog computations.

The major source of irregularity in neuronal activity are not fluctuations at the molecular or synaptic level: Mainen and Sejnowski (1995) have demonstrated that the irregularity of firing patterns of cortical neurons can largely be traced to the irregularity of the input impinging on these neurons. This is consistent with the observation that cortical neurons fire highly randomly when recorded from the living animal, while generating regular spike sequences in slice preparations (Stevens and Zador 1998). The irregularity of neuronal spike trains in vivo may be explained as follows: except for specific neurons in sensory or motor pathways, only a small proportion of all inputs to a neuron will transmit information related to the specific stimulus presented by the experimentalist. Given the energy-efficiency of the brain, it appears highly implausible that all other input will just be nonsensical: it will convey some other information. It is by definition of the experimental task that these signals are termed noise.

\section{Spiking neuron models}

Most neurons in the central nervous system may be subdivided into a dendritic tree, a cell body (soma), and an axon. Input from other neurons arrives at tens of thousands of synapses located on the branches of the dendritic tree in form of miniature current pulses. These are summed across the tree, yielding a net input current which induces variations of the potential difference across the cell membrane in the soma. If this potential reaches a threshold, a sharp voltage pulse - an action potential or spike-is generated at the axon hillock, a particularly excitable patch of the membrane. This spike rapidly propagates along the axon to convey a signal to other neurons (Kandel et al. 1991).

\footnotetext{
${ }^{5}$ The release of vesicles is no genuinely random process, but from the viewpoint of electrophysiology the statement has much truth.
} 
A biologically realistic model of an entire neuron as sketched above easily requires hundreds of coupled, nonlinear differential equations (Segev and Burke 1998), whence - as Henry Tuckwell reminds the reader in his Introduction to theoretical neurobiology -

... [one] fundamental principle in neural modeling is that one should use the simplest model that is capable of predicting the experimental phenomena of interest. (Tuckwell 1988, p. 85)

In this spirit, detailed models for the generation of action potentials will not be discussed here, nor shall the complex geometry of the neuron be considered.

Neurons communicate with each other by sequences of stereotyped action potentials which they transmit via their axons. Since all these spikes are alike, information will only be carried by the timing of the spikes, but not by their particular shape. There is growing evidence that the fine temporal structure of these spike trains is essential to neural information processing (Bair and Koch 1996; deCharms and Merzenich 1996; Gabbiani et al. 1996; Strong et al. 1998). A particularly well studied phenomenon is the localization of sound sources in barn owls (Konishi 1991; Knudsen 1984): Auditory neurons in this specialist species respond to frequencies up to $8 \mathrm{kHz}$ with spikes that are precisely phase-locked to the stimulus. Spike trains from both ears are fed into coincidence detectors in the nucleus laminaris, which detect interaural time differences with an acuity of a few microseconds. This enables owls to measure the azimuthal location of a sound source, e.g. a mouse, to within $2^{\circ}$ (Gerstner et al. 1996). The investigation of such temporal coding requires neuron models able to predict the spike trains evoked by time-dependent stimuli.

Spiking neuron models are well suited to this purpose. They characterize the neuron by a single variable, the membrane potential, which evolves as the neuron is stimulated. An output spike is fired when some threshold condition is met, followed by a reset of the internal state of the neuron; see Gerstner (1999b) for a review. The archetypical spiking neuron model, the leaky integrate-andfire model of Lapicque (1907), will be introduced in Chapter 2. For the sake of brevity and precision, the term Ornstein-Uhlenbeck neuron has been coined for the leaky integrate-and-fire neuron driven by a deterministic stimulus and additive Gaussian white noise (Lánský and Rospars 1995). The results of this thesis are based on that model.

The theory of point processes is particularly suited to investigate the statistical structure of spike trains, i.e. sequences of spike times (Cox and Lewis 1966; Daley and Vere-Jones 1988). Its use in the neurosciences has been pioneered by Perkel, Gerstein, and Moore (1967). Neural responses are analyzed in the framework of that theory in this thesis. 


\section{Aims, scope, and organization}

This thesis will show that noise enhances the signal processing capabilities of neurons by means of stochastic resonance. Specifically, the coding of periodic stimuli into spike trains in the presence of white noise is investigated. Response properties are studied for both constrained stimulation, with a reset of the stimulus after every spike, and unconstrained "natural" stimulation. The study focuses on subthreshold stimuli, i.e. such that would not elicit spikes in the absence of noise. This choice is made because suprathreshold, periodic driving evokes complex $N: M$ phase-locking phenomena, and thus appears ill suited to transmit periodic stimuli (Keener et al. 1981; Tateno 1998). Spike trains fired in response to subthreshold stimulation, in contrast, preserve the periodicity of the stimulus. Furthermore, Kempter et al. (1998) have shown that neuronal coincidence detectors, which are central to stereophonic hearing, perform best for subthreshold stimulation.

The Ornstein-Uhlenbeck neuron is derived from Stein's more elementary model in Chapter 2. Numerical methods for the computation of interspikeinterval densities are developed, and some important properties of these densities proven. The canonical form of the stimuli used throughout the thesis is defined. Existing approximations to the Ornstein-Uhlenbeck neuron are evaluated in Chapter 3. The Arrhenius\&Current model is introduced as an improved approximation providing an explicit expression for the interspikeinterval density.

The response of the neuron to periodic stimuli without reset is analyzed by way of a Markov chain as proposed in Chapter 4. The elicited spike trains are comprehensively characterized in terms of the distribution of spikes with respect to the stimulus phase (spike phase density), the phase-averaged interspike-interval density, and their power spectral density. The spike intensity, well suited to visualize the temporal structure of responses, is derived from the Markov chain analysis as well. Corresponding results for constrained stimulation with reset are obtained from the theory of renewal processes.

Chapter 5 presents the results of this thesis. The preeminent finding is that the Ornstein-Uhlenbeck benefits from twofold stochastic resonance in response to unconstrained sinusoidal stimulation: The signal-to-noise ratio of the elicited spike train is maximized as a function of both input noise amplitude and stimulus frequency (Figure 5.14, p. 99). This effect is named stochastic double resonance (SDR) and prevails over a wide range of stimuli (Figure 5.17, p. 104). Moreover, the response properties of the neuron are shown to be either independent of or linearly related to stimulus properties under suitable scaling. The optimal scaled noise amplitude in particular is independent of all stimulus parameters and thus a universal property of the neuron (Figures 5.19 , p. 107 , and 5.25, p. 114). It is argued that the noise-induced resonance in stimulus frequency enables the neuron to operate as a bandpass filter in the 
following sense: Stimuli near the resonance frequency will evoke spike trains with clear temporal structure, while stimuli outside the preferred band will elicit temporally homogeneous trains. This filter may operate in a "transmission mode", with an optimal frequency independent of the stimulus amplitude, or in a "discrimination mode" with an amplitude-dependent optimal frequency (Figure 5.25, p. 114). In case of constrained stimulation with reset, stochastic resonance is demonstrated for peculiar stimuli (Figure 5.3, p. 86), but shown to vanish under biologically plausible conditions (Figure 5.6, p. 89). All findings are based on numerical investigations of the model and were largely verified by means of simulations. Their origins are discussed in detail. 


\section{Chapter 2}

\section{The Ornstein-Uhlenbeck Neuron}

This chapter introduces the Ornstein-Uhlenbeck neuron as the workhorse of this thesis. The model is derived from Stein's more elementary model, generalizing earlier results. It is shown that the stochastic input to Stein's model may be replaced by a deterministic stimulus with additive Gaussian white noise, provided that the original input comprised both excitatory and inhibitory components. A robust and efficient algorithm for the numerical computation of interspike-interval densities in response to arbitrary time-dependent stimuli is proposed, and it is proven that the latter densities are strictly positive. The canonical stimuli used throughout this thesis are defined towards the end of the chapter.

The Ornstein-Uhlenbeck neuron is defined as a leaky integrate-and-fire neuron exposed to additive Gaussian white noise. It is widely used to investigate the nature of the neural code (Maršálek et al. 1997; Troyer and Miller 1997; Bugmann et al. 1997; Feng 1997; Shadlen and Newsome 1998). The leaky integrate-and-fire neuron itself was first introduced by Lapicque (1907) in a discussion of membrane polarizability. It idealizes the neuron as a capacitor $C$, in parallel with an Ohmic resistance $R$ and a battery maintaining the potential $V_{L}$ to resemble ion channels and pumps, respectively, see Fig. 2.1. In the absence of input, the potential difference across the capacitor will be $v=V_{L}$. The exterior of the neuron is taken as reference potential, and $v$ is the potential of the interior of the neuron. It is referred to interchangeably as membrane potential, voltage, or polarization. The resting potential is $V_{L} \approx-65 \mathrm{mV}$ for typical neurons (Tuckwell 1988). The effective input current $i(t)$ may hyperpolarize $\left(v(t)<V_{L}\right)$ or depolarize $\left(v(t)>V_{L}\right)$ the membrane. Once the membrane is sufficiently depolarized for the potential $v(t)$ to reach a threshold $\Theta$, a spike is discharged. Immediately afterwards, the membrane potential is re-polarized to a reset value $v=V_{R}<\Theta$, which need not be identical to the resting value $V_{L}$. This yields the following equations for the dynamics of the 
(a)

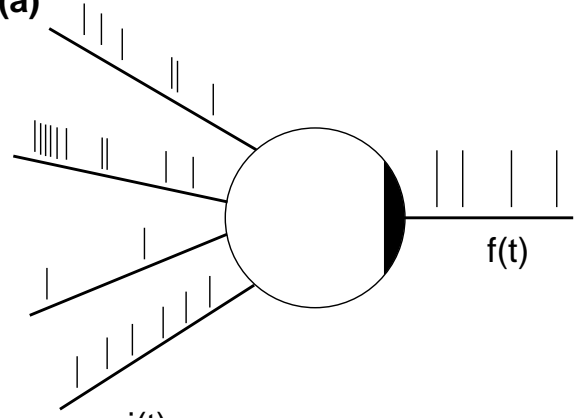

(b)

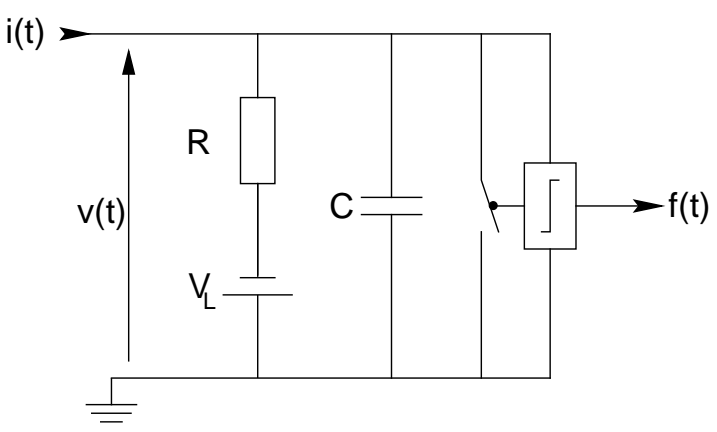

(c)

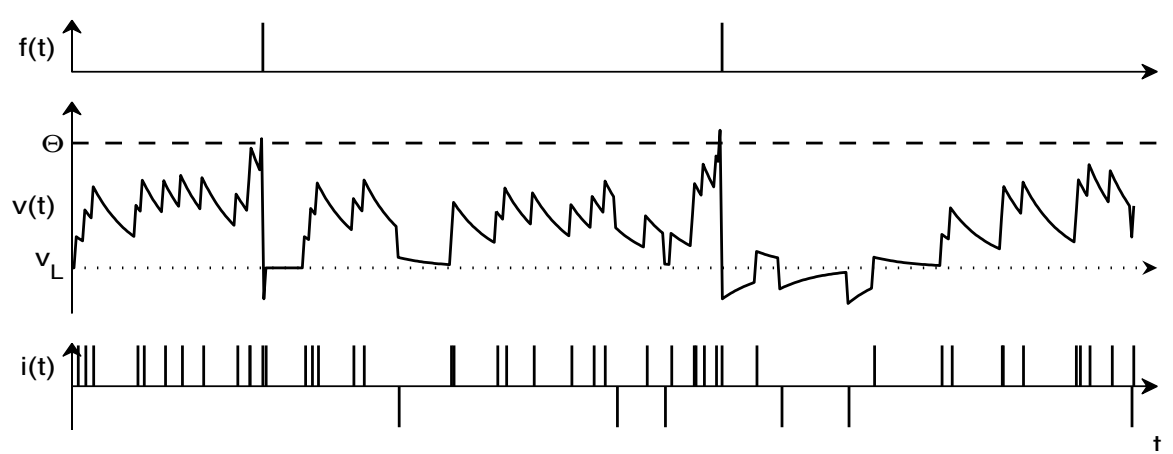

Figure 2.1: (a) Input $i(t)$ to the neuron arrives through the dendritic tree in form of small post-synaptic current pulses, which are added at the soma, and can initiate spikes at the axon hillock (black area). They propagate along the axon, and transmit the output $f(t)$ to other neurons. (b) The leaky integrate-and-fire neuron is a model of the spike generator at the axon hillock. The input current $i(t)$ charges the membrane capacitor $C$, while some current leaks through the membrane, i.e. resistor R. Ion pumps in the membrane try to maintain a potential $V_{L}$ in the absence of input. Once the potential $v(t)$ has reached a threshold, an output spike is initiated and the capacitor discharged. After Johnston and Wu (1995). (c) Evolution of membrane potential $v(t)$ (center) driven by excitatory and inhibitory pulse trains $i(t)$ (bottom). The output $f(t)$ of the neuron is shown at the top.

neuron,

$$
\tau_{m} \dot{v}(t)=-\left[v(t)-V_{L}\right]+R i(t), \quad v(t)=\Theta \Longrightarrow v\left(t^{+}\right)=v_{R} .
$$

Here, $\tau_{m}=R C$ is the effective membrane time constant of the neuron, and the dot denotes the derivative with respect to time. The output of the neuron is modeled as a sequence of $\delta$-spikes at the times $t_{j}$ of threshold crossings,

$$
f_{\mathbf{t}}(t)=\sum_{t_{j} \in \mathbf{t}} \delta\left(t-t_{j}\right), \quad \mathbf{t}=\left[t_{1}<t_{2}<t_{3} \ldots \mid v\left(t_{j}\right)=\Theta\right] .
$$

The ordered set $\mathbf{t}$ of spike times is called the spike train.

The major simplifying assumption of the model is to ignore the spatial complexity of the neuron. It merely describes the transformation of an effective 
input current $i(t)$, which results from dendritic processing of synaptic input, into an output spike train $f_{\mathbf{t}}(t)$ by the neuronal spike generator at the axon hillock. A further simplification is to replace the nonlinear dynamics of the spike generation process, as described by the Hodgkin-Huxley equations, by a linear model for the evolution of the membrane polarization, combined with a threshold condition for spike initiation. The spikes themselves are singular events superimposed onto the linear evolution.

Subtypes of the leaky integrate-and-fire model differ in the input currents they assume, a possibly time-dependent threshold function $\Theta(t)$, the reset potential $v_{R}$ or the presence of a lower bound on the hyperpolarization. The particular case of the Ornstein-Uhlenbeck neuron is discussed in the next section.

\subsection{Derivation from Stein's model}

Building on work by Gerstein and Mandelbrot (1964), Stein (1965) suggested to model the dendritic input current as a sum of Poisson processes,

$$
R i(t)=\sum_{k=1}^{K} \alpha_{k} \mathrm{~d} N_{\lambda_{k}}(t) .
$$

$N_{\lambda_{k}}(t)$ is a Poisson process with rate $\lambda_{k}$, so that $\mathrm{d} N_{\lambda_{k}}(t)$ is a series of $\delta$-pulses separated by exponentially distributed intervals. Positive pulse amplitudes $\alpha_{k}$ correspond to excitatory (depolarizing), negative amplitudes to inhibitory (hyperpolarizing) input. Inserting this input model into Eq. (2.1) yields Stein's neuron model

$$
\tau_{m} \dot{v}(t)=-\left[v(t)-V_{L}\right]+\sum_{k=1}^{K} \alpha_{k} \mathrm{~d} N_{\lambda_{k}}(t) .
$$

In this model, the evolution of the membrane potential is a Markov process with discrete jumps, i.e. its sample paths are discontinuous, ${ }^{1}$ complicating the analysis. Several authors have therefore suggested to approximate Stein's model by a continuous diffusion process in the limit of vanishing pulse amplitudes and diverging rates (Gluss 1967; Johannesma 1968). Rigorous results were first obtained by Kallianpur (1983) and Lánský (1984). The latter proved that both the sample paths and the interspike-interval density of Stein's model converge weakly to those of the Ornstein-Uhlenbeck process under certain conditions on the pulse amplitudes and rates. These results were obtained for time-independent rates and pulse amplitudes. They are extended to the most

\footnotetext{
${ }^{1}$ The sample paths are left-continuous.
} 
general case here, including time-dependent rates and amplitudes. The derivation follows the lines of earlier work (Capocelli and Ricciardi 1971; Matsuyama et al. 1974; Lánský 1984).

Inhomogeneity in time may arise through modulation of the pulse amplitudes $\alpha_{k}(t)$ as well as of the pulse rates $\lambda_{k}(t)$, both of which are assumed to be sufficiently smooth. Setting $\tau_{m}=1$ and $V_{L}=0$ for notational convenience yields the stochastic differential equation

$$
\dot{v}(t)=-v(t)+\sum_{k=1}^{K} \alpha_{k}(t) \mathrm{d} N_{\lambda_{k}(t)}(t) .
$$

The membrane potential $v$ is thus a random variable with transition probability

$$
\mathcal{P}(v, t+h \mid w, t)=\operatorname{Prob}\{\text { The potential is } v \text { at } t+h \text { if it was } w \text { at } t .\}
$$

To obtain the diffusion approximation to Stein's model, i.e. the OrnsteinUhlenbeck neuron model, a partial differential equation is constructed for the temporal evolution of $\mathcal{P}(v, t+h \mid w, t)$. This is then reduced to a Fokker-Planck equation via the Kramers-Moyal expansion (van Kampen 1992).

\subsubsection{Fokker-Planck equation}

Since the inputs $N_{\lambda_{k}(t)}(t)$ are inhomogeneous Poisson processes, there will be at most one input pulse within a sufficiently short input interval $h>0$. Thus, the potential can change within this interval in $K+1$ distinct ways:

- With probability $\left[1-h \sum_{k} \lambda_{k}(t)\right]+o(h)$, no input pulse arrives in $[t, t+h)$ and the potential merely decays exponentially: $v \longrightarrow v \mathrm{e}^{-h}$.

- With probability $h \lambda_{k}(t)+o(h)$, an input pulse from source $k \in\{1, \ldots, K\}$ arrives. In addition to the decay, the potential has a jump: $v \longrightarrow v \mathrm{e}^{-h}+\alpha_{k}(t+h)$.

In writing down these probabilities, $\int_{0}^{h} \lambda_{k}(t+s) \mathrm{d} s=h \lambda_{k}(t)+o(h)$ has been exploited. Further, the pulses are attached to the ends of the intervals to avoid discontinuities within. This yields for the transition probability density in the limit $h \rightarrow 0$

$$
\begin{aligned}
\mathcal{P}(v, t+h \mid w, t)=\left[1-h \sum_{k=1}^{K}\right. & \left.\lambda_{k}(t)\right] \delta\left(v-w \mathrm{e}^{-h}\right) \\
& +h \sum_{k=1}^{K} \lambda_{k}(t) \delta\left(v-\left[w \mathrm{e}^{-h}+\alpha_{k}(t+h)\right]\right),
\end{aligned}
$$

where $\delta(x)$ is Kronecker's delta function. 
The evolution of the membrane potential is a Markov process, since the neuronal capacitor is charged by uncorrelated Poissonian pulse sequences. Therefore, the Chapman-Kolmogorov equation holds and one has for arbitrary $v_{0}$ and $t \geq t_{0}(\operatorname{van}$ Kampen 1992)

$$
\begin{aligned}
\mathcal{P}\left(v, t+h \mid v_{0}, t_{0}\right)= & \int_{-\infty}^{\infty} \mathcal{P}(v, t+h \mid w, t) \mathcal{P}\left(w, t \mid v_{0}, t_{0}\right) \mathrm{d} w \\
= & {\left[1-h \sum_{k=1}^{K} \lambda_{k}(t)\right] \mathrm{e}^{h} \mathcal{P}\left(v \mathrm{e}^{h}, t \mid v_{0}, t_{0}\right) } \\
& +h \sum_{k=1}^{K} \lambda_{k}(t) \mathrm{e}^{h} \mathcal{P}\left(\left[v-\alpha_{k}(t+h)\right] \mathrm{e}^{h}, t \mid v_{0}, t_{0}\right) .
\end{aligned}
$$

The integral over $w$ is taken along the entire real axis, i.e. assuming that the membrane potential may take on any value, as is it the case when spike generation blocked pharmacologically. ${ }^{2}$ The derivation given here applies to spiking neurons nonetheless, because the distribution of the intervals between threshold crossings will be expressed in terms of the membrane potential distribution found in the absence of the threshold in Chapter 2.2. ${ }^{3}$ Taylor expansion of the Chapman-Kolmogorov equation (2.7) about $h=0$ yields

$$
\begin{aligned}
\mathcal{P}\left(v, t+h \mid v_{0}, t_{0}\right) & =\mathcal{P}\left(v, t \mid v_{0}, t_{0}\right)+h\left\{\frac{\partial}{\partial v}\left[v \mathcal{P}\left(v, t \mid v_{0}, t_{0}\right)\right]\right. \\
+ & \left.\sum_{k=1}^{K} \lambda_{k}(t)\left[\mathcal{P}\left(v-\alpha_{k}(t), t \mid v_{0}, t_{0}\right)-\mathcal{P}\left(v, t \mid v_{0}, t_{0}\right)\right]\right\}+O\left(h^{2}\right) .
\end{aligned}
$$

This immediately leads to

$$
\begin{aligned}
& \frac{\partial}{\partial t} \mathcal{P}\left(v, t \mid v_{0}, t_{0}\right)= \\
& \quad \frac{\partial}{\partial v}\left[v \mathcal{P}\left(v, t \mid v_{0}, t_{0}\right)\right]+\sum_{k=1}^{K} \lambda_{k}(t)\left[\mathcal{P}\left(v-\alpha_{k}(t), t \mid v_{0}, t_{0}\right)-\mathcal{P}\left(v, t \mid v_{0}, t_{0}\right)\right] .
\end{aligned}
$$

Since the jump amplitudes $\alpha_{k}(t)$ are assumed small, it is plausible to expand $\mathcal{P}\left(v-\alpha_{k}(t), t \mid v_{0}, t_{0}\right)$ about $v$ to obtain a differential equation for the transition probability. This Kramers-Moyal expansion yields (van Kampen 1992)

$$
\begin{aligned}
\frac{\partial}{\partial t} \mathcal{P}\left(v, t \mid v_{0}, t_{0}\right)=-\frac{\partial}{\partial v}\left[A_{1}(v, t)\right. & \left.\mathcal{P}\left(v, t \mid v_{0}, t_{0}\right)\right]+\frac{A_{2}(t)}{2} \frac{\partial^{2}}{\partial v^{2}} \mathcal{P}\left(v, t \mid v_{0}, t_{0}\right) \\
& +\sum_{n=3}^{\infty} \frac{(-1)^{n}}{n !} A_{n}(t) \frac{\partial^{n}}{\partial v^{n}} \mathcal{P}\left(v, t \mid v_{0}, t_{0}\right)
\end{aligned}
$$

\footnotetext{
${ }^{2}$ Application of tetrodotoxin (TTX) will serve this purpose (Johnston and Wu 1995).

${ }^{3}$ Previous publications on the diffusion approximation have altogether ignored this complication (Gluss 1967; Johannesma 1968; Capocelli and Ricciardi 1971).
} 
The coefficients $A_{n}(t)$ arising from the expansion are the infinitesimal or jump moments of the input process

$$
\begin{aligned}
A_{n}(v, t)=\lim _{h \rightarrow 0} \frac{1}{h} \int_{-\infty}^{\infty} z^{n} \mathcal{P}(v+z, t & +h \mid v, t) \mathrm{d} z \\
& = \begin{cases}-v+\sum_{k} \lambda_{k}(t) \alpha_{k}(t) & n=1 \\
\sum_{k} \lambda_{k}(t) \alpha_{k}^{n}(t) & n>1\end{cases}
\end{aligned}
$$

as is verified by inserting Eq. (2.6).

Equation (2.8) describes a continuous diffusion process if and only if all jump moments beyond $A_{2}$ vanish. Stein's neuron model can thus only be approximated by a diffusion process if all jump moments beyond $A_{2}$ vanish in the limit of infinitesimal jump amplitudes $\alpha_{k}(t)$ and diverging jump rates $\lambda_{k}(t)$. This requires the existence of sequences

$$
\lim _{j \rightarrow \infty} \alpha_{k}^{(j)}(t)=0, \quad \lim _{j \rightarrow \infty} \lambda_{k}^{(j)}(t)=\infty
$$

with

$$
\begin{aligned}
I(t)=v+\lim _{j \rightarrow \infty} A_{1}^{(j)}(v, t) & =\lim _{j \rightarrow \infty} \sum_{k=1}^{K} \lambda_{k}^{(j)}(t) \alpha_{k}^{(j)}(t)<\infty, \\
\frac{1}{2} \sigma^{2}(t)=\quad \lim _{j \rightarrow \infty} A_{2}^{(j)}(t) & =\lim _{j \rightarrow \infty} \sum_{k=1}^{K} \lambda_{k}^{(j)}(t)\left[\alpha_{k}^{(j)}(t)\right]^{2}<\infty, \\
\lim _{j \rightarrow \infty} A_{n}^{(j)}(t) & =\lim _{j \rightarrow \infty} \sum_{k=1}^{K} \lambda_{k}^{(j)}(t)\left[\alpha_{k}^{(j)}(t)\right]^{n}=0, n>2 .
\end{aligned}
$$

If these conditions are fulfilled, the following Fokker-Planck equation holds

$$
\frac{\partial}{\partial t} \mathcal{P}\left(v, t \mid v_{0}, t_{0}\right)=-\frac{\partial}{\partial v}\left[(-v+I(t)) \mathcal{P}\left(v, t \mid v_{0}, t_{0}\right)\right]+\frac{\sigma^{2}(t)}{2} \frac{\partial^{2}}{\partial v^{2}} \mathcal{P}\left(v, t \mid v_{0}, t_{0}\right)
$$

It corresponds to the Langevin equation

$$
\dot{v}(t)=-v(t)+I(t)+\sigma(t) \xi(t)
$$

for Gaussian white noise $\xi(t) . I(t)$ is thus identified as the deterministic stimulus and $\sigma(t)$ as the root mean square amplitude of the noise.

\subsubsection{Diffusion requires inhibition}

The membrane potential trajectories of Stein's model will converge to a diffusion process only if excitatory and inhibitory input are properly balanced. 
If only excitatory prevailed, the continuous sample path of a diffusion process would only be obtained if jump amplitudes tended to zero so fast that the noise amplitude would vanish, leaving an entirely deterministic process. To see this, define general sequences of pulse rates and amplitudes

$$
\lambda_{k}^{(j)}(t)=\sum_{m=1}^{M} j^{m} q_{k}^{(m)}(t), \quad \alpha_{k}^{(j)}(t)=\sum_{m=1}^{M} j^{-m} a_{k}^{(m)}(t)
$$

with smooth functions $q_{k}^{(m)}(t) \geq 0, a_{k}^{(m)}(t)>0$ independent of $j$. One then has from Eq. (2.11a)

$$
I(t)=\sum_{k=1}^{K} \sum_{m=1}^{M} q_{k}^{(m)}(t) a_{k}^{(m)}(t)+\lim _{j \rightarrow \infty} \sum_{k=1}^{K} \sum_{m>\ell}^{M} j^{m-\ell} q_{k}^{(m)}(t) a_{k}^{(\ell)}(t)
$$

which is finite only if

$$
q_{k}^{(m)}(t) a_{k}^{(\ell)}(t)=0 \text { for all } k \text { and } m>\ell \geq 1 .
$$

The noise amplitude then is from Eq. (2.11b)

$$
\begin{aligned}
\frac{\sigma^{2}(t)}{2}= & \sum_{k=1}^{K} \sum_{\ell, m}^{M}\left[q_{k}^{(m+\ell)}(t) a_{k}^{(\ell)}(t)\right] a_{k}^{(m)}(t) \\
& +\lim _{j \rightarrow \infty} \sum_{k=1}^{K} \sum_{m>i+\ell}^{M}\left[q_{k}^{(m)}(t) a_{k}^{(i)}(t)\right] a_{k}^{(\ell)}(t) j^{m-i-\ell}=0 .
\end{aligned}
$$

The last equality holds because the terms in brackets are zero from Eq. (2.14). Thus inhibition, i.e. $\alpha_{k}(t)<0$ for some $k$ is essential to the diffusion approximation.

A sequence of pulse amplitudes and rates converging to a diffusion process is given by Lánský (1997). To extend it to full generality, assume that the neuron receives $M$ pairs of inputs with balanced excitation and inhibition, and further $K$ unbalanced inputs. Choose the pulse rates and amplitudes for the balanced pairs as $(m=1, \ldots, M)$

$$
\begin{array}{ll}
\text { excitatory } & \kappa_{m}^{(j)}(t)=j^{2} q_{m}(t)>0, \quad \alpha_{m}^{(j)}(t)=\frac{a_{m}(t)}{j} \geq 0, \\
\text { inhibitory } & \nu_{m}^{(j)}(t)=j^{2} r_{m}(t)>0, \quad \beta_{m}^{(j)}(t)=-\frac{a_{m}(t) q_{m}(t)}{j r_{m}(t)} \geq 0 .
\end{array}
$$

The unbalanced inputs are chosen as $(k=1, \ldots, K)$

$$
\lambda_{k}^{(j)}(t)=j p_{k}(t) \geq 0, \quad \gamma_{k}^{(j)}(t)=\frac{c_{k}(t)}{j} .
$$


Inserting into Eq. (2.11) yields

$$
\begin{aligned}
I(t) & =\sum_{k=1}^{K} p_{k}(t) c_{k}(t) \\
\frac{\sigma^{2}(t)}{2} & =\sum_{m=1}^{M} \frac{q_{m}(t) r_{m}(t)+q_{m}^{2}(t)}{r_{m}(t)} a_{m}^{2}(t) \\
A_{n}(t) & =0, \quad n>2 .
\end{aligned}
$$

The net input current $I(t)$ arises from the unbalanced inputs, while the noise $\sigma^{2}(t)$ stems from the balanced inputs alone, in line with the results of the preceeding paragraph. An important consequence is that the input current and the noise are completely independent of each other; in particular, one may assume a combination of time-dependent input with stationary noise.

\subsubsection{Canonical form of the Ornstein-Uhlenbeck neuron}

Re-introducing the resting potential $V_{L}$ and the membrane time constant $\tau_{m}$ while taking the noise amplitude to be constant, yields the diffusion approximation to Stein's model equation (2.3) for time-dependent input

$$
\tau_{m} \dot{v}(t)=-\left[v(t)-V_{L}\right]+I(t)+\sigma \xi(t)
$$

This equation is the Langevin equation for the forced Ornstein-Uhlenbeck process (Uhlenbeck and Ornstein 1930). $\xi(t)$ is Gaussian white noise. ${ }^{4}$ The Ornstein-Uhlenbeck neuron model (OUN) is completed by the addition of a constant threshold for firing $\Theta$, and a lower bound for the membrane hyperpolarization $v \geq v_{\text {hyp }}$. To eliminate parameters redundant in the analysis, ${ }^{5}$ the membrane potential is measured relative to the resting potential $V_{L}$ and in units of the resting-to-threshold distance $\Theta-V_{L}$, while time is measured in units of the membrane time constant $\tau_{m}$,

$$
\bar{v}=\frac{v-V_{L}}{\Theta-V_{L}}, \quad \bar{t}=\frac{t}{\tau_{m}}, \quad \bar{I}(\bar{t})=\frac{I\left(\tau_{m} \bar{t}\right)}{\Theta-V_{L}}, \quad \bar{\sigma}=\frac{\sigma}{\sqrt{\tau_{m}}\left(\Theta-V_{L}\right)} .
$$

Thus follows the canonical form of the Ornstein-Uhlenbeck neuron model, where the bars have been dropped for clarity

$$
\begin{aligned}
\dot{v}(t) & =-v(t)+I(t)+\sigma \xi(t), \quad v(t) \geq v_{\text {hyp }}, \\
v(t) & =1 \Longrightarrow v\left(t^{+}\right)=v_{R}=v(t=0), \\
f_{\mathbf{t}}(t) & =\sum_{t_{j} \in \mathbf{t}} \delta\left(t-t_{j}\right), \quad \mathbf{t}=\left[t_{1}<t_{2}<t_{3} \ldots \mid v\left(t_{j}\right)=1\right], \\
\langle\xi(t)\rangle & =0, \quad\left\langle\xi(t) \xi\left(t^{\prime}\right)\right\rangle=\delta\left(t-t^{\prime}\right), \quad \xi(t) \text { Gaussian } .
\end{aligned}
$$

\footnotetext{
${ }^{4}$ The noise amplitude $\sigma$ is measured in units of $\mathrm{V} \sqrt{\mathrm{s}}$ in this definition.

${ }^{5}$ The scaling for the noise given above Eq. (2) of Plesser and Tanaka (1997) is wrong.
} 
$f_{\mathbf{t}}(t)$ is the output of the neuron firing the spike train $\mathbf{t}$. With the exception of Chapter 3, all results of this work are based on this Ornstein-Uhlenbeck neuron, and $v_{\text {hyp }}=-\infty$ will be assumed throughout. A few comments on the model are appropriate:

- Many neurons in the afferent sensory pathways receive far more descending input from cortical regions than ascending input from receptor cells, and several studies suggest that excitatory and inhibitory input are nearly balanced (Shadlen and Newsome 1998; van Vreeswijk and Sompolinsky 1996). These are precisely the conditions required for the diffusion approximation.

- The Ornstein-Uhlenbeck neuron reproduces responses of sensory neurons well, see Chapter 5.2.

- The form of the Langevin equation (2.20a) is independent of whether the time dependence arises from varying input rates or pulse amplitudes; see Lánský (1997).

- The Ornstein-Uhlenbeck neuron is a mathematically convenient approximation to the model of Stein. If the assumption of tiny pulses arriving in large numbers is violated, the behavior of the Ornstein-Uhlenbeck neuron may deviate considerably from Stein's model; Tuckwell and Cope (1980), but see Lánský (1984).

- The inclusion of reversal potentials for the input currents would introduce multiplicative noise to the diffusion approximation (Lánský and Lánská 1987) and greatly complicate the analysis. The main effect of the reversal potentials is to impose unattainable upper and lower boundaries for the membrane potential. If the upper boundary is below the threshold, the neuron would be silent, while a boundary above threshold is not felt. The upper boundary may therefore safely be ignored, while the lower boundary is mimicked by the reflecting lower boundary $v_{\text {hyp }}$.

\section{$2.2 \quad$ Interspike-interval density}

Neurons communicate with each other by the transmission of spikes, whence investigations of the neural code focus on the statistical properties of spike trains. These may be inferred from the distribution of interspike intervals as will be discussed in Chapter 4 . The problem is thus posed to determine at which time $t$ a neuron will fire the next spike if it has just fired at time $\hat{t}$. Since the neuron will spike once the membrane potential $v(t)$ reaches the threshold, this is a first-passage time problem. For the model neuron defined above, it is precisely the first-passage time problem of the Ornstein-Uhlenbeck 
process with an absorbing boundary at the threshold $\Theta=1$ and a reflecting boundary at $v_{\text {hyp }}$ (van Kampen 1992). No solutions to this problem are known for general time dependent stimuli, whence numerical methods are required. ${ }^{6}$

A robust and efficient numerical solution to this problem is developed here. It is based on the observation that the interspike-interval density may be expressed in terms of the membrane potential density one would find in the absence of a threshold (Schrödinger 1915). The resulting integral-equation representation is used to prove that the ISI density is strictly positive everywhere except at the origin, where it vanishes. This result, which is crucial to the analysis of Chapter 4.4, had only been conjectured hitherto (Tateno et al. 1995).

\subsubsection{Elementary properties}

Let $\hat{t}$ be the time of the most recent spike, so that $v\left(\hat{t}^{+}\right)=v_{R}$. The next spike will then be fired at $\hat{t}+\tau$, where the interspike interval $\tau$ (ISI) is defined by

$$
\tau=\inf \left\{s>0 \mid v(\hat{t}+s) \geq 1, v\left(\hat{t}^{+}\right)=v_{R}\right\} .
$$

In the presence of noise, the membrane potential is a random variable, and in consequence, the intervals $\tau$ as well. For time-dependent stimuli, the neuron will receive different input $I(\hat{t}+s)$ during intervals beginning at different times $\hat{t}$. Therefore, the distribution of the random variable $\tau$ is conditional on the time $\hat{t}$ of the beginning of the interval. The spike output of the neuron is thus characterized by the set of all conditional interspike-interval densities (cISID)

$$
\rho(\tau \mid \hat{t})=\operatorname{Prob}\{\text { The interval beginning at } \hat{t} \text { has length } \tau\} .
$$

The dependence of the ISI density on the stimulus $I(t)$ and the reset potential $v_{R}$ are implied in the notation. In the theory of stochastic processes, the interspike-interval density is known as first-passage-time density (FPTD).

The ISI density is more readily discussed in the framework of a boundary value problem of the Fokker-Planck equation derived in the previous section. With the change of variables given by Eq. (2.19) and assuming timeindependent noise, Eq. (2.12) becomes

$$
\frac{\partial}{\partial t} \mathcal{P}(v, t \mid w, s)=-\frac{\partial}{\partial v}[-v+I(t)] \mathcal{P}(v, t \mid w, s)+\frac{\sigma^{2}}{2} \frac{\partial^{2}}{\partial v^{2}} \mathcal{P}(v, t \mid w, s)
$$

For the interval beginning at $\hat{t}$, the initial condition is

$$
\mathcal{P}\left(v, t \mid v_{R}, \hat{t}\right)=\delta\left(v-v_{R}\right)
$$

\footnotetext{
${ }^{6}$ The graphical method of Scharstein (1979) applies only to the noise-free case $(\sigma=0)$. It cannot be adapted to the noisy model. See also Tuckwell (1988, Ch. 3.7).
} 
The threshold at $v=1$ is an absorbing boundary, and the limit of hyperpolarization a reflecting one, requiring

$$
\mathcal{P}\left(1, t \mid v_{R}, \hat{t}\right)=0,\left.\quad \frac{\partial}{\partial v} \mathcal{P}\left(v, t \mid v_{R}, \hat{t}\right)\right|_{v=v_{\mathrm{hyp}}}=0 .
$$

If the membrane potential is not bounded from below, i.e. $v_{\text {hyp }}=-\infty$, the reflecting boundary condition is equivalent to $\mathcal{P}\left(-\infty, t \mid v_{R}, \hat{t}\right)=0$.

The probability that the potential is below threshold at any $t>\hat{t}$ is

$$
G(t \mid \hat{t})=\int_{v_{\text {hyp }}}^{1} \mathcal{P}\left(v, t \mid v_{R}, \hat{t}\right) \mathrm{d} v
$$

and its time-derivative yields the conditional ISI density

$$
\rho(t-\hat{t} \mid \hat{t})=-\frac{\mathrm{d}}{\mathrm{d} t} \int_{v_{\text {hyp }}}^{1} \mathcal{P}\left(v, t \mid v_{R}, \hat{t}\right) \mathrm{d} v .
$$

If the third derivative of the stimulus $I(t)$ exists and is continuous, Theorems 3.13.3 and 3.14.1 of Gihman and Skorohod (1972) guarantee that the transition probability $\mathcal{P}(v, t \mid w, s)$ exists and is continuously differentiable at least once with respect to $t$ and $s$ and twice with respect to $v$ and $w$. Therefore, the interspike-interval density $\rho(\tau \mid \hat{t})$ exists and is continuous. The initial value of the conditional ISI density is $\rho(0 \mid \hat{t})=0$ if $v_{R}<1-\varepsilon, \varepsilon>0$, since the continuity of the potential trajectories precludes instantaneous jumps to the threshold. This condition on the reset potential $v_{R}$ simply requires a finite reset after each spike, which is a plausible assumption. $G(t \mid \hat{t})$ is the probability for the potential to be inside an interval which contains no sources of probability and has one absorbing boundary. Thus, it is a non-increasing function of $t$, and $\rho(\tau \mid \hat{t})$ is non-negative. From Eqs. (2.24) and (2.23b) follows furthermore that $\rho(\tau \mid \hat{t})$ is normalized

$$
\int_{\hat{t}}^{\infty} \rho(t-\hat{t} \mid \hat{t}) \mathrm{d} t=1-\lim _{t \rightarrow \infty} \int_{v_{\text {hyp }}}^{1} \mathcal{P}\left(v, t \mid v_{R}, \hat{t}\right) \mathrm{d} v=1 .
$$

The conditional interspike-interval density $\rho(\tau \mid \hat{t})$ is thus a well-defined, continuous probability density.

The first-passage time problem of the Ornstein-Uhlenbeck process was apparently first attacked by Siegert (1951) for constant input $I(t)=\mu$ using Laplace transforms. He derived a hierarchy of equations for the moments $M_{n}\left(v_{R}\right)$ of the first-passage-time density (ISI density) from the reset potential $v_{R}$ to threshold 1 . This has been extended to include the effects of a reflecting boundary at finite $v_{\text {hyp }}$ by Johannesma (1968). The resulting moment 
equations are

$$
M_{n}\left(v_{R}\right)=2 n \int_{\frac{v_{R}-\mu}{\sigma}}^{\frac{1-\mu}{\sigma}} \mathrm{d} w \mathrm{e}^{w^{2}} \int_{\frac{v_{\mathrm{hyp}}-\mu}{\sigma}}^{w} \mathrm{~d} z \mathrm{e}^{-z^{2}} M_{n-1}(z), \quad M_{0}\left(v_{R}\right)=1 .
$$

In particular, the mean interval length (mean first-passage time, MFPT) is

$$
\langle\tau\rangle=M_{1}\left(v_{R}\right)=\sqrt{\pi} \int_{\frac{v_{R}-\mu}{\sigma}}^{\frac{1-\mu}{\sigma}} \mathrm{d} w \mathrm{e}^{w^{2}}\left[\operatorname{erf} w-\operatorname{erf}\left(\frac{v_{\mathrm{hyp}}-\mu}{\sigma}\right)\right],
$$

where erf $x$ is the error function. All further moments are too complicated to be of much practical use (Stemmler 1996; Inoue et al. 1997). A closedform solution for the ISI density $\rho(\tau \mid \hat{t})$ is known only for the special case $I(t-\hat{t})=1+2 c \mathrm{e}^{(t-\hat{t})}$, cf. Chapter 3.2.

The second term in the square brackets in Eq. (2.28) is close to -1 even for $v_{\text {hyp }}=0$, unless the stimulus is weak or hyperpolarizing $(\mu \ll 1)$ or the noise very strong $(\sigma \gg \mu)$, since erf $x \rightarrow-1$ quickly for $x<0$. This indicates that the boundary of hyperpolarization may safely be set to $v_{\text {hyp }}=-\infty$ provided the neuron receives depolarizing input. This is confirmed by comparison to experimental data in Chapter 5.2.

\subsubsection{Schrödinger's renewal Ansatz}

A direct numerical evaluation of the ISI density becomes possible if one makes use of an idea due to Schrödinger (1915); see also Plesser and Tanaka (1997), Siegert (1951), and Gluss (1967). The only restriction of this method is that it cannot incorporate a reflecting lower boundary, i.e. $v_{\text {hyp }}=-\infty$ is required.

Schrödinger's approach sets out from the free diffusion solution $\mathcal{P}_{f}(v, t \mid w, s)$ of the Fokker-Planck equation (2.23a) for boundary and initial conditions

$$
\lim _{v \rightarrow \pm \infty} \mathcal{P}_{f}(v, t \mid w, s)=0, \quad \mathcal{P}_{f}(v, t=s \mid w, s)=\delta(v-w)
$$

As is obvious from the Langevin formulation of the problem (Eq. 2.20a), the membrane potential $v(t)$ is a linear transform of the input $I(t)$ with superimposed Gaussian white noise, and thus will have a Gaussian distribution itself. Indeed one has (Jung 1993)

$$
\mathcal{P}_{f}(v, t \mid w, s)=\frac{1}{\sqrt{2 \pi \eta^{2}(t-s)}} \exp \left\{-\frac{\left[v-v_{0}(t ; w, s)\right]^{2}}{2 \eta^{2}(t-s)}\right\},
$$

where

$$
\begin{aligned}
& v_{0}(t ; w, s)=\mathrm{e}^{-(t-s)}\left[w+\int_{0}^{t-s} I(s+u) e^{u} \mathrm{~d} u\right], \\
& \eta^{2}(t-s)=\frac{\sigma^{2}}{2}\left[1-\mathrm{e}^{-2(t-s)}\right],
\end{aligned}
$$




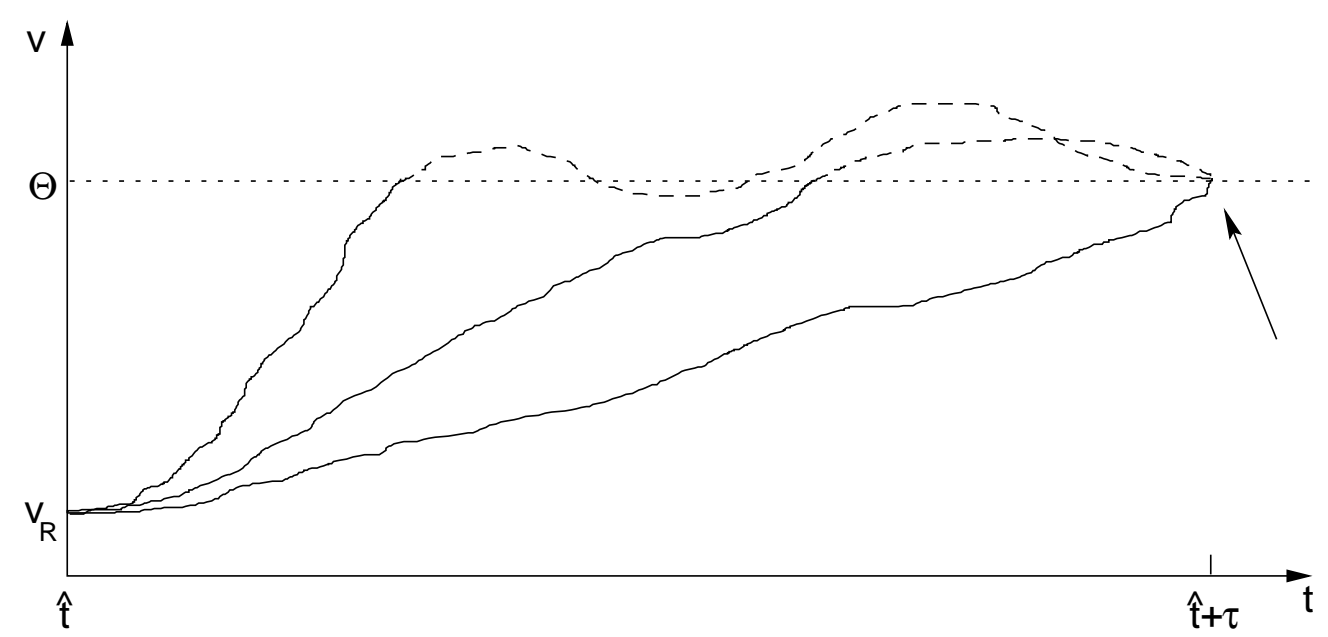

Figure 2.2: If the threshold is de-activated - e.g. by application of tetrodotoxin - the membrane potential evolves freely. The probability $\mathcal{P}_{f}\left(\Theta, \hat{t}+\tau \mid v_{R}, \hat{t}\right)$ to find the potential at the threshold $\Theta$ at time $\hat{t}+\tau$ (arrow) is given by integrating over all paths connecting $v\left(\hat{t}^{+}\right)=v_{R}$ with $v(\hat{t}+\tau)=\Theta$. Splitting each path into the first approach to threshold (solid part of lines) and later return to threshold (dashed part) yields Eq. (2.33).

are the membrane potential in the noise-free case and the variance of the potential, respectively. Schrödinger's idea was to split the trajectory of the freely evolving membrane potential from its starting value $v_{R}$ at $\hat{t}$ to the threshold $\Theta=1$ at $\hat{t}+\tau$ into two sections: the first approach to the threshold at $\hat{t}+u \leq \hat{t}+\tau$, and later returns to the threshold. This is illustrated in Fig. 2.2. Integrating over all first arrival times $\hat{t}+u$ yields

$$
\mathcal{P}_{f}\left(1, \hat{t}+\tau \mid v_{R}, \hat{t}\right)=\int_{0}^{\tau} \mathcal{P}_{f}(1, \hat{t}+\tau \mid 1, \hat{t}+u) \rho(u \mid \hat{t}) \mathrm{d} u
$$

The first-passage-time density $\rho(u \mid \hat{t})$ to the threshold is thus related to the free diffusion solution of the Fokker-Planck equation through an integral equation. Indeed, Eq. (2.33) is a special form of a renewal equation (van Kampen 1992). Note that $v_{0}(t ; w, s)$ depends on the absolute time $s$ through $I(s+u)$, whence $\mathcal{P}_{f}(v, t \mid w, s)$ cannot be rewritten as a function of $t-s$ alone. This precludes the use Laplace transforms to solve this renewal equation for time-dependent stimuli. It is this connection between the free potential distribution and the ISI density which justified the use of the Chapman-Kolmogorov equation (2.7) in Section 2.1.

The kernel of the renewal equation (2.33) has a square-root singularity at $\tau=u$. Defining

$$
\begin{aligned}
K_{\hat{t}}(\tau, u) & =\sqrt{\tau-u} \mathcal{P}_{f}(1, \hat{t}+\tau \mid 1, \hat{t}+u) \\
g_{\hat{t}}(\tau) & =\mathcal{P}_{f}\left(1, \hat{t}+\tau \mid v_{R}, \hat{t}\right)
\end{aligned}
$$


one obtains a generalized Abel equation (Linz 1985)

$$
g_{\hat{t}}(\tau)=\int_{0}^{\tau} \frac{K_{\hat{t}}(\tau, u)}{\sqrt{\tau-u}} \rho(u \mid \hat{t}) \mathrm{d} u .
$$

The kernel $K_{\hat{t}}(\tau, u)$ is regular with

$$
K_{\hat{t}}(\tau, \tau) \equiv \lim _{u \rightarrow \tau-} K_{\hat{t}}(\tau, u)=\frac{1}{\sqrt{2 \pi \sigma^{2}}} .
$$

Now both the left-hand side and the kernel of the renewal equation are values of time-independent Gaussians. Therefore, both $K_{\hat{t}}(\tau, u)$ and $g_{\hat{t}}(\tau)$ are differentiable everywhere. From Eqs. (2.34) and (2.37) follows that $K_{\hat{t}}(\tau, u)$ is strictly positive for all $u \leq \tau$. The same holds for $g_{\hat{t}}(\tau)$ for $\tau>0$ from Eq. (2.35), while $g_{\hat{t}}(0)=0$ from the initial conditions, Eq. (2.23b).

The Abel equation (2.36) lends itself to direct numerical evaluation for the interspike-interval density $\rho(u \mid \hat{t})$. The algorithm is given in Appendix A. It requires $\sim O\left(N^{2}\right)$ evaluations of the kernel $K_{\hat{t}}$ for the computation of the ISI density on $N$ points, while the numerical error drops $\sim N^{-2.9}$. The results reported in this work have been obtained with this algorithm.

The transformation of Schrödinger's renewal equation (2.33) to an Abel equation is not the only possibility to handle the kernel singularity. Plesser and Tanaka (1997) suggested to integrate out the singularity, but this can slow down computations seriously for small noise amplitudes. Buonocore et al. (1987) have proposed an algorithm based on a different integral equation for the ISI density. Their approach requires $\sim O\left(N^{2}\right)$ kernel evaluations as well, but appears to be less stable than the one given here.

\subsubsection{Strict positivity of the ISI density}

With the aid of the renewal equation (2.33), it can be proven that the ISI is strictly positive except at the origin, i.e.

$$
\rho(u \mid \hat{t})>0 \text { for all } u>0 .
$$

Intuitively, this is clear: Following the reset of the potential to $v_{R}<1-\varepsilon$, noise may induce (or prevent) threshold crossings at any time, albeit with astronomically small probability for some times. Since this property will be central to the analysis of Chapter 4.4, a rigorous proof by contradiction is given here. Let $\hat{t}=0$ and write $\rho(\tau) \equiv \rho(\tau \mid \hat{t})$ for the sake of brevity. As a first step, it is shown that $\rho(\tau)$ cannot be zero in any interval beginning at $\tau=0$. The same is then proven for any interval of finite length.

Assume that $a>0$ exists such that $\rho(\tau)=0$ for $0 \leq \tau \leq a$. Then from Eq. (2.36)

$$
g_{\hat{t}}(a)=\int_{0}^{a} K_{\hat{t}}(a, u) \rho(u) \mathrm{d} u=0 .
$$


which is a contradiction since $g_{\hat{t}}(\tau)>0$ for all $\tau>0$. Thus an $a>0$ exists such that $\rho(\tau)>0$ for all $\tau \in(0, a]$. Assume now that $\rho(\tau)=0$ for $0<b \leq \tau \leq c$. Then if follows that for all $t \in[b, c]$

$$
g_{\hat{t}}(t)=\int_{0}^{t} K_{\hat{t}}(t, u) \rho(u) \mathrm{d} u=\int_{0}^{b} K_{\hat{t}}(t, u) \rho(u) \mathrm{d} u .
$$

Since $g_{\hat{t}}(t)>0$ in $[b, c]$, division by $g_{\hat{t}}(t)$ and differentiation with respect to $t$ yields

$$
\int_{0}^{b}\left[\frac{\partial}{\partial t} \frac{K_{\hat{t}}(t, u)}{g_{\hat{t}}(t)}\right] \rho(u) \mathrm{d} u=0 \quad \text { for all } \quad t \in[b, c] .
$$

From the first part of the proof, an $a>0$ is known to exist with $(0, a] \subseteq(0, b]$ and $\rho(u)>0$ for all $u \in(0, a]$. Further, $\rho(u) \geq 0$ has been established for all $u \geq 0$ above, so that the integral vanishes only if the term in brackets is zero. Therefore one has for all $t \in(b, c]$ and $u \in(0, a]$

$$
\frac{\partial}{\partial t} \frac{K(t, u)}{g_{\hat{t}}(t)}=0 \quad \Longrightarrow \quad K_{\hat{t}}(t, u)=\alpha(u) g_{\hat{t}}(t) .
$$

This is a contradiction, since the kernel $K_{\hat{t}}(t, u)$ as defined by Eq. (2.34) cannot be split in this way over an interval of finite length, and the proof is completed.

\subsection{Stimuli}

The stimulus $I(t)$ driving the Ornstein-Uhlenbeck neuron is in principle entirely general. Since the existence and uniqueness of the interspike-interval density can strictly be established only if $I(t)$ has a continuous third derivative, it is convenient to limit the analysis to smooth stimuli. Stimuli composed of a constant base current $\mu$ and cosines of frequencies $\omega_{j}=j \Delta \omega$, i.e.

$$
I(t)=\mu+\frac{q}{\sqrt{\sum_{k} \alpha_{k}^{2}}} \sum_{j} \alpha_{j} \cos \left(\omega_{j} t+\phi_{j}\right)
$$

have all required properties and are sufficiently general. The noise-free membrane potential is then from Eq. (2.31)

$$
v_{0}(t+\tau ; w, t)=w \mathrm{e}^{-\tau}+\mu\left(1-\mathrm{e}^{-\tau}\right)+q\left[F(t+\tau)-\mathrm{e}^{-\tau} F(t)\right],
$$

with

$$
F(t)=\frac{1}{\sqrt{\sum_{k} \alpha_{k}^{2}}} \sum_{j} \frac{\alpha_{j}}{\sqrt{1+\omega_{j}^{2}}} \cos \left(\omega_{j} t+\phi_{j}-\operatorname{atan} \omega_{j}\right)
$$


Both $I(t)$ and $F(t)$ can efficiently be computed via discrete Fourier transforms. Independent of the particular choice of the amplitudes $\alpha_{j}$ and phases $\phi_{j}$, the mean square amplitude of the time-dependent stimulus component is

$$
\frac{q^{2}}{2}=\lim _{T \rightarrow \infty} \frac{1}{T} \int_{0}^{T}[I(t)-\mu]^{2} \mathrm{~d} t .
$$

For convenience, $q$, not $q / \sqrt{2}$, will be called the stimulus amplitude. The resulting excursions of the noise-free potentials have a mean square amplitude of

$$
\left\langle\Delta v_{0}^{2}\right\rangle=\lim _{T \rightarrow \infty} \int_{0}^{T}\left[v_{0}(\tau \mid \hat{t})-\mu\right]^{2} \mathrm{~d} \tau=\frac{q^{2}}{2 \sum_{k} \alpha_{k}^{2}} \sum_{j} \frac{\alpha_{j}^{2}}{1+\omega_{j}^{2}} .
$$

Aperiodic stimuli are characterized by a cut-off frequency $\Omega_{c}$ and are generated by drawing the phases $\phi_{j}$ at random from a uniform distribution over $[0,2 \pi)$, while the amplitudes are given by

$$
\alpha_{j}= \begin{cases}1 & 0<\omega_{j} \leq \Omega_{c} \\ \exp \left[-\frac{1}{2}\left(j-j_{c}\right)^{2}\right] & \Omega_{c}<\omega_{j}\end{cases}
$$

They will be used to evaluate the approximations to the Ornstein-Uhlenbeck neuron in Chapter 3.

Chapter 5 will focus on the processing of sinusoidal stimuli. They are obtained from the general stimulus of Eq. (2.39) by choosing $\alpha_{j}=\delta_{j k}$, where $\Omega=\omega_{k}$ is the stimulus frequency. One thus has

$$
I(t)=\mu+q \cos \left(\Omega t+\phi_{0}\right)
$$

and for the noise-free potential

$$
\begin{aligned}
& v_{0}(t+\tau ; w, t)=w \mathrm{e}^{-\tau}+\mu\left(1-\mathrm{e}^{-\tau}\right) \\
& \quad+\frac{q}{\sqrt{1+\Omega^{2}}}\left[\cos \left(\Omega(t+\tau)+\phi_{0}-\zeta\right)-\mathrm{e}^{-\tau} \cos \left(\Omega t+\phi_{0}-\zeta\right)\right]
\end{aligned}
$$

where $\zeta=\operatorname{atan} \Omega$ is the phase lag induced by the integration. The initial phase $\phi_{0}$ is taken to be zero unless stated otherwise. The modulation of the membrane potential is suppressed with increasing stimulus frequency due to the low-pass nature of the integrate-and-fire neuron. The stimulus parameters are visualized in Fig. 5.18, p. 106.

Stimuli are often classified as sub- or suprathreshold, depending on whether or not they are able to elicit spikes from a neuron in the absence of noise. To 
make this notion quantitative, each stimulus is characterized by its relative distance from threshold ${ }^{7}$

$$
\varepsilon=-\frac{1-\left(\mu+\sqrt{2\left\langle\Delta v_{0}^{2}\right\rangle}\right)}{\sigma}
$$

For periodic input, this definition yields

$$
\sigma \varepsilon=\left(\mu+\frac{q}{\sqrt{1+\Omega^{2}}}\right)-1=\sup _{\tau \geq 0} v_{0}(\tau \mid \hat{t})-1 .
$$

The relative distance is thus the minimum distance approached asymptotically once per stimulus period, relative to the root mean square amplitude of the noise.

Subthreshold stimuli have $\varepsilon<0$, suprathreshold stimuli $\varepsilon>0$. Note that for periodic stimuli $\varepsilon<0$ indeed guarantees that the stimulus is subthreshold, i.e. in the absence of noise the neuron never fires. For aperiodic input, however, the definition only holds in a root mean square sense, so that $v_{0}$ may occasionally cross the threshold even for $\varepsilon<0$.

\footnotetext{
${ }^{7}$ This definition here has the opposite sign as used in Plesser and Gerstner (1999).
} 



\section{Chapter 3}

\section{Approximating the Ornstein-Uhlenbeck Neuron}

Mathematically transparent approximations often provide far more insight into the nature of physical phenomena than precise solutions that are available only in implicit or numeric form. This holds for theoretical neurosciences as it does for physics. Several authors have therefore suggested approximations to the Ornstein-Uhlenbeck neuron. All of these "models of the model" propose compact expressions for the interspike-interval densities in response to time dependent stimuli (Abeles 1982; Gerstner and van Hemmen 1992; Gerstner 1995; Bulsara et al. 1996). This chapter provides the first rigorous evaluation of the various models abounding in the literature. A new approximation, the Arrhenius\&Current model, is introduced and is shown to be superior to all existing models. It is used in Chapter 5.3 to explain the parameter-dependence of stochastic resonance in the Ornstein-Uhlenbeck neuron.

The approximations tested in this chapter fall into two categories: hazard models (Plesser and Gerstner 1999) and the method-of-images approach of Bulsara et al. (1996). The models will be introduced below and are tested on a wide range of periodic and aperiodic inputs in Section 3.3.

\subsection{Hazard models}

In the absence of noise, the membrane potential $v_{0}\left(t ; v_{R}, \hat{t}\right)$ evolves according to Eq. (2.31) after a spike at $\hat{t}$. The next spike is fired when $v_{0}$ reaches the threshold. In the presence of noise, the neuron may fire even though the noise-free potential $v_{0}$ has not yet reached the threshold. An intuitive noise model can be based on the idea of an escape probability: At each moment of time, the neuron may fire with an instantaneous rate $h$ which depends on the momentary distance between the noise-free trajectory $v_{0}$ and the threshold $\Theta=1$, and possibly the momentary input current as well. More generally, one 
may introduce a hazard function

$$
h(\tau \mid \hat{t})=\operatorname{Prob}\left\{\begin{array}{l}
\text { After spike at } \hat{t}, \text { neuron fires at } \hat{t}+\tau, \\
\text { provided it has not fired in }[\hat{t}, \hat{t}+\tau) .
\end{array}\right\}
$$

The noise-free potential is written as $v_{0}(\tau \mid \hat{t}) \equiv v_{0}\left(\hat{t}+\tau ; v_{R}, \hat{t}\right)$ in this chapter for compactness. Once the hazard function known, the interspike-interval density is given by (Cox and Lewis 1966)

$$
\rho_{h}(\tau \mid \hat{t})=h(\tau \mid \hat{t}) \exp \left[-\int_{0}^{\tau} h(s \mid \hat{t}) \mathrm{d} s\right], \quad h(\tau \mid \hat{t}) \geq 0 .
$$

The exponential term accounts for the probability that a neuron "survives" from $\hat{t}$ to $\hat{t}+\tau$ without firing; the factor $h(\tau \mid \hat{t})$ gives the firing probability at $\hat{t}+\tau$, provided that the neuron has survived thus far. The cumulative distribution of the interspike intervals is thus

$$
P_{h}(\tau \mid \hat{t})=\int_{0}^{\tau} \rho_{h}(s \mid \hat{t})=\exp \left[-\int_{0}^{\tau} h(s \mid \hat{t}) \mathrm{d} s\right] \leq 1 .
$$

The interspike-interval density $\rho_{h}$ is positive by definition and is properly normalized, provided that $\int_{0}^{\tau} h(s \mid \hat{t}) \mathrm{d} s \rightarrow \infty$ as $\tau \rightarrow \infty$, which is a minor restriction. Thus $\rho_{h}$ is a proper probability density independent of the specific choice of the hazard. Note that $\rho_{h}(\tau \mid \hat{t})>0$ whenever $h(\tau \mid \hat{t})>0$. Finally, Eq. (3.2) may be inverted to express the hazard in terms of the ISI density

$$
h(\tau \mid \hat{t})=\frac{\rho_{h}(\tau \mid \hat{t})}{1-P_{h}(\tau \mid \hat{t})}=\frac{\rho(\tau \mid \hat{t})}{1-\int_{0}^{\tau} \rho(s \mid \hat{t}) \mathrm{d} s} .
$$

Five hazard models of neuronal dynamics are analyzed here. The various models differ in the choice of the hazard function $h(\tau \mid \hat{t})$ as shown in Fig. 3.1.

\subsubsection{Arrhenius model}

Assume that the membrane potential $v$ is - on average - not too close to the threshold. The influence of the threshold will then be small, and the potential be distributed roughly according to the density $\mathcal{P}_{f}\left(v, \hat{t}+\tau \mid v_{R}, \hat{t}\right)$ for the threshold-free case, see Eq. (2.30). Comparison of Eqs. (2.31) and (2.32) shows that the variance $\eta^{2}(\tau)$ of $\mathcal{P}_{f}$ approaches its asymptotic value $\sigma^{2} / 2$ twice as fast as $v_{0}(\tau \mid \hat{t})$ "forgets" initial conditions and past input. Thus, the density for the membrane potential may be approximated by

$$
\mathcal{P}_{h}\left(v, \tau \mid v_{R}, \hat{t}\right)=\frac{1}{\sqrt{\pi \sigma^{2}}} \exp \left\{-\frac{\left[v-v_{0}(\tau \mid \hat{t})\right]^{2}}{\sigma^{2}}\right\} .
$$



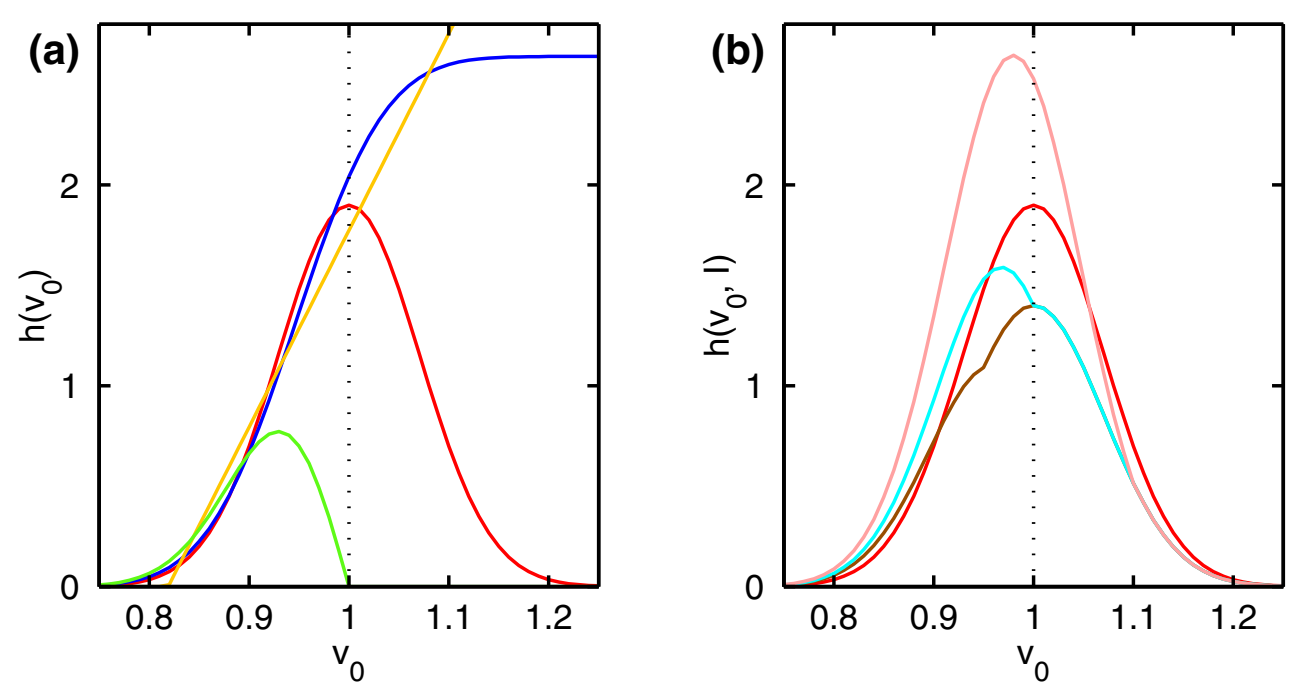

Figure 3.1: Hazard $h\left(v_{0}, I\right)$ as function of the noise free potential $v_{0}$ and input current $I$ [(b) only]. (a) Arrhenius (red), Abeles (blue), linear ramp (yellow) and Tuckwell models (green). (b) Arrhenius\&Current model for different values of the input current: $I=0$ (red), 0.95 (brown), 1 (cyan), and 1.1 (pink). The kinks in the hazard functions for this model result from the probability current term $[Y(\tau \mid \hat{t})]_{+}$. The vertical dotted line marks the threshold. The noise amplitude is $\sigma=0.1$.

The neuron fires a spike when the membrane potential reaches the threshold $v=1$. It is thus plausible to assume that the firing hazard is proportional to the probability that the potential is at the threshold (see Fig. 3.2),

$$
h_{\text {Arr }}(\tau \mid \hat{t})=w \mathrm{e}^{-x(\tau \mid \hat{t})^{2}} \sim \mathcal{P}_{h}\left(1, \tau \mid v_{R}, \hat{t}\right), \quad w^{\mathrm{opt}}=0.95 .
$$

Here,

$$
x(\tau \mid \hat{t})=\frac{1-v_{0}(\tau \mid \hat{t})}{\sigma}
$$

is the relative distance of the noise-free membrane potential from the threshold. The constant $w=w^{\text {opt }}=0.95$ was determined via an optimization procedure described in Section 3.3.1.

In physical terms, the membrane potential fluctuates about the noise-free potential $v_{0}$, and a spike is fired whenever this diffusion pushes the potential $v$ across the threshold, or barrier. The barrier height is $1-v_{0}$, corresponding to the activation energy $\left(1-v_{0}\right)^{2}$ required for a spike, while the fluctuations provide "thermal" energy $\sigma^{2}$. Statistical physics then predicts that the activation rate, i.e. the hazard, is given by an Arrhenius Ansatz as used above (van Kampen 1992).

For strongly suprathreshold stimuli the noise-free potential may become much larger than the threshold $\left(v_{0} \gg 1\right)$, implying an exponentially vanishing 


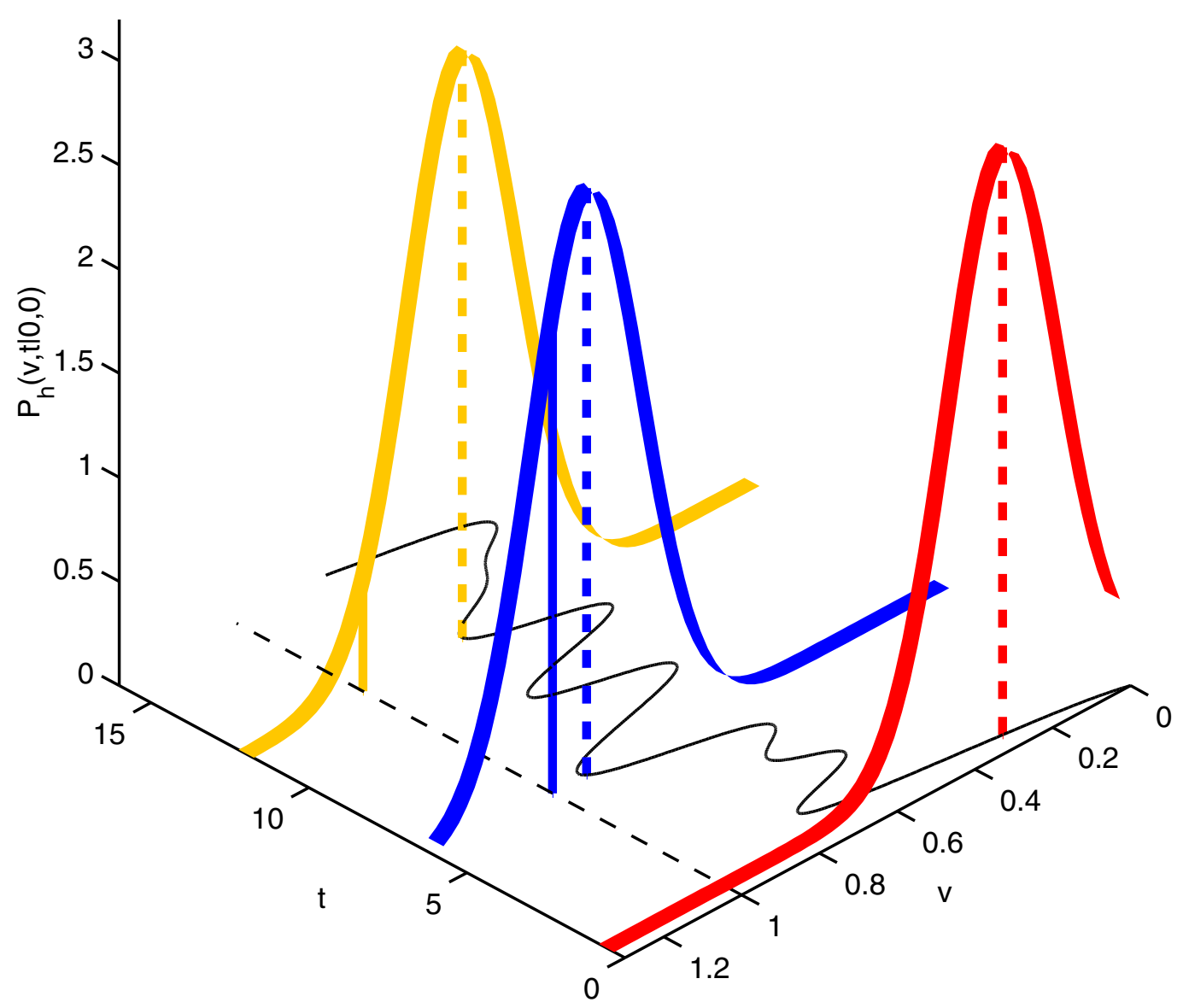

Figure 3.2: In the Arrhenius model, the hazard is proportional to the "free" probability density $\mathcal{P}_{h}$ at the threshold, as indicated by the solid bars under the Gaussians. The jagged black line is the noise-free potential $v_{0}(t)$, and the dashed black line the threshold. The true density of the potential vanishes for $v \geq 1$.

hazard, cf. Fig. 3.1(a). This might seem paradoxical at first, but is of little concern as long as the input $I(t)$ has no sharp transients. Then, $v_{0}(\tau \mid \hat{t})$ reaches the threshold only along continuous trajectories and suprathreshold levels of the potential are accessible only via periods of maximum hazard, so that the neuron will usually have fired before $v_{0}(\tau \mid \hat{t})$ becomes significantly suprathreshold.

\subsubsection{Arrhenius\&Current model}

Strong positive transients in the input current will push the membrane potential towards and possibly across the threshold in a time that is short on the timescale of diffusion. The potential density $\mathcal{P}_{h}$ is then shifted as a whole and, the argument given in the last paragraph of the preceeding section will no longer hold. In consequence, one expects that the simple Arrhenius Ansatz 


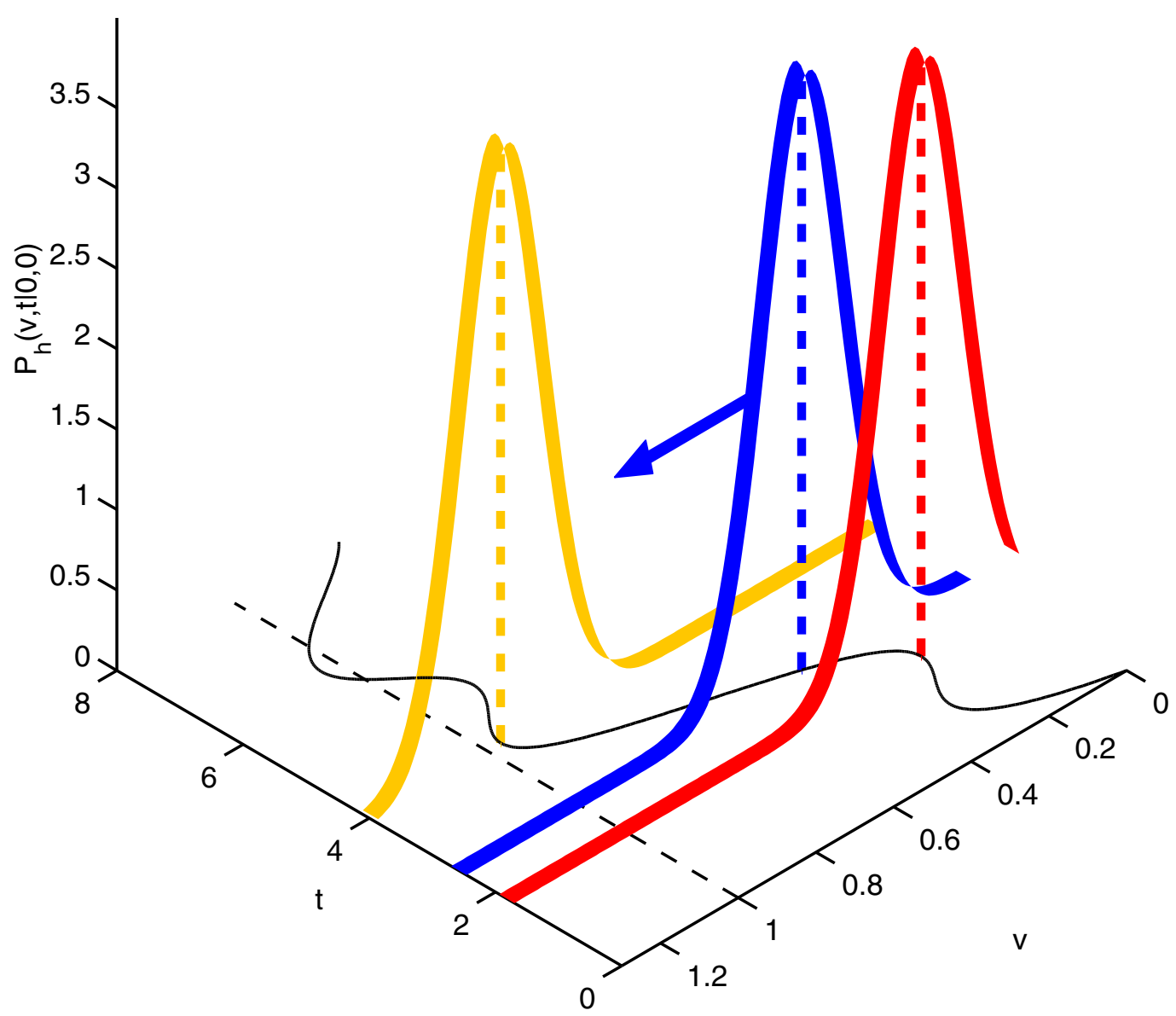

Figure 3.3: The Arrhenius\&Current model incorporates the probability current across the threshold caused by rapid shifts of the membrane potential density. This accommodates transients in the membrane potential $v_{0}(t)$ that are much faster than noise-induced diffusion across the threshold.

will not reproduce these transients well. This suggests to include in the hazard the probability current induced by shifting the probability density at the threshold, $\mathcal{P}_{h}(\Theta=1, \tau \mid \hat{t})$, across threshold with the speed of the center of this density, $\dot{v}_{0}(\tau \mid \hat{t})=-v_{0}(\tau \mid \hat{t})+I(\hat{t}+\tau)$, see Fig. 3.3. Since no spikes can be induced by drift from above threshold downwards, all negative currents are set to zero. The drift probability current therefore is

$$
J_{\text {drift }}(\tau \mid \hat{t})=\left[-v_{0}(\tau \mid \hat{t})+I(\hat{t}+\tau)\right]_{+} \mathcal{P}_{h}(\Theta=1, \tau \mid \hat{t})=\frac{[Y(\tau \mid \hat{t})]_{+}}{\sqrt{\pi}} \mathrm{e}^{-x(\tau \mid \hat{t})^{2}}
$$

with the relative probability current at threshold

$$
Y(\tau \mid \hat{t})=\frac{-v_{0}(\tau \mid \hat{t})+I(\hat{t}+\tau)}{\sigma}=\frac{1}{\sigma} \frac{\mathrm{d}}{\mathrm{d} \tau} v_{0}(\tau \mid \hat{t})
$$




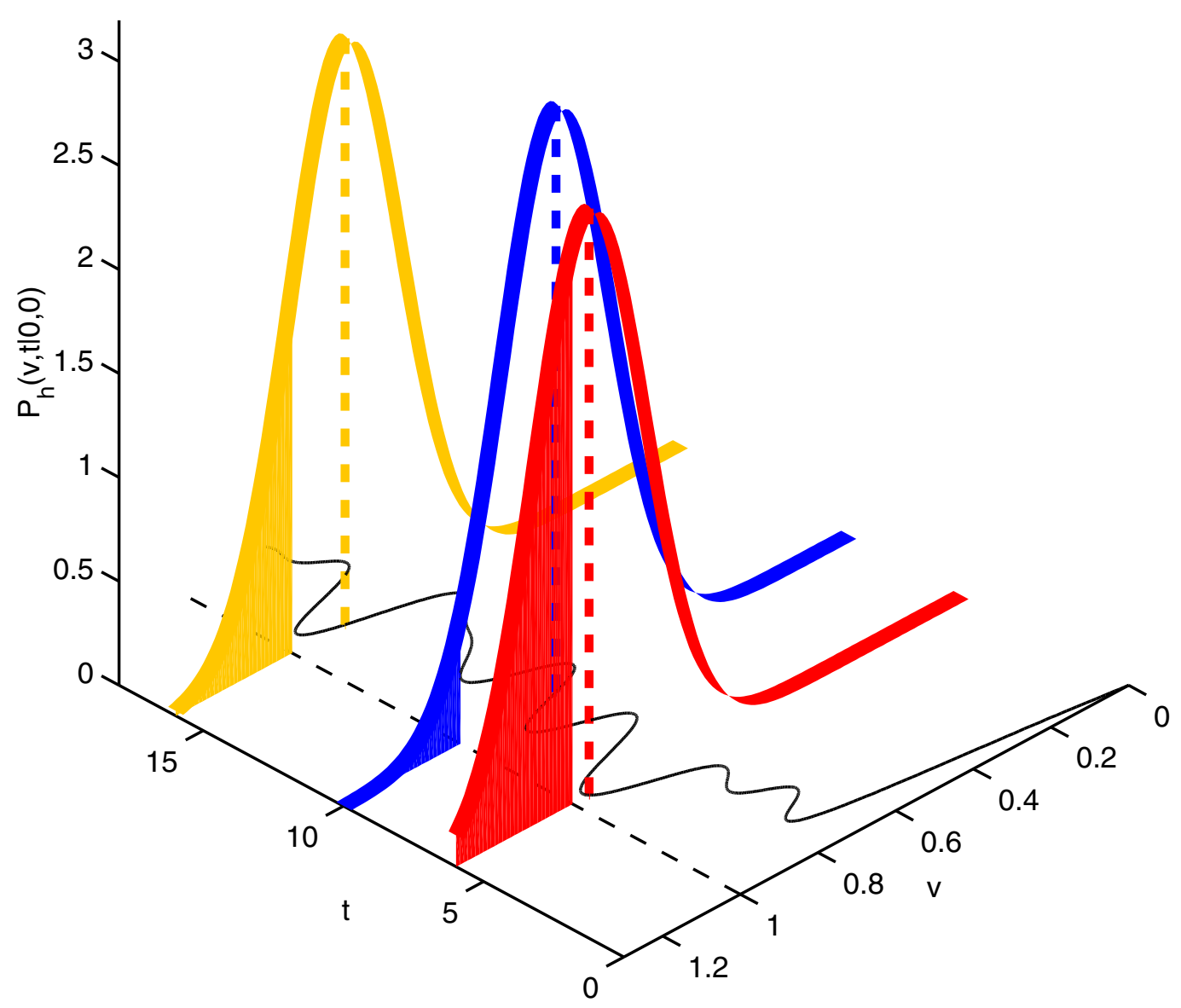

Figure 3.4: Abeles (1982) suggested to take the hazard proportional to the "free" probability of the membrane potential to be above the threshold, as indicated by the shaded areas under the Gaussians.

and $x(\tau \mid \hat{t})$ from Eq. (3.6); $[Y]_{+}=(Y+|Y|) / 2$. Together with the diffusive Arrhenius term the hazard function becomes

$$
h_{\mathrm{A} \& \mathrm{C}}(\tau \mid \hat{t})=\left(w_{1}+w_{2}[Y(\tau \mid \hat{t})]_{+}\right) \mathrm{e}^{-x(\tau \mid \hat{t})^{2}}, \quad w_{1}^{\mathrm{opt}}=0.70, \quad w_{2}^{\mathrm{opt}}=0.68 .
$$

As before, $w_{1}$ and $w_{2}$ are free parameters with optimal values $w_{1,2}^{\text {opt }}$, and $w$ is from the Arrhenius model. The first term of the hazard will be called diffusion and the second one drift term. The latter introduces kinks into the hazard function unless $\partial_{t} Y(t \mid \hat{t})=0$ wherever $Y(t \mid \hat{t})=0$, see Fig. 3.1(b).

\subsubsection{Abeles model}

Abeles (1982) suggested that the hazard should be related to the proportion of the membrane potential density $\mathcal{P}_{h}$ beyond the threshold, i.e. $h(\tau \mid \hat{t}) \sim$ $\int_{\Theta}^{\infty} \mathcal{P}_{h}(v, \tau \mid \hat{t}) \mathrm{d} v$, see Fig. 3.4. This Ansatz is somewhat questionable, as the 
correct potential density vanishes beyond the threshold. The widespread use of sigmoidal activation functions in the theory of neural networks (Wilson and Cowan 1972), nevertheless warrants a test of this model. To leave room for improvement, two free parameters $w_{1}$ and $w_{2}$ are included:

$$
h_{\mathrm{Ab}}(\tau \mid \hat{t})=w_{1} \operatorname{erfc}\left[x(\tau \mid \hat{t})-w_{2}\right], \quad w_{1}^{\mathrm{opt}}=0.66, w_{2}^{\mathrm{opt}}=0.53
$$

$\operatorname{erfc}(x)=1-\operatorname{erf}(x)$ is the complementary error function. Given the likeness of error function and hyperbolic tangent, the latter has not been investigated extensively; preliminary results indicated negligible differences.

\subsubsection{Linear ramp model}

Figure 3.1(a) suggests that both the Arrhenius and the Abeles hazards are approximately linear for subthreshold values of the membrane potential. Since a linear Ansatz greatly simplifies any subsequent analysis, it is well worth to test a linear ramp hazard function

$$
h_{\operatorname{lin}}(\tau \mid \hat{t})=w_{2}\left[w_{1}-x(\tau \mid \hat{t})\right]_{+}, \quad w_{1}^{\mathrm{opt}}=1.81, \quad w_{2}^{\mathrm{opt}}=0.49
$$

\subsubsection{Tuckwell model}

Suppose that the input $I(t)$ varies sufficiently slowly so that one may, at any point of time, take the membrane potential of the neuron to be stationary. A stationary potential $v_{0}(\tau \mid \hat{t})$ corresponds to a constant input current $I(\hat{t}+\tau)=$ $v_{0}(\tau \mid \hat{t})$. In this case, the average firing rate, can be approximated in closed form ${ }^{1}$ (Tuckwell 1989)

$$
\nu(\tau \mid \hat{t})=\frac{x(\tau \mid \hat{t})}{\sqrt{\pi}} \mathrm{e}^{-x(\tau \mid \hat{t})^{2}}
$$

For positive $\nu$, this may immediately be interpreted as hazard, and including a parameter for adjustment yields

$$
h_{\text {Tuck }}(\tau \mid \hat{t})=w \sqrt{\pi}[\nu(\tau \mid \hat{t})]_{+}=w[x(\tau \mid \hat{t})]_{+} \mathrm{e}^{-x(\tau \mid \hat{t})^{2}}, \quad w^{\text {opt }}=0.90
$$

The approximation for the firing rate is valid only for stimuli far below threshold, i.e. $x(\tau \mid \hat{t}) \gg 1$.

\footnotetext{
${ }^{1}$ This follows from Eq. (2.28) since $\langle\tau\rangle \approx 2 \int_{0}^{x} \mathrm{e}^{w^{2}} \mathrm{~d} w$ for $x=(1-\mu) / \sigma \gg 1$ by virtue of the asymptotic expansion of the error function $\operatorname{erf} z \sim 1-\frac{\mathrm{e}^{-z^{2}}}{z \sqrt{\pi}}\left[1+O\left(|z|^{-z}\right)\right]$ for $z \rightarrow \infty$; Gradsteyn and Ryzhik (1980).
} 


\subsection{Method-of-images approximation}

Bulsara et al. (1996) suggest to employ the method of images to obtain an approximation to the first-passage-time density of the Ornstein-Uhlenbeck process. With $\mathcal{P}_{f}(v, t \mid w, s)$ of Eq. (2.30) being the solution of the Fokker-Planck equation (2.23a) with natural boundaries, the function

$$
\mathcal{P}_{b}\left(v, \tau \mid v_{R}, \hat{t}\right)=\mathcal{P}_{f}\left(v, \hat{t}+\tau \mid v_{R}, \hat{t}\right)-\mathrm{e}^{\psi(\tau \mid \hat{t})} \mathcal{P}_{f}\left(v, \hat{t}+\tau \mid 2 \Theta-v_{R}, \hat{t}\right)
$$

with $^{2}$

$$
\psi(\tau \mid \hat{t})=\frac{2}{\eta^{2}(\tau)}\left(v_{R}-\Theta\right)\left[\Theta\left(1-\mathrm{e}^{-\tau}\right)-v_{0}(\tau \mid \hat{t})+v_{R} \mathrm{e}^{-\tau}\right] \mathrm{e}^{-\tau}
$$

will fulfill the boundary condition at the threshold $\Theta$, Eq. (2.23c), and the initial condition, Eq. (2.23b), for $v \leq \Theta . \eta^{2}(\tau)$ is the variance of $\mathcal{P}_{f}$, see Eq. (2.32). The threshold $\Theta=1$ is explicitly given here for clarity. Since $\mathcal{P}_{f}$ is a solution of the linear Fokker-Planck equation $(2.23 \mathrm{a}), \mathcal{P}_{b}$ will be a solution if and only if $\psi(\tau \mid \hat{t})$ is independent of $\tau$. This condition yields a differential equation for the noise-free potential ${ }^{3}$

$$
\dot{\psi}(\tau \mid \hat{t})=\dot{v}_{0}(\tau \mid \hat{t})\left(1-\mathrm{e}^{-2 \tau}\right)+\left[\Theta-v_{0}(\tau \mid \hat{t})\right]\left(1+\mathrm{e}^{-2 \tau}\right)+2\left(v_{R}-\Theta\right) \mathrm{e}^{-\tau}=0 .
$$

The dot denotes differentiation with respect to $\tau$. This equation yields

$$
v_{0}(\tau \mid \hat{t})=v_{R} \mathrm{e}^{-\tau}+\Theta\left(1-\mathrm{e}^{-\tau}\right)+2 c \sinh \tau
$$

requiring as input current

$$
I(\tau)=\Theta+2 c \mathrm{e}^{\tau}, \quad c \in \mathbb{R} .
$$

This input is rather peculiar: for $c \neq 0, \mathrm{Eq}$. (3.15) requires that the input be reset to $I(0)=\Theta$ after every spike - a rather implausible condition. But exponentially growing input is highly implausible anyways, leaving $c=0$, and thus $I(t)=\Theta$, as the only relevant solution. This is exactly the case solved by Sugiyama et al. (1970) using the method of images; it is used in Appendix A to test the algorithm proposed in Chapter 2.2. For the biologically interesting case of time-dependent input, $\mathcal{P}_{b}$ will at best be an approximate solution.

Inserting $\mathcal{P}_{b}\left(v, t \mid v_{R}, \hat{t}\right)$ into Eq. (2.25) yields the method-of-images approximation (MOI) to the first-passage-time density

$$
\begin{aligned}
\rho_{b}(\tau \mid \hat{t})=\frac{\sigma^{2}}{\sqrt{2 \pi} \eta^{3}(t)} & \left(\Theta-v_{R}\right) \mathrm{e}^{-\tau} \exp \left\{-\frac{\left[\Theta-v_{0}(\tau \mid \hat{t})\right]^{2}}{2 \eta^{2}(\tau)}\right\} \\
+ & \frac{\dot{\psi}(\tau \mid \hat{t})}{2} \mathrm{e}^{\psi(\tau \mid \hat{t})}\left[1+\operatorname{erf} \frac{\Theta-v_{0}\left(\tau \mid 2 \Theta-v_{R}, \hat{t}\right)}{\sqrt{2 \eta^{2}(\tau)}}\right]
\end{aligned}
$$

\footnotetext{
${ }^{2}$ The term $v_{R} \mathrm{e}^{-t}$ is missing in Eq. (11) of Bulsara et al. (1996).

${ }^{3}$ The corresponding Eq. (13) of Bulsara et al. (1996) is wrong.
} 
In contrast to the hazard models, neither the positivity nor the normalization of $\rho_{b}$ is assured.

\subsection{Evaluation}

The quality of the approximations defined above is assessed by comparing them to the Ornstein-Uhlenbeck neuron introduced in Chapter 2. Since the dynamics of this neuron model are fully characterized by the conditional interspike-interval density, the deviation of the ISI densities $\rho_{\text {mod }}$ of the models from the corresponding density $\rho$ for the OU neuron as given by Eq. (2.33) will be the foremost criterion. For the method-of-images approximation, the violation of positivity and normalization will be evaluated as well.

The deviation of the ISI densities is measured by the relative integrated mean square error (rIMSE)

$$
E=\frac{\int_{0}^{\infty} \mathrm{d} \tau\left[\rho(\tau \mid \hat{t})-\rho_{\text {mod }}(\tau \mid \hat{t})\right]^{2}}{\int_{0}^{\infty} \mathrm{d} \tau \rho(\tau \mid \hat{t})^{2}}
$$

The denominator ensures that $E$ is a scale-invariant error measure (Scott 1992). Figure 3.5 shows some examples and should give an intuitive idea of the error associated with various values of $E$. Other measures for the distance of probability densities, like the Kullback-Leibler divergence (Cover and Thomas 1991), were explored and lead to similar results.

The results presented here were obtained from a set of 14400 periodic and the same number of aperiodic stimuli as defined in Chapter 2.3. Base currents were in the range $0.55 \leq \mu \leq 1.2$, stimulus amplitudes approximately $0.14(1-$ $\mu)<q<2.1(1-\mu)$, and stimulus/cutoff frequencies $0.02 \pi \leq \Omega_{c} \leq 2 \pi$. For each set of these parameters, five phases were chosen randomly from $[0,2 \pi)$ for periodic stimuli, and five different random stimuli generated for the aperiodic case. The reset potential was $v_{R}=0$ throughout. Each stimulus was tested at eight noise amplitudes, randomly chosen to yield a uniform distribution of relative distances from threshold within $0.1<|\varepsilon|<3$. Approximately twothirds of all stimuli were subthreshold.

Some stimuli were excluded from the analysis. Rejection was based solely on the ISI densities computed for the diffusion approximation. Specifically, stimuli were excluded if the firing probability was so low that their norm was insufficient $\left(\int_{0}^{T} \rho(\tau) \mathrm{d} \tau<0.8\right)$, where $T$ was 20 stimulus periods for periodic and 409.4 for aperiodic stimuli. Furthermore stimuli were rejected if the numerical algorithm was unstable at the time resolution chosen. For some of these cases it was verified that - with appropriate discretization - the algorithm did converge. The instabilities were caused by very steep threshold-crossings of the membrane potential in combination with small noise. These instabilities did not affect subthreshold periodic stimuli. After defective stimuli were excluded, 

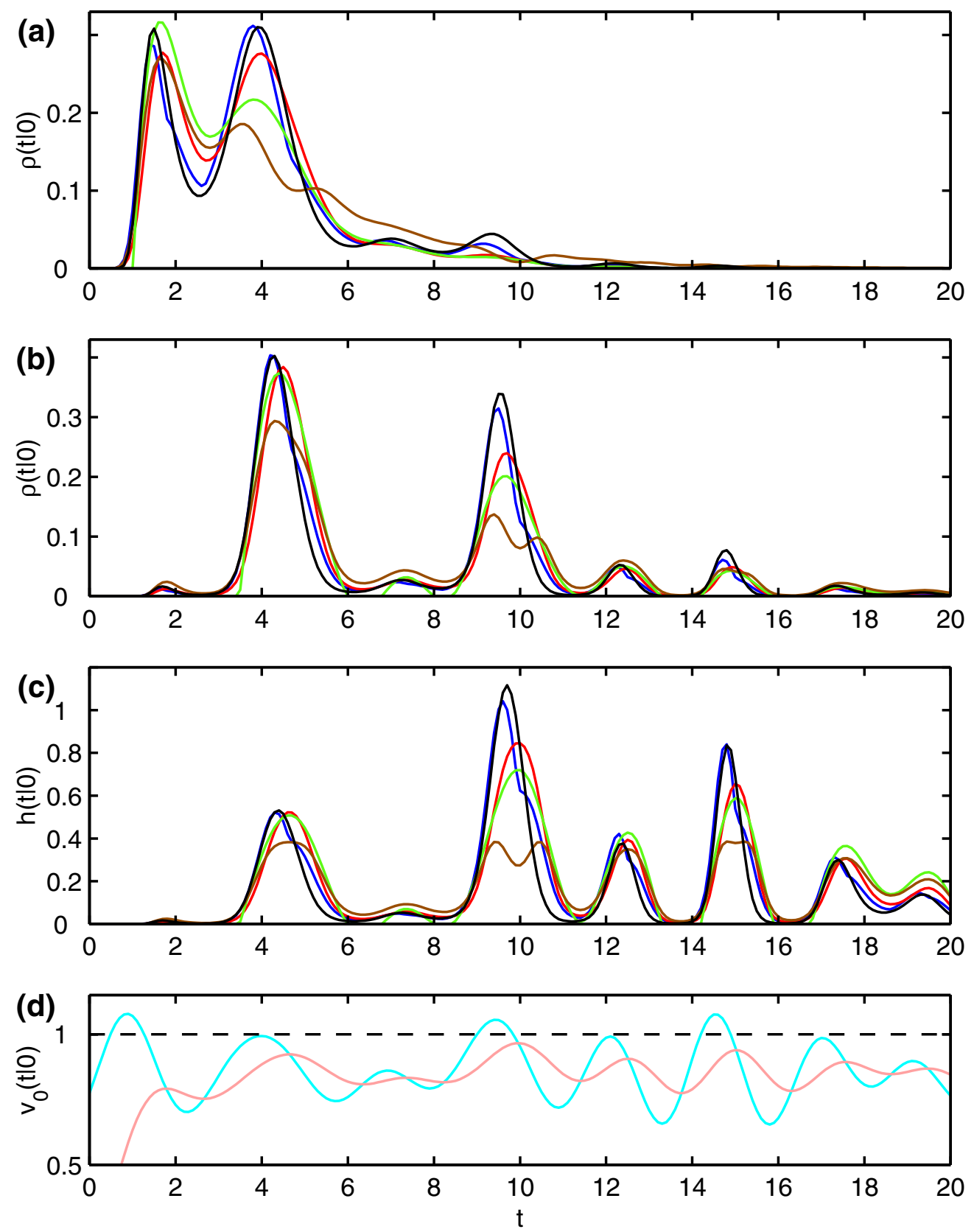

Figure 3.5: Approximations to the Ornstein-Uhlenbeck neuron: (a, b) ISI densities in response to the aperiodic input shown as cyan line in (d). Noise amplitudes are (a) $\sigma=0.2$ and (b) $\sigma=0.1$ with relative distances from threshold $\varepsilon=-0.44$ and $\varepsilon=-0.88$. The black line is the density for the OU neuron, red the Arrhenius, blue the Arrhenius\&Current, green the linear ramp, and brown the Tuckwell approximations. Errors range from $E=0.016$ for the Arrhenius\&Current model in (a) to $E=0.2$ for the Tuckwell approximation in (b). (c) Hazard functions pertaining to the ISI densities of (b). The hazard for the OU neuron (black) was obtained from the ISI density via Eq. (3.3). This hazard, as the A\&C hazard, follows the time course of the input $I(t)$ rather than that of the noise-free potential $v_{0}(t \mid 0)$. (d) Input $\mathrm{I}(\mathrm{t})$ (cyan), and noise-free potential $v_{0}(t \mid 0)$ (pink). The dashed line is the threshold. Stimulus parameters: base current $\mu=0.85$, amplitude $q=0.141$, cut-off frequency $\Omega_{c}=\pi$. 

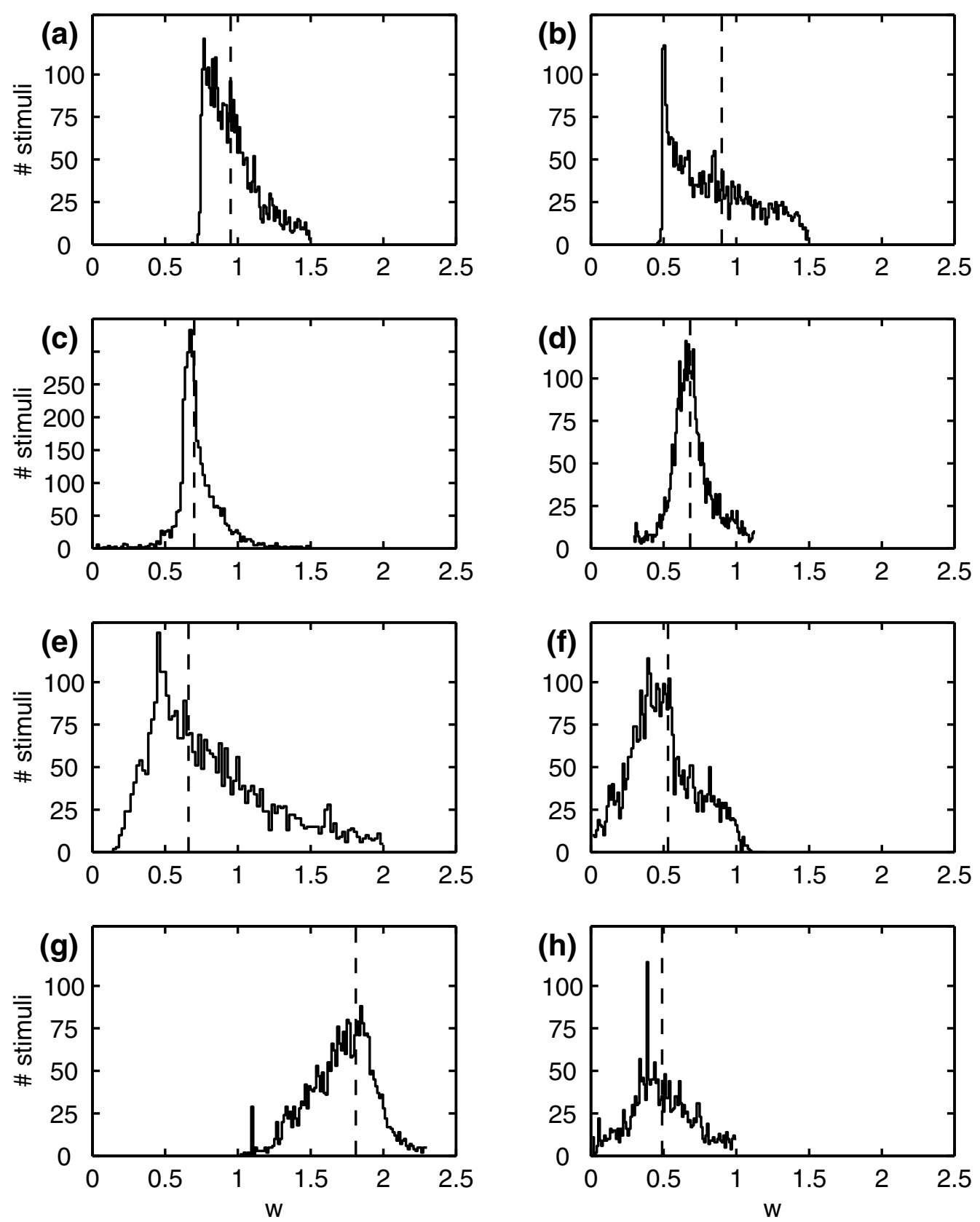

Figure 3.6: Distribution of model parameters from individual optimization of models on 4084 aperiodic and 2350 periodic stimuli: (a) Arrhenius, (b) Tuckwell, (c, d) Arrhenius\&Current, (e, f) Abeles and ( $g, h$ ) linear ramp model. For the models with two parameters, the distribution of $w_{1}$ is given in the left, that of $w_{2}$ in the right column. The dashed line marks the median, i.e. the value of $w^{\mathrm{opt}}$. 
data from 8714 periodic stimuli (out of these 4706 subthreshold stimuli) and 12689 aperiodic stimuli (8027 subthreshold) are presented here.

\subsubsection{Optimization}

As discussed in the introduction, the subthreshold regimes appears preferable for neuronal information processing. Therefore, the model parameters were optimized on the subset of non-defective subthreshold stimuli. This subset was split into an optimization and a validation set. For each one of these stimuli that parameter $w$ or parameter tuple $\left(w_{1}, w_{2}\right)$ was determined that minimized the error $E$. Since the gradient of $E$ with respect to $w$ would be extremely tedious to compute, the minimum was determined using direct search algorithms (The MathWorks, Inc. 1999). The search converged in all cases.

The distributions of the parameters obtained in this manner are shown in Fig. 3.6, together with the optimal parameter choices $w^{\text {opt }}$. The latter were chosen as the medians of the distributions. The parameters scatter rather strongly about the median with the exception of the Arrhenius\&Current model [Fig. 3.6(c), (d)]. This indicates that all other models may be adapted to individual stimuli, but that they will not perform well with fixed parameters across a wide range of stimuli.

Evaluating the error $E$ over both the optimization and the validation set with the fixed parameters $w^{\text {opt }}$ yielded identical results. Overfitting can therefore be excluded.

\subsubsection{Performance}

Figure 3.7 displays the relative error $E$ vs. the relative threshold-distance $\varepsilon$ for all approximations over the entire periodic and aperiodic stimulus sets. The method-of-images approximation is clearly the worst of all models, with errors several orders of magnitude above those of the hazard models. It behaves particularly bad for stimuli far below threshold, while it is better than the other models for some stimuli close to threshold. As shown in Fig. 3.8(a), the error for the MOI approximation drops $\sim \sigma^{-2.5}$ with the noise amplitude, i.e. the method of images yields a high noise approximation. This is easily explained: As shown in Section 3.2, the MOI Ansatz $\mathcal{P}_{b}(v, \tau \mid w, s)$ will be a solution of the Fokker-Planck equation (2.23a) if $\mathrm{e}^{\psi(\tau \mid \hat{t})}$ is constant. From Eqs. (3.14) and (2.32) one has $\mathrm{e}^{\psi(\tau \mid \hat{t})} \approx 1-\frac{2 \sqrt{2}}{\sigma} \mathrm{e}^{-\tau}$ for $t, \sigma \gg 1$, whence the required timeindependence is attained the better, the larger $\sigma$. Figures 3.8(b) and 3.8(c) demonstrate that the error of the MOI approximation correlates strongly with violations of the normalization condition $\int \rho(t) \mathrm{d} t=1$, and, albeit less clearly, with the required positivity $\rho(t) \geq 0$. The conclusion is that the method of images does not yield a robust, and therefore useful, approximation to the 

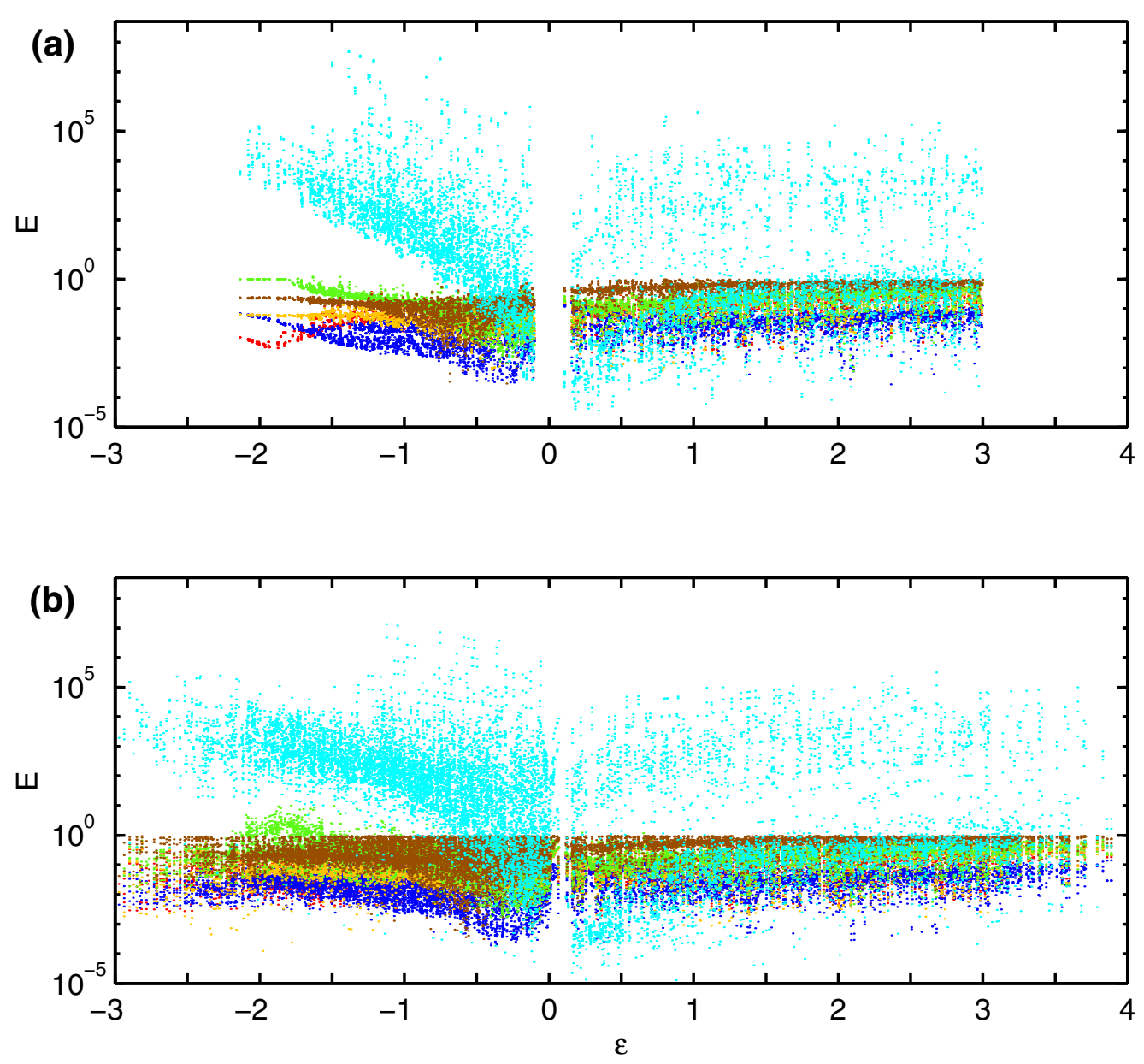

Figure 3.7: Relative error $E$ vs. relative distance from threshold $\varepsilon$ for (a) 8714 periodic and (b) 12689 aperiodic stimuli. The models are Arrhenius (red), Arrhenius\&Current (blue), Abeles (gold), linear ramp (green), Tuckwell (brown) and method-of-images (cyan). Note the logarithmic ordinate.

Ornstein-Uhlenbeck neuron. Bulsara et al. (1996) applied the approximation in the limit of strong noise $(\sigma \gg 0.1)$.

Figure 3.9 displays the relative errors of the hazard models on a larger scale. Several points may be glanced from this figure: First of all, the scatter of errors is larger for aperiodic than for periodic stimuli, particularly for stimuli far below threshold. The reason for this is that the threshold distance $\varepsilon$ characterizes aperiodic stimuli only in a root-mean-square sense. For suprathreshold stimuli, the A\&C model scores the lowest errors, while the Tuckwell model fares worst. Arrhenius, Abeles and linear ramp models are roughly tied. For subthreshold stimuli not too far below threshold $(\varepsilon \gtrsim-1.5)$, the ranking is roughly the same as above threshold, but errors tend to be smaller. For periodic stimuli far below threshold, the picture is somewhat different: the Arrhenius approxi- 

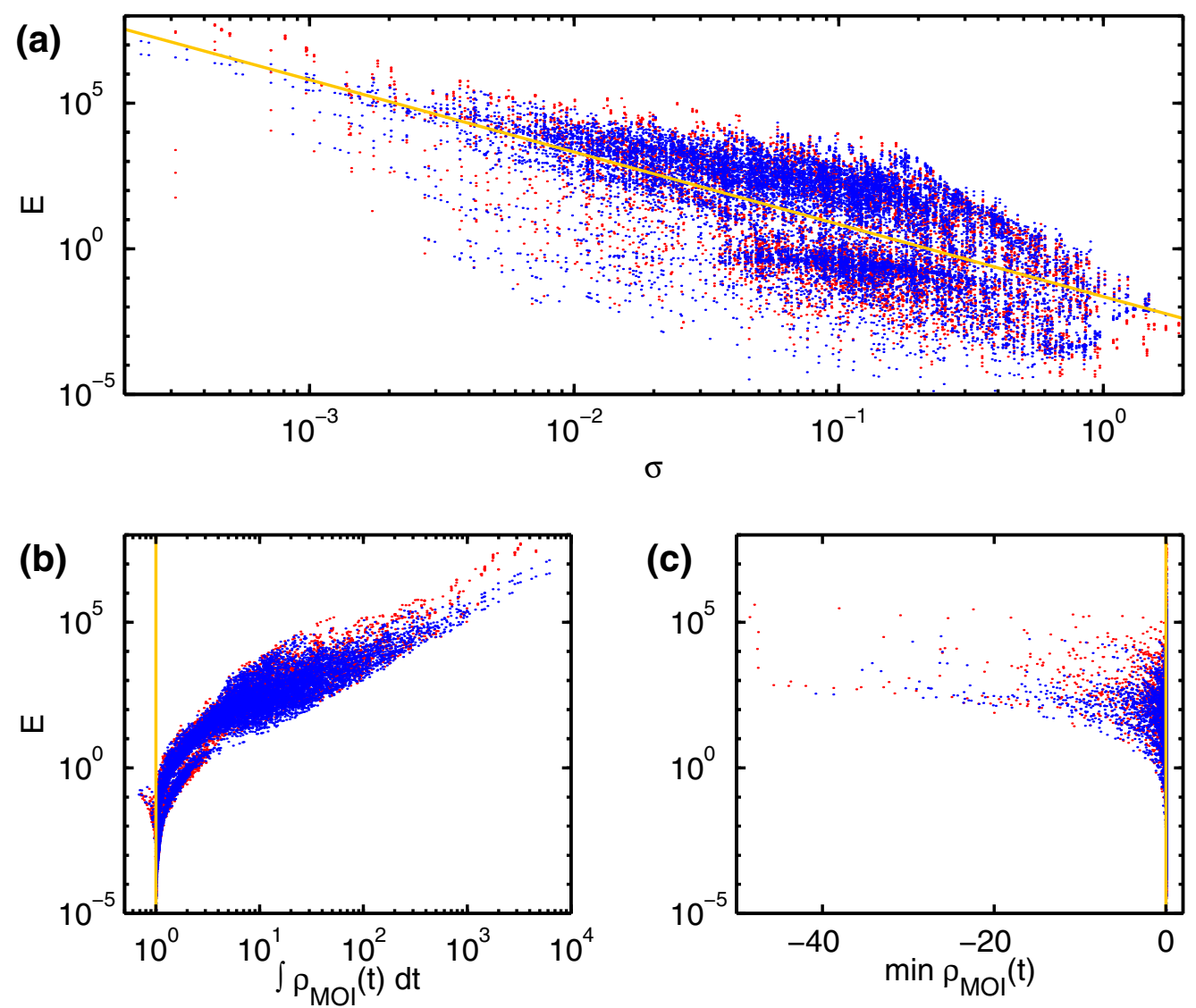

Figure 3.8: (a) The relative error $E$ of the method-of-images approximation correlates strongly with the noise amplitude $\sigma$ and (b) with norms of the ISI density greatly exceeding unity. (c) The correlation of the error to negative minima of the density is weaker. The yellow line marks the least-squares fit $E \sim \sigma^{-2.5}$ in (a), and the correct values of norm $(=1)$ and minimum $(=0)$ in (b) and (c), respectively. Red dots represent periodic, blue aperiodic stimuli.

mation now excels, since the assumptions leading to it are nearly fulfilled: the membrane potential density is virtually undistorted by the distant threshold, and transients in the stimuli shift only the exponentially small tail of the density across threshold. In this regime, the A\&C model suffers from its small coefficient $w_{1}^{\mathrm{opt}}=0.70$ for the diffusion term, compared to $w^{\mathrm{opt}}=0.95$ for the Arrhenius Ansatz. The linear model performs rather badly for subthreshold input. In particular, for $\varepsilon<-1.81$, one has $x(\tau \mid \hat{t})>1.81=-w_{1}^{\text {opt }}$ and thus $\rho_{\text {lin }}(\tau \mid \hat{t})=0$ everywhere, whence $E=1$ in this range. This cut-off causes the rise of the error for the linear model with increasing threshold-distance as well.

The error of the Tuckwell model is surprisingly large for periodic stimuli far below threshold, given that the firing rate approximation used in Eq. (3.11) is valid in precisely this regime. This is a consequence of the choice $w^{\text {opt }}=0.90$ for the model parameter, as is demonstrated in Fig. 3.10. It compares the Tuck- 

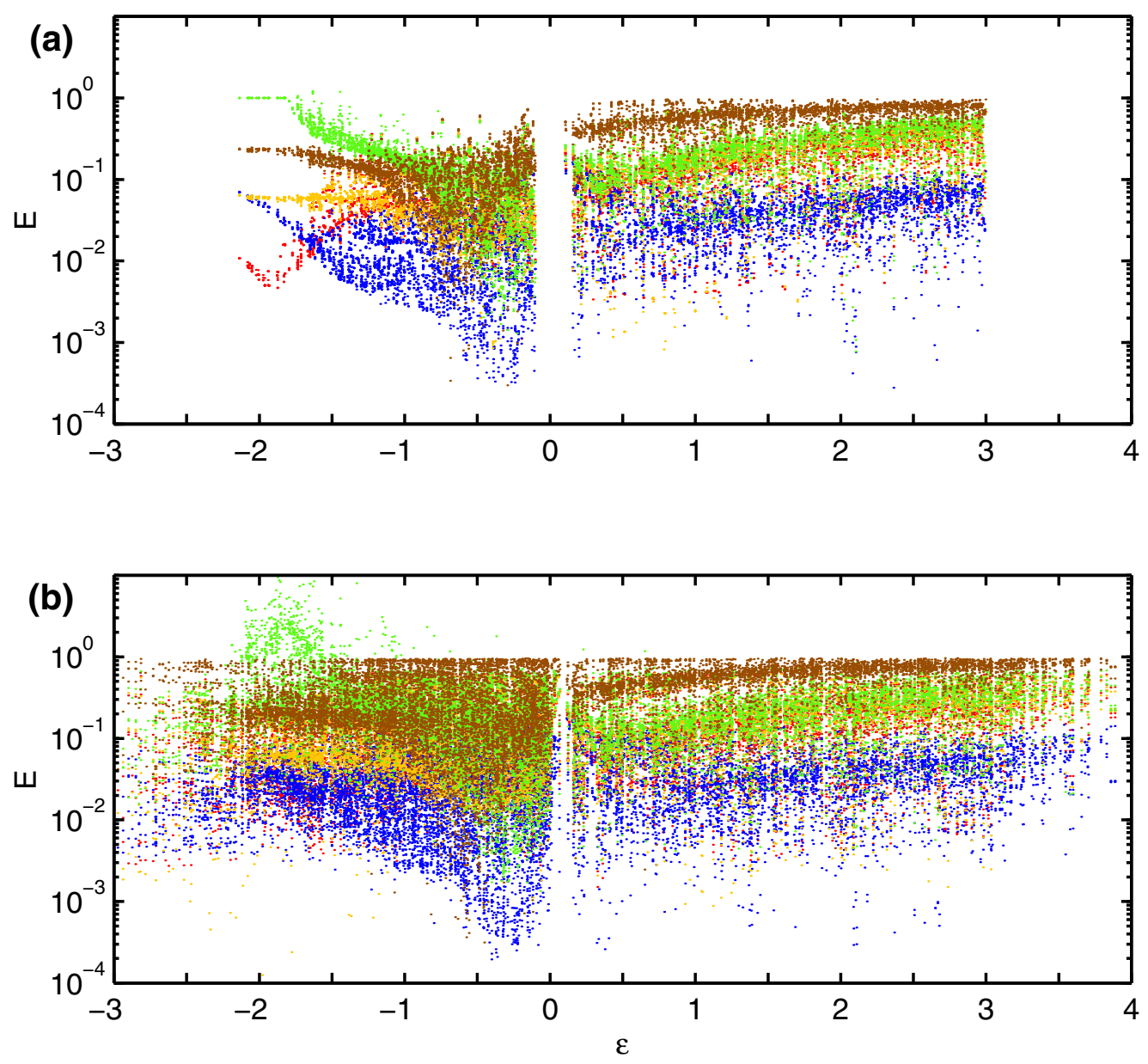

Figure 3.9: Relative error $E$ as in Fig. 3.7, but without the method-of-images data for greater clarity. The models are Arrhenius (red), Arrhenius\&Current (blue), Abeles (gold), linear ramp (green), Tuckwell (brown).

well model as defined in Section 3.1.5 to the parameter-free Tuckwell model $h_{\text {Tuck }}=[\nu(\tau \mid \hat{t})]_{+}$studied by Plesser and Gerstner (1999). This model scores smaller errors for stimuli far below threshold, but leads to larger errors once $\varepsilon \gtrsim-0.75$. It corresponds to a parameter value of $w=1 / \sqrt{\pi} \approx 0.56$. Indeed, the parameter optimization process for the Tuckwell model, see Fig. 3.6(b), indicates that a few hundred of the optimization stimuli would benefit from this choice.

Another interesting point to glance from Fig. 3.10 is that the Tuckwell model may be fine-tuned to perform well within a small range of threshold distances $\varepsilon \approx-0.5$, but that the error is quite large outside of this range. The Arrhenius model, on the other hand, does well over the entire range. This indicates that the factor $[x(\tau \mid \hat{t})]_{+}$, which is the only difference between the Arrhenius and the Tuckwell models, is the cause of the problem: it severely 


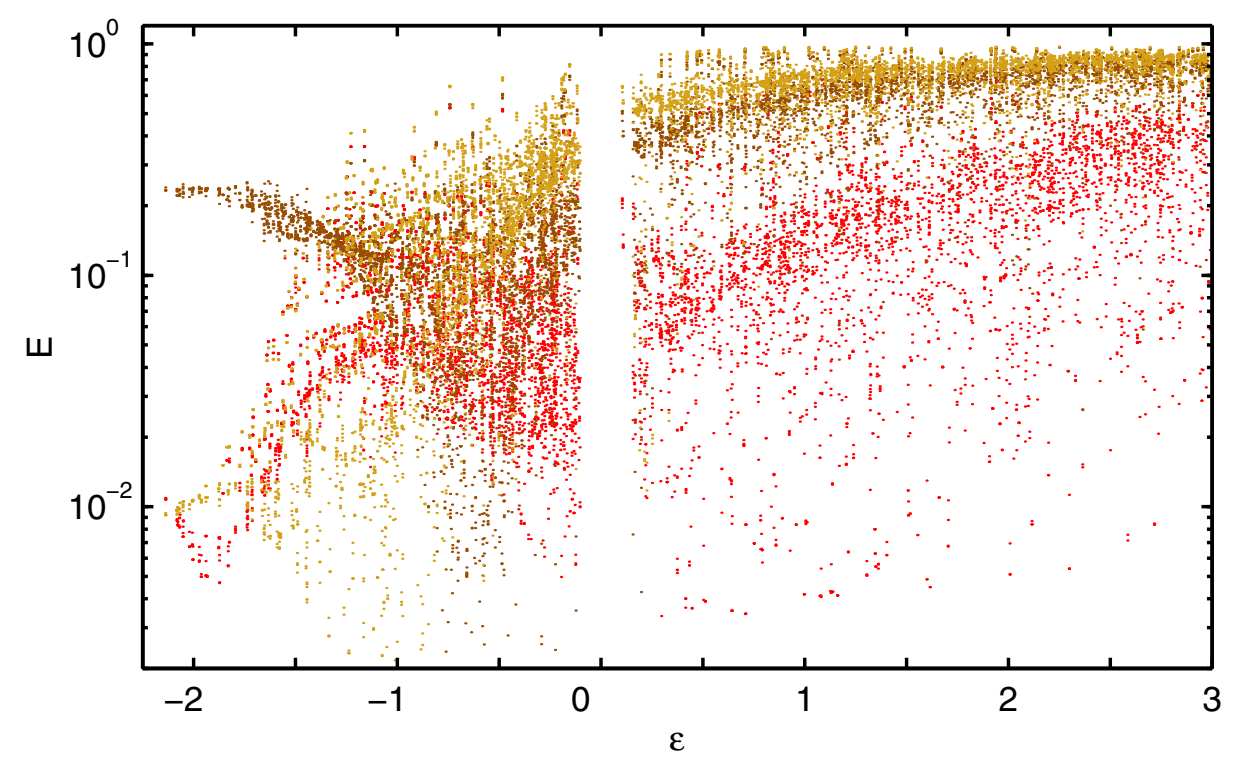

Figure 3.10: Relative error $E$ vs. distance from threshold $\varepsilon$ for the Arrhenius (red), Tuckwell (brown) and parameter-free Tuckwell model (sand) of Plesser and Gerstner (1999) for periodic stimuli.

reduces the hazard as the noise-free potential approaches the threshold, cf. Fig. 3.1(a).

Results on all approximations to the Ornstein-Uhlenbeck neuron are summarized in Fig. 3.11 and Table 3.1. The former gives the cumulative distribution of the errors $E$ shown in Fig. 3.7, distinguishing sub- and suprathreshold, and periodic and aperiodic stimuli. The Arrhenius\&Current approximation scores significantly smaller errors than all other models across all classes of stimuli, with an error $E<0.09$ for $95 \%$ of all stimuli tested. In stark contrast, the method of images yields the worst approximation overall, although it surpasses the hazard models for a small proportion of suprathreshold stimuli $(\lesssim 20 \%)$. The Arrhenius and the Abeles model are tied for second rank, again across all stimulus classes. Since the hazard functions of these two models are nearly identical for $v_{0}\left(\tau \mid v_{R}, \hat{t}\right)$ below threshold [see Fig. 3.1(a)], this vindicates the assumption that the suprathreshold portion of the hazard is rather irrelevant. The linear ramp model ties with the Tuckwell model for third rank for subthreshold stimuli, while it is nearly as good as Arrhenius and Abeles models for suprathreshold stimulation.

\subsection{Summary}

The Arrhenius\&Current model provides an excellent approximation to the Ornstein-Uhlenbeck neuron and is expected to become a valuable tool for the 

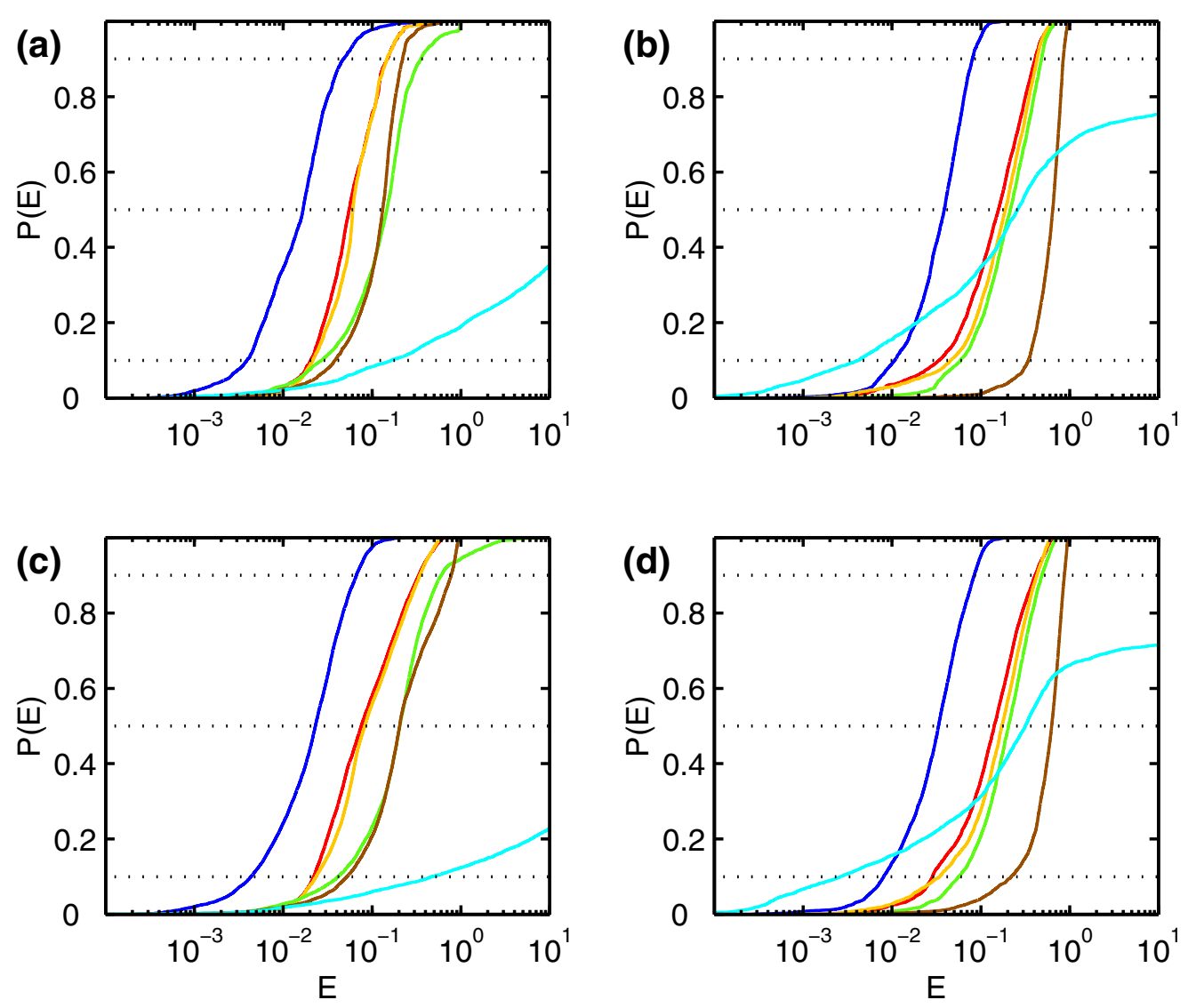

Figure 3.11: The Arrhenius\&Current model is a reliable approximation to the OrnsteinUhlenbeck neuron: Cumulative distributions $P(E)$ of the relative error periodic (top) and aperiodic stimuli (bottom). Figures on the left are for subthreshold stimuli, those on the right for suprathreshold. The models are Arrhenius (red), Arrhenius\&Current (blue), Abeles (gold), linear ramp (green), Tuckwell (brown) and method-of-images (cyan). The dashed lines mark the $10^{\text {th }}, 50^{\text {th }}$ and $90^{\text {th }}$ percentiles. Note the logarithmic abscissa.

\begin{tabular}{lrrrrr}
\hline & per, sub & per, sup & aper, sub & aper, sup & \multicolumn{1}{c}{ total } \\
\hline Arrhen. & 0.184 & 0.474 & 0.434 & 0.510 & 0.440 \\
A\&C & 0.067 & 0.096 & 0.083 & 0.099 & 0.090 \\
Abeles & 0.184 & 0.486 & 0.435 & 0.506 & 0.448 \\
ramp & 0.519 & 0.533 & 1.096 & 0.577 & 0.633 \\
Tuckw. & 0.250 & 0.890 & 0.865 & 0.913 & 0.873 \\
MOI & 23643.253 & 4166.456 & 8419.079 & 6428.771 & 8550.834 \\
\hline
\end{tabular}

Table 3.1: $95^{\text {th }}$ percentile of the approximation error $E$ for the different stimulus classes and for all classes combined (far right). Errors of the the Arrhenius\&Current approximation exceeded $E=0.09$ for only $5 \%$ of all stimuli tested. 
exploration of neuronal dynamics. It is employed in Chapter 5.3 to analyze how stochastic resonance in the OU neuron depends on the stimuli presented. It has already found applications in the analysis of network dynamics (Herrmann and Gerstner 1999). The Arrhenius and Abeles models provide reasonable approximations as well, thus justifying earlier studies based on these models a posteriori (Gerstner and van Hemmen 1992; Gerstner 1995; Abeles 1982; Spiridon and Gerstner 1999). The method of images, on the other hand, will yield useful results only under peculiar conditions.

The Arrhenius\&Current model may be of advantage even in numerical studies, e.g. when fitting model parameters to experimental data, since the computational effort required to obtain the interspike-interval density via Eq. (3.2) grows only linearly in the number of function values desired, as compared to quadratically for the Ornstein-Uhlenbeck neuron. The reduction in computer time can be dramatic.

Finally, there is some experimental evidence indicating that the Arrhenius\&Current model may be justified in its own right and not merely as an approximation to the Ornstein-Uhlenbeck neuron. Glantz (1999) has found that the firing rate of sustaining fibers of the crayfish eye is a function of both the subthreshold membrane potential and its derivative, just as for the hazard in the Arrhenius\&Current model. 


\section{Chapter 4}

\section{Spike Trains}

Neurons convey information to other neurons by sequences of stereotyped action potentials. There is mounting evidence that the temporal structure of these spike trains is an important part of the neural code (Rieke et al. 1997). Mathematical methods which predict the statistical properties of spike trains are thus a prerequisite to the exploration of the neural code. Chapters 2 and 3 forged the link between the time-dependent stimulus impinging on a neuron and the resulting distribution of interspike intervals. This chapter provides the methods required to infer the global properties of the spike train once the interspike-interval distributions are known. The methods developed here are applicable to arbitrary Markovian point processes.

Renewal processes are a subtype of Markov processes particularly amenable to analysis, since all intervals are identically distributed. Several authors have therefore opted to approximate spike trains in response to periodic stimulation as renewal processes (Plesser and Tanaka 1997; Bulsara et al. 1996). It is shown in Section 4.2 that this approximation will be valid only for peculiar stimuli. If, on the other hand, the stimulus is reset after every spike, the resulting spike train will of necessity be a renewal process. Albeit of little biological relevance, this regime of constrained stimulation is analyzed in Section 4.3. The spike intensity is introduced as a statistic of the spike train which is well-suited to illustrate - and thus understand - the response of neurons to time-dependent stimulation. It is similar to the post-stimulus time histogram measured in many neurophysiological experiments.

The Markov chain analysis of spike trains elicited by unconstrained, "natural" periodic stimulation is exposed in Section 4.4. It exploits the fact that not only the times at which spikes occur form a Markov process, but the stimulus phases at the spike times as well. The latter process is restricted to the unit circle and thus analyzed efficiently. It is shown to converge to a unique stationary distribution regardless of initial conditions (asymptotic stability). The entrainment of the neuronal firing pattern by periodic stimulation is thus completely characterized. In addition it is shown that the spike intensity can be computed quite efficiently from the Markov chain formed by the spike times. 
The most important statistics recorded in neurophysiological experiments are briefly discussed in Section 4.5 and their relation to the quantities derived here is established. Section 4.6 presents the essential methods for the investigation of stochastic resonance: The power spectral density of spike trains is derived from the interspike-interval densities via renewal process or Markov chain analysis for constrained and unconstrained stimulation, respectively.

\subsection{Definitions}

Several terms and quantities that will be used in the analysis of spike trains are defined here before embarking on the analysis itself.

The spike train has already been defined in Chapter 2.1.3 as the ordered sequence of spike times

$$
\mathbf{t}=\left[t_{1}, t_{2} \ldots\right], \quad t_{0} \equiv 0<t_{1}<\cdots
$$

The time origin is fixed at the time $t_{0} \equiv 0$ of an arbitrary spike to avoid complications that arise if the interval between the beginning of a measurement and the first spike is not an interspike interval. The reference spike is not part of the spike train.

The probability for a particular train of $n$ spikes to occur, the spike train density, is given by

$$
\Upsilon_{n}(\mathbf{t})=\operatorname{Prob}\left\{\begin{array}{l}
\text { After reference spike at } t_{0}=0, \text { the } \\
\text { neuron fires at } 0<t_{1}<\ldots<t_{n} .
\end{array}\right\} .
$$

The number of spikes is arbitrary, but will assumed to be finite to avoid unnecessary complication; this is well justified, since for any neuron the firing rate, and thus the number of spikes fired in a finite interval, is bounded. All statistical properties of the spike train, and thus of the neural response to a stimulus $I(t)$ can be computed from the spike train density. It is important to keep in mind that $\Upsilon_{n}(\mathbf{t})$ is not invariant under translations in time, unless the stimulus $I(t)$ is translated in the same way. To make this dependence on the stimulus explicit, one may want to write $\Upsilon_{n}\left(\mathbf{t} \mid I(t), t_{0}\right)$, but this is not done here for ease of notation.

The neuron models introduced in the previous chapters yield only the conditional interspike-interval densities $\rho\left(t_{k}-t_{k-1} \mid t_{k-1}\right)$, so that $\Upsilon_{n}(\mathbf{t})$ has to be obtained from these. Since the membrane potential of the neuron is reset to a fixed level $v_{R}$ after each spike, the neuron has no memory reaching back beyond the most recent spike. If the noise is without memory as well, i.e. Poissonian as in Stein's model or white Gaussian as in the Ornstein-Uhlenbeck neuron, all interspike intervals will be statistically independent. They may still depend on the times at which the intervals begin, as time-invariance is broken by the 
stimulus. The sequence of spike times is thus a Markov process and the spike time density may be decomposed as

$$
\Upsilon_{n}(\mathbf{t})=\prod_{k=1}^{n} \rho\left(t_{k}-t_{k-1} \mid t_{k-1}\right) .
$$

If the reset were not to a fixed potential $v_{R}$, but random (Bugmann et al. 1997), the ISI densities had to be conditioned on the reset potential in addition to the spike time $t_{k-1}$ to allow for the above decomposition. If the noise had finite correlation time, but were still Markovian, ${ }^{1}$ the status of the noise process at $t_{k-1}$ would be required as a further condition. For non-Markovian noise, no decomposition as given by Eq. (4.2) appears possible.

The spike train density $\Upsilon_{n}(\mathbf{t})$ is an $n$-dimensional probability density and thus difficult both to compute and to interpret. More convenient are some of its marginal densities, namely the spike time density (STD)

$$
\begin{aligned}
q_{k}(t) & =\operatorname{Prob}\left\{\text { In train } \mathbf{t}, \text { the } k^{\text {th }} \text { spike occurs at time } t .\right\} \\
& =\int \cdots \int \Upsilon_{n}\left(t_{1}, \ldots, t_{k-1}, t, t_{k+1}, \ldots, t_{n}\right) \prod_{m \neq k} \mathrm{~d} t_{m},
\end{aligned}
$$

the joint spike time density (jSTD)

$$
\begin{aligned}
q_{j+k, j}(t, s) & =\operatorname{Prob}\left\{\begin{array}{l}
\text { In train } \mathbf{t}, \text { the } j+k^{\text {th }} \text { spike } \\
\text { occurs at } t \text { and the } j^{\text {th }} \text { at } s .
\end{array}\right\} \\
& =\int \cdots \int \Upsilon_{n}\left(\ldots, t_{k-1}, s, t_{k+1}, \ldots, t_{j-1}, t, t_{j+1}, \ldots\right) \prod_{m \neq j, k} \mathrm{~d} t_{m}
\end{aligned}
$$

and the conditional spike time density (cSTD)

$$
q_{k}(t \mid s)=\operatorname{Prob}\left\{\begin{array}{l}
\text { In train } \mathbf{t}, \text { the } k^{\text {th }} \text { spike after } \\
\text { a spike at } s \text { occurs at } t .
\end{array}\right\}=q_{j+k, j}(t, s) / q_{j}(s) .
$$

The last two densities are zero if $t \leq s$ for $k>0$. The cSTD depends only on the number $k$ of spikes between the spikes at $s$ and $t$, because the train is a Markov process. Finally, the firing activity of a pool of neurons or across a number of repetitions of an experiment is given by the spike intensity ${ }^{2}$

$$
q(t)=\operatorname{Prob}\{\text { Any spike occurs at } t .\}=\sum_{k=1}^{\infty} q_{k}(t) .
$$

\footnotetext{
${ }^{1}$ E.g. exponentially correlated noise generated by an Ornstein-Uhlenbeck process.

${ }^{2}$ The spike intensity should not be confused with the conditional intensity (bedingte Intensität) studied by Rotter (1994), which is conditional on the firing history of the neuron. The intensity defined here is averaged over all possible spike trains following a reference spike at $t=0$. It is closely related to the population activity of an ensemble of independent neurons (Gerstner 1999b).
} 
The latter is not a probability density. Instead, the integral of $q(t)$ over an interval $[a, b]$ gives the average number of spikes that will occur during this time across a large number of experiment repetitions, or across a large pool of independent, identical neurons. Closed expressions for these functions in terms of the interspike-interval densities will be derived below.

Stimuli can be classified into four regimes which different mathematical treatment:

- The spike train is a proper renewal process if the stimulus is constant, $I(t)=\mu$. This case is of little relevance to neuronal signal processing, and is not pursued further in this work. It can be treated with the standard methods of renewal theory.

- The same time-dependent stimulus $I(t)$ is presented during each interval, i.e. the stimulus is reset after each spike. All intervals will thus have identical ISI densities, and the spike train is again a renewal process. This regime will be called constrained stimulation, since the stimulus presentation is constrained by the activity of the neuron.

- Stimulation with an arbitrary, time-dependent stimulus $I(t)$ will be called unconstrained stimulation. It is the regime most relevant to neurobiology. The spike train is no renewal process in this case.

- The spike train might be sufficiently similar to a renewal process even under unconstrained stimulation, e.g. if the stimulus varies extremely rapidly on the timescale of the neuron, or if the noise is very strong compared to the modulation amplitude of the stimulus. The neuron is said to fire in the approximate renewal regime. It may be analyzed in the framework of renewal theory. The validity of the renewal approximation is tested in Section 4.2.

A brief remark on terminology is in order. In a recent paper on the sources of periodic input to integrate-and-fire neurons, Lánský (1997) introduced the terms "endogenous" and "exogenous" stimulation for the constrained and unconstrained regimes defined above; this terminology has also been used by Shimokawa et al. (1999a). Lánský motivated his choice as follows: exogenous stimulation enters Stein's model (Eq. 2.4) through time-dependent pulse rates $\lambda(t)$, which cannot be reset upon a spike. Endogenous stimulation, in contrast, results from varying pulse amplitudes $\alpha(t)$, which are of postsynaptic origin and thus can be reset. This terminology has two disadvantages: First, both types of modulation lead to exactly the same form of the diffusion approximation (Chapter 2.1), and a nomenclature derived from a "model behind the model" is unsatisfactory. Second, neurons can autonomously generate firing patterns which are decidedly non-renewal, whence the implication of an internal origin of modulation evoking renewal spike trains is misleading. An example will be given in Chapter 5.2. 


\subsection{Approximate renewal trains}

If the response of the integrate-and-fire neuron to unconstrained stimulation were such that it could approximately be treated as a renewal process, the analysis would be simplified significantly. This assumption is implied in the work of Plesser and Tanaka (1997) and Bulsara et al. (1996), but its validity has never been tested systematically before.

The decision whether renewal analysis is appropriate should be based on experimental considerations. In a typical electrophysiological experiment, rarely more than a few thousand spikes are recorded per neuron and stimulus type, as the experiment duration is limited and the focus is usually on testing many neurons and various stimuli; exceptions are mostly found in insect neurophysiology (Rieke et al. 1997). Thus, if statistical tests on a train of, e.g., 10000 spikes generated by the Ornstein-Uhlenbeck neuron under unconstrained stimulation, indicate significant deviation from a renewal process, the spike train may not be treated as a renewal process.

Cox and Lewis (1966, Ch. 6.4) suggest the rank product-moment statistic

$$
R_{1}=\sum_{j=1}^{N-1} \operatorname{rk}\left(\tau_{j}\right) \operatorname{rk}\left(\tau_{j+1}\right)
$$

to test whether a sequence of intervals forms a renewal process. $N$ is the number of intervals in the sample, and $\operatorname{rk}\left(\tau_{j}\right)$ is the rank of interval $j$ in an ordered list of interval lengths. Under the null hypothesis of a renewal process, i.e. when interval durations are not correlated to each other, $R_{1}$ is asymptotically normal with mean ${ }^{3}$ and variance

$$
\begin{aligned}
\mu_{N} & =\frac{(N-1)(N+1)(3 N+2)}{12}, \\
\sigma_{N}^{2} & =\frac{(N+1)\left(5 N^{6}+21 N^{5}+501 N^{4}-823 N^{3}+1102 N^{2}-68 N+240\right)}{720(N-2)(N-3)} .
\end{aligned}
$$

The hypothesis that the spike train is a renewal process is thus rejected at significance level $\alpha$ if erfc $\left|\left(R_{1}-\mu_{N}\right) / \sigma_{N}\right|<\alpha$.

This test is applied to sinusoidal stimuli as defined by Eq. (2.42) for a wide range of frequencies $\Omega$ and noise amplitudes $\sigma$. Spike trains were obtained by direct integration of Eq. (2.20) using the algorithm given in Appendix B. Results are shown in Fig. 4.1. The response of the neuron may not be approximated by a renewal process in the large part of the parameter space marked white. The reason for this can be glanced from Fig. 4.1(d): In most stimulus periods, a small number of spikes is fired in rapid succession. These bursts are separated by intervals nearly as long as a stimulus period. Thus two types of

\footnotetext{
${ }^{3}$ The expression in Cox, p. 166 , has a prefactor of $1 / 2$ instead of $1 / 12$, which appears to be a typographical error.
} 
(a)

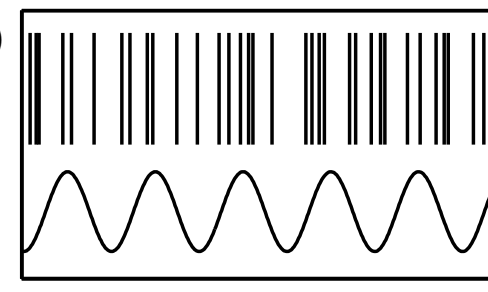

(b)

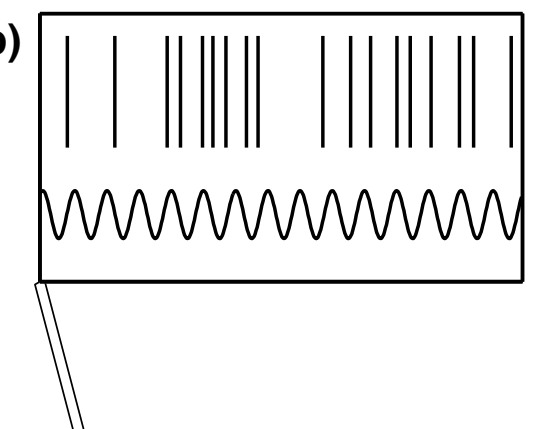

(c)

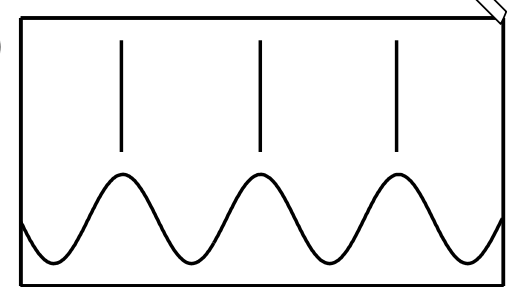

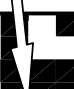
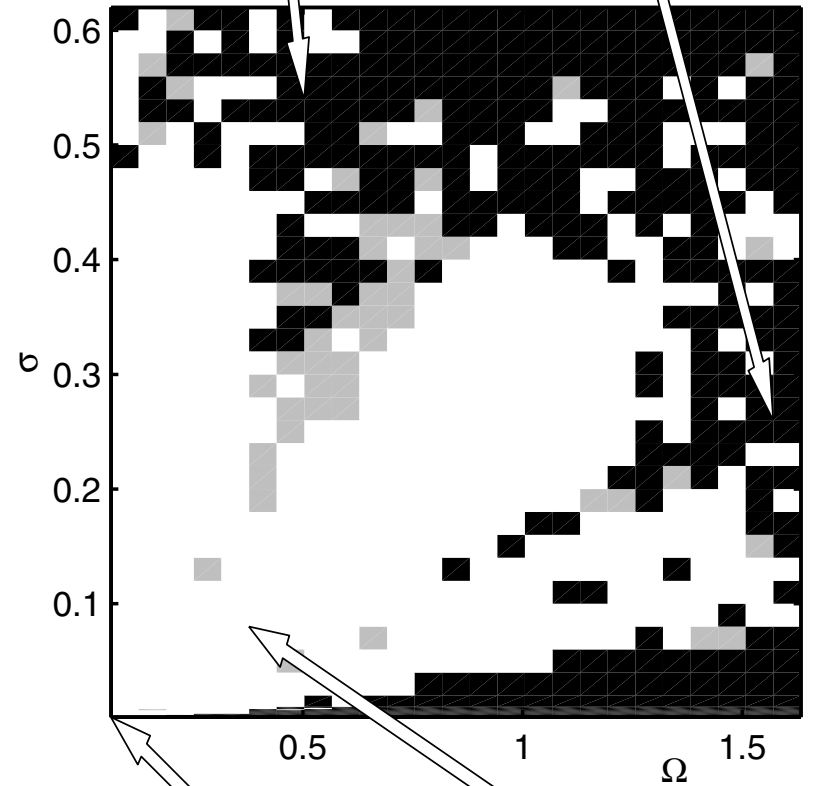

$\Omega$

Figure 4.1: The response to unconstrained periodic stimulation is a renewal process under peculiar conditions. Center: Rank product-moment test of the renewal hypothesis on trains of 10000 spikes in response to unconstrained periodic stimuli of different frequencies $\Omega$ and noise amplitudes $\sigma$. White indicates rejection of the hypothesis, i.e. non-renewal behavior, black acceptance. Grey patches mark acceptance by the rank product-moment test, but rejection by the serial correlation test (Cox and Lewis 1966, Ch. 6.4). Small figures: sample stimuli and spike trains for different stimuli as indicated by the arrows. (a) Renewal case (iii), (b) case (ii), (c) case (i), and (d) typical non-renewal spike train; see text. Stimulus amplitudes shown are $\sim 1 / \sqrt{1+\Omega^{2}}$ and frequencies are illustrative. Other parameters: $\mu=0.9, q=0.1, \phi_{0}=0$. 
intervals - intra- and inter-burst - exist, which quite obviously have very different ISI densities. The spike train cannot be treated as a renewal process therefore.

The part of the parameter space that allows for the renewal approximation can be subdivided into three regimes:

(i) Weak noise, low frequency phase-locking, Fig. 4.1(c): Spikes are locked to a stimulus phase $\phi_{\text {opt }}$ with very small jitter, entailing the required reset if the initial phase of the stimulus is $\phi_{0}=\phi_{\text {opt }}$, cf. Fig. 4.2. Since $\phi_{\text {opt }}$ shifts with noise amplitude, this condition cannot be fulfilled for fixed $\phi_{0}$; see Fig. 5.6(c), p. 89.

(ii) High frequency, Fig. 4.1(b): Due to the low pass property of the integrator neuron, the modulation of the subthreshold membrane potential is dampened $\sim 1 / \sqrt{1+\Omega^{2}}$ (cf. Eq. 2.43). This damping decouples the firing, and thus the ISI densities, from the stimulus. The neuron essentially converts constant input to random output.

(iii) Strong noise, Fig. 4.1(a): The noise dominates the stimulus, decoupling stimulus and response as in case (ii).

Cases (ii) and (iii) are not very interesting biologically, since they attain renewalness by suppressing the signal, while case (i) applies only to periodic stimuli in a regime of rather low firing rates. In conclusion, the approximation as a renewal process of the firing pattern of a neuron receiving unconstrained stimuli is valid only in cases of little practical relevance.

\subsection{Constrained stimulation}

The main motivation to discuss constrained stimulation is its mathematical elegance, and because it may serve as approximation to the true behavior of the neuron in some cases, see Section 4.2. From a biological point of view, it is highly questionable, since the author is unaware of any experimental evidence for a neuron driven by processes that are reset after every spike.

In the context of constrained stimulation, the stimulus $I(t)$ is the nominal stimulus, which may be any smooth function. Figure 4.2 demonstrates the effect of the reset after each spike on the effective stimulus that actually drives the neuron. Under favorable conditions, effective and nominal stimulus will be very similar, and the output of the neuron will reflect the periodicity of the nominal stimulus [Fig. 4.2(a)]. In other cases, though, nominal and effective stimulus may differ markedly [Fig. 4.2(b)].

In the constrained case, the interspike-interval density $\rho(\tau)$ is the same for all intervals regardless of the time at which they begin. The spike train density 
(a)

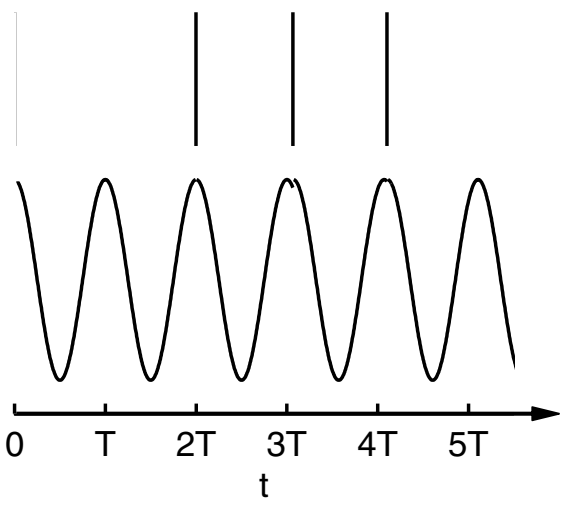

(b)
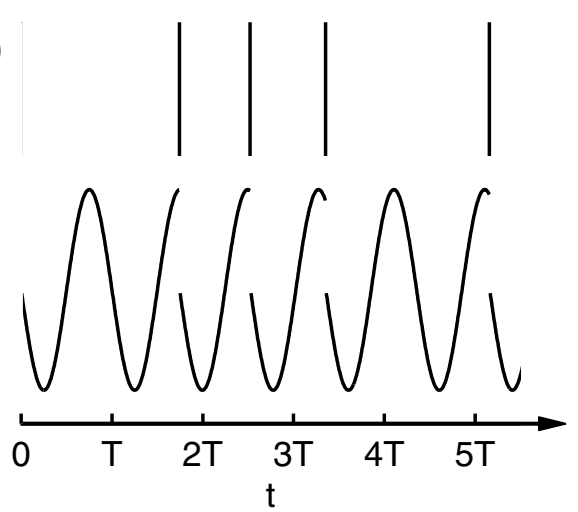

Figure 4.2: The effect of the stimulus reset depends strongly on the initial stimulus phase $\phi_{0}$ : (a) Sinusoidal stimulus with initial phase $\phi_{0}=0$. Spikes are elicited around stimulus maxima, and the reset is nearly imperceptible. The neuron effectively receives sinusoidal input. (b) The same stimulus but for $\phi_{0}=\pi / 2$. The effective stimulus is now very different from the nominal sinusoid. Parameters: $\mu=0.9, q=0.1, \Omega=0.1 \pi, \sigma=0.01$.

of Eq. (4.2) thus simplifies to

$$
\Upsilon_{n}(\mathbf{t})=\prod_{k=1}^{n} \rho\left(t_{k}-t_{k-1}\right)
$$

The spike time density is obtained by iterated convolutions

$$
q_{k}(t)=\int_{t_{0}}^{t} \rho\left(t-t_{k-1}\right) q_{k-1}\left(t_{k-1}\right) \mathrm{d} t_{k-1}, \quad q_{1}(t)=\rho(t)
$$

and Laplace transformation yields

$$
\bar{q}_{k}(s)=\bar{\rho}^{k}(s) \quad \text { with } \quad \bar{\rho}(s)=\int_{0}^{\infty} \rho(t) \mathrm{e}^{-s t} \mathrm{~d} t .
$$

The Laplace transform of the spike intensity is then a geometric sum in $\bar{\rho}(s)$ so that (Cox and Miller 1965)

$$
\bar{q}(s)=\sum_{k=1}^{\infty} \bar{q}_{k}(s)=\sum_{k=1}^{\infty} \bar{\rho}^{k}(s)=\frac{\bar{\rho}(s)}{1-\bar{\rho}(s)}, \quad|s|>0 .
$$

Franklin and Bair (1995) have shown that at the origin

$$
\lim _{s \rightarrow 0} \bar{q}(s)=\left\langle\Delta \tau^{2}\right\rangle /\langle\tau\rangle-1=C_{\mathrm{v}}^{2}-1
$$

for a mean ISI length $\langle\tau\rangle$ with variance $\left\langle\Delta \tau^{2}\right\rangle$ and corresponding coefficient of variation $C_{\mathrm{v}}$ (see Section 4.5). The spike intensity $q(t)$ is known as renewal 

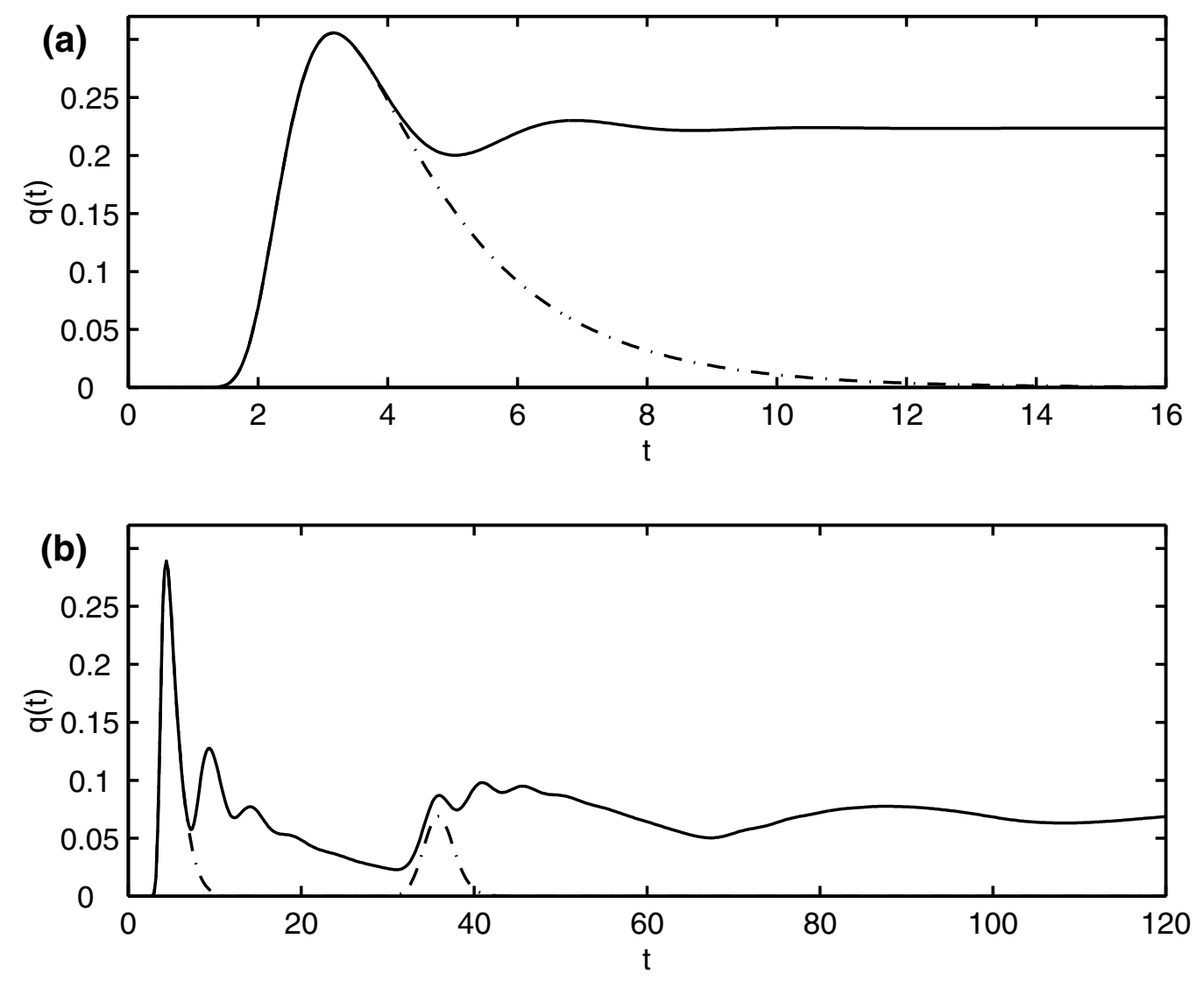

Figure 4.3: Constrained periodic stimulation does not elicit periodic firing: (a) Renewal density (solid) and ISI density (dash-dotted) for constant stimulus. The renewal density quickly becomes stationary $[\mu=0.95, \sigma=0.1]$. (b) The same for a sinusoidal stimulus with period $T=40$. The renewal density shows a complex structure which smears out only slowly $\left[\mu=0.95, q=0.05, \Omega=0.05 \pi, \phi_{0}=0, \sigma=0.02\right]$.

density in the theory of point processes. It can be obtained from Eq. (4.11a) by inverse Laplace transformation.

Figure 4.3 gives examples for constant and sinusoidal stimulation. It might come as a surprise that the renewal density is not constant even for a constant stimulus, which should give rise to stationary behavior. But homogeneity in time has been broken by locking $t_{0}=0$ to a spike. In consequence, $q(t) \approx \rho(t)$ for small $t$, and the renewal density becomes stationary only at later times as the effect of the reference spike decays. ${ }^{4}$ Periodic stimulation may evoke complex renewal densities, as shown in Fig. 4.3(b): the broad peak-trough structure with a period slightly shorter than the nominal stimulus period $T=40$

\footnotetext{
${ }^{4}$ This is known as an ordinary renewal process; all intervals are identically distributed. Equilibrium renewal processes are obtained if the distribution of the interval between $t=0$ and the first spike is the forward recurrence time distribution. They have flat renewal densities; see Cox and Miller (1965, Ch. 9).
} 
is caused by the "periodic" peak of the ISI density at $t \approx 35$. The superimposed fine structure arises from the sharp "refractory" peak of the ISI density at $\tau \approx 4.5$, but is quickly washed out. The observed complex structure has been verified by simulated spike trains (see Appendix B). At larger times, the spike intensity smoothes markedly. Indeed, one can show that $q(t) \rightarrow 1 /\langle\tau\rangle$ for large times (Cox and Miller 1965). This is a consequence of the stimulus reset: Since the neuron fires at random times, the mean effective stimulus at large times is a superposition of randomly shifted copies of $I(t)$, equivalent to constant input.

\subsection{Unconstrained stimulation}

The biologically most relevant stimulus regime, unconstrained stimulation that may not be approximated as a renewal process, poses considerable technical difficulties. Each interspike interval has a different distribution $\rho(\tau \mid t)$, conditional on the time $t$ of its beginning. Therefore, the spike time density is no longer given by a sequence of proper convolutions as in Eq. (4.9). Instead, one has

$$
q_{k}(t)=\int_{0}^{t} \rho\left(t-t_{k-1} \mid t_{k-1}\right) q_{k-1}\left(t_{k-1}\right) \mathrm{d} t_{k-1}, \quad q_{1}(t)=\rho(t)
$$

and likewise for the conditional spike time density

$q_{k}(t \mid s)=\int \ldots \int_{0}^{t} \rho\left(t-t_{k-1} \mid t_{k-1}\right) \rho\left(t_{k-1}-t_{k-2} \mid t_{k-2}\right) \ldots \rho\left(t_{1}-s \mid s\right) \prod_{j=1}^{k-1} \mathrm{~d} t_{j}$.

Because the integration variables $t_{j}$ appear in the conditioning argument of $\rho$ as absolute time, the spike time densities cannot be obtained via Laplace transforms. Not even the mean ISI duration $\langle\tau\rangle$ is accessible, as its computation requires proper weighting of all conditional ISI densities.

If the stimulus is periodic, the Markov process of spike times on the positive real axis may be reduced to a Markov process of the stimulus phases at which spikes occur. This process is confined to the unit circle, and is easily characterized by a stochastic (Markov) operator. Asymptotic results for the latter provide the desired information about the stationary firing patterns. The discretization of the operator to a stochastic matrix is straightforward, leading to efficient numerics for the resulting Markov chain. The Markov operator and pertaining results are introduced below, and the Markov chain is discussed in Section 4.4.2. The spike intensity is obtained from the infinite Markov chain in time in Section 4.4.3. 


\subsubsection{Continuous phase: Markov kernel}

Let $T$ be the period of the stimulus, i.e. the smallest $T$ with $I(t+T)=I(t)$ for all $t$, and define the stimulus phase as

$$
\psi=\hat{\Omega} t \bmod 2 \pi,
$$

with the pseudo-frequency $\hat{\Omega}=2 \pi / T$. This definition of phase is entirely general: In particular, the stimulus may be an arbitrary superposition of sinusoids with rational periods $T_{j} \in \mathbb{Q}$. The stimulus period is then the least common multiple of the individual periods, whence the term pseudo-frequency for $\hat{\Omega}$. Since the conditional ISI densities depend on absolute time only through the stimulus (compare Eqs. 2.33, 2.31), the periodicity of the stimulus carries over to the conditioning argument of the conditional ISI density. With $\hat{t}=n T+\psi / \Omega$ one has

$$
\rho(\tau \mid \hat{t})=\rho(\tau \mid n T+\psi / \Omega)=\rho(\tau \mid \psi / \Omega) \equiv \rho(\tau \mid \psi) .
$$

The last equality above is a minor re-definition of $\rho$ to obtain a more compact notation. Thus, the conditional ISI densities for $\psi \in[0,2 \pi)$ suffice to describe neural spike trains. Furthermore, it follows from Eq. (4.15) that the sequence of spike phases

$$
\boldsymbol{\psi}=\left[\psi_{1}, \psi_{2}, \ldots\right], \quad \psi_{k}=\hat{\Omega} t_{k} \bmod 2 \pi, \quad t_{k} \in \mathbf{t}
$$

is a Markov process just as the spike train $\mathbf{t}$ itself; $\psi_{0}=0$ is the phase of the reference spike. In analogy to the spike time density, the spike phase density (SPD) is defined as

$$
\begin{aligned}
\chi_{k}(\psi) & =\operatorname{Prob}\left\{\text { In train } \mathbf{t}, \text { the } k^{\text {th }} \text { spike occurs at phase } \psi \cdot\right\} \\
& =\frac{1}{\hat{\Omega}} \int_{0}^{\infty} q_{k}(t) \delta(\psi-\hat{\Omega} t \bmod 2 \pi) \mathrm{d} t \\
& =\frac{1}{\hat{\Omega}} \sum_{n=0}^{\infty} q_{k}\left(n T+\frac{\psi-\psi_{0}}{\Omega}\right) .
\end{aligned}
$$

The Markov process $\boldsymbol{\psi}$ formed by the spike phases is mathematically much more manageable than the full spike train $\mathbf{t}$, as it is restricted to $[0,2 \pi)$. The evolution of the spike phase density is captured by the Markov kernel

$$
\begin{aligned}
T(\psi \mid \phi) & =\operatorname{Prob}\{\text { A spike at phase } \phi \text { is followed by a spike at phase } \psi \cdot\} \\
& =\frac{1}{\hat{\Omega}} \int_{0}^{\infty} \rho(\tau \mid \phi) \delta(\psi-[\hat{\Omega} \tau+\phi] \bmod 2 \pi) \mathrm{d} \tau \\
& =\frac{1}{\hat{\Omega}} \sum_{n=0}^{\infty} \rho\left(n T+\frac{\psi-\phi}{\hat{\Omega}} \mid \phi\right) .
\end{aligned}
$$



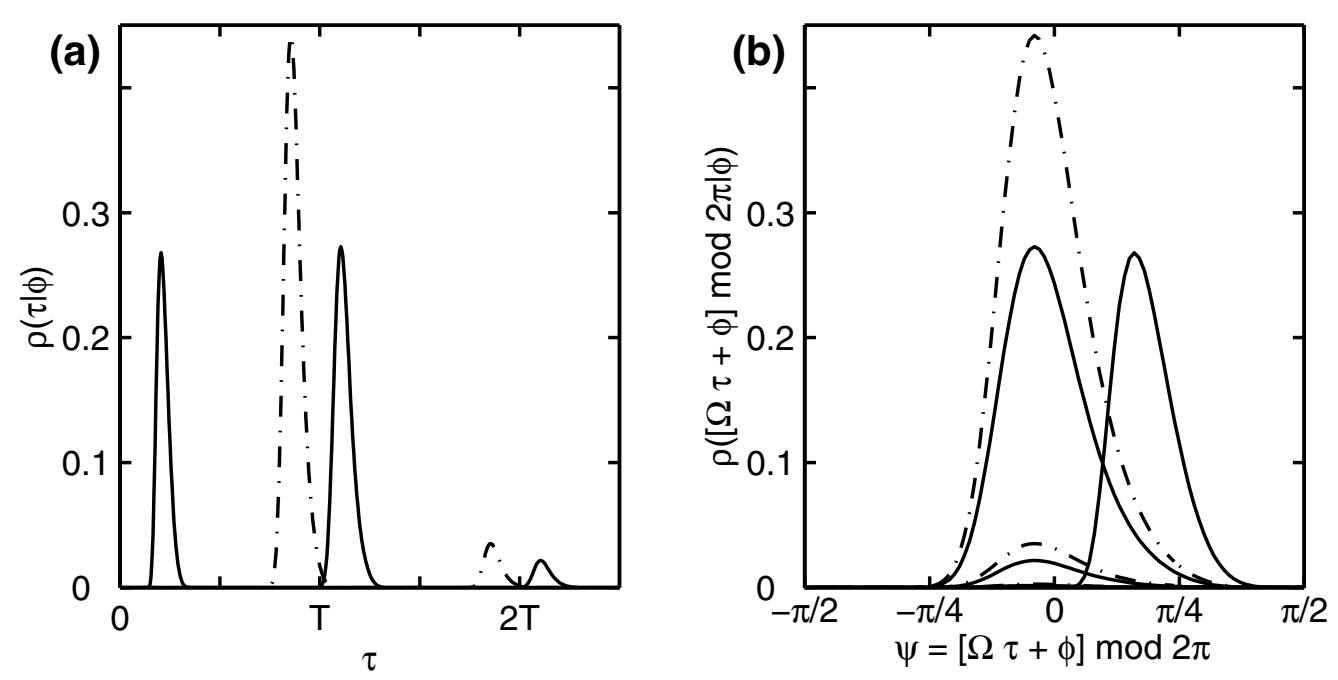

Figure 4.4: Construction of the Markov kernel: (a) Conditional ISI densities in response to a sinusoidal stimulus for phases $\phi=-\pi / 4$ (solid) and $\phi=\pi / 4$ (dash-dotted). The conditional ISI density for $\phi=-\pi / 4$ has a refractory peak at $\tau \approx T / 4$, and two periodic peaks near $T$ and $2 T$, while for $\phi=\pi / 4$, only periodic peaks exist. (b) Same densities as in (a), but vs. stimulus phase $\psi=\Omega \tau+\phi \bmod 2 \pi$. All periodic peaks are at the same location with respect to phase, and the refractory peak is clearly set apart. The kernel value $T(\psi \mid \phi)$ is obtained by summing the contributions from all periods at phase $\psi$ for given $\phi$. Other parameters: $\mu=0.9, q=0.1, \Omega=0.1 \pi, \sigma=0.02$.

The series above converges, since the conditional ISI density is normalized, implying $\rho(\tau \mid \psi)<c / \tau^{1+\alpha}$ for $\tau \rightarrow \infty$ and some $\alpha>0$, so that the MacLaurinCauchy criterium is fulfilled. ${ }^{5}$ Intuitively, the kernel value $T(\psi \mid \phi)$ is obtained by summing across all periods the probability of firing at phase $\psi$ for an interval beginning at phase $\phi$ as displayed in Fig. 4.4. Note that the probability to fire at a certain phase may be qualitatively different in different stimulus periods.

By construction, the Markov kernel is a probability density in its first argument $\psi$ conditional on the second $\phi$, i.e.

$$
\int_{0}^{2 \pi} T(\psi \mid \phi) \mathrm{d} \psi=1 \quad \text { and } \quad T(\psi \mid \phi) \geq 0 .
$$

Such kernels are called stochastic kernels. For arbitrary probability densities $f(\psi)$ on $[0,2 \pi)$, i.e. functions with $f(\psi) \geq 0$ and $\int_{0}^{2 \pi} f(\psi) \mathrm{d} \psi=1$, the stochastic kernel $T$ defines an integral operator via

$$
(\mathcal{T} f)(\psi) \equiv \int_{0}^{2 \pi} T(\psi \mid \phi) f(\phi) \mathrm{d} \phi .
$$

If follows immediately from Eq. (4.19) that $\mathcal{T} f$ is again a density. Therefore $\mathcal{T}$ is a Markov operator mapping probability densities defined on $[0,2 \pi)$ onto each

\footnotetext{
${ }^{5}$ Since $\rho(\tau \mid \psi)=0$ for $\tau<0$, the summand $n=0$ is well-defined for $\psi<\phi$.
} 
other. Markov operators are a generalization of Frobenius-Perron operators and have been studied intensely in the field of chaotic dynamics (Lasota and Mackey 1994).

The evolution of the spike phase density is thus given by

$$
\chi_{k+1}(\psi)=\mathcal{T} \chi_{k}(\psi)=\mathcal{T}^{k+1} \chi_{0}(\psi)
$$

where the initial distribution $\chi_{0}(\psi)$ is chosen by the experimentalist: If the stimulus sets in with a fixed phase $\phi_{0}$ at the reference spike, one has $\chi_{0}(\psi)=$ $\delta\left(\psi-\phi_{0}\right)$; if the onset phase is chosen from a uniform distribution, the initial distribution is $\chi_{0}(\psi)=1 / 2 \pi$. The conditional spike phase density, defined in analogy to the conditional spike time density, is given by the kernel $T_{k}(\psi \mid \phi)$ of the operator $\mathcal{T}^{k}$, i.e.

$$
\chi_{k}(\psi \mid \phi)=\operatorname{Prob}\left\{k^{\text {th }} \text { spike after spike at } \phi \text { occurs at } \psi \cdot\right\}=T_{k}(\psi \mid \phi)
$$

with $\chi_{1}(\psi \mid \phi)=T(\psi \mid \phi)$ by definition.

Phase locking of neuronal spikes to a stimulus, as observed in the auditory pathway, is often characterized by cycle histograms, discrete correlates of the spike phase density $\chi$ (Rose et al. 1967). Such a histogram, compiled from spike trains recorded over many stimulus periods, will show a well defined structure -i.e. locking - only if the firing pattern of the neuron is stationary with respect to stimulus phase, with the possible exception of a brief transient after stimulus onset. Therefore, the asymptotic behavior of the phase density $\chi_{k}$ is of great interest.

Loosely speaking, one would like to show that the sequence of phase densities converges to a stationary density

$$
\chi_{k} \longrightarrow \chi^{(s)}=\mathcal{T} \chi^{(s)} \quad \text { for } \quad k \rightarrow \infty
$$

regardless of the initial density $\chi_{0}$. This convergence is more precisely defined by the notion of asymptotic stability: For a Markov operator $\mathcal{T}$, the sequence $\left\{\mathcal{T}^{k}\right\}$ is said to be asymptotically stable, if a unique density $f^{*}$ on $[0,2 \pi)$ exists with $\mathcal{T} f^{*}=f^{*}$ and

$$
\lim _{k \rightarrow \infty}\left\|\mathcal{T}^{k} f-f^{*}\right\|=0 \quad \text { for all densities } f \text {. }
$$

$\|x\|$ is the $L^{1}$ norm on $[0,2 \pi)$. The Markov operator $\mathcal{T}$ defined by the kernel $T(\psi \mid \phi)$ of Eq. (4.18) is asymptotically stable by virtue of Corollary 5.7.1 of Lasota and Mackey (1994), since

$$
\int_{0}^{2 \pi} \inf _{\phi \in[0,2 \pi)} T(\psi \mid \phi) \mathrm{d} \psi \geq \min _{\psi, \phi \in[0,2 \pi)} T(\psi \mid \phi)>0 .
$$

Strict positivity holds because $\rho(\tau \mid \phi)$ is strictly positive, as was proven in Chapter 2.2. Thus, the spike phase density of the Ornstein-Uhlenbeck neuron 
will converge to a unique stationary density $\chi^{(s)} \psi$ for any initial phase density. This is the first strict proof of asymptotic stability. Other authors had merely conjectured that Eq. (4.24) held (Tateno et al. 1995; Shimokawa et al. 1999a).

Once the phase density for the $k^{\text {th }}$ spike is known, the interspike interval density for the $k+1^{\text {st }}$ spike is

$$
\rho^{(k+1)}\left(\tau \mid \psi_{0}\right)=\int_{0}^{2 \pi} \rho(\tau \mid \psi) \chi_{k}(\psi) \mathrm{d} \psi
$$

and for the stationary state follows

$$
\rho^{(s)}(\tau)=\int_{0}^{2 \pi} \rho(\tau \mid \psi) \chi^{(s)}(\psi) \mathrm{d} \psi
$$

\subsubsection{Discrete phase: Markov chain}

To facilitate numerical treatment, the phase axis is discretized. Since the conditional interspike-interval densities $\rho(\tau \mid \psi)$ are smooth in both time and phase due to the presence of noise in the input, this discretization will introduce only minor numerical errors. It is largely equivalent to applying numerical methods to solve the eigenvalue problem for the Markov operator defined by Eq. (4.23) (Baker 1977). Using $L$ bins of width $\Delta \psi(\Delta \psi=2 \pi / L)$, the spike phase density is replaced by a distribution vector

$$
\boldsymbol{\chi}=\left(\boldsymbol{\chi}_{0}, \boldsymbol{\chi}_{1}, \ldots, \boldsymbol{\chi}_{L-1}\right)^{\mathrm{tr}}, \quad \boldsymbol{\chi}_{j}=\int_{\left(j-\frac{1}{2}\right) \Delta \psi}^{\left(j+\frac{1}{2}\right) \Delta \psi} \chi(\psi) \mathrm{d} \psi
$$

Indices run from zero to $L-1$ for notational convenience. Likewise, the Markov operator $\mathcal{T}$ turns into a matrix $\mathbf{T}$ with elements ${ }^{6}$

$$
\begin{aligned}
\mathbf{T}_{j k} & =\frac{1}{\Delta \psi} \int_{\left(j-\frac{1}{2}\right) \Delta \psi}^{\left(j+\frac{1}{2}\right) \Delta \psi} \int_{\left(k-\frac{1}{2}\right) \Delta \psi}^{\left(k+\frac{1}{2}\right) \Delta \psi} T(\psi \mid \phi) \mathrm{d} \phi \mathrm{d} \psi \approx \int_{\left(j-\frac{1}{2}\right) \Delta \psi}^{\left(j+\frac{1}{2}\right) \Delta \psi} T(\psi \mid k \Delta \psi) \mathrm{d} \psi \\
& =\frac{1}{\hat{\Omega}} \sum_{n=0}^{\infty} \int_{\left(j-\frac{1}{2}\right) \Delta \psi}^{\left(j+\frac{1}{2}\right) \Delta \psi} \rho\left(n T+\frac{\psi-k \Delta \psi}{\hat{\Omega}} \mid k \Delta \psi\right) \mathrm{d} \psi, \quad j, k=0, \ldots, L-1 .
\end{aligned}
$$

The approximation made above is to estimate the integral over the conditional argument $\phi \in\left[\left(k-\frac{1}{2}\right) \Delta \psi,\left(k+\frac{1}{2}\right) \Delta \psi\right)$ by the value at the bin center $\phi=k \Delta \psi$. This is dictated by numerical efficiency, since the conditional ISI densities are computationally expensive, and a high resolution of the phase axis desired. Thus one will not want to compute more than $L$ ISI densities just to "waste" them as midpoints in the integration. Errors are negligible for smooth kernels.

\footnotetext{
${ }^{6} T(\psi \mid \phi)$ is not a density in $\phi$, requiring the normalization by $\Delta \psi$.
} 
A brief comment on notation: vectors are represented by bold lowercase letters, while matrices are written as bold uppercase letters. The elements of a matrix $\mathbf{A}$ are given as $\mathbf{A}_{j k}$, and likewise for vectors. If functions were indexed in the continuous case, such as the spike phase densities $\chi_{k}$, this index is written as superscript in parentheses, $\chi^{(k)}$. All vectors are column vectors.

The iteration equation (4.21) simplifies to a matrix-vector multiplication

$$
\chi^{(k+1)}=\mathbf{T} \cdot \chi^{(k)}
$$

and the stationary distribution $\chi^{(s)}$ is the eigenvector to eigenvalue 1 of the matrix T. The Markov process on the unit circle is thus reduced to a Markov chain with $L$ states. All results for Markov operators have counterparts in the theory of Markov chains (Feller 1970, Ch. XV-XVI). In particular, since the kernel of the Markov operator is strictly positive, one has $\mathbf{T}_{j k}>0$. Finite Markov chains with this property are irreducible and aperiodic, and have a unique eigenvector to eigenvalue $\lambda=1$; see von Mises (1964, Ch. 12.3). All other eigenvectors have eigenvalues $|\lambda|<1$. This means that the convergence property of the Markov operator carries over to the Markov chain so that

$$
\chi^{(k)} \longrightarrow \chi^{(s)}=\mathbf{T} \cdot \chi^{(s)} \quad \text { for } \quad k \rightarrow \infty
$$

for any initial phase distribution $\chi^{(0)}$.

In practice, the transition matrix $\mathbf{T}$ is obtained numerically via Eq. (4.18) from the conditional ISI densities $\rho(\tau \mid \phi)$ of Chapters 2 and 3. The stationary distribution is then found using standard eigenvector routines (The MathWorks, Inc. 1998). The stationary ISI distribution finally is given by Eq. (4.26). This process is not without numerical perils: While the column-wise normalization of $\mathbf{T}$ can be enforced, ${ }^{7}$ many matrix elements may be zero to numerical precision. Indeed, numerical stability is often improved by setting matrix elements on the order of the machine accuracy to zero. This might yield a "numerical matrix" that is no longer irreducible and aperiodic. But since the stationary density $\chi^{(s)}$ of the Markov operator $\mathcal{T}$ is guaranteed to exist and to be unique, it is safe to take the eigenvector to eigenvalue 1 of $\mathbf{T}$ as stationary distribution $\chi^{(s)}$. This holds as long as a only single eigenvalue fulfills $|\lambda|=1$ and the eigenvector computation is numerically stable. A rigorous test against simulations is given in Section 4.6.2, see Fig. 4.11, p. 75 .

An example for the phase evolution of an initially uniform phase distribution towards the stationary state under the influence of a transition matrix $\mathbf{T}$ is given in Fig. 4.5. The phase axis was divided into $L=72$ intervals of $\Delta \psi=\pi / 36=5^{\circ}$. To "read" the transition matrix, note that the matrix columns correspond to the phase $\phi$ of the spike preceding the interval, the

\footnotetext{
${ }^{7}$ The error is negligible if the norm of the numerical ISI densities is nearly unity, e.g. $\int \rho(\tau \mid \phi) \mathrm{d} \tau>0.999$.
} 

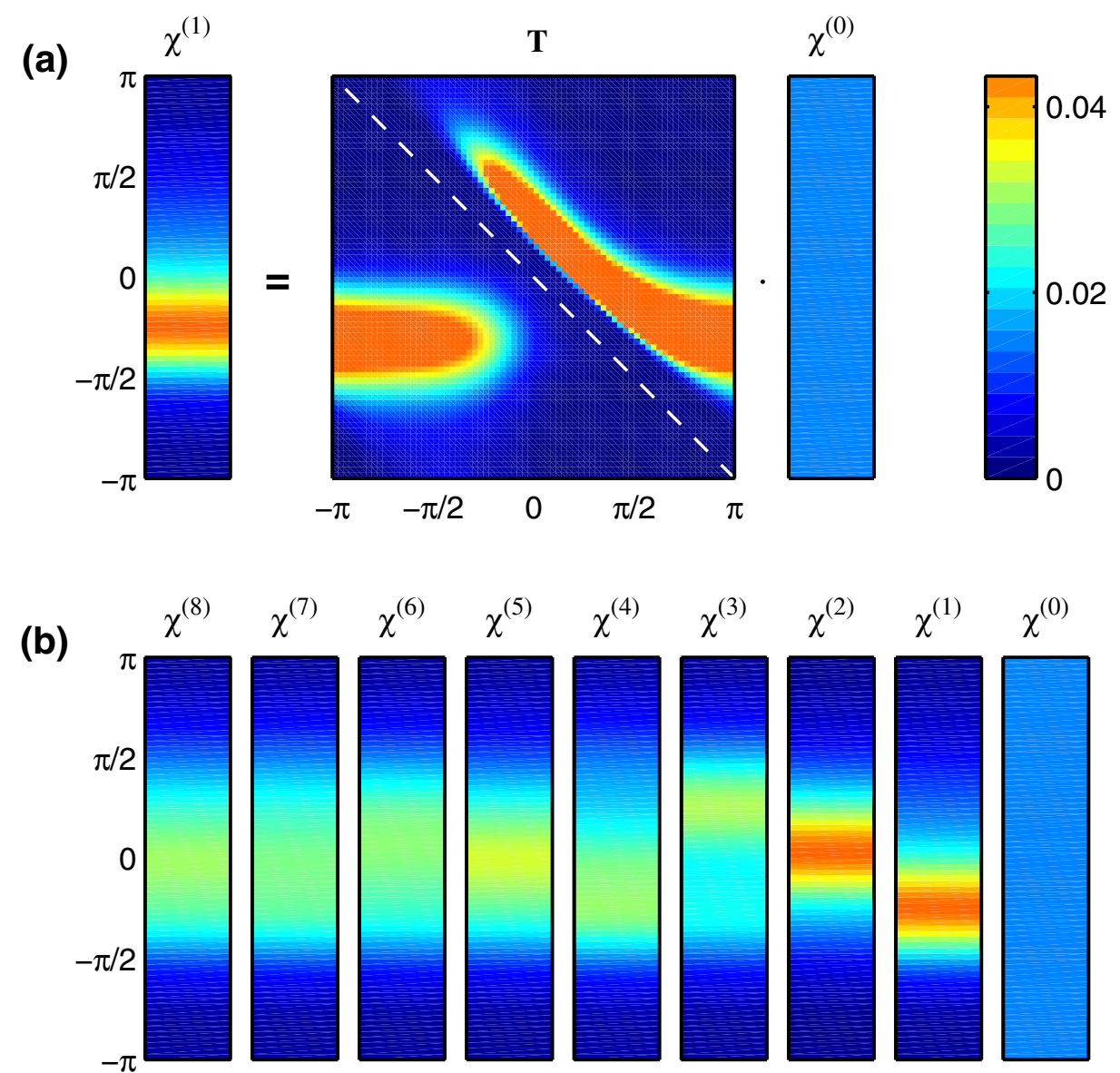

Figure 4.5: (a) Graphic representation of the Markov chain iteration given by Eq. (4.29). The dashed line is the matrix diagonal. Probability is given by color as indicated. (b) Evolution of an initially uniform phase distribution under subsequent multiplications with $\mathbf{T}$, from right to left. The approach to the stationary state is evident. See text for details. Parameters are the same as in Fig. 4.1(d): $\mu=0.9, q=0.1, \Omega=0.1 \pi, \sigma=0.1$.

rows to the phase $\psi$ of the spike terminating it. The phase axes ${ }^{8}$ run from $-\pi$ to $\pi$ from bottom to top in phase distribution vectors $\chi$ and the rows of the transition matrix $\mathbf{T}$, and from right to left across the columns of $\mathbf{T}$. Thus, the horizontal bar in the transition matrix shown in Fig. 4.5 indicates that for most values of $\phi$, the next spike will occur around $\psi \approx-\pi / 4$. The bar corresponds to the periodic peaks of the conditional ISI densities. For $-\pi / 4 \lesssim \phi \lesssim \pi / 2$, the matrix is dominated by a "finger", running parallel to the matrix diagonal. This finger results from the refractory peak of the conditional ISI distributions, which is present only in this range of $\phi$ values, compare Fig. 4.4. Within this range of phases, a spike will be followed by another spike at a slightly later phase, as shown in Fig. 4.5(b). Figuratively speaking, the neuron fires a burst

\footnotetext{
${ }^{8} \phi, \psi \in[-\pi, \pi)$ instead of $[0,2 \pi)$ renders the matrix structures more clearly.
} 
of spikes upon every period of the stimulus, compare Fig. 4.1(d). One should keep in mind, though, that there is always a chance that two subsequent spikes will be one or more stimulus periods apart, even though they are close in phase: in the Markov chain in phase, all information about actual interval lengths is lost.

\subsubsection{Markov chain in time}

Since the spike train is a Markov process, it may be treated as a Markov chain in discrete time. In contrast to the Markov chain in phase, this chain will not be limited to a finite interval, but extend along the positive real axis. Furthermore, since no two spikes can occur at the same time, all states of this Markov chain will be transient, and no stationary density of spike times exists: the time of the $n+1^{\text {st }}$ spike will have a different, "later" distribution than that of the $n^{\text {th }}$. This entails a number of difficulties: the transition matrix of the Markov chain in time is infinite, and, lacking stationarity, Eq. (4.12) for the spike intensity remains cumbersome to evaluate. The additional effort is well invested, since the spike intensity provides much insight into the stimulus response of the neuron.

Assume that conditional interspike-interval densities have been computed for a phase discretization $\Delta \psi=2 \pi / L$ as in the previous section. This $\Delta \psi$ corresponds to a time discretization of $\Delta t=T / L$, where $T=2 \pi / \hat{\Omega}$ is the stimulus period. In analogy to the phase transition matrix $\mathbf{T}$ one may now define a time transition matrix

$$
\begin{aligned}
\mathbf{Q}_{j m} & \equiv \rho((j-m) \Delta t \mid(m \bmod L) \Delta \psi) \Delta t \\
& \approx \text { Prob\{Spike at } m \Delta t \text { followed by spike at } j \Delta t .\}, \quad j, m \geq 0
\end{aligned}
$$

The columns of $\mathbf{Q}$ are discrete probability distributions with normalization $\sum_{j=0}^{\infty} \mathbf{Q}_{j m}=1$, whence $\mathbf{Q}$ is a stochastic matrix. Furthermore, it is a lower triangular matrix with vanishing diagonal, i.e. $\mathbf{Q}_{j m}=0$ for $j \leq m$. The elements above the diagonal vanish since the spikes are ordered, and the vanishing diagonal entries reflect the fact that no two spikes can occur at the same time.

The discrete counterpart to the spike time density $q_{k}(t)$ is the infinite vector $\mathbf{q}^{(k)}$ with elements

$$
\mathbf{q}_{j}^{(k)} \approx q_{k}\left(j \Delta t \mid t_{0}\right) \Delta t=\operatorname{Prob}\left\{k^{\text {th }} \text { spike occurs in }[j \Delta t,(j+1) \Delta t) .\right\} .
$$

It is a discrete probability distribution like the colums of $\mathbf{Q}$. Since the reference spike occured at $t_{0}=0$ with certainty one has

$$
\mathbf{q}^{(0)}=(1,0,0, \ldots)^{\operatorname{tr}} .
$$

Starting from this initial distribution, all following are obtained by iterated multiplication with the transition matrix

$$
\mathbf{q}^{(k)}=\mathbf{Q} \mathbf{q}^{(k-1)}=\mathbf{Q}^{k} \mathbf{q}^{(0)}
$$



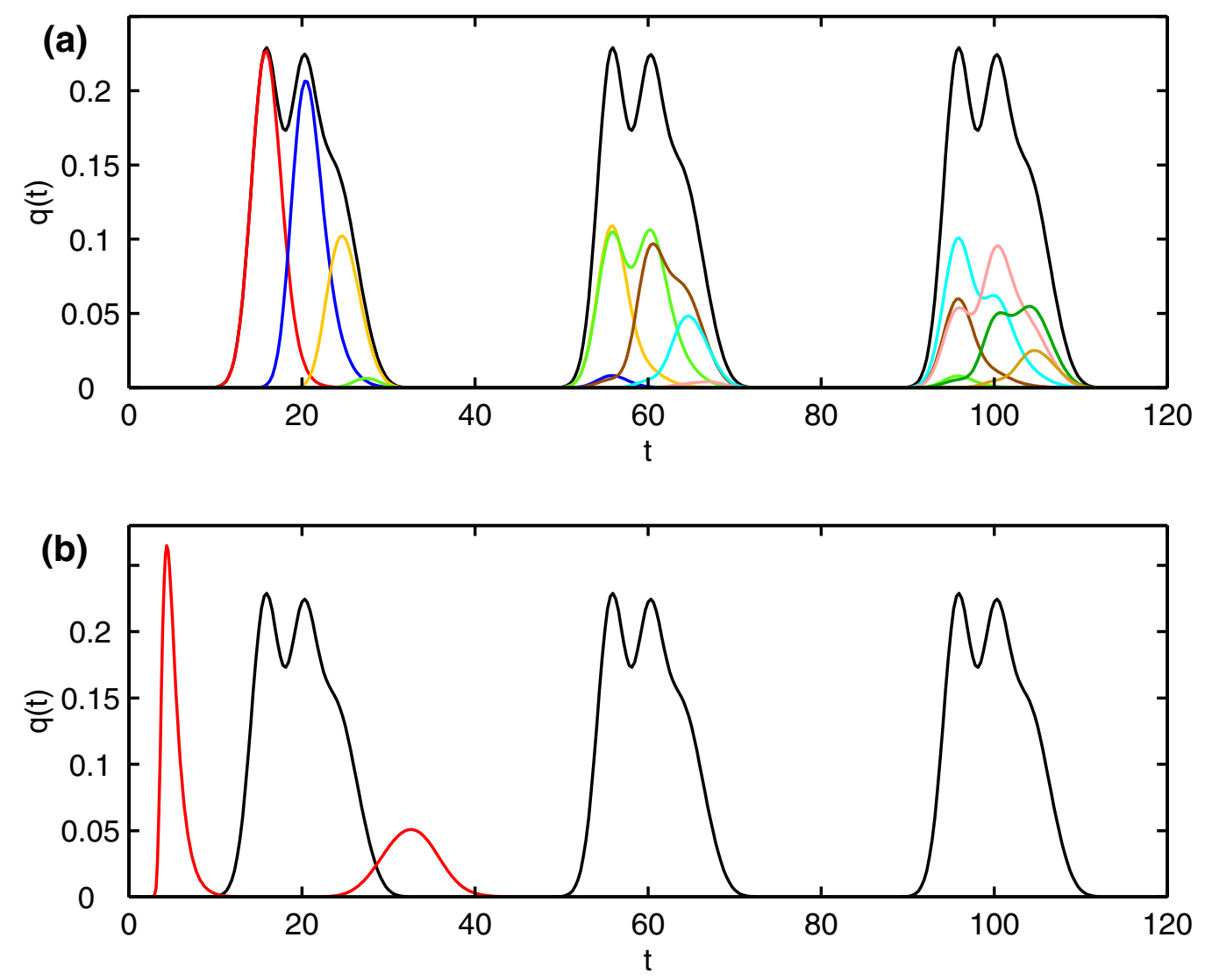

Figure 4.6: Firing activity in response to unconstrained sinusoidal stimulation clearly reflects the stimulus period $T=40$. (a) Spike intensity $q(t)$ (black) and spike time densities $q_{k}(t)$ for first (red), second (blue) to ninth spike (sand). The stimulus is the same as the nominal stimulus in Fig. 4.3(b) . (b) Spike intensity (black), and stationary ISI density $\rho^{(s)}$ (red) for the same stimulus.

which is the discrete version of Eq. (4.12). The spike intensity is thus from Eq. (4.6)

$$
\mathbf{q}=\frac{1}{\Delta t} \sum_{k=1}^{\infty} \mathbf{q}^{(k)}=\left(\sum_{k=1}^{\infty} \mathbf{Q}^{k}\right) \frac{\mathbf{q}^{(0)}}{\Delta t}
$$

Division by $\Delta t$ ensures that $\mathbf{q}_{j}$ is the probability per unit time to observe a spike around $j \Delta t$.

Numerical evaluation of these equations is facilitated by the lower triangular structure of the transition matrix $\mathbf{Q}$. This entails that all elements of the powers of $\mathbf{Q}$ with indices $j, m \leq N$ will only depend on elements of $\mathbf{Q}_{j m}$ with $j, m \leq N$. Therefore, spike trains of finite length $t_{k} \leq T_{\max }=N \Delta t$ can be treated without further approximations using $N \times N$ matrices. Furthermore, the finite transition matrices are nilpotent so that $\mathbf{Q}^{N}=0$. The infinite sum 
in Eq. (4.34) thus becomes finite and yields

$$
\mathbf{q}=\left(\sum_{k=1}^{N-1} \mathbf{Q}^{k}\right) \frac{\mathbf{q}^{(0)}}{\Delta t}=\left[(\mathbf{Q}-\mathbf{1})^{-1}-\mathbf{1}\right] \frac{\mathbf{q}^{(0)}}{\Delta t}
$$

The matrix inversion is easily performed by forward substitution leading to a recursion for the spike intensity (Press et al. 1992)

$$
\begin{array}{ll}
x_{0}=1 / \Delta t, & x_{m}=\sum_{j=0}^{m-1} \mathbf{Q}_{m j} x_{j}, \quad m \geq 1 \\
\mathbf{q}_{0}=0 & \mathbf{q}_{m}=x_{m} .
\end{array}
$$

$\mathbf{q}_{0}=0$ since the reference spike at $t_{0}=0$ is excluded from the spike train by definition.

As an example, Fig. 4.6(a) shows the spike intensity $q(t)$ and the spike time density $q_{k}(t)$ for the same stimulus as used in Fig. 4.3(b) but now for unconstrained stimulation. The difference is striking: the spike intensity strongly reflects the periodicity of the unconstrained stimulus, while the analogous renewal density of the constrained regime smoothes out quickly. In the unconstrained case, the intensity $q(t)$ is nearly stationary across periods, even though the individual $q_{k}(t)$ smear out for larger $k$. The neuron fires bursts of two to four spikes per stimulus period, interrupted by silence: the firing pattern is stochastically locked to the stimulus. Fig. 4.6(b) compares the the stationary ISI density $\rho^{(s)}(t)$ to the spike intensity and clearly demonstrates that much information about the spike train is lost if only $\rho^{(s)}(t)$ were measured. While the initial peak of the stationary ISI density indicates spikes in rapid succession, and the soft bump at $t \approx 35$ points to periods of silence between such bursts, the precise structure of the firing pattern could not be recovered.

\subsection{Spike train statistics}

Over the past decades, a set of statistics has evolved which is commonly measured in neurophysiological experiments. The most detailed characterization of the spike sequences elicited from a neuron would be given by the spike train density $\Upsilon_{n}(\mathbf{t})$. The latter is an $n$-dimensional probability density which is difficult to estimate (Scott 1992). Two approaches - mostly used in information theoretic analysis of neuronal activity - are to collect gargantuan amounts of data from insects (Strong et al. 1998) or to apply intricate correction schemes to scarce primate data (Golomb et al. 1997). Even if the spike train density could be measured efficiently, it would most likely provide little insight by itself, since it is difficult to visualize.

Interspike-interval histograms (ISIH) are much more easily obtained and have been in use for a long time (Kiang 1965). They are equivalent to the 


\begin{tabular}{lcc}
\hline & constrained & unconstrained \\
\hline PST histogram & $\mathrm{n} / \mathrm{a}$ & (spike intensity, see text) \\
ISI histogram & $\rho(\tau)$ & $\rho^{(s)}(\tau)$ \\
cycle histogram & $\sum_{k} \rho([2 \pi k+\psi] / \Omega)$ & $\chi^{(s)}(\psi)$ \\
mean ISI $\langle\tau\rangle$ & $\int_{0}^{\infty} \tau \rho(\tau) \mathrm{d} \tau$ & $\int_{0}^{\infty} \tau \rho^{(s)}(\tau) \mathrm{d} \tau$ \\
firing rate $\nu$ & $1 /\langle\tau\rangle$ & $1 /\langle\tau\rangle$ \\
coeff. of var. $C_{\mathrm{v}}$ & $\sqrt{\left\langle\Delta \tau^{2}\right\rangle /\langle\tau\rangle}$ & $\sqrt{\left\langle\Delta \tau^{2}\right\rangle /\langle\tau\rangle}$ \\
Fano factor $F$ & $C_{\mathrm{v}}^{2}$ & see $\mathrm{Eq} .(4.36)$ \\
vector strength $r$ & $\left|\int_{0}^{\infty} \rho(\tau) \mathrm{e}^{i \Omega \tau} \mathrm{d} \tau\right|$ & $\left|\int_{0}^{2 \pi} \chi^{(s)}(\psi) \mathrm{e}^{i \psi} \mathrm{d} \psi\right|$ \\
\hline
\end{tabular}

Table 4.1: Spike train statistics under constrained and unconstrained stimulation.

ISI density $\rho(\tau)$ in the case of constrained stimulation. For unconstrained stimulation, it is to be compared to the stationary ISI density $\rho^{(s)}(\tau)$ from Eq. (4.26). An example is given in Fig. 5.10, p. 94. For sinusoidal stimuli, Rose et al. (1967) introduced the cycle histogram, which is equivalent to the stationary phase distribution as discussed in Section 4.4.1.

The post-stimulus time histogram (PSTH) commonly recorded in neurophysiological experiments is related to the spike intensity $q(t)$. The essential difference between PSTH and spike intensity lies in the time origin. When measuring PSTHs, the time origin is chosen arbitrarily, and the stimulus sets in a fixed time later. For the spike intensity, the origin is fixed to a spike at or after the stimulus onset. Because of the dependence of the spike intensity on the stimulus phase $\psi_{0}$ at the time of the reference spike, only spikes in a narrow window around $\psi_{0}$ may be used as reference. This severely curtails the available data, most likely to a degree that makes it impossible to measure spike densities in in vivo experiments. A possible experimental paradigm in vitro would be to record from the neuron in the absence of a stimulus until a spike is fired, and to begin the injection of a stimulating current with a predefined phase immediately afterwards.

Essential scalar statistics are the mean interspike-interval length $\langle\tau\rangle$, its variance $\left\langle\Delta \tau^{2}\right\rangle$ and the coefficient of variation $C_{\mathrm{v}}=\sqrt{\left\langle\Delta \tau^{2}\right\rangle} /\langle\tau\rangle$. A Poisson process has $C_{\mathrm{v}}=1$, and smaller values indicate more regular spike trains. The averages above are taken over $\rho(\tau)$ for constrained and $\rho^{(s)}(\tau)$ for unconstrained stimulation. The firing rate is usually approximated as $\nu=1 /\langle\tau\rangle$. 
Another quantity that is sometimes studied is the Fano factor ${ }^{9} F$, which is defined as the ratio of variance to mean of the number of spikes fired in a time window of length $T_{o}$. For a renewal process, i.e. constrained stimulation, one has $F=C_{\mathrm{v}}^{2}$ (Zador 1998). For unconstrained stimulation the Fano factor is given by

$$
F=\left\langle N_{T_{o}}^{2}-\left\langle N_{T_{o}}\right\rangle^{2}\right\rangle /\left\langle N_{T_{o}}\right\rangle \quad \text { with } \quad\left\langle N_{T_{o}}^{k}\right\rangle=\sum_{N=0}^{\infty} N^{k} p_{N}\left(T_{o}\right)
$$

where

$$
\begin{aligned}
p_{N}\left(T_{o}\right) & =\operatorname{Prob}\left\{N \text { spikes occur in }\left(0, T_{o}\right] .\right\} \\
& = \begin{cases}1-\int_{0}^{T_{o}} q_{1}(t) \mathrm{d} t & \text { for } N=0, \\
\int_{0}^{T_{o}} q_{N}(t)-q_{N+1}(t) \mathrm{d} t & \text { for } N>0\end{cases}
\end{aligned}
$$

is the spike count distribution. A large Fano factor indicates that it is difficult to predict the number of spikes that will occur in a given time $T_{o}$.

For responses to sinusoidal stimulation, Goldberg and Brown (1969) have introduced the vector strength ${ }^{10}$ defined as

$$
r=\left|\left\langle\mathrm{e}^{i \psi}\right\rangle\right|
$$

where $\psi$ is the phase at which spikes occur. Figuratively, each spike is assigned a unit vector with direction corresponding to the spike phase, and the vector strength is the length of the sum of all these vectors, divided by the number of spikes. Thus, perfect phase-locking corresponds to $r=1$, while $r=0$ does not necessarily indicate a random firing pattern. If, e.g., two spikes were fired per stimulus period at phases $\psi=0$ and $\pi$, the vector strength is zero although the firing pattern is highly regular. For constrained stimulation, the above expectation is obtained from the ISI density $\rho(\tau)$, and from the stationary phase density $\chi^{(s)}$ otherwise. All statistics are listed in Table 4.1 along with the formulae for both stimulation regimes.

\subsection{Power spectral density}

The power spectral density (PSD) is widely used in the theory of signal processing to evaluate the transmission properties of systems. It is particularly

\footnotetext{
${ }^{9}$ In the terminology of point processes, the Fano factor is a statistic of counts, as opposed to a statistic of intervals, such as the mean ISI length.

${ }^{10}$ Plesser and Geisel (1999) referred to the vector strength $r$ as coefficient of synchronization $C_{\mathrm{S}}$, but the latter term has been defined in a different way by Rose et al. (1967). Another term for $r$ commonly used in the literature is synchronization index (Anderson et al. 1971).
} 
well suited to judge the transmission of periodic stimuli in terms of the signalto-noise ratio. The latter is the most commonly used quantity employed in investigations of stochastic resonance (Gammaitoni et al. 1998) and will play a key role in Chapter 5. Applications in neurophysiology have apparently been rare (Bair et al. 1994), except in conjunction with stochastic resonance. The power spectral density for both constrained (Plesser and Tanaka 1997) and unconstrained (Plesser and Geisel 1999) is derived here from the pertaining ISI densities.

Power spectra are often computed in the limit of an infinitely long signal, but this appears to be of little relevance to a neuroscience study: The main concern in this case is how much information neuron A can transmit to neuron $\mathrm{B}$ within a limited observation time $T_{o}$ (Stemmler 1996). This observation time may be set by the need to react quickly to stimuli, but also by the "forgetfulness" of the recipient neuron. Therefore, the time-limited power spectral density

$$
S_{T_{o}}(\omega)=\frac{1}{\pi T_{o}}\left\langle\left|\int_{0}^{T_{o}} f_{\mathbf{t}}(t) \mathrm{e}^{-i \omega t} \mathrm{~d} t\right|^{2}\right\rangle
$$

of the spike train $\mathbf{t}$ is used in this work (Priestley 1996). For a train of $\delta$-spikes the PSD becomes

$$
S_{T_{o}}(\omega)=\frac{1}{\pi T_{o}}\left\langle\sum_{0<t_{j}, t_{m} \leq T_{o}} \mathrm{e}^{-i \omega\left(t_{j}-t_{m}\right)}\right\rangle .
$$

The sums above are awkward to evaluate if the time limit, but not the number of spikes is fixed. Therefore, spike-count-conditional PSDs are computed separately for each number $M$ of spikes in $T_{o}$

$$
S_{T_{o}, M}(\omega)=\frac{1}{\pi T_{o}}\left\langle\sum_{j, k=1}^{M} \mathrm{e}^{-i \omega\left(t_{j}-t_{m}\right)}\right\rangle=\frac{1}{\pi T_{o}}\left[M+2 \operatorname{Re} \sum_{j=1}^{M-1} \sum_{k=1}^{M-j}\left\langle\mathrm{e}^{-i \omega\left(t_{j+k}-t_{j}\right)}\right\rangle\right] .
$$

These are then averaged over the spike count distribution to obtain the full power spectral density

$$
S_{T_{o}}(\omega)=\sum_{M=0}^{\infty} S_{T_{o}, M}(\omega) p_{M}\left(T_{o}\right)
$$

The evaluation of the remaining average poses major difficulties, since it is to be taken conditional on $M$ spikes occurring in $T_{o}$ : instead of averaging over the joint spike time density $q_{j+k, j}(t, s)$ defined in Eq. (4.4), one has to use the 

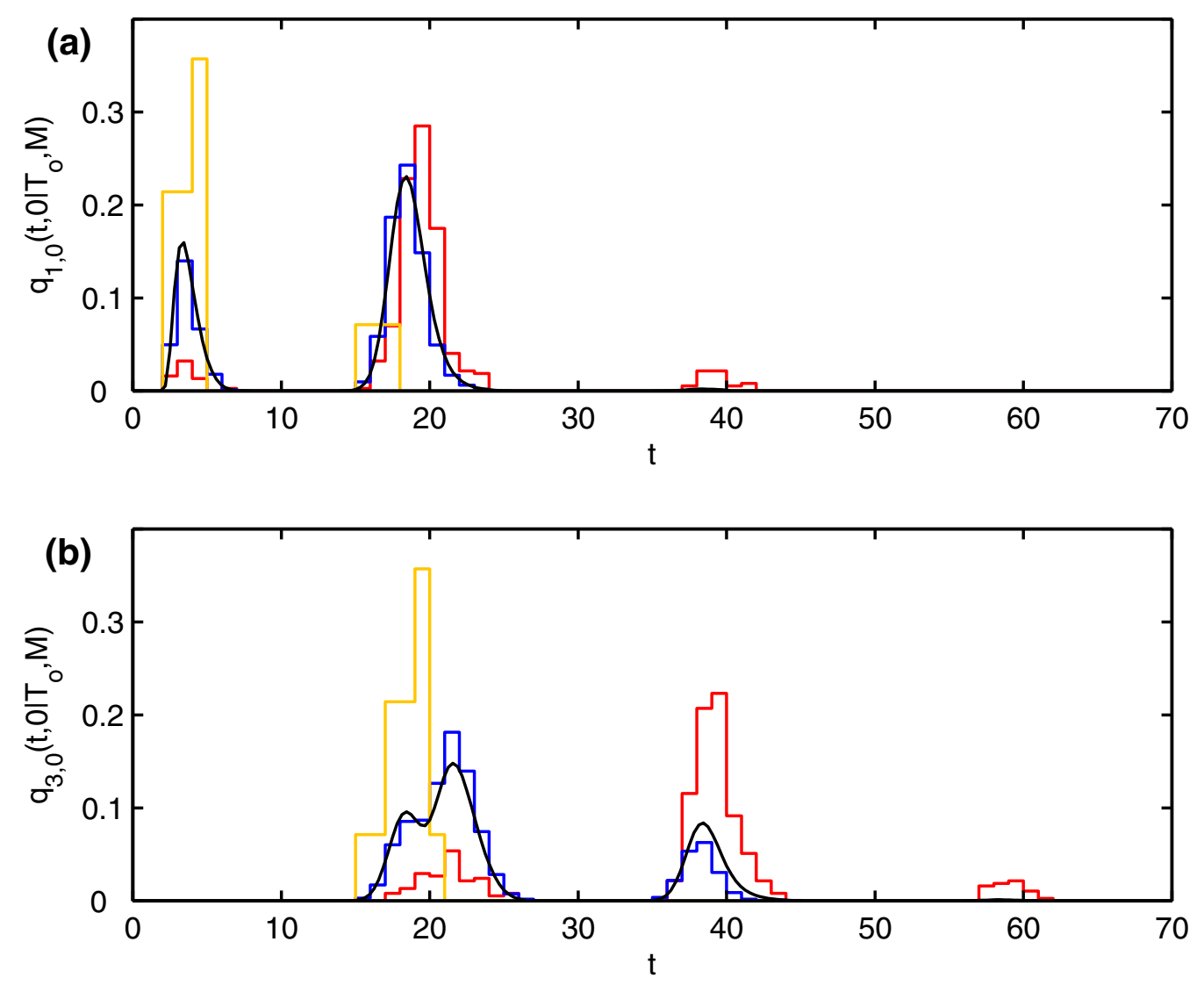

Figure 4.7: Joint spike time densities for (a) the first and (b) the third spike in a train. Colored histograms are count-conditional densities $q_{k, 0}\left(t, 0 \mid T_{o}, M\right)$ for spike counts $M=5$ (red), $M=8$ (blue), and $M=12$ (yellow). The black line is the plain jSTD $q_{k, 0}(t, 0)$. The expected spike count is $\langle M\rangle_{T_{o}}=7.8$ for $T_{o}=100$. All probabilities shown vanish for $t>60$.

corresponding count-conditional density

$q_{j+k, j}\left(t, s \mid T_{o}, M\right)=\operatorname{Prob}\left\{\begin{array}{l}\text { In train } \mathbf{t}, \text { the } j+k^{\text {th }} \text { spike occurs at } t \text { and the } \\ j^{\text {th }} \text { at } s, \text { provided } M \text { spikes occur in }\left(0, T_{o}\right] .\end{array}\right\}$.

Fig. 4.7 gives some examples of this density obtained from simulated spike trains compared to the plain joint spike-time density; for simplicity, $j=0$ and $s=0$ in the figure, so that $q_{k, 0}(t, 0)=q_{k}(t)$ could be exploited. The difference between plain and count-conditional joint spike-time density is striking and it is obviously impossible to approximate the latter by the former. The reason for this is that the requirement of a particular spike count $M$ imposes a global constraint on the spike train and thus destroys the Markov property: if the first interspike interval were very close to $T_{o}$, all following intervals had to be very short. Thus, the count-conditional (joint) spike time densities cannot be obtained via a Markov chain approach on principal grounds. 
The only solution to this conundrum are approximations, which differ for the constrained and unconstrained case. For the former, exact results are available in the limit of infinite observation time, while for the latter a reliable approximation to the power spectral density at the stimulus harmonics is obtained in Section 4.6.2. An approximation to the full spectrum is given for completeness in Section 4.6.3.

Before deriving expressions for the spectra, a crucial difference between the spectra evoked by constrained and unconstrained stimulation deserves comment. As indicated by the spike intensity [Fig. 4.3(b)], constrained stimulation does not elicit a periodic firing pattern (in a stochastic sense). Therefore, the spectrum will be continuous and bounded even for infinite observation time (Priestley 1996). This justifies the study of spectra in the limit of infinite observation time in this case. In response to unconstrained stimulation, though, the neuron fires periodically, as again indicated by the spike intensity, see Fig. 4.6. In consequence, the spectra possess a singular part, consisting of sharp peaks at multiples of the stimulus frequency. For $T_{o} \rightarrow \infty$, these peaks diverge, whence only the time-limited power spectral density is meaningful in this regime.

\subsubsection{PSD for constrained stimulation}

The power spectral density of the spike train elicited by constrained stimulation is quite easily obtained due to the renewal properties of the spike train. A comprehensive treatment with a view to neuroscience applications is given by Franklin and Bair (1995), albeit for constant stimuli; see also Plesser and Tanaka (1997). As pointed out above, exact results are available only for infinite $T_{o}$. Approximations for finite time were tested, but found to be reliable only in the presence of very large noise, when the spectrum reduces to nothing but background.

The spectrum is first derived for a fixed number of spikes, starting from Eq. (4.41), before taking the limit to infinity. For constrained stimulation, the joint spike time distribution simplifies to

$$
q_{j+k, j}(t, s)=q_{k}(t-s) q_{j}(s)
$$

with the results from Section 4.3. Therefore, the average over the exponential in Eq. (4.41) becomes

$$
\left\langle\mathrm{e}^{-i \omega\left(t_{j+k}-t_{j}\right)}\right\rangle=\int_{0}^{\infty} \int_{0}^{\infty} \mathrm{e}^{-i \omega(t-s)} q_{k}(t-s) q_{j}(s) \mathrm{d} t \mathrm{~d} s
$$

which upon the substitution $u=t-s$ simplifies to

$$
\left\langle\mathrm{e}^{-i \omega\left(t_{j+k}-t_{j}\right)}\right\rangle=\int_{0}^{\infty} q_{j}(s) \mathrm{d} s \int_{0}^{\infty} q_{k}(u) \mathrm{e}^{-i \omega u} \mathrm{~d} u=\bar{\rho}^{k}(i \omega) .
$$



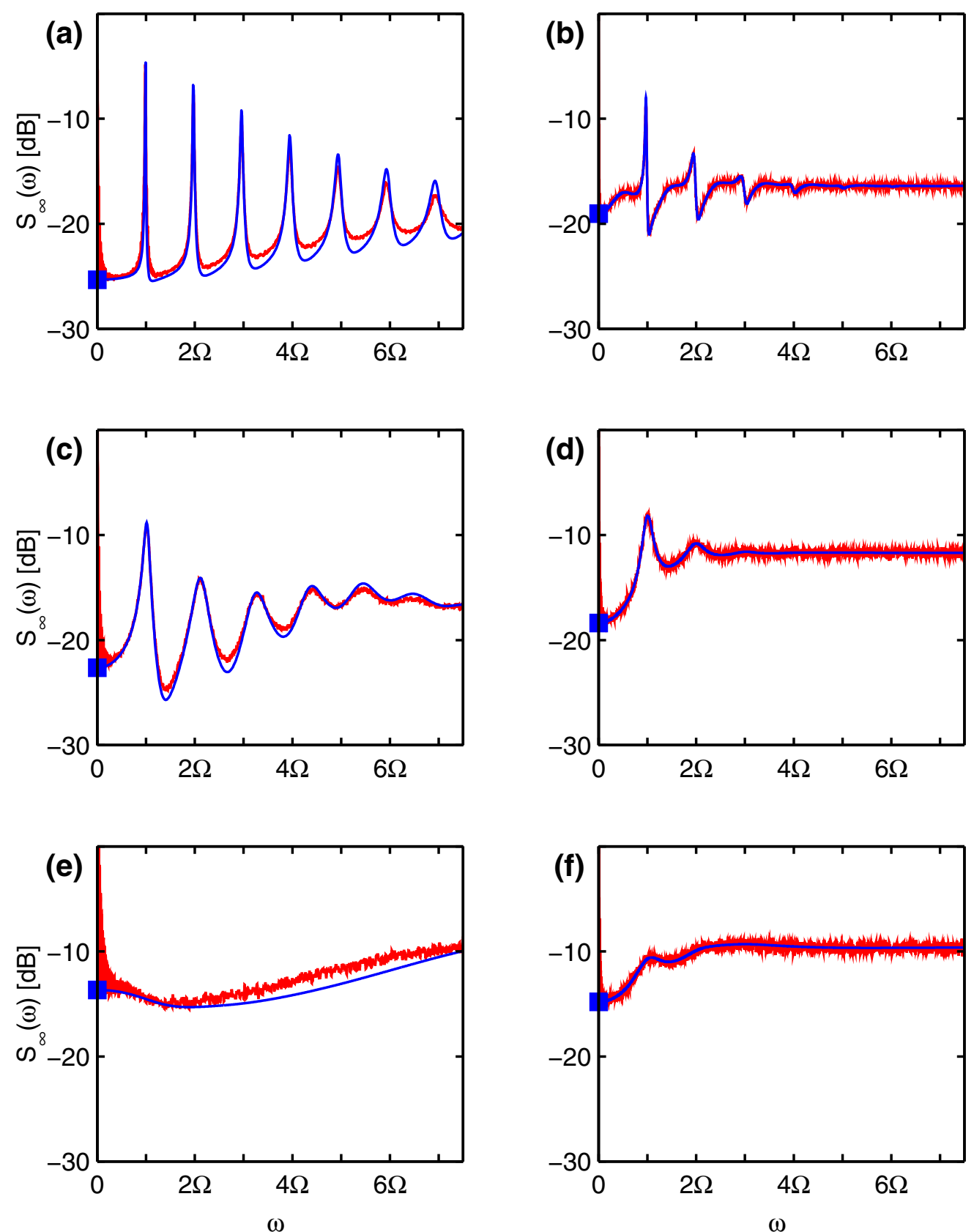

Figure 4.8: Power spectral density for constrained stimulation with sinusoidal stimuli. Renewal theory results are in blue, and spectra from simulated trains of $10^{5}$ spikes for $T_{o}=1000$ in red. Blue squares mark the limit $S_{\infty}(\omega \rightarrow 0)$ from Eq. (4.49). The abscissa is in units of the nominal stimulus frequency $\Omega$ and spectral power is in $\mathrm{dB}$. Stimulus frequencies were $\Omega=0.1$ with noise levels $\sigma=0.01,0.05,0.2$ (left, top to bottom), and $\Omega=0.5$ with $\sigma=0.05,0.15,0.3$ (right, top to bottom); other parameters: $\mu=0.9, q=0.1$, and $v_{R}=0$. 
Equation (4.10) has been used in the last step. $\bar{\rho}(i \omega)$ is the Laplace transform of the ISI density. It is equal to the Fourier transform $\tilde{\rho}(\omega) \equiv \bar{\rho}(i \omega)$ since $\rho(t)$ vanishes for negative values of the argument. Integrating over the entire positive real axis above is justified, since the result will be considered only in the limit $T_{o} \rightarrow \infty$. Inserting into Eq. (4.41) yields

$$
S_{T_{o}, M}(\omega)=\frac{M}{\pi T_{o}}\left[1+2 \operatorname{Re} \frac{\tilde{\rho}(\omega)}{1-\tilde{\rho}(\omega)}+\frac{2}{M} \operatorname{Re} \frac{\tilde{\rho}(\omega)\left(\tilde{\rho}(\omega)^{M}-1\right)}{(\tilde{\rho}(\omega)-1)^{2}}\right] .
$$

Now one has for the expected spike count of a renewal process at large times that $\langle M\rangle_{T_{o}} \rightarrow T_{o} /\langle\tau\rangle$ (Feller 1971) so that

$$
S_{\infty}(\omega)=\lim _{T_{o} \rightarrow \infty} S_{T_{o}, M}(\omega)=\frac{1}{\pi\langle\tau\rangle}\left[1+2 \operatorname{Re} \frac{\tilde{\rho}(\omega)}{1-\tilde{\rho}(\omega)}\right] .
$$

The spectrum as defined by Eq. (4.39) diverges at the origin, but Eq. (4.11b) yields

$$
\lim _{\omega \rightarrow 0+} S_{\infty}(\omega)=\frac{C_{\mathrm{v}}^{2}}{\pi\langle\tau\rangle}
$$

Fig. 4.8 compares the result of Eq. (4.48) to power spectra obtained from simulated spike trains (Appendix B). The agreement is excellent, and the result invites a few comments:

- The first term in brackets in Eq. (4.48) is the spectrum of a Poisson process with mean interspike interval $\langle\tau\rangle$. The spectrum of the modulated process converges to this Poisson spectrum for large frequencies.

- The spectral peaks are strongly smeared even for moderate noise, and for strong noise virtually no trace of the stimulus remains visible in the PSD.

- Since the spike train is not a periodic process under constrained stimulation, the peaks of the spectrum are shifted away from the harmonics of the stimulus frequency, complicating the evaluation of the signal-to-noise ratio, see Section 4.7 .

- The dip in the spectra at low frequencies is caused by the refractoriness of the neuron (Franklin and Bair 1995) and has been observed in experimental data (Bair et al. 1994).

- The divergence of Eq. (4.39) at $\omega=0$ leads to artefacts in the spectra obtained from simulated trains. The spectra obtained by renewal process analysis nicely converge to the limiting value of Eq. (4.49). 

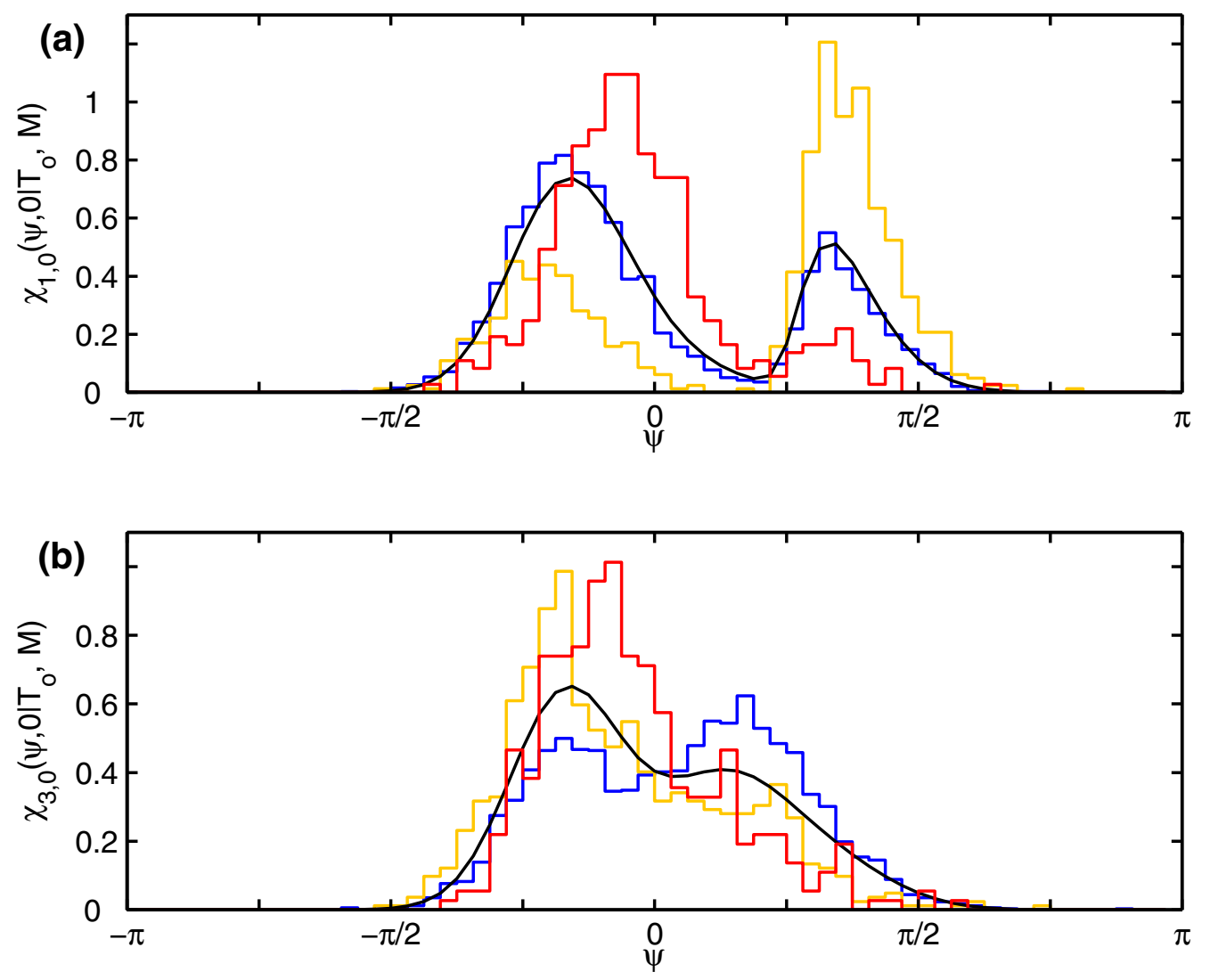

Figure 4.9: Joint spike phase densities for (a) the first and (b) the third spike in a train. Colored histograms are count-conditional densities $\chi_{k, 0}\left(\psi, 0 \mid T_{o}, M\right)$ for spike counts $M=5$ (red), $M=8$ (blue), and $M=12$ (yellow). The black line is the plain jSPD $\chi_{k, 0}(\psi, 0)$. All parameters are identical to Fig. 4.7.

\subsubsection{PSD at harmonics of unconstrained stimuli}

For a sinusoidal stimulus of frequency $\Omega$, the spike-count-conditional spectrum at the harmonics reduces to

$$
S_{T_{o}, M}(n \Omega)=\frac{M}{\pi T_{o}}\left[1+2 \operatorname{Re} h_{M}(n \Omega)\right]
$$

with

$$
h_{M}(n \Omega)=\frac{1}{M} \sum_{j=1}^{M-1} \sum_{k=1}^{M-j}\left\langle\mathrm{e}^{-i n\left(\psi_{j+k}-\psi_{j}\right)}\right\rangle
$$

and the spike phases $\psi_{j}$ as defined by Eq. (4.16). This average may be evaluated within the framework of the Markov chain in phase, offering essential advantages. First of all, the Markov chain in phase is asymptotically stable and the stationary density $\chi^{(s)}$ easily obtained as shown above. The spectrum 
can thus be computed in the stationary state. Second, as demonstrated by a comparison of Figs. 4.9 and 4.7, the count-conditional joint phase density

$$
\chi_{j+k, j}\left(\psi, \phi \mid T_{o}, M\right)=\operatorname{Prob}\left\{\begin{array}{l}
\text { The } j+k^{\text {th }} \text { spike occurs at } \psi \text { and the } j^{\text {th }} \\
\text { at } \phi, \text { provided } M \text { spikes occur in }\left(0, T_{o}\right] .
\end{array}\right\}
$$

is approximated better by its "plain" counterpart $\chi_{j+k, j}(\psi, \phi)$ than the corresponding spike time densities. The reason for this is that the global constraint on the spike count primarily alters the distribution of spikes between stimulus periods, but affects their distribution within periods but little. Plotting is facilited by $\chi_{k, 0}\left(\psi, 0 \mid T_{o}, M\right)=\chi_{k}(\psi)$.

Exploiting that $\chi_{j+k, j}(\psi, \phi)=\chi_{k}(\psi \mid \phi) \chi^{(s)}(\phi)$ in the stationary state, one may thus write to a good degree of approximation

$$
\left\langle\mathrm{e}^{-i n\left(\psi_{j+k}-\psi_{j}\right)}\right\rangle=\int_{0}^{2 \pi} \int_{0}^{2 \pi} \mathrm{e}^{-i n(\psi-\phi)} \chi_{k}(\psi \mid \phi) \chi^{(s)}(\phi) \mathrm{d} \phi \mathrm{d} \psi
$$

With an eye on numerical evaluation, the remainder of the derivation is given for the discrete Markov chain; the generalization to the continuous-phase Markov process should pose no principal difficulties. The discrete equivalent to Eq. (4.53) is

$$
\left\langle\mathrm{e}^{-i n\left(\psi_{j+k}-\psi_{j}\right)}\right\rangle=\hat{\mathbf{a}}(n)^{\operatorname{tr}} \cdot \mathbf{T}^{k} \cdot \hat{\mathbf{b}}(n)
$$

with vectors $(\Delta \psi=2 \pi / L$ is the phase discretization)

$$
\begin{gathered}
\hat{\mathbf{a}}(n)=\left(1, \mathrm{e}^{-i n \Delta \psi}, \mathrm{e}^{-2 i n \Delta \psi}, \ldots, \mathrm{e}^{-(L-1) i n \Delta \psi}\right)^{\operatorname{tr}} \\
\hat{\mathbf{b}}(n)=\left(\boldsymbol{\chi}_{0}^{(s)}, \boldsymbol{\chi}_{1}^{(s)} \mathrm{e}^{i n \Delta \psi}, \ldots, \boldsymbol{\chi}_{L-1}^{(s)} \mathrm{e}^{(L-1) i n \Delta \psi}\right)^{\operatorname{tr}} .
\end{gathered}
$$

Upon inserting Eq. (4.54) into Eq. (4.51), observe that $\left\langle e^{i n\left(\psi_{j+k}-\psi_{j}\right)}\right\rangle$ depends only on the "distance" $k$ of the spikes, but not on their "position" $j$ due to stationarity. Hence the outer summation over $j$ may be performed to obtain

$$
h_{M}(n \Omega)=\hat{\mathbf{a}}(n)^{\operatorname{tr}}\left[\frac{1}{M} \sum_{j=1}^{M-1}(M-j) \mathbf{T}^{j}\right] \hat{\mathbf{b}}(n) .
$$

Diagonalizing $\mathbf{T}$ leads to

$$
h_{M}(n \Omega)=\mathbf{a}(n)^{\operatorname{tr}} \cdot \mathbf{S}^{(M)} \cdot \mathbf{b}(n)
$$

where $\mathbf{S}^{(M)}$ is a diagonal matrix with elements ${ }^{11}$

$$
\mathbf{S}_{m m}^{(M)}= \begin{cases}\frac{M-1}{2} & \text { for } m=1, \\ \frac{\lambda_{m}}{1-\lambda_{m}}+\frac{1}{M} \frac{\lambda_{m}\left(\lambda_{m}^{M}-1\right)}{\left(\lambda_{m}-1\right)^{2}} & \text { for } m>1 .\end{cases}
$$

\footnotetext{
${ }^{11}$ The corresponding Eq. (A5) of Plesser and Geisel (1999) contains a typographical error.
} 
The $\lambda_{m}$ are the ordered eigenvalues of $\mathbf{T}\left(\lambda_{1}=1>\left|\lambda_{2}\right| \geq \ldots \geq\left|\lambda_{L}\right|\right)$, and the vectors $\mathbf{a}(n)$ and $\mathbf{b}(n)$ are given by

$$
\mathbf{a}(n)=\mathbf{C}^{\operatorname{tr}} \hat{\mathbf{a}}(n), \quad \mathbf{b}(n)=\mathbf{C}^{-1} \hat{\mathbf{b}}(n)
$$

with the matrix decomposition

$$
\mathbf{T}=\mathbf{C} \cdot \mathbf{L} \cdot \mathbf{C}^{-1}, \quad \mathbf{L}=\operatorname{diag}\left(\lambda_{1}=1, \lambda_{2}, \ldots, \lambda_{L}\right)
$$

Note that the term for $m=1$ has no counterpart in the constrained case, while the terms for $m>1$ are very similar to the second and third terms of the power spectral density for the constrained case, cf. Eq. (4.47). Inserting Eq. (4.55) into Eq. (4.50) yields

$$
S_{T_{o}, M}(n \Omega)=\frac{M}{\pi T_{o}}\left[1+2 \operatorname{Re} \mathbf{a}^{\operatorname{tr}}(n) \mathbf{S}^{(M)} \mathbf{b}(n)\right] .
$$

The matrix element $\mathbf{S}_{11}^{(M)}$ is linear in the number $M$ of spikes, and, since $T_{o} / M \rightarrow\langle\tau\rangle$ asymptotically, this term gives rise to the singular spectral component that grows linearly with the length of the spike train. It is convenient to split the spectrum into its singular and bounded parts

$$
S_{T_{o}, M}(n \Omega)=\frac{M}{\pi T_{o}}[1+A(n, M)+(M-1) B(n)]
$$

with the bounded background

$$
A(n, M)=2 \operatorname{Re}\left[\mathbf{a}^{\operatorname{tr}}(n) \operatorname{diag}\left(0, \mathbf{S}_{22}^{(M)}, \ldots, \mathbf{S}_{L L}^{(M)}\right) \mathbf{b}(n)\right]
$$

and the singular periodic contribution

$$
B(n)=\operatorname{Re} \mathbf{a}_{1}(n) \mathbf{b}_{1}(n)=\left|\sum_{j=0}^{L-1} \chi_{j}^{(s)} \mathrm{e}^{i j n \Delta \psi}\right|^{2}
$$

The last equality follows directly from Eq. (4.57), because the first column of $\mathbf{C}$ is an eigenvector of $\mathbf{T}$ to eigenvalue $\lambda_{1}=1$ and thus equal to the stationary phase distribution $\chi^{(s)}$ up to a factor $c \neq 0$. The first row of $\mathbf{C}^{-1}$ as the corresponding right eigenvector has all elements equal to $1 / c$, yielding the result above. Note that $A(n, M)$ is bounded for $M \rightarrow \infty$, since $\left|\lambda_{m}\right|<1$ for $m>1$ from Section 4.4.2 so that

$$
\lim _{M \rightarrow \infty}\left|\mathbf{S}_{m m}^{(M)}\right|=\left|\frac{\lambda_{m}}{1-\lambda_{m}}\right|<\infty, \quad m>1 .
$$



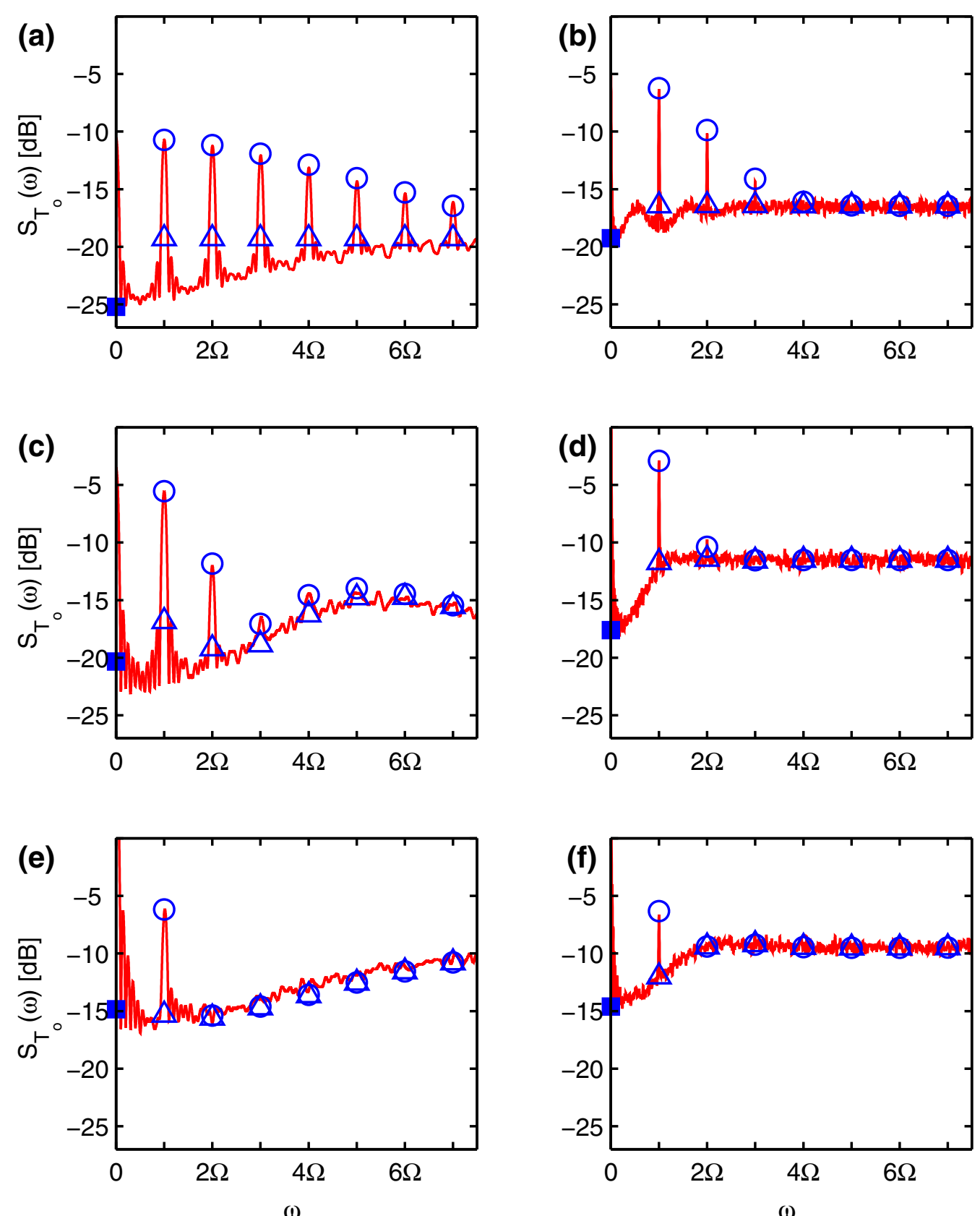

Figure 4.10: Power spectral density for unconstrained stimulation with the same sinusoidal stimuli used in Fig. 4.8 and observation time $T_{o}=200$. Results at harmonics from Markov chain approach in blue, and spectra from simulated trains in red. Circles mark the estimated spectrum $S_{T_{o}}$, triangles mark bounded part $\left[1+A\left(n, T_{o} /\langle\tau\rangle\right)\right] / \pi\langle\tau\rangle$, and the filled square the limit of the spectrum at the origin from Eq. (4.49). The abscissa is in units of the stimulus frequency $\Omega$ and spectral power is in $\mathrm{dB}$. 

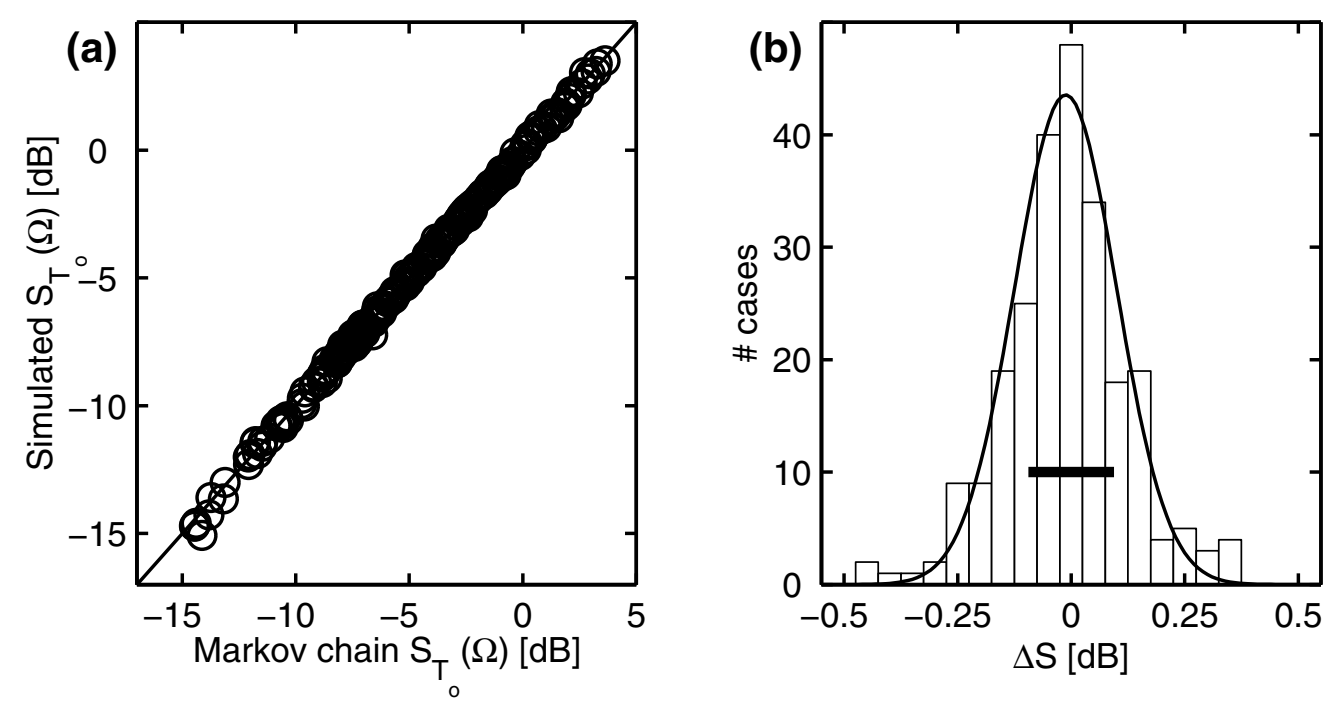

Figure 4.11: Power spectral density at stimulus frequency $S(\Omega)$ from Markov chain (Eq. 4.62) and simulated trains are in excellent agreement. (a) Scatter plot of results from 245 parameter sets with Markov chain result on the abscissa and simulation result on ordinate; error bars for simulation results are shorter than the symbol size. The correlation coefficient is $>0.999$. Observation time is $T_{o}=200$. (b) Distribution of difference $\Delta S$ between simulation and Markov chain results, and fitted Gaussian distribution with mean $\langle\Delta S\rangle=-0.01 \mathrm{~dB}$ and standard deviation $\sqrt{\left\langle\Delta S^{2}\right\rangle}=0.11 \mathrm{~dB}$. The thick black bar marks two standard errors of the mean of the simulation results.

In analogy to the constrained case, the spike count is now estimated as ${ }^{12}$ $M=T_{o} /\langle\tau\rangle \approx\langle M\rangle_{T_{o}}$ to obtain the time-limited PSD at the stimulus harmonics from Eq. (4.59)

$$
S_{T_{o}}(n \Omega) \approx \frac{1}{\pi\langle\tau\rangle}\left[1+A\left(n, \frac{T_{o}}{\langle\tau\rangle}\right)+\left(\frac{T_{o}}{\langle\tau\rangle}-1\right) B(n)\right]
$$

Figure 4.10 indicates excellent agreement between the power spectral density at the harmonics given by this approximation with spectra obtained from simulated spike trains via discrete Fourier transform.

To quantify the quality of the approximation, $S(\Omega)$ was computed for a large number of stimuli from Eq. (4.62) and from simulated trains of 50000 spikes. ${ }^{13}$ The results are shown in Fig. 4.11 and indicate near-perfect agreement. The standard deviation of the difference $\Delta S$ between simulation and Markov chain results is only $0.11 \mathrm{~dB}$, which is on the order of the statistical error of the simulation results, while the mean of $\Delta S$ is nearly zero $(-0.01 \mathrm{~dB})$.

\footnotetext{
${ }^{12}$ Plesser and Geisel (1999) used $M=\left\lfloor T_{o} /\langle\tau\rangle\right\rfloor$ to approximate the spike count averaging; the precise value of the fraction improves the approximation slightly.

${ }^{13}$ Stimulus parameters were an evenly spaced subset of those used in Fig. 5.17, p. 104.
} 

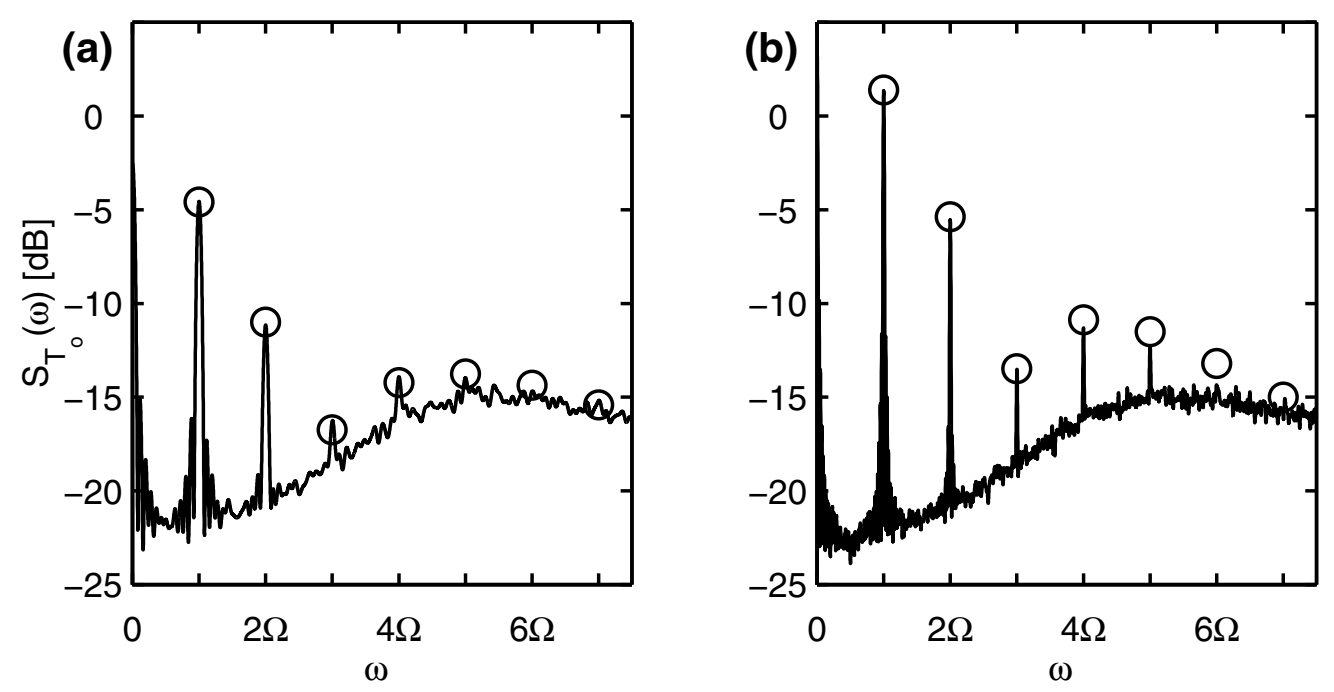

Figure 4.12: PSD at the stimulus harmonics increases with observation time for unconstrained stimulation, while the background is independent of $T_{o}$ : (a) $T_{o}=250$ and (b) $T_{o}=1000$. Filter ringing is reduced at larger observation times. The stimulus is the same as in Fig. 4.10(c). The abscissa is in units of the stimulus frequency $\Omega$ and spectral power in $\mathrm{dB}$.

The resulting spectra invite some comments:

- The first term of the power spectral density is again the Poisson train PSD.

- The stimulus is clearly discernible in the spectrum for unconstrained stimulation even for those stimuli where it has become invisible in the constrained case, compare Fig. 4.8.

- For long times $T_{o}$ and consequently large spike numbers $M$, the spectrum is dominated by the singular part $\sim M B(n)$, compare Fig. 4.12. It arises from the persistent eigenmode to eigenvalue $\lambda_{1}=1$, and represents the long-range correlations between spikes induced by the synchronization of the spike train to the stimulus. One might call it the PSD of the stationary phase density $\chi^{(s)}$, see Eq. (4.61). The peaks at the harmonics thus decay the faster, the wider the phase density. For perfect phaselocking, i.e. $\chi^{(s)}(\psi)=\delta\left(\psi-\psi_{0}\right)$, the peaks would not decay at all.

- Since the Markov chain in phase does not describe the distribution of spikes across periods, it yields no information about the noise background of the spectrum. In particular, the continuous part of the spectrum $\sim[1+A(n, M)]$ (triangles in Fig. 4.10) does not reliably estimate the noise level unless the neuron rarely skips a stimulus period without firing. 
- The dip of the spectrum at lowest frequencies is due to refractory effects as for the constrained stimulation. Since refractoriness should depend little on stimulation details, the limiting value $S_{\infty}(\omega \rightarrow 0)$ given by Eq. (4.49) applies to the spectra of unconstrained spike trains as well, as shown in Fig. 4.10.

- The derivation above applies to non-sinusoidal periodic stimuli if $\Omega$ is replaced by the pseudo-frequency $\hat{\Omega}$. The resulting spectral values at multiples of $\hat{\Omega}$ will usually be of little interest.

- The full spectra displayed in Fig. 4.10 for comparison show noticeable filter ringing particularly for low stimulus frequencies. This is a consequence of the rectangular window $\left[0, T_{o}\right]$ imposed in the definition of $S_{T_{o}}$. It is thus an artefact of the definition, not a numerical one. Ringing might be reduced by using windows with softer flanks (Press et al. 1992), but spectra would no longer be in accordance with the definition of $S_{T_{o}}$, cf. Eq. (4.39). Since the evaluation of the power spectral densities at the harmonics in closed form would be severely complicated if, e.g., a Hanning window were incorporated in the definition, only the rectangular window is used here. Errors at the spectral peaks are negligible provided that the observation time $T_{o} \gg 2 \pi / \Omega$. This condition is met throughout.

\subsubsection{Full PSD for unconstrained stimulation}

The complete power spectral density for periodic stimuli may be approximated using the Markov chain in time introduced in Section 4.4.3, although results are not as compact as those obtained in the two preceeding cases. Empirically, the best available estimate of the count-conditional joint spike time density is

$$
q_{j+k, j}\left(t, s \mid T_{o}, M\right) \approx \hat{q}_{k}(t-s \mid s) \hat{q}_{j}\left(s \mid t_{0}=0\right)
$$

with the re-normalized conditional spike time density

$$
\hat{q}_{k}(t-s \mid s)=q_{k}(t-s \mid s) / \int_{s}^{T_{o}} q_{k}(t-s \mid s) \mathrm{d} t .
$$

The average in Eq. (4.41) may thus be written as

$$
\left\langle\mathrm{e}^{i \omega\left(t_{j+k}-t_{j}\right)}\right\rangle=\int_{0}^{T_{o}} \int_{0}^{T_{o}} \mathrm{e}^{i \omega(t-s)} \hat{q}_{k}(t-s \mid s) \hat{q}_{j}(s \mid 0) \mathrm{d} t \mathrm{~d} s .
$$

Assume now as in Section 4.4.3 that time is discretized as $\Delta t=T / L$ with $T=2 \pi / \hat{\Omega}$ and that the time transition matrix $\mathbf{Q}$ is of size $N \times N$ with $T_{o}=N \Delta t$. The renormalized conditional spike time density $\hat{\mathbf{Q}}_{k}(t \mid s)$ is then 

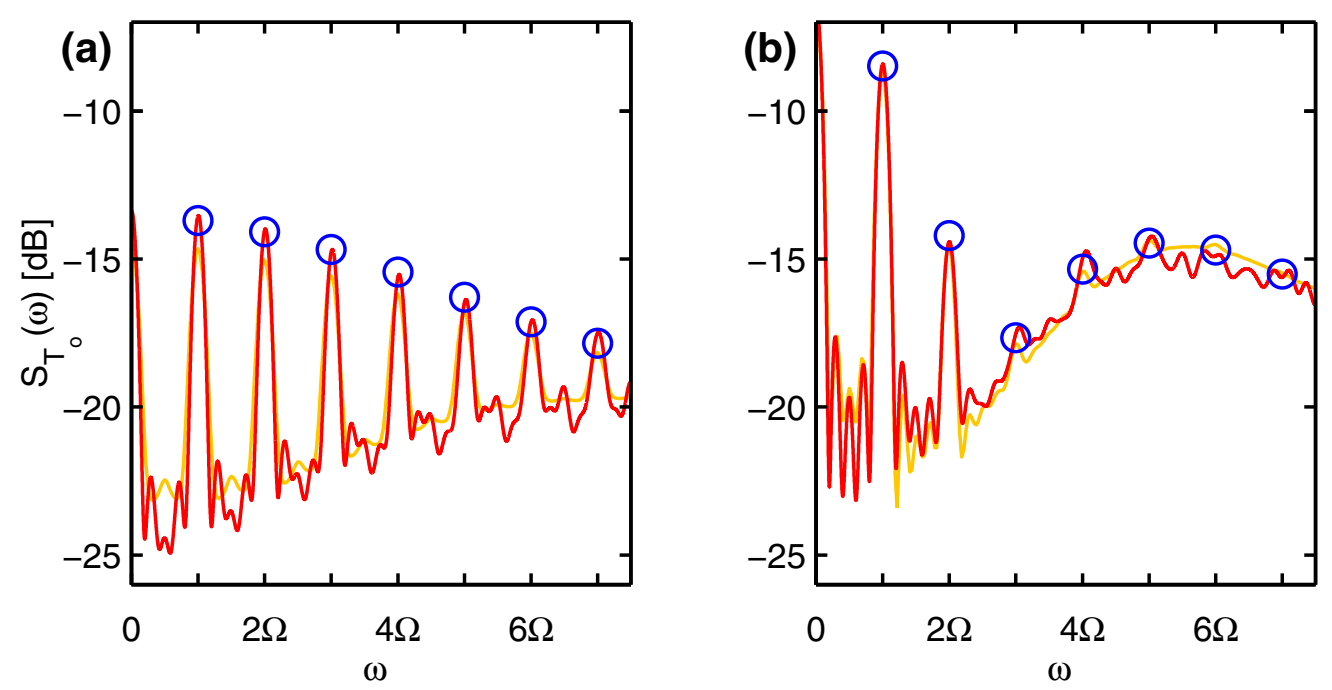

Figure 4.13: The full power spectral density may be approximated by means of the Markov chain in time: PSD for unconstrained stimulation with the same sinusoidal stimuli as in Fig. 4.10(a) in (a) and Fig. 4.10(c) in (b). The spectrum from simulated spike trains is in red, the approximated spectrum of Eq. (4.66) in yellow and the blue circle give the phase matrix result at the harmonics.

given by the matrices $\hat{\mathbf{Q}}^{k}$, which are obtained from $\mathbf{Q}^{k}$ by renormalizing each column to a unit sum. One thus obtains

$$
\left\langle\mathrm{e}^{i \omega\left(t_{j+k}-t_{j}\right)}\right\rangle=\sum_{\ell=1}^{N} \sum_{n=\ell+1}^{N} \mathrm{e}^{i(n-\ell) \omega \Delta t} \hat{\mathbf{Q}}_{n \ell}^{k} \hat{\mathbf{Q}}_{\ell 1}^{j},
$$

where the inner summation starts from $\ell+1$ since no two spikes can occur at the same time. This double sum cannot be simplified as in the previous section, since no stationary state exists for the spike time Markov chain. The spike-count-conditional PSD is therefore from Eq. (4.41)

$$
S_{T_{o}, M}(\omega)=\frac{M}{\pi T_{o}}+\frac{2}{\pi T_{o}} \operatorname{Re} \sum_{\ell=1}^{N} \mathrm{e}^{-i \ell \omega \Delta t}\left\{\sum_{j=1}^{M-1}\left[\sum_{n=\ell+1}^{L} \mathrm{e}^{i n \omega \Delta t}\left(\sum_{k=1}^{M-j} \hat{\mathbf{Q}}_{n \ell}^{k}\right)\right] \hat{\mathbf{Q}}_{\ell 1}^{j}\right\} .
$$

The sums have been re-ordered to reflect the optimal arrangement for evaluation by computer; an implementation is given in Plesser (1999). As the sums above have to be evaluated explicitly, it is not possible to use fractional values for the spike count $M$ as in the preceeding cases. Instead, one has to resort to $M=\left\lfloor T_{o} /\langle\tau\rangle\right\rfloor$ as summation limit. The example given in Fig. 4.13 indicates fair agreement with simulated spectra. For small spike counts in the observation time, the deviations at the stimulus harmonics become considerable; the phase matrix results are much closer to the true spectrum in this case. 
The computation of the full spectrum requires prohibitively more computer time and memory than the phase matrix approach, since all powers of the matrix $\mathbf{Q}$ up to some $M=\left\lfloor T_{o} /\langle\tau\rangle\right\rfloor$ have to be computed and stored. Even with the power of workstations available today, the use of the full spectrum is limited. For some technical considerations such as the choice of discretization parameters, see Plesser (1999).

\subsection{Signal-to-noise ratio}

The signal-to-noise ratio SNR is a statistic with little tradition in the neurosciences, but deeply engrained in the theory of stochastic resonance. It measures the signal power contained in the spike train at the frequency $\Omega$ of the driving sinusoidal stimulus relative to the "background activity" in the train. As explained above, the signal-to-noise ratio will be measured for a finite observation time $T_{o}$, except for constrained stimulation, where $T_{o}=\infty$ is justified.

The signal power $S_{T_{o}}(\Omega)$ at the stimulus frequency is easily obtained from Eq. (4.62) under unconstrained stimulation. For constrained stimulation a complication arises, since the firing pattern is non-periodic, whence the spectral peaks are shifted away from $\Omega$. Thus, the signal power is taken to be the maximum of the spectrum in a window around the stimulus frequency

$$
S_{\infty}^{\prime}(\Omega)=\max _{0.9 \Omega<\omega<1.1 \Omega} S_{\infty}(\Omega)
$$

and

$$
\Omega_{\max }=\underset{0.9 \Omega<\omega<1.1 \Omega}{\arg \max } S_{\infty}(\Omega)
$$

is the location of the spectral peak. If $S(\omega)$ has no relative maximum inside the interval, $S_{\infty}^{\prime}(\Omega)$ is undefined.

The noise background is even more difficult to determine. If the spectral peaks are strongly smeared, as in Fig. 4.8(c), it is hard to decide where the signal ends and the noise begins. Furthermore, for the interesting case of unconstrained stimulation, the power spectral density off the stimulus harmonics can only be approximated, and even this at prohibitive computational cost. But the spectra quickly approach the white power spectrum

$$
S_{P}=1 / \pi\langle\tau\rangle
$$

of a Poisson process for larger frequencies, as a glance at Figs. 4.8 and 4.10 reveals. Since the mean ISI $\langle\tau\rangle$ is easily computed, cf. Table 4.1, it has been used by several authors to estimate the noise background (Plesser and Geisel 
1999; Plesser and Tanaka 1997; Stemmler 1996; Shimokawa et al. 1999a). The signal-noise-ratio is therefore defined as

$$
\begin{array}{ll}
\mathrm{SNR}_{\infty}=S_{\infty}^{\prime}(\Omega) / S_{P} & \text { for constrained stimuli, } \\
\mathrm{SNR}_{T_{o}}=S_{T_{o}}(\Omega) / S_{P} & \text { for unconstrained stimuli. }
\end{array}
$$

The subscripts $\infty$ and $T_{o}$ will usually be dropped for brevity.

Basing the SNR on the Poisson spectrum may be interpreted in a more elementary way: The SNR measures how well the periodically modulated spike train contrasts with a homogeneous Poisson train background of comparable intensity. 


\section{Chapter 5}

\section{Noise Aided Signal Processing}

With all required tools at hand, the stage is set for the task proper: the analysis of signal processing properties of the neuronal spike generator. In particular, this chapter aims to clarify the effect of Gaussian white noise on the transformation of a continuous sinusoidal input current into a spike train. With a view to the all-or-nothing nature of spike trains, this may be interpreted as an analog-to-digital conversion. As it has been suggested that the brain achieves its tremendous capabilities at minute power consumption through an intricate combination of analog signal processing within neurons and digital signaling between them, this A/D conversion is crucial to the nervous system (Sarpeshkar 1998). It is shown here that the encoding of subthreshold membrane potential fluctuations into spike trains benefits from noise through stochastic resonance.

The encoding of constrained stimuli into spike trains will be treated in Section 5.1. It will be shown that the Ornstein-Uhlenbeck neuron exhibits stochastic resonance at small noise amplitudes in this regime due to frequency matching: the signal-to-noise ratio is maximized when interspike intervals coincide with multiples of the effective stimulus period. Since this effective period will be similar to the nominal stimulus period only for a small range of initial stimulus phases, stochastic resonance occurs but under peculiar circumstances. In particular, it is demonstrated that stochastic resonance disappears in this artificial regime if the initial phase $\phi_{0}$ adapts to the noise applied.

The biologically relevant unconstrained stimulation is then studied in the remainder of this chapter. The dependence of essential response properties such as the interspike-interval and spike phase densities, and of the spike intensity on stimulus parameters are discussed in detail. They are shown to be in good agreement with data obtained from sensory afferent neurons in monkeys and cats. The Markov chain method opens the way to analyze the complex firing patterns observed in these neurons without resorting to analog or digital simulations. In particular, the cross-over from the transmission of pulse packets to regular firing of individual spikes, as is found in cold receptors with increasing temperature, is reproduced well by the model. 


\begin{tabular}{lllllll}
\hline base current & $\mu=0.9$ & frequency & period & \multicolumn{2}{c}{ noise amplitude $\sigma$} \\
stim. amplitude & $q=0.1$ & $\Omega=0.1 \pi$ & $T=20$ & 0.01 & 0.053 & 0.25 \\
reset potential & $v_{R}=0$ & $\Omega=0.33 \pi$ & $T=6$ & 0.03 & 0.064 & 0.25 \\
initial phase & $\phi_{0}=0$ & $\Omega=0.5 \pi$ & $T=4$ & 0.05 & 0.074 & 0.25 \\
\hline
\end{tabular}

Table 5.1: Parameters of the standard stimulus set used for illustrative examples. Base current, stimulus amplitude, reset potential and initial phase are the same for all nine examples, which differ in frequency and noise. Because of the low-pass behavior of the OrnsteinUhlenbeck neuron, stronger noise is required at higher frequencies to evoke responses.

The key result of this thesis is established in Section 5.3: signal processing in the Ornstein-Uhlenbeck neuron is fostered by noise through stochastic double resonance (SDR). Besides classic stochastic resonance, i.e. the maximization of the signal-to-noise ratio of the output at a particular input noise amplitude, the neuron also benefits from bona fide stochastic resonance. The latter permits the neuron to achieve a particularly high output signal-to-noise ratio at the resonance frequency. Noise thus furnishes the neuron with bandpass properties. The origins of this effect are elucidated in detail. The resonance in noise is traced to a compromise between signal quality (vector strength) and signal intensity (firing rate), while the resonance in stimulus frequency is shown to arise through a matching of timescales. The dependence of the response properties of the neuron on base current, stimulus amplitude and reset potential are studied quantitatively. Stochastic double resonance is shown to prevail across a wide range of these parameters. The optimal signal-to-noise ratio is found to depend on scaled model parameters in a straightforward fashion. In particular, $\sigma_{r}^{\text {opt }} \approx 2 / 3$ is identified as optimal input noise amplitude independent of all other stimulus parameters. A detailed analysis based on the Arrhenius\&Current model reveals the mechanisms giving rise to the observed parameter dependence.

\subsection{Constrained stimulation}

When a neuron is driven by a constrained periodic stimulus, this stimulus is reset to some initial phase $\phi_{0}$ after each spike. The effective stimulus delivered to the neuron will thus reflect the periodicity of the nominal stimulus - the one that were delivered without reset - only if spikes occur preferentially at a phase near $\phi_{0}$, cf. Fig. 4.2 , p. 52. Therefore, the spike train generated by the neuron will properly encode the nominal stimulus only if stimulus and reset are properly matched. 


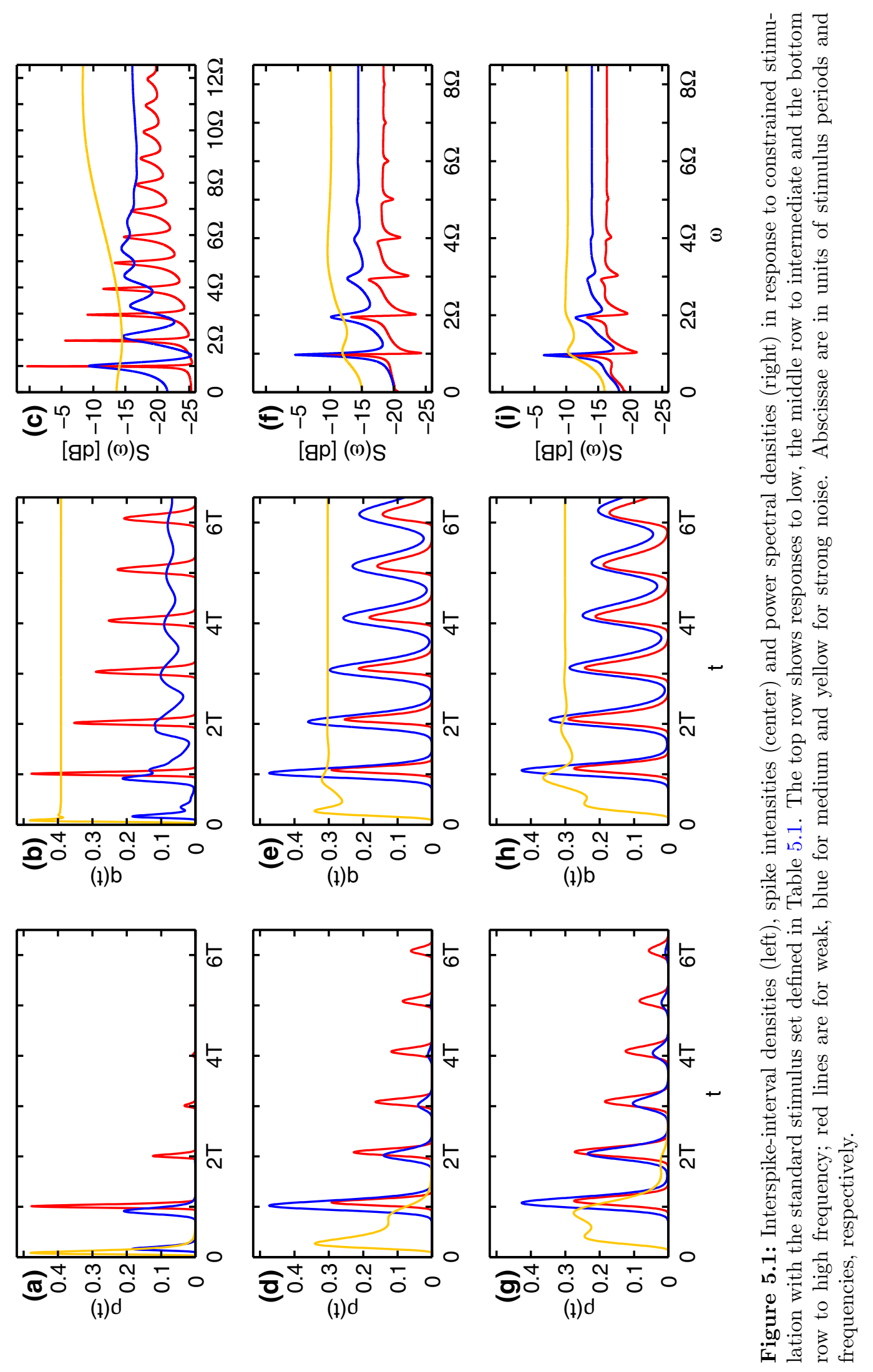


For sinusoidal stimuli of the form

$$
I(t)=\mu+q \cos \left(\Omega t+\phi_{0}\right)
$$

as studied here, nearly periodic responses are obtained in the vicinity of $\phi_{0}=0$. This is the initial phase chosen in all previous work on the constrained regime (Plesser and Tanaka 1997; Shimokawa et al. 1999a; Bulsara et al. 1996). For illustrative purposes, a standard set of stimuli comprising low, medium and high frequencies, each combined with three different noise amplitudes will be used throughout this chapter. The parameter values are given in Table 5.1.

Figure 5.1 shows the response of the Ornstein-Uhlenbeck neuron to this standard stimulus set in terms of the interspike-interval density, spike intensity, and power spectrum. The ISI density $\rho(t)$ varies strongly with noise. For small noise amplitudes $\sigma$ (red lines in the figure), it consists of a series of nearly Gaussian modes separated by the stimulus period $T$. These peaks are located close to multiples of the period only because of the particular choice of the initial phase $\phi_{0}=0$; an example for $\phi_{0}=\pi / 2$ is given in Fig. 5.2(b). With the possible exception of the first modes at high stimulus frequencies, the height of these Gaussian modes decays exponentially for large times. Since the peaks are narrow and close to multiples of $T$, the neuron fires regularly around multiples of the stimulus period, as shown by the spike intensity $q(t)$. As time proceeds, jitter accumulates over subsequent interspike intervals and the peaks of the spike intensity widen gradually. The spike intensity approaches its stationary value $1 /\langle\tau\rangle$ in the limit of long times even for low noise. The power spectral density has sharp peaks close to the nominal stimulus frequency $\Omega$, and tends to a white Poisson spectrum for larger frequencies. The lowpass nature of the neural integrator is reflected by the fact that the peaks of all densities/intensities broaden with frequency. Since the supremum of the noise-free membrane potential (cf. Fig. 5.18, p. 106)

$$
v_{\infty}=\sup _{t \geq 0} v_{0}(t)=\mu+\frac{q}{\sqrt{1+\Omega^{2}}}
$$

decreases with increasing $\Omega$, stronger noise is required to make the neuron fire (at measurable rate) for high frequencies.

The strong noise limit (yellow in Fig. 5.1) is characterized by an ISI mode at short times which is not related to the stimulus period $T$, but reflects the refractory time of the neuron. ${ }^{1}$ For very large noise, this peak tends towards a gamma density regardless of stimulus frequency. Since the neuron fires essentially at random in this regime, the spike intensity $q(t)$ flattens almost instantly. For intermediate noise (blue lines), a cross-over occurs. At low frequencies in particular, clearly separated refractory and periodic modes of the

\footnotetext{
${ }^{1}$ This is the time required for the membrane potential to recover from the reset potential $v_{R}=0$ to within roughly one noise amplitude from the threshold $\Theta=1$.
} 

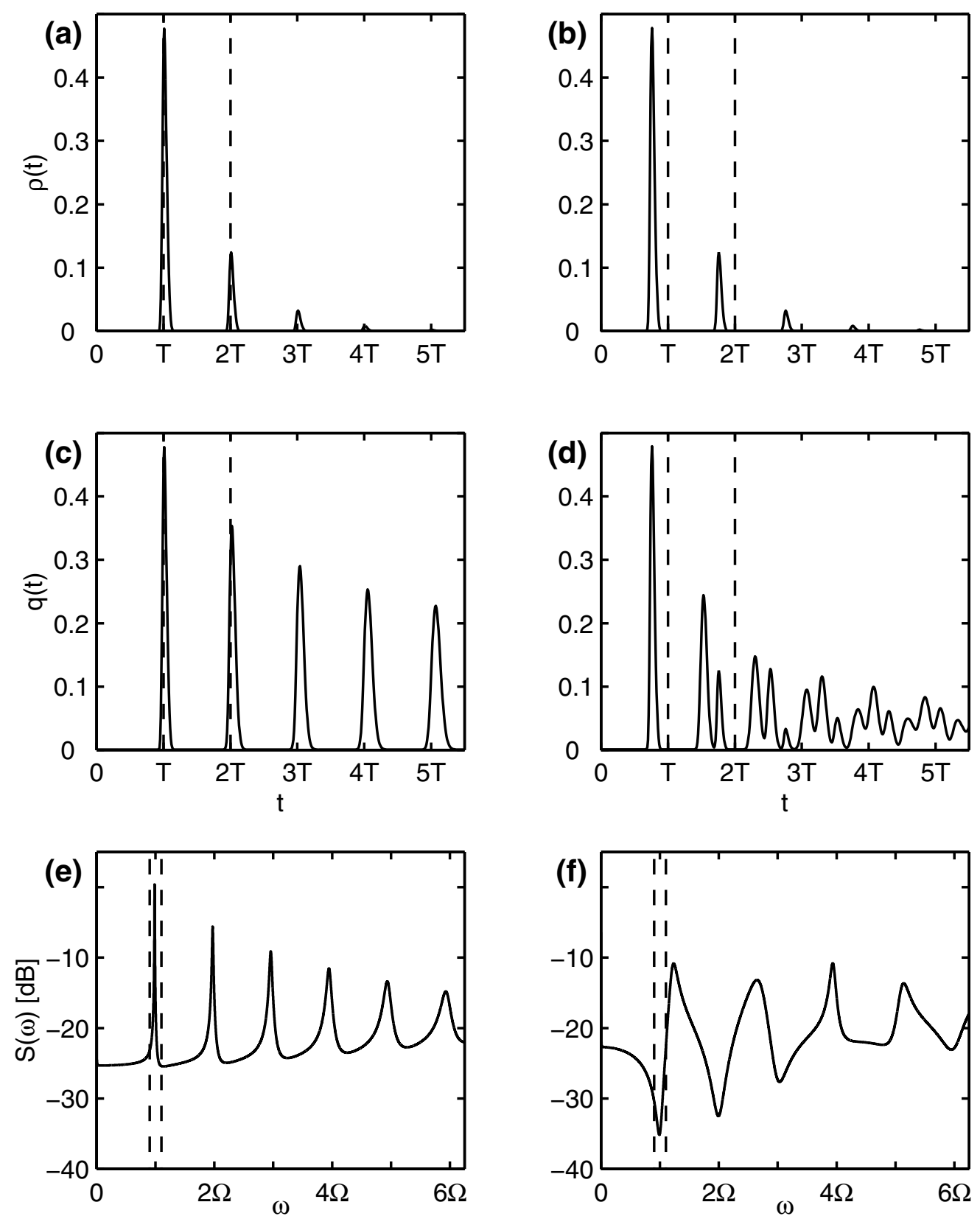

Figure 5.2: The response to constrained stimulation depends markedly on the initial phase $\phi_{0}$ : Interspike-interval densities (top), spike intensities (middle), and power spectral densities (bottom) in response to constrained stimulation as shown in Fig. 4.2. The left column is for $\phi_{0}=0$, the right one for $\phi_{0}=\pi / 2$. Dashed lines mark the first and second multiples of the stimulus period $T=20$ in (a) through (d), and the frequency window $(0.9 \Omega, 1.1 \Omega)$ used for signal power determination in (e) and (f), see Eq. (4.67). Resetting the stimulus to the "wrong" phase $\phi_{0}=\pi / 2$ prevents a synchronized response, so that the spectrum has no peak around the stimulus frequency $\Omega$. The stimulus is the weak noise, low frequency stimulus of Table 5.1. 


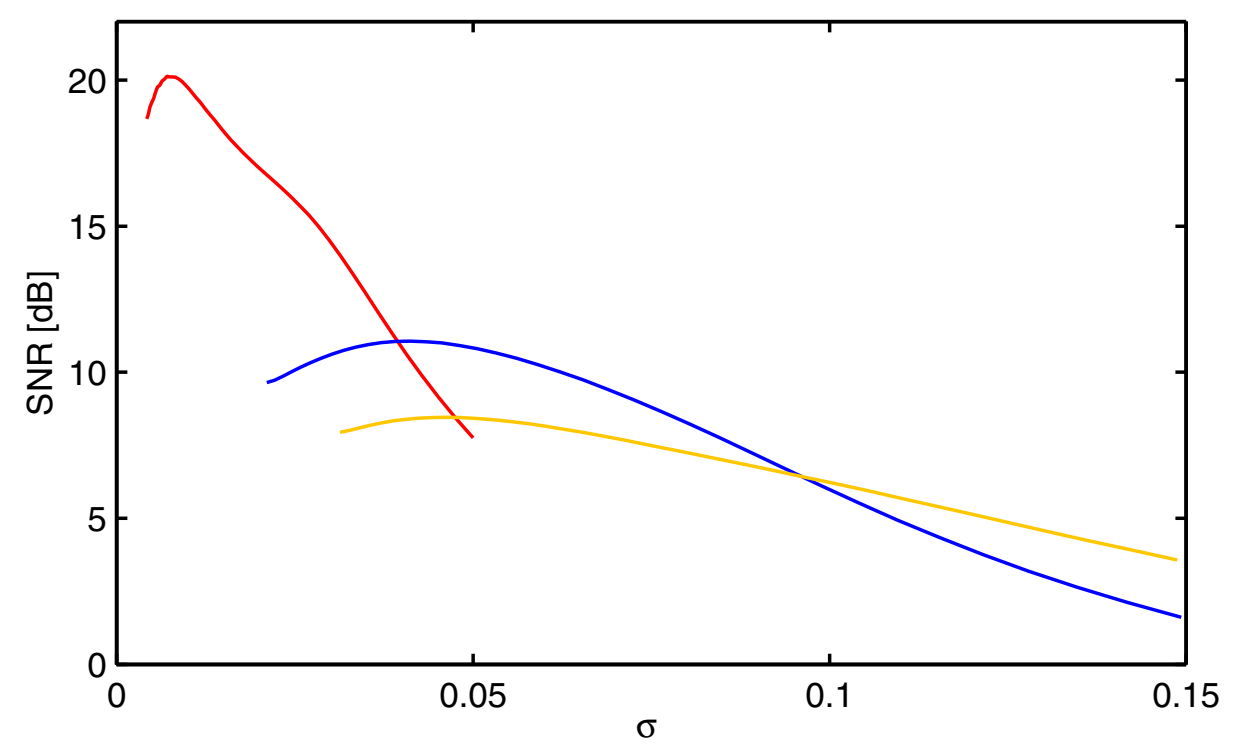

Figure 5.3: Stochastic resonance under constrained stimulation: the signal-to-noise ratio SNR peaks at a non-vanishing input noise amplitude $\sigma_{\max }$. This noise amplitude and the SNR attained depend strongly on the stimulus frequency: $\Omega=0.1 \pi$ (red), $\Omega=0.33 \pi$ (blue), $\Omega=0.5 \pi$ (yellow). Other parameters: $\mu=0.9, q=0.1, \phi_{0}=0$.

interspike-interval density may coexist [Fig. 5.1(a)], while they merge into a very wide bump at higher frequencies.

Signal processing under constrained stimulation may benefit from stochastic resonance as shown in Fig. 5.3. The signal-to-noise ratio (SNR, Eq. 4.70a) is maximized at a particular noise level $\sigma_{\max }$, which increases with stimulus frequency. The mechanism responsible for this effect is best discussed for the low frequency case. When the SNR is plotted versus noise amplitude on a logarithmic abscissa, the graph reveals additional structure, see Fig. 5.4(a). Besides the maximum at $\sigma_{\max }=0.0078$, a marked change in the slope of the curve become visible at $\sigma_{X}=0.027$. This points to a cross-over between two different regimes in the SNR decay. Figure 5.4(b) demonstrates that stochastic resonance is caused by frequency matching in case of constrained stimulation: The signal-to-noise is maximal for that noise amplitude for which the location $\Omega_{\max }$ of the spectral peak (compare Chapter 4.7) coincides with the reset frequency

$$
\Omega_{\mathrm{res}}=2 \pi / T_{\mathrm{res}} .
$$

Here, $T_{\text {res }}$ is the location of the maximum of the ISI density i.e. the single most probable ISI length, as indicated in Fig. 5.5. The stimulus will thus most frequently be reset in intervals of $T_{\text {res }}$, hence the name reset frequency. Coincidence of this frequency with the location of the spectral maximum indicates synchronization between the stimulus reset and the correlations that dominate 

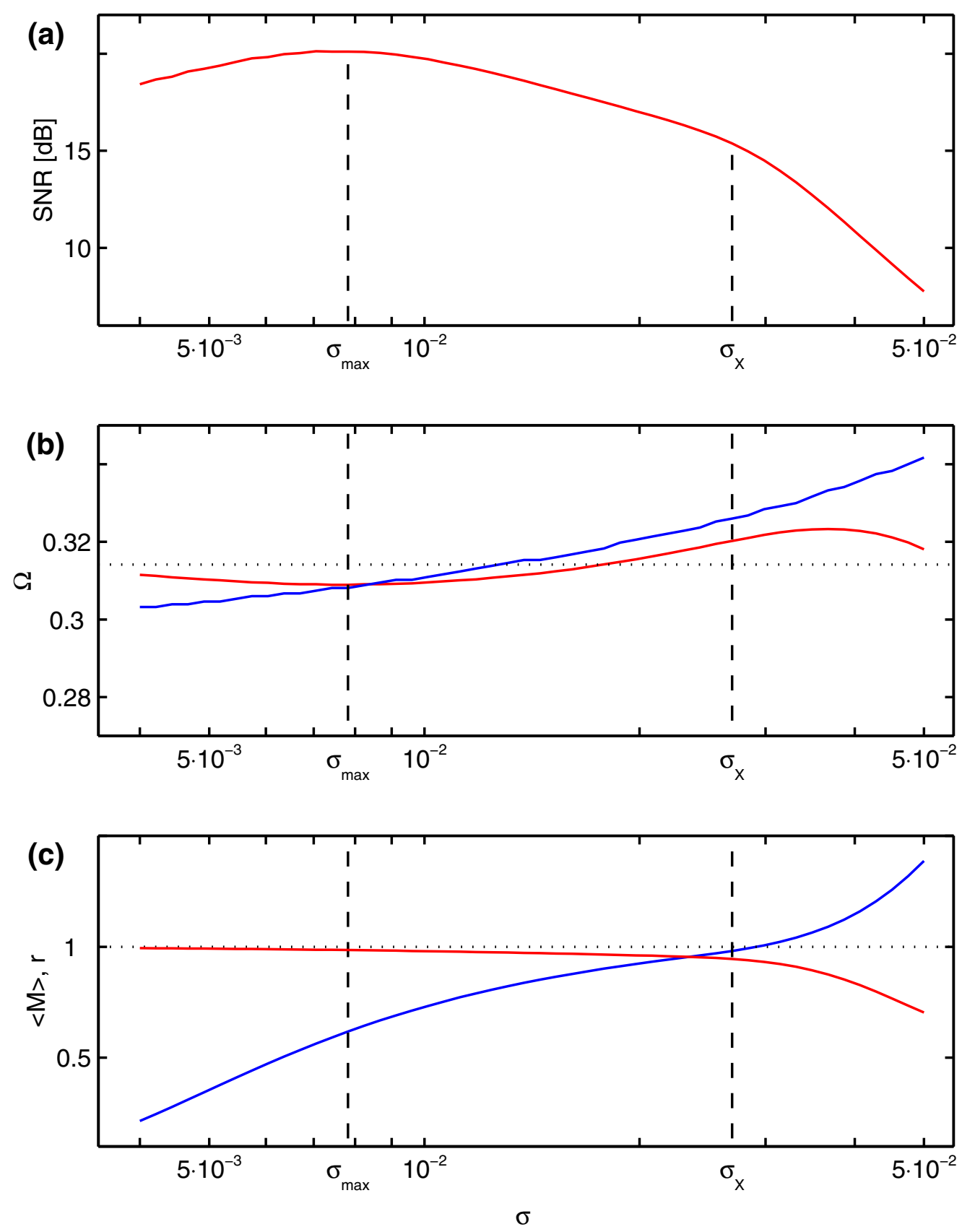

Figure 5.4: The mechanism of stochastic resonance under constrained stimulation: (a) The graph of SNR vs. noise amplitude for low frequency shows more structure when the abscissa is logarithmic (red line in Fig. 5.3). Dashed vertical lines mark the optimal noise $\sigma_{\max }$ and the noise amplitude $\sigma_{X}$ at which cross-over to non-sinusoidal effective stimulation occurs. (b) The SNR is maximized when the location of the spectral peak $\Omega_{\max }$ (red) and reset frequency $\Omega_{\text {res }}$ coincide. The dotted line marks the nominal stimulus frequency $\Omega=0.1 \pi$. (c) If the average number $\langle M\rangle$ of spikes per stimulus period (blue), exceeds unity for $\sigma>\sigma_{X}$, the the effective stimulus is non-sinusoidal, and both vector strength $r$ (red) and SNR are diminished. 


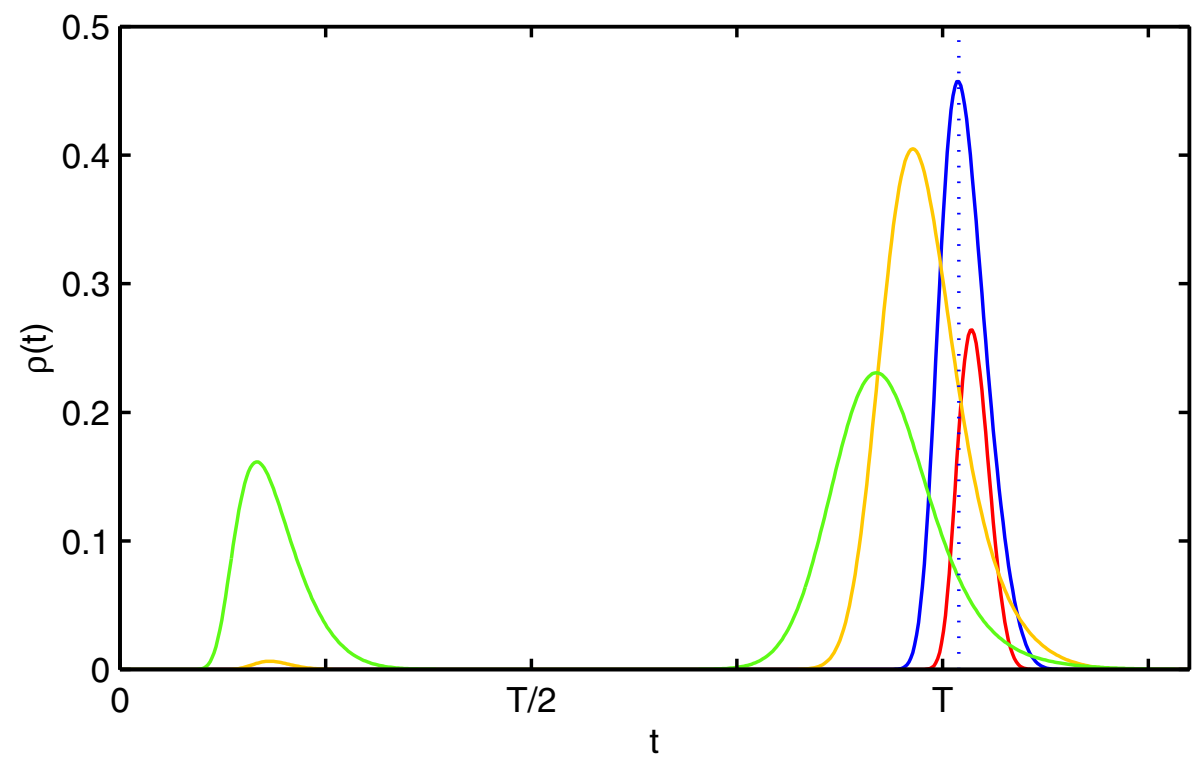

Figure 5.5: ISI densities for the low-frequency stimulus of Fig. 5.3 at different noise amplitudes $\sigma=0.0042$ (red), $\sigma_{\max }=0.0078$ (blue), $\sigma_{X}=0.027$ (yellow), and $\sigma=0.05$ (green). The dotted line marks the reset time $T_{\text {res }}$ for $\sigma=\sigma_{\max }$. The Gaussian peaks of the ISI density near the stimulus period $T$ shift towards shorter times as noise increases. At $\sigma_{X}$, the refractory peak at $t \approx T / 4$ becomes discernible; see text.

the power spectral density. It is plausible that this match should give rise to optimal signal processing performance.

The modes of the ISI density shift towards longer intervals below the optimal noise amplitude $\sigma_{\max }$, and the reset frequency decreases, while it increases beyond $\sigma_{\max }$ as interspike intervals become shorter, see Fig. 5.5. The resulting desynchronization with the spectral peak frequency $\Omega_{\max }$ causes the rather symmetric decay of the SNR below and above the optimal noise amplitude. Beyond the cross-over point $\sigma_{X}$, the SNR drops faster, because for $\sigma>\sigma_{X}$ on average more than one spike is fired per nominal stimulus period as demonstrated in Fig. 5.4(c). This is apparent in the ISI density from the minute refractory mode appearing at $t \approx T / 4$ (yellow line in Fig. 5.5). Since the stimulus is reset after less than a period in this case, the effective stimulus but little resembles the nominal one, and the periodicity of the latter is lost. This is indicated by the marked decay of the vector strength $r$ beyond $\sigma_{X}$ as well.

Stochastic resonance thus exists for a properly chosen initial phase $\phi_{0}=0$. For other values of $\phi_{0}$, with reset times $T_{\text {res }}$ far from the nominal period $T$, the stimulus, and thus the firing pattern of the neuron, may differ markedly from a sinusoid [Fig. 4.2(b)], and the spectrum might not have a peak at all in the vicinity of the nominal stimulus frequency $\Omega$, as exemplified in Fig. 5.2(f). The output of the neuron does not reflect the nominal input in this case.

This raises the issue of how to choose the initial phase $\phi_{0}$ properly. The 

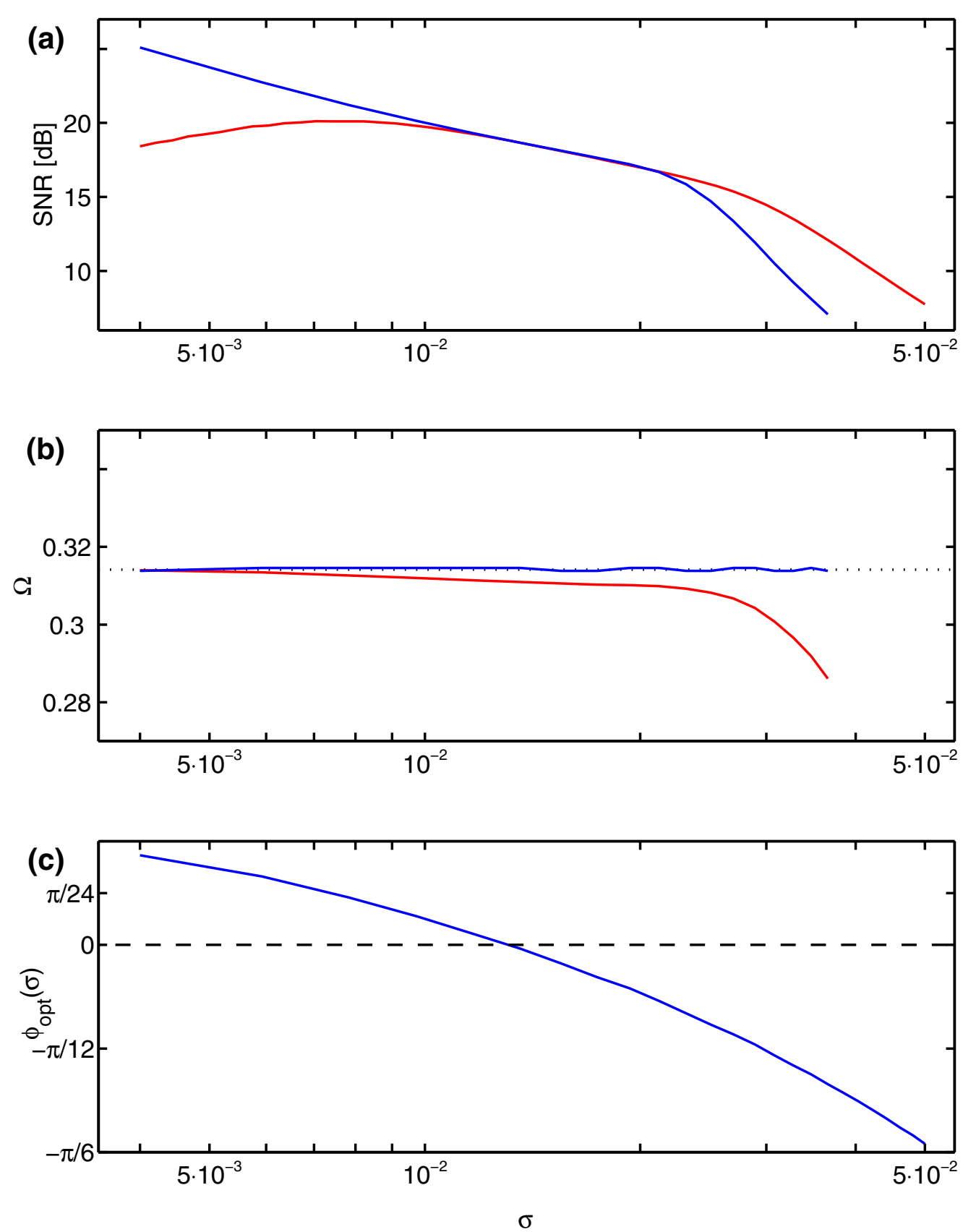

Figure 5.6: Stochastic resonance does not occur if the initial phase $\phi_{0}$ is adapted to the noise level. (a) The signal-to-noise ratio grows linearly as noise decreases if $\phi_{0}=\phi_{\text {opt }}(\sigma)$ (blue). The red curve is the same as in Fig. 5.4(a) for comparison $\left(\phi_{0}=0\right)$. (b) The reset frequency $\Omega_{\text {res }}$ (blue) is virtually identical with the nominal stimulus frequency $\Omega$ if the initial phase is adjusted, while the peak location $\Omega_{\max }$ approaches $\Omega$ from below for $\sigma \rightarrow 0$ (red). (c) Optimal phase $\phi_{\text {opt }}(\sigma)$ vs. noise amplitude; $\phi=0$ is shown dashed for comparison. 
only distinguished phase is that for which spiking will most probably occur at $\phi_{0}$ again, so that the effective stimulus is identical to the nominal on average. This optimal initial phase is given by the location of the mode of the stationary spike phase density

$$
\phi_{\mathrm{opt}}=\underset{\psi \in[0,2 \pi)}{\arg \max } \chi^{(s)}(\psi)
$$

obtained for unconstrained stimulation. This phase ought to be used as initial phase when the response to unconstrained stimulation is approximated as a renewal process. The optimal initial phase depends on the input noise amplitude $\sigma$ as shown in Fig. 5.6(c): For weak noise, the initial stimulus phase is advanced to shorten interspike intervals, while for strong noise the phase is delayed to protract firing. As a consequence, the reset frequency $\Omega_{\text {res }}$ becomes identical to the nominal stimulus frequency $\Omega$ for all noise amplitudes, see Fig. 5.6(b). The signal-to-noise ratio, finally, increases linearly (on the decibel scale) as the input noise vanishes. Thus stochastic resonance does not occur if the proper initial phase $\phi_{\text {opt }}(\sigma)$ is chosen at each noise level.

The conclusion to be drawn from this section is that stochastic resonance occurs in the case of constrained stimulation only if the stimulus phase $\phi_{0}$ is fixed within a narrow range. Outside of this range, the signal is massively distorted, while proper adaptation of the initial phase to the noise present in the input destroys the stochastic resonance effect.

\subsection{Unconstrained stimulation: stationary response}

This section will characterize stationary responses of the Ornstein-Uhlenbeck neuron to unconstrained sinusoidal stimuli to prepare the discussion of stochastic resonance in the following section. The stimuli defined in Table 5.1 will again serve as examples.

Most insight into the firing pattern of the neuron under unconstrained stimulation is provided by the phase transition matrix $\mathbf{T}$, since this matrix contains all information about the synchronization of the spike train to the stimulus phase. The matrices for the sample stimuli are displayed in Fig. 5.8. The only important information lacking from the transition matrix is whether subsequent spikes occur within the same stimulus period or not. When noise is weak (left column of Fig. 5.8), the transition matrices are characterized by a horizontal bar of high transition probability, which intersects the matrix diagonal. Thus, a stochastic fixed point exists. Spikes will preferentially occur at this fixed point with not more than one spike per stimulus period. The resulting stationary spike phase density $\chi^{(s)}$ is sharply peaked (red lines in center colum of Fig. 5.9). The pertaining ISI densities confirm this analysis: spikes are 
(a)

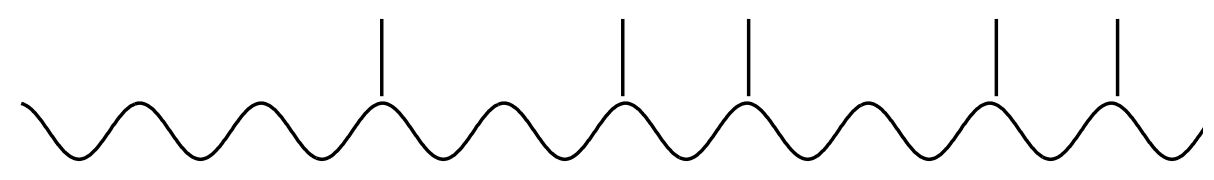

(b)

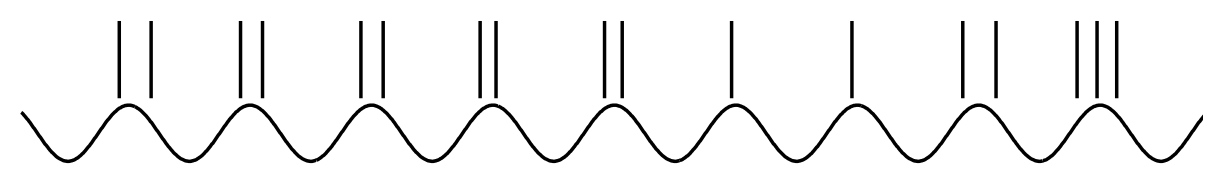

(c)

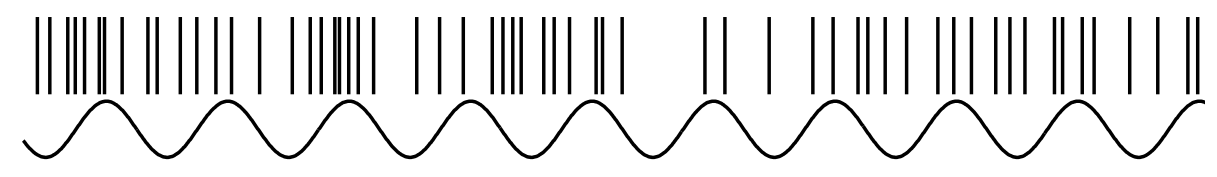

Figure 5.7: Characteristic firing patterns in response to unconstrained stimulation: (a) Phase locking with occasional skipping of a period, (b) bursts of two to three spikes interrupted by silence, and (c) dithered firing. The stimulus is the low frequency stimulus of Table 5.1 at weak, medium and strong noise.

separated by multiples of the stimulus period. They also indicate that several periods may be skipped in between spikes. Note that the modes of the stationary ISI density are of necessity centered about multiples of the stimulus period $T$ : otherwise, no sharp spike phase density could result. The corresponding spike intensities $q(t)$ are shown on the right-hand side of Fig. 5.9. They clearly demonstrate the precise locking of the spikes to a particular stimulus phase. That the neuron may skip stimulus periods is indicated by the amplitude of the spike intensity: the integral of $q(t)$ over a single peak (say the one around $t=T)$ gives the average number of spikes fired during the peak $(\approx 0.73$ in this case). The spike intensity takes on a stationary, periodic pattern rapidly, usually within a single stimulus period. All discussions of the spike intensity will therefore refer to this stationary pattern. A sample spike train for low noise is shown in Fig. 5.7(a). Phase-locked responses of this type may serve as input to coincidence detectors measuring phase differences between stimuli arriving from both ears, and thus form the basis of stereophonic hearing (Gerstner et al. 1996).

Bursting firing patterns occur at low frequencies combined with intermediate noise amplitudes. In this case, the transition matrix has a structure as shown in Fig. 5.8(b). The horizontal high-probability bar no longer intersects the matrix diagonal, but extends a "finger" parallel to it within a part of the phase axis. As discussed in Chapter 4.4.2, this indicates that a few spikes may be fired in rapid succession, interrupted by a pause when the firing phase returns from the tip of the finger to the horizontal bar. For the given parameters, the burst will typically consist of just two spikes, see Fig. 5.7(b). The corresponding stationary spike phase density [blue in Fig. 5.9(b)] has two maxima in consequence, while the pertaining ISI density is characterized by a narrow 


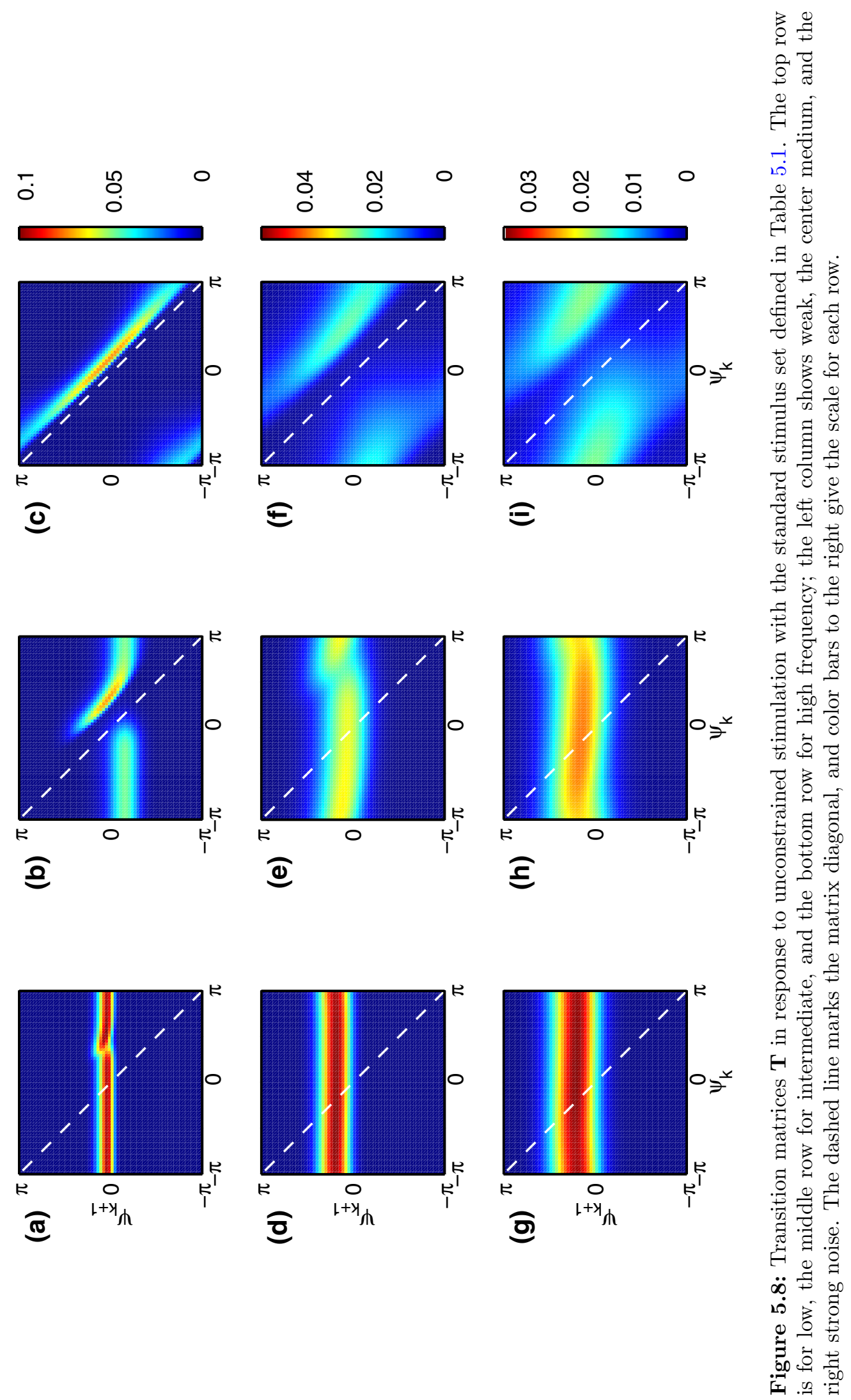




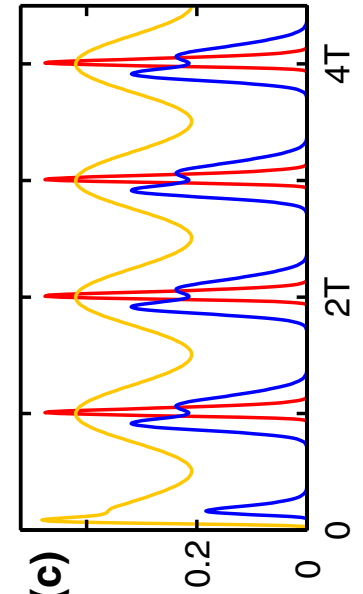

(l) $b$

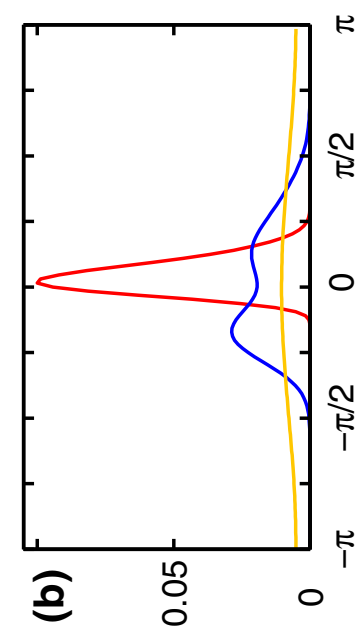

(h) $)_{(s)} \chi$

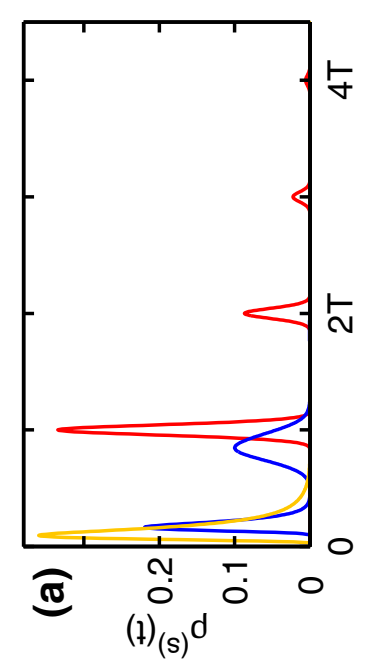

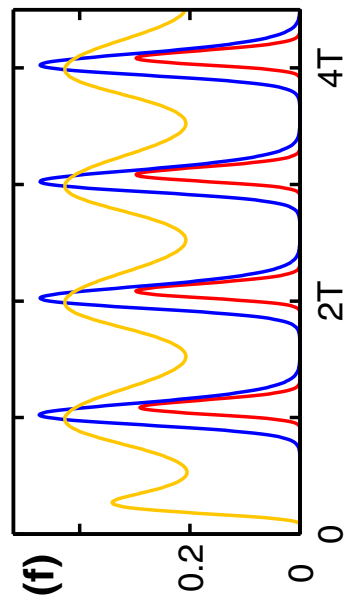

(l) $\mathrm{b}$

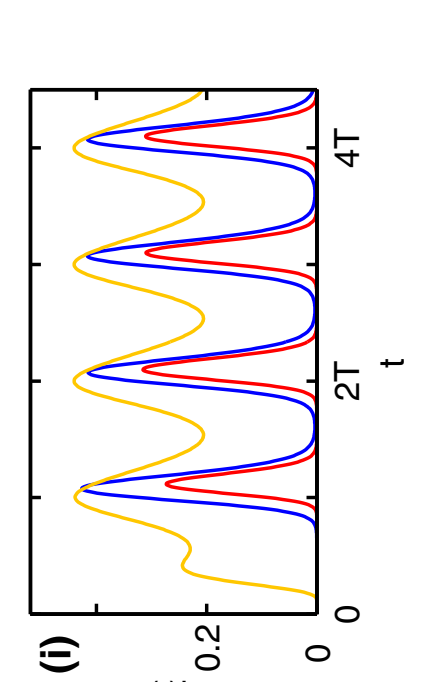

(l) $\mathrm{b}$

黄总

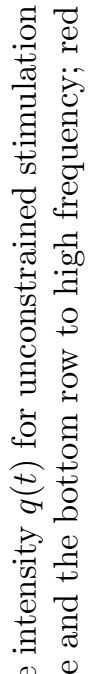

营

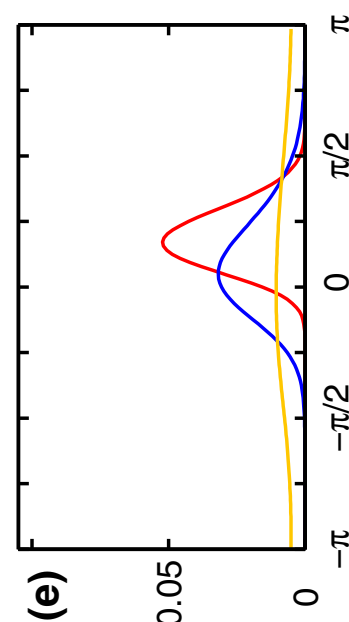

$(\pitchfork)_{(\mathrm{s})} \chi$

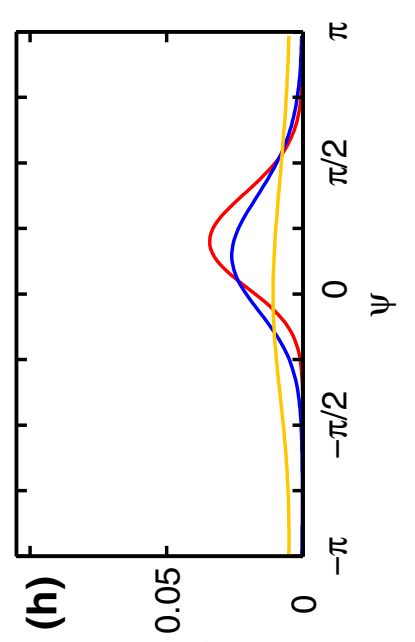

(h) ${ }_{(\mathrm{s})} \chi$
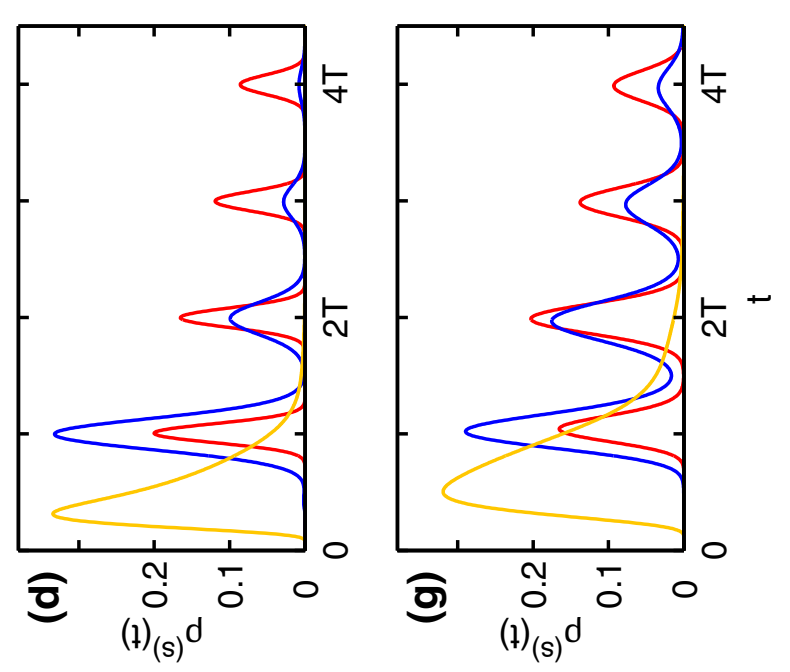

శี

(ิ).

불

()

永章

$\times \cdot \bar{a}$

密

包

)

क्षे

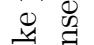

ही

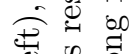

过

ब者

के

B.

政

可

氙要

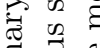

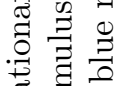

要

$\ddot{0}:$

is

讯

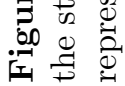



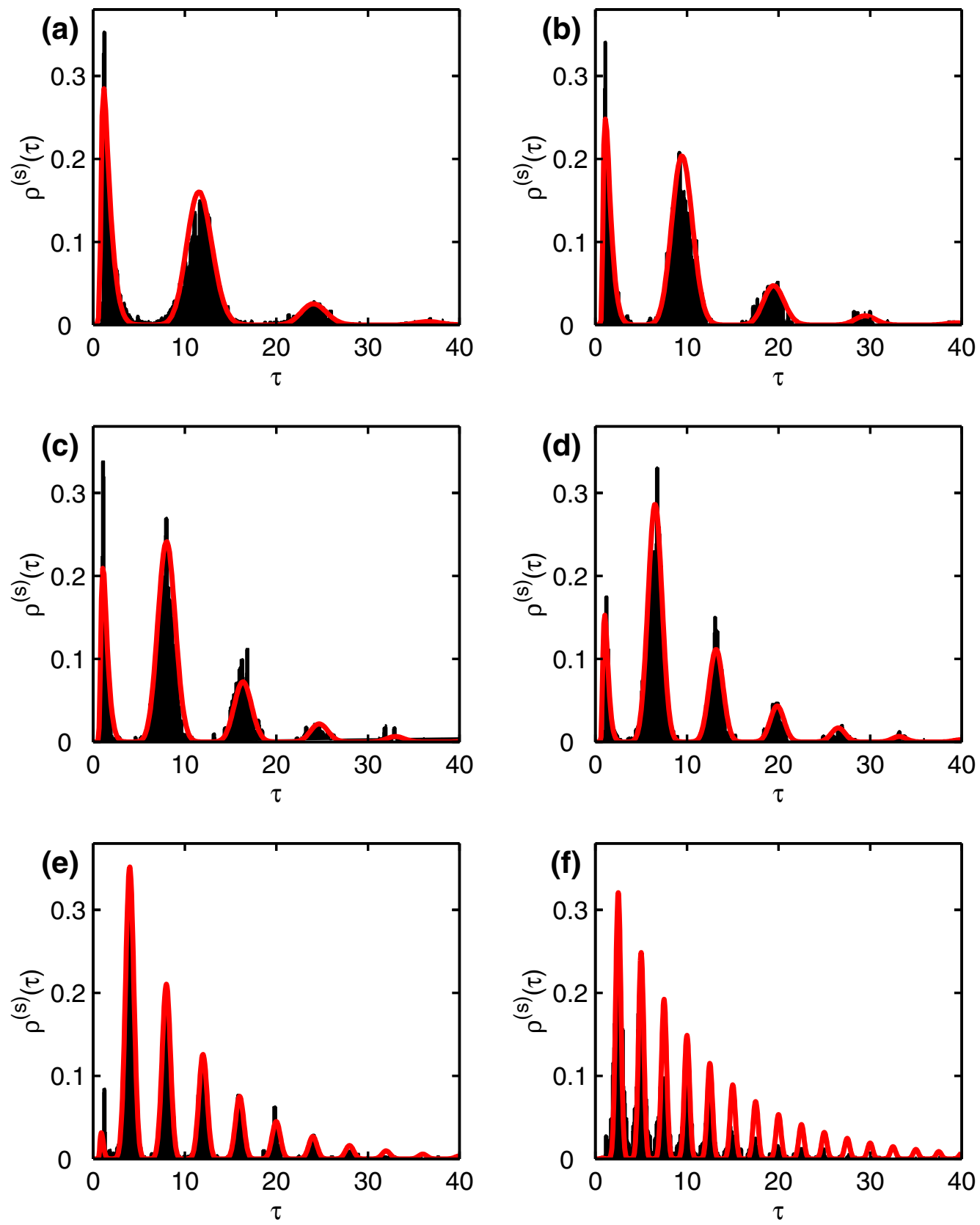

Figure 5.10: ISI histograms recorded from a monkey auditory nerve neuron a with best frequency of $400 \mathrm{~Hz}$ (black) and stationary ISI densities $\rho^{(s)}(t)$ from the Ornstein-Uhlenbeck neuron in response to pure sinusoids with frequencies (a) $80 \mathrm{~Hz}$, (b) $100 \mathrm{~Hz}$, (c) $120 \mathrm{~Hz}$, (d) $150 \mathrm{~Hz}$, (e) $250 \mathrm{~Hz}$, and (f) $400 \mathrm{~Hz}$, which were scaled to dimensionless units with a membrane time constant of $\tau_{m}=237 \mu \mathrm{s}$, cf. Eq. (2.19). Model parameters were $\mu=0.86$, $q=0.08, \sigma=0.051$, and $v_{R}=0$ for all frequencies. Experimental data was digitized from Fig. 4 of Rose et al. (1967). 
intra-burst peak at short times and a wider inter-burst peak close to the stimulus period $T$. The spike intensity reflects the bursting firing pattern with two preferred firing times per period. The integral over a single peak is $\approx 1.75$ here, i.e. on average a two-spike burst is fired in three out of four periods, and only a single spike in the other cases. Bursts may comprise a large number of spikes if the stimulus frequency is sufficiently low, see Fig. 5.12(1). In this case, the spike intensity does not necessarily show a maximum for each spike within a burst but may be smeared into a peak without substructure. Bursting cannot occur for intermediate to high frequencies with periods close to or shorter than the refractory time of the neuron. In this case, the stochastic fix point simply blurs with increasing noise and firing is gradually less phase-locked as the strong noise regime is approached, cf. Fig. 5.8(e) and (h).

For strong noise, the transition matrix assumes a rather different structure independent of the stimulus frequency, as shown in the right column of Fig. 5.8. It is dominated by a band running parallel to and above the matrix diagonal, with a companion in the lower left corner. If the matrix were wrapped to form a torus, these two structures would join to form a band wound around the torus. Figuratively speaking, spikes will "run" along this band. Firing is thus likely at any phase, although the stationary phase density retains a weak maximum (yellow in Fig. 5.9, center column). The ISI density shows little sign of of the underlying periodic stimulus, whereas the spike intensity is a near-perfect copy of the sinusoidal input to the neuron: the neuron's response is linearized by strong noise. Linearization by noise is known in electrical engineering as dithering, and employed to alleviate discretization errors in digital-to-analog converters (Gammaitoni 1995). Therefore, this regime will be referred to as dithered firing. A sample spike train is shown in Fig. 5.7(c). In the cortex, pools of neurons operating in this regime may provide the "carrier waves" called for by some models of neural coding (Hopfield 1995).

A test of the Ornstein-Uhlenbeck neuron against neurophysiological data is given in Fig. 5.10. The histograms drawn in black are interspike-interval distributions recorded from the auditory nerve fiber of squirrel monkeys (Rose et al. 1967). The stimuli were pure sinusoidal tones delivered to the ear of the animal. The ISI distribution consists only of peaks at multiples of the stimulus for high stimulus frequencies [Fig. 5.10(f)], while for lower frequencies a refractory (intra-burst) peak appears at short times and becomes dominant at the lowest frequencies. The Ornstein-Uhlenbeck neuron reproduces this behavior faithfully with just a single set of parameters. They were optimized globally over all six frequencies (The MathWorks, Inc. 1999). The OrnsteinUhlenbeck neuron thus appears to capture the essential properties of the spike generator in neurons of the auditory nerve tuned to low frequencies. Two details should be pointed out: First, the membrane time constant $\tau_{m} \approx 237 \mu \mathrm{s}$ may seem to short to be biologically plausible, but effective time constants in this range have been found in auditory neurons of birds (Gerstner et al. 1996; 

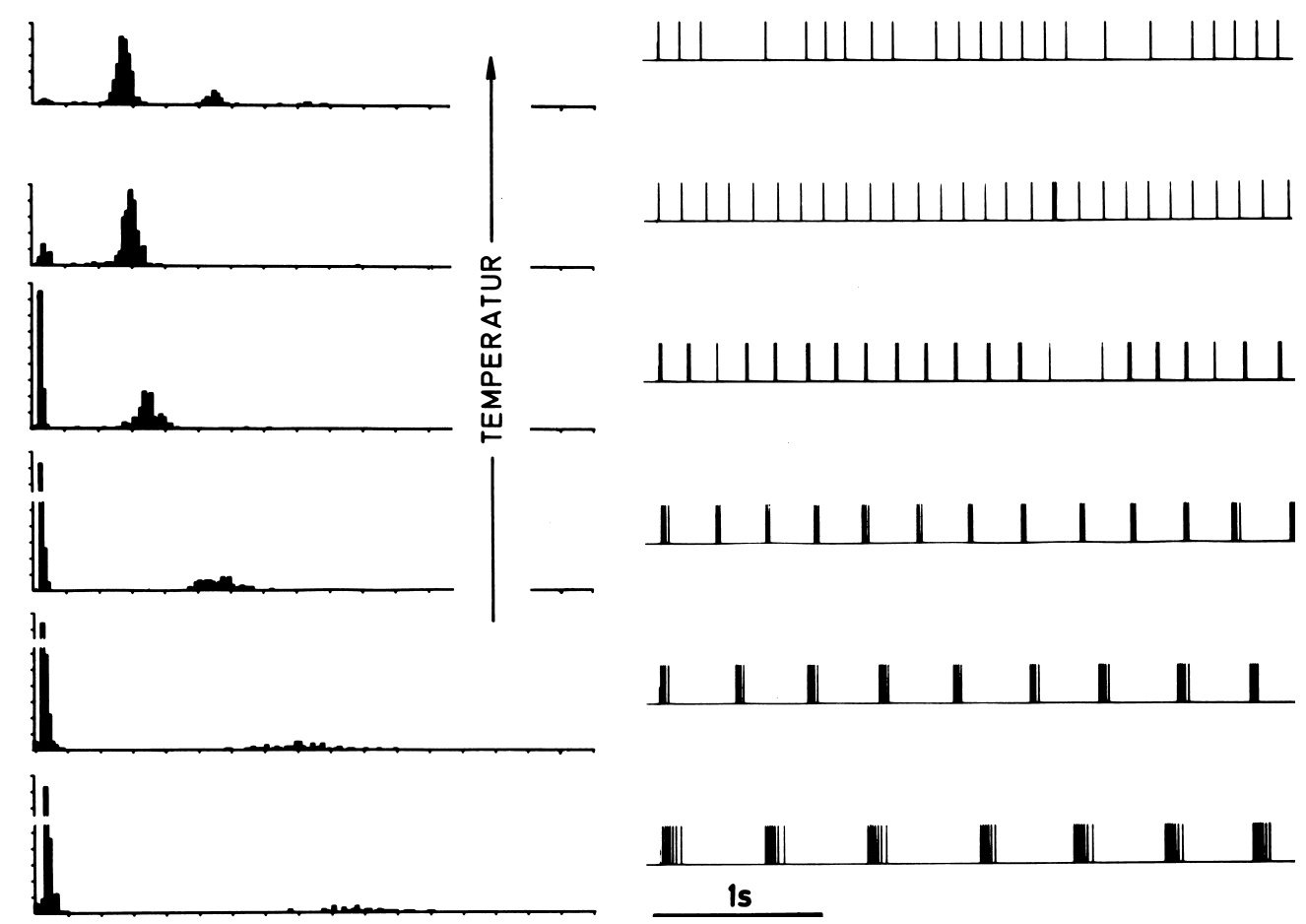

Figure 5.11: Stationary discharge patterns of bursting cold receptors of the cat lingual nerve at different constant temperatures. ISI histograms are shown on the left, and schematic spike trains are to the right; the tick marks on the ISI histogram abscissae are $50 \mathrm{~ms}$ apart [adapted from Fig. 5 of Braun et al. (1984); with permission of the authors and Wiley-VCH].

Geisler 1968). Second, the fit shown was obtained for $v_{R}=0$ indicating that this is a reasonable value for the reset potential in sensory afferent neurons. Cortical neurons, on the other hand, appear to experience much smaller resets after spikes (Troyer and Miller 1997); the "cortical" parameter regime will briefly be discussed in Section 5.3.3.

Further evidence in favor of the Ornstein-Uhlenbeck neuron is provided by a comparison of Figures 5.11 and 5.12. The ISI histograms and spike trains shown in the former were recorded from the lingual nerve of cats, a cold receptor afferent neuron. This nerve discharges spikes in bursts separated by long gaps at low temperature $(\mathrm{T} \approx 15 \mathrm{C}$ ). As temperature rises, the burst become shorter but closer, until only single spikes are fired at regular intervals. At even higher temperatures $(\mathrm{T} \approx 35 \mathrm{C})$, an increasing number of spikes are skipped. The Ornstein-Uhlenbeck neuron models this behavior well. The membrane time constant is close to $5 \mathrm{~ms}$ in this case, which is plausible for non-auditory neurons. The cross-over from bursts via regular to skipping firing patterns has been traced to subthreshold membrane potential oscillations generated autonomously in the lingual nerve (Longtin and Hinzer 1996). These endogenous oscillations give rise to a firing pattern that is obviously not a renewal process, whence the term constrained stimulation was introduced in Chapter 4 as a replacement for Lansky's terminology. 
(a)

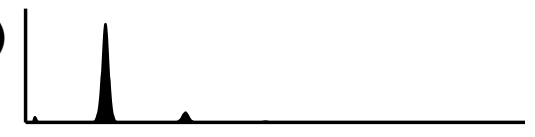

(c)

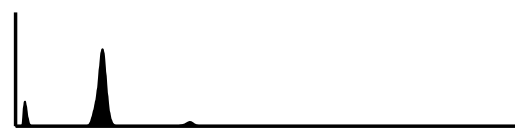

(e)

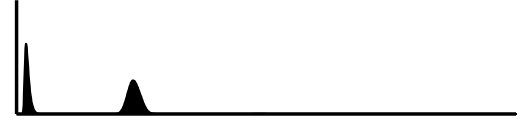

(g)

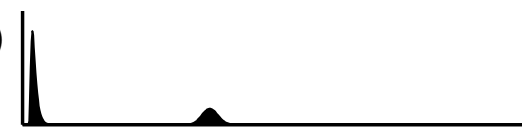

(i)

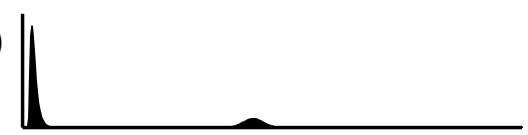

(k)

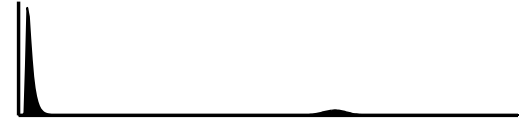

(b)

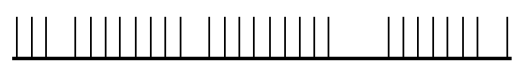

(d)

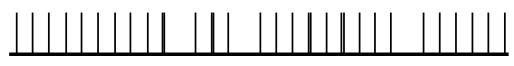

(f)

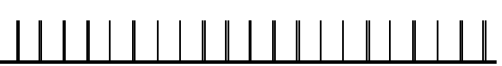

(h)

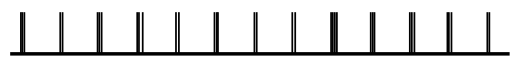

(j)

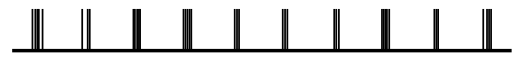

(l)

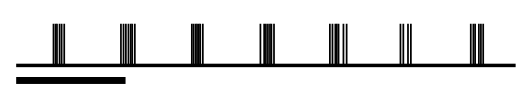

Figure 5.12: ISI densities (left) and firing patterns (right) of the Ornstein-Uhlenbeck neuron reproduce the behavior of cat cold receptors shown in Fig. 5.11 well. ISI density graphs and the scale bar have the same respective widths as those in Fig. 5.11. Stimulus parameters were determined by visual optimization: $\mu=0.6, q=0.4, v_{R}=0, \Omega / \pi=$ $0.071,0.065,0.045,0.027,0.021,0.015$, and $\sigma=0.036,0.042,0.048,0.048,0.06,0.06$ from top to bottom; time is in units of $\tau_{m} \approx 4.8 \mathrm{~ms}$.

The excellent agreement between the Ornstein-Uhlenbeck neuron and experimental data from two species indicates that it is justified to ignore the bounds on hyperpolarization, i.e. to set $v_{\text {hyp }}=-\infty$. This arises because the base current $\mu$ is sufficiently strong in both cases to raise the membrane potential to a level far above any rectifying reversal potentials.

To summarize, the Ornstein-Uhlenbeck neuron is backed by experimental evidence, making it an object worthwhile of investigation.

\subsection{Stochastic double resonance}

The encoding of unconstrained periodic stimuli is now evaluated by measuring the power spectral density and the resulting signal-to-noise ratio as defined in Chapter 4.7. The spikes in a train show a high degree of correlation among each other if they are strongly correlated to the stimulus. As a consequence, the power spectral density has prominent peaks as displayed in Fig. 5.13(a) for the weak noise sample stimulus at $\Omega=0.33 \pi$. The small phase jitter in the phase-locked firing pattern observed in this case preserves correlations 

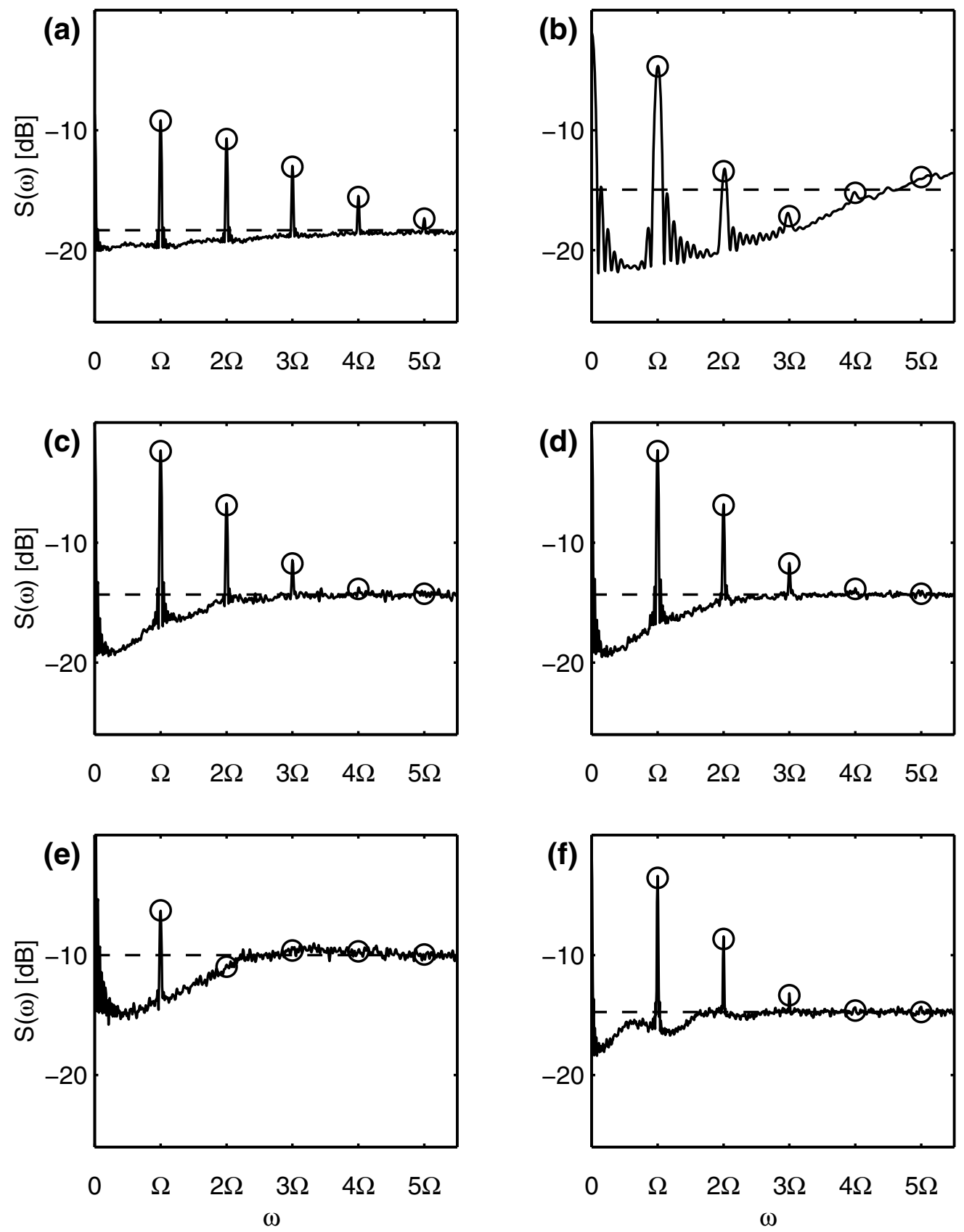

Figure 5.13: The power spectral density in response to unconstrained periodic stimulation exhibits stochastic resonance. Spectra in the left column are for stimulus frequency $\Omega=$ $0.33 \pi$ and noise amplitudes $\sigma=0.03,0.064$, and 0.25 from top to bottom. The observation time is $T_{o}=200 . \mathrm{SNR}_{T_{o}}$ is defined as the height of the spectral peak at $\Omega$ over the dashed Poisson background $S_{P}$. Circles mark results from the Markov chain method, full spectra are from simulations. The right column demonstrates bona fide resonance with maximum $\mathrm{SNR}_{T_{o}}$ for $\Omega=0.33 \pi$ in (d); frequency is $0.1 \pi$ in (b), $0.33 \pi$ in (d), and $0.5 \pi$ in (f), while $\sigma=0.064$ for all frequencies. Remaining parameters are $\mu=0.9, q=0.1, v_{R}=0$. 

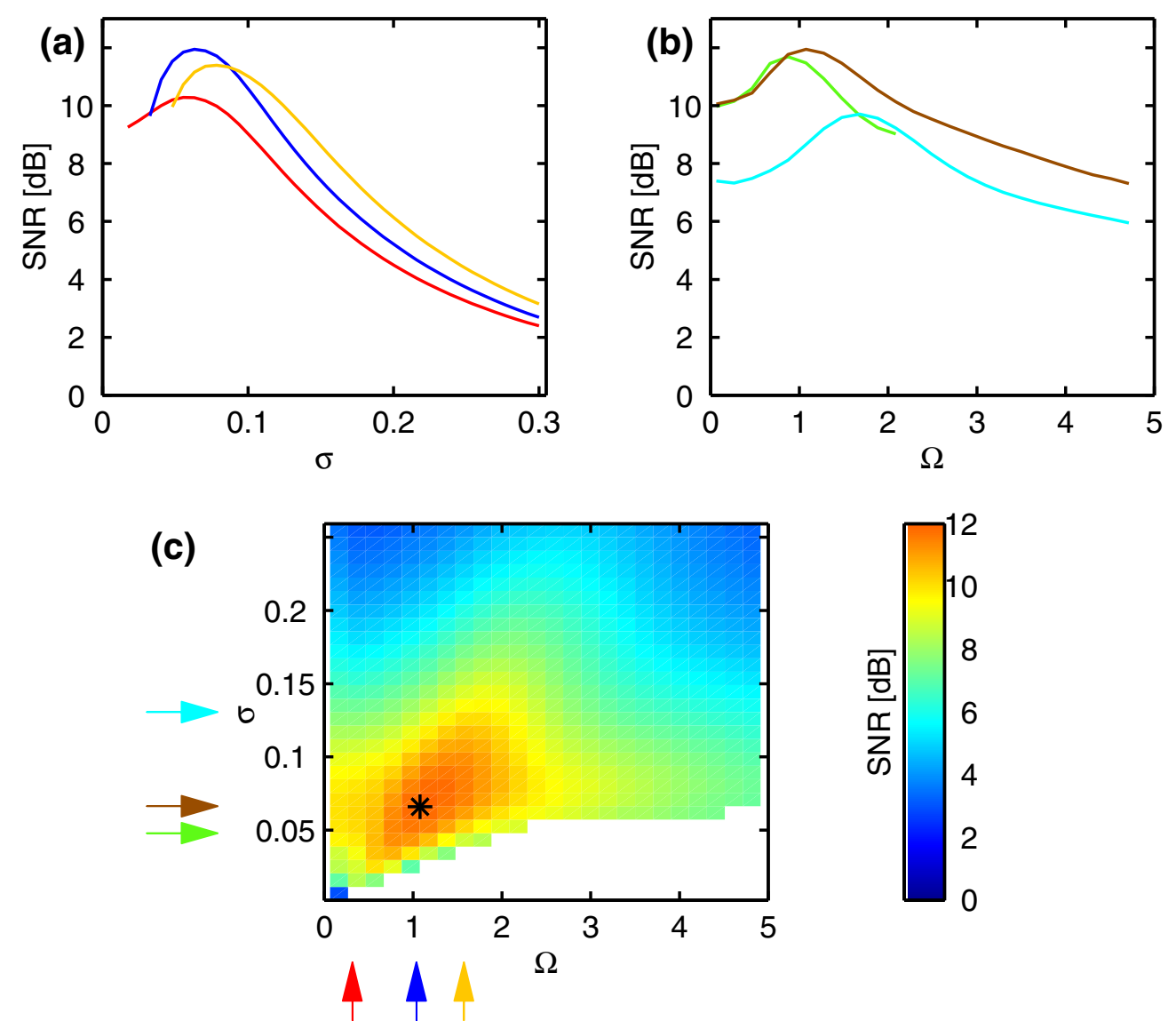

Figure 5.14: In response to unconstrained sinusoidal stimulation, the signal-to-noise ratio shows stochastic double resonance in input noise amplitude $\sigma$ and stimulus frequency $\Omega$. (a) SNR vs. noise for stimulus frequencies $\Omega=0.1 \pi$ (red), $0.33 \pi$ (blue) and $0.5 \pi$ (yellow). (b) SNR vs. stimulus frequency for noise amplitudes $\sigma=0.048$ (green), 0.066 (brown), and 0.131 (cyan). (c) Signal-to-noise ratio as function of both stimulus frequency (abscissa) and noise amplitude (ordinate); SNR is color coded as indicated. The black asterisk marks the maximal $\mathrm{SNR}^{\mathrm{opt}}=12 \mathrm{~dB}$, and colored arrows indicate the parameters used in (a) and (b). Remaining stimulus parameters were $\mu=0.9, q=0.1, v_{R}=0$.

well across many spikes, whence the spectral peaks decay only slowly towards higher harmonics. These long-range correlations are gradually destroyed as noise increases, until just a single peak at the stimulus frequency remains. A glance down the left column of Fig. 5.13 indicates stochastic resonance: the height of the spectral peak at the stimulus frequency above the Poisson background (dashed line) is maximal in Fig. 5.13(c), i.e. for an intermediate noise amplitude $\sigma_{\max }$. An inspection of the right column reveals another kind of stochastic resonance: for fixed input noise amplitude $\sigma$, the signal-to-noise ratio (a difference on the decibel scale) attains a maximum at a preferred frequency $\Omega_{\max }$ (Fig. 5.13(d)). Gammaitoni et al. (1995) has coined the term bona fide stochastic resonance for this effect. 
This observation is manifested in Fig. 5.14. Classic stochastic resonance is demonstrated in Fig. 5.14(a) for the three stimulus frequencies of the sample set: the signal-to-noise ratio shows a clear maximum as a function of the input noise amplitude $\sigma$. The optimal noise levels $\sigma_{\max }$ are comparable to the stimulus amplitude $q=0.1$. Bona fide stochastic resonance is demonstrated in Fig. 5.14(b) which displays maxima of the SNR as a function of the stimulus frequency $\Omega$ for three noise amplitudes. Resonance with respect to both noise amplitude and stimulus frequency is summarized in Fig. 5.14(c). The signalto-noise ratio pertaining to each noise-frequency combination is indicated in color, ranging from blue to deep orange. The signal-to-noise ratio is markedly increased in a small region of the $\sigma-\Omega$ plane and reaches its absolute maximum of $\mathrm{SNR}^{\mathrm{opt}}=12 \mathrm{~dB}$ for $\sigma^{\mathrm{opt}}=0.066$ and $\Omega^{\mathrm{opt}}=1.08=0.342 \pi$ (asterisk). The maximum was found with the aid of a two-dimensional Nelder-Mead minimization algorithm (The MathWorks, Inc. 1999).

This is the central result of this thesis: The encoding of periodic signals into spike trains by the Ornstein-Uhlenbeck neuron is fostered by noise through stochastic double resonance.

\subsubsection{Mechanism}

The expression for the power spectral density at the stimulus harmonics as derived by the Markov chain approach in Chapter 4.6.2 provides a transparent approximation to the signal-to-noise ratio. Inserting Eq. (4.48) into the definition of the SNR (Eq. 4.70b) one obtains

$$
\mathrm{SNR}_{T_{o}}=1+A\left(1, \frac{T_{o}}{\langle\tau\rangle}\right)+\left(\frac{T_{o}}{\langle\tau\rangle}-1\right) B(1) .
$$

As long as all eigenvalues of the transition matrix $\mathbf{T}$ are small (except the largest, which is $\lambda_{1}=1$ by definition), the continuous contribution $A\left(1, T_{o} /\langle\tau\rangle\right)$ will be negligible, while the singular part (Eq. 4.61)

$$
B(1)=\left|\sum_{j=0}^{L-1} \chi_{j}^{(s)} \mathrm{e}^{i j \Delta \psi}\right|^{2}=r^{2}
$$

is the square of the vector strength. The latter is close to unity for noise not too strong, so that ${ }^{2}$

$$
\mathrm{SNR}_{T_{o}} \approx \frac{T_{o}}{\langle\tau\rangle} r^{2}=\nu r^{2} T_{o}
$$

\footnotetext{
${ }^{2}$ This model amounts to treating the spike train as an inhomogeneous Poisson process (Shimokawa et al. 1999b). The phenomenological Ansatz $R_{\text {phen }}$ proposed by Plesser and Geisel (1999) is the square root of the approximation given here.
} 

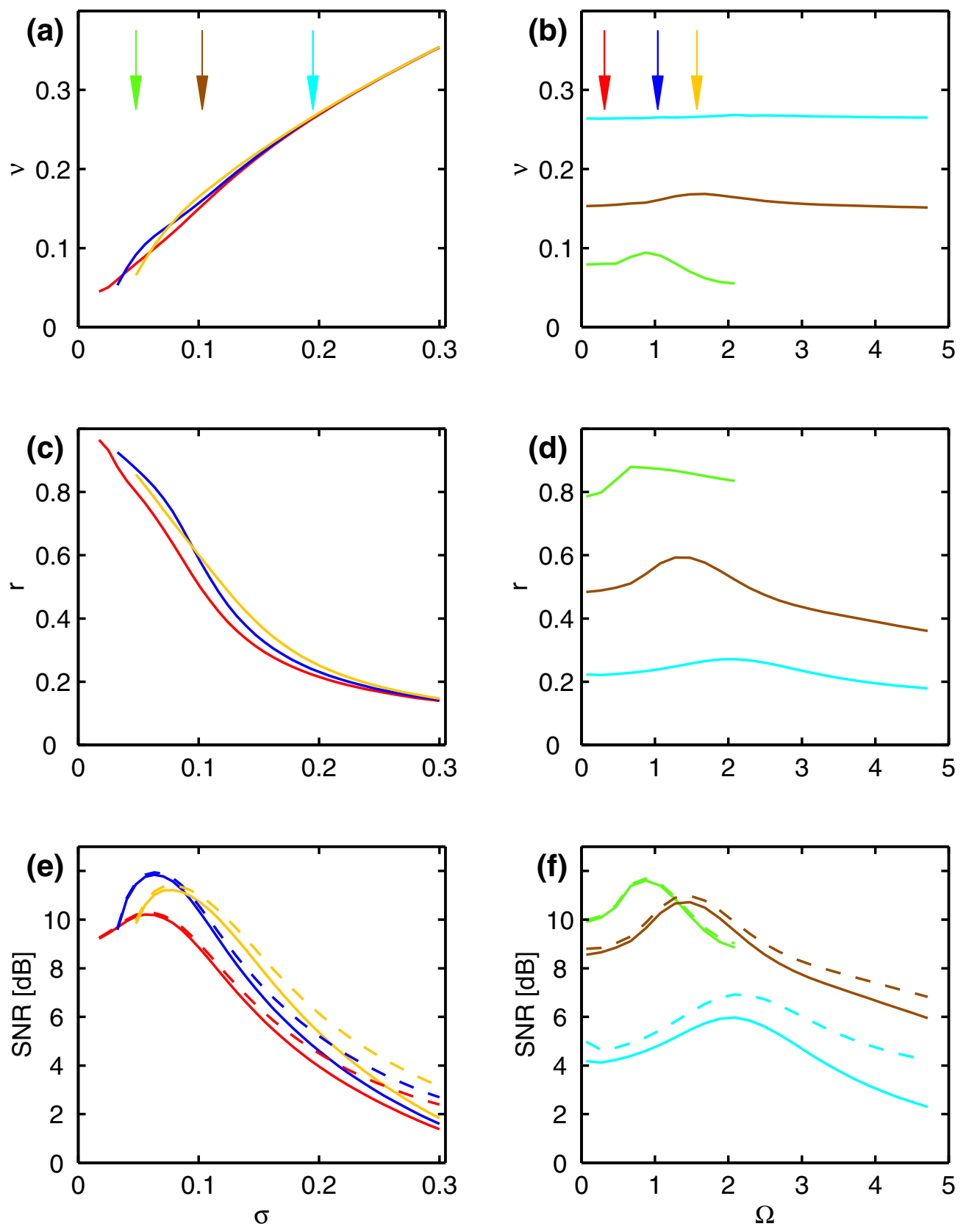

Figure 5.15: Stochastic resonance results from a compromise between signal intensity [firing rate $\nu,(\mathrm{a})$ and (b)] and signal quality [vector strength $r,(\mathrm{c})$ and (d)]: the signal-to-noise ratio is approximately $\mathrm{SNR} \approx \nu r^{2} T_{o}$ [full lines in $(\mathrm{e})$ and (f)]. Exact results from the Markov chain are shown as dashed lines. Data are plotted vs. noise amplitude $\sigma$ on the left for frequencies $\Omega=0.1 \pi$ (red), $0.33 \pi$ (blue), and $0.5 \pi$ (yellow), and vs. stimulus frequency $\Omega$ on the right for noise amplitudes $\sigma=0.048$ (green), 0.103 (brown) and 0.195 (cyan). Colored arrows on the left mark the noise amplitudes used on the right and vice versa. 

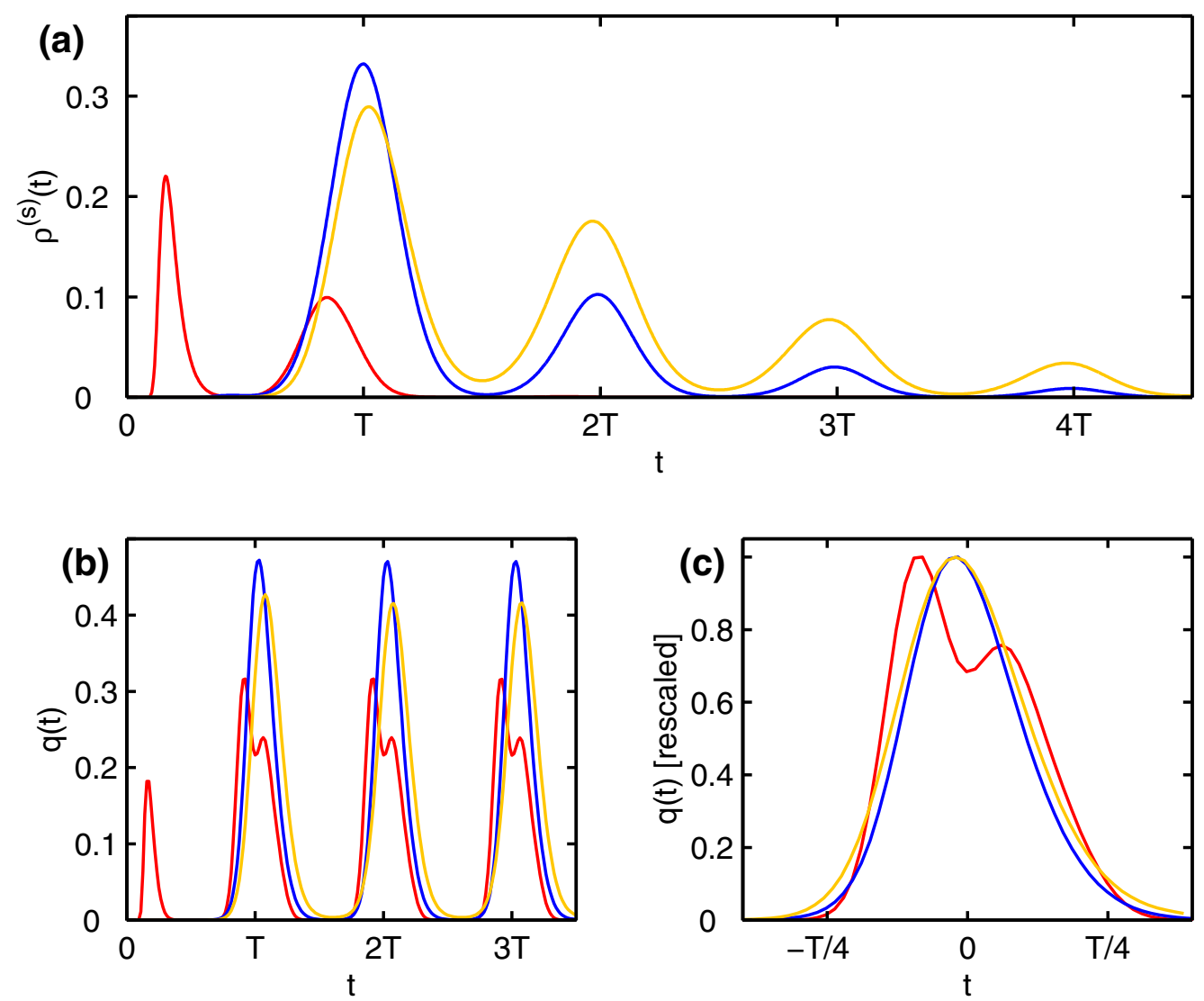

Figure 5.16: Matching of timescales gives rise to the bona fide resonance with respect to the stimulus frequency. (a) Stationary ISI density $\rho^{(s)}(t)$ at the optimal noise amplitude $\sigma_{\max }$ for low $\left(\Omega=0.1 \pi\right.$, red), optimal ( $\Omega^{\mathrm{opt}}=0.342 \pi$, blue) and high frequency $(\Omega=0.5 \pi$, yellow). The pertaining spike intensities $q(t)$ are given in (b). (c) The peaks of the spike intensity at the first stimulus period shifted and rescaled for comparison of their widths at half height. Amplitudes in (a) and (b) are misleading since abscissae are given in multiples of the stimulus frequencies to facilitate comparison $(T=20,5.8,4$, resp.). The integral of the peaks near $T$ in (b) is $1.74,0.69$, and 0.48 for the red, blue and yellow curves, respectively.

This approximation holds well as shown in Figs. 5.15(e) and (f). The signal-tonoise ratio is thus expressed in terms of two quantities permitting an intuitive access, namely the firing rate $\nu$, quantifying signal intensity and the vector strength $r$, measuring signal quality. The left column of Fig. 5.15 demonstrates that the classic stochastic resonance with respect to noise amplitude results from a compromise between these two signal properties: the optimal combination of signal intensity and signal quality yields stochastic resonance.

The resonance in stimulus frequency, in contrast, is mainly caused by a maximum of the vector strength as a function of stimulus frequency, see Fig. 5.15(d). This holds particularly for stronger noise, when the firing rate is virtually independent of the stimulus frequency [cyan line in Fig. 5.15(b)]. For 
the optimal stimulus-noise combination, both $\nu$ and $r$ are maximized. This synchronization resonance of the vector strength as a function of stimulus amplitude arises by matching the stimulus period to the internal timescale of the neuron, which is set by its refractory time $\tau_{\text {ref }}$ as shown in Fig. 5.16(a). For low frequency ( $\Omega=0.1 \pi$, red), the neuron fires in bursts [cf. Fig. 5.8(a) for the transition matrix], with a stationary interspike-interval density $\rho^{(s)}(t)$ characterized by the intra-burst peak near $t \approx \tau_{\text {ref }}$ and the inter-burst peak close to the stimulus period. This gives rise to rather wide peaks of the spike intensity as shown in Fig. 5.16(c). If the stimulus is faster than optimal (yellow), phase locking is lost and firing possible at all times. The modes of the ISI density are slightly delayed compared to the stimulus period $T$, indicating that the refractory time is longer than the period. The peaks of the spike intensity are rather broad in this case as well. At the optimal frequency (blue), the modes of the ISI density are perfectly aligned with the stimulus period and well separated. This results in narrow peaks of the spike intensity separated by intervals of silence: phase-locking is optimal if the timescales of stimulus and neuron are matched.

The analysis above indicates that the signal-to-noise ratio as a measure of coding quality "rewards" phase-locked responses with a large vector strength, i.e. rythmic firing patterns. In contrast, quantities measuring the correlation between stimulus and response will reward dithered firing with nearly sinusoidal spike intensity (Collins et al. 1996; Chialvo et al. 1997). From a neuroscience perspective, the signal-to-noise ratio appears preferable for two reasons: First, any processing based on coincidence detection, such as in stereophonic hearing, requires phase-locked spike trains (Gerstner et al. 1996). Second, it appears that spike packets can only propagate through neural networks if they are both sufficiently strong and "tight" (Diesmann et al. 1999).

\subsubsection{Role of stimulus amplitude and base current}

All results up to this point were obtained for a single combination of base current and stimulus amplitude. Figure 5.17 extends this to 24 combinations of base currents $(0.6 \leq \mu \leq 0.95)$ and stimulus amplitudes $(0.4 \leq q /(1-\mu) \leq$ 1.2). The reset potential was kept fixed at $v_{R}=0$, as appropriate for afferent neurons, cf. Section 5.2. For each case, the signal-to-noise ratio is displayed in color as a function of stimulus frequency $\Omega$ and input noise amplitude $\sigma /(1-\mu)$ as in Fig. 5.14(c). Stochastic double resonance is found for all parameter combinations, although less pronounced for small base currents (top row). The optimal frequency-noise combination is again marked by an asterisk. Stochastic double resonance is thus firmly established for the Ornstein-Uhlenbeck neuron in the "afferent" regime.

The dependence of the optimal signal-to-noise ratio $\mathrm{SNR}^{\mathrm{opt}}$, frequency $\Omega^{\mathrm{opt}}$ and noise amplitude $\sigma^{\text {opt }}$ on base current $\mu$ and stimulus amplitude $q$ is best 

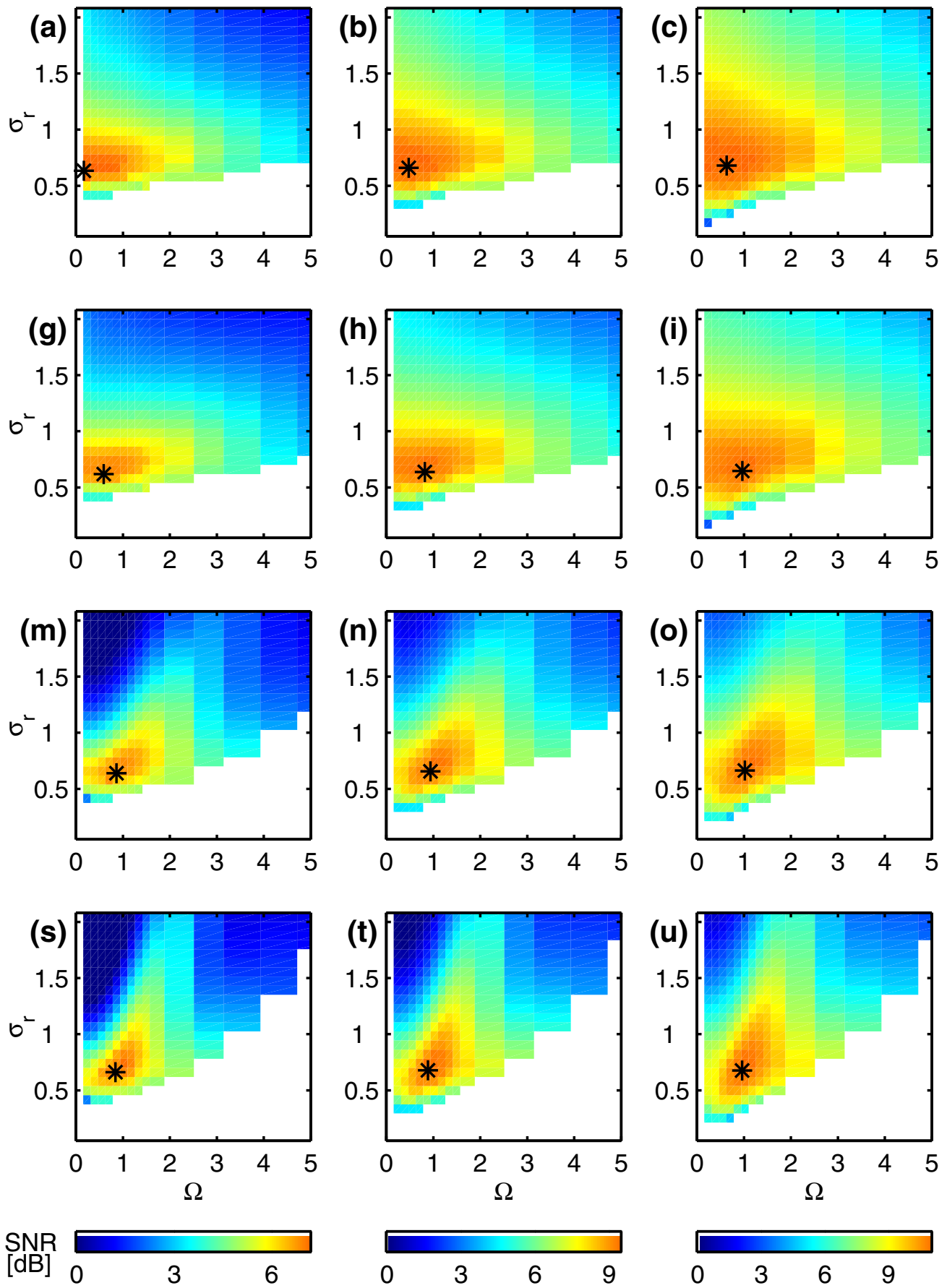

Figure 5.17: Signal-to-noise ratio for various combinations of base current current and relative stimulus amplitude. Each figure shows the signal-to-noise ratio in color for one $\mu$ - $q$-pair as a function of stimulus frequency $\Omega$ (abscissa) and relative noise amplitude $\sigma_{r}=$ $\sigma /(1-\mu)$ (ordinate). The color scale for each column is given at the bottom. The black asterisk marks the location of the SNR maximum. Stimuli in the white ..... (continued) 

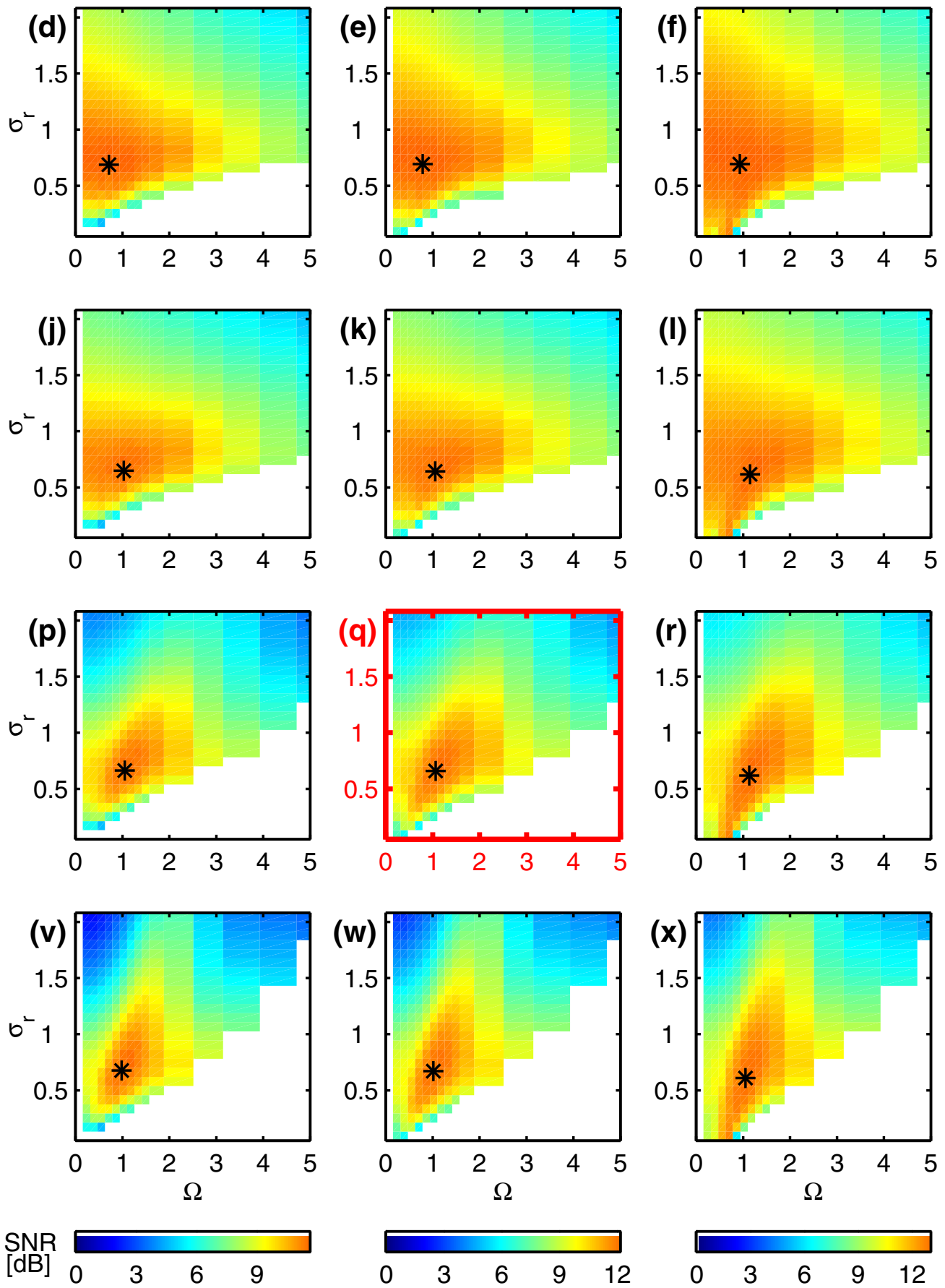

Figure 5.17(continued): . . area to the bottom right of the figures evoked no measurable responses. Each row of the figure represents one value of the base current $\mu=0.6,0.7$, $0.9,0.95$ (top to bottom), and each column to one value of the relative stimulus amplitude $q_{r}=q /(1-\mu)=0.4,0.6,0.8,0.9,1.0,1.2$ (left to right). The standard stimulus of Table 5.1 corresponds to subfigure (q), highlighted in red. 


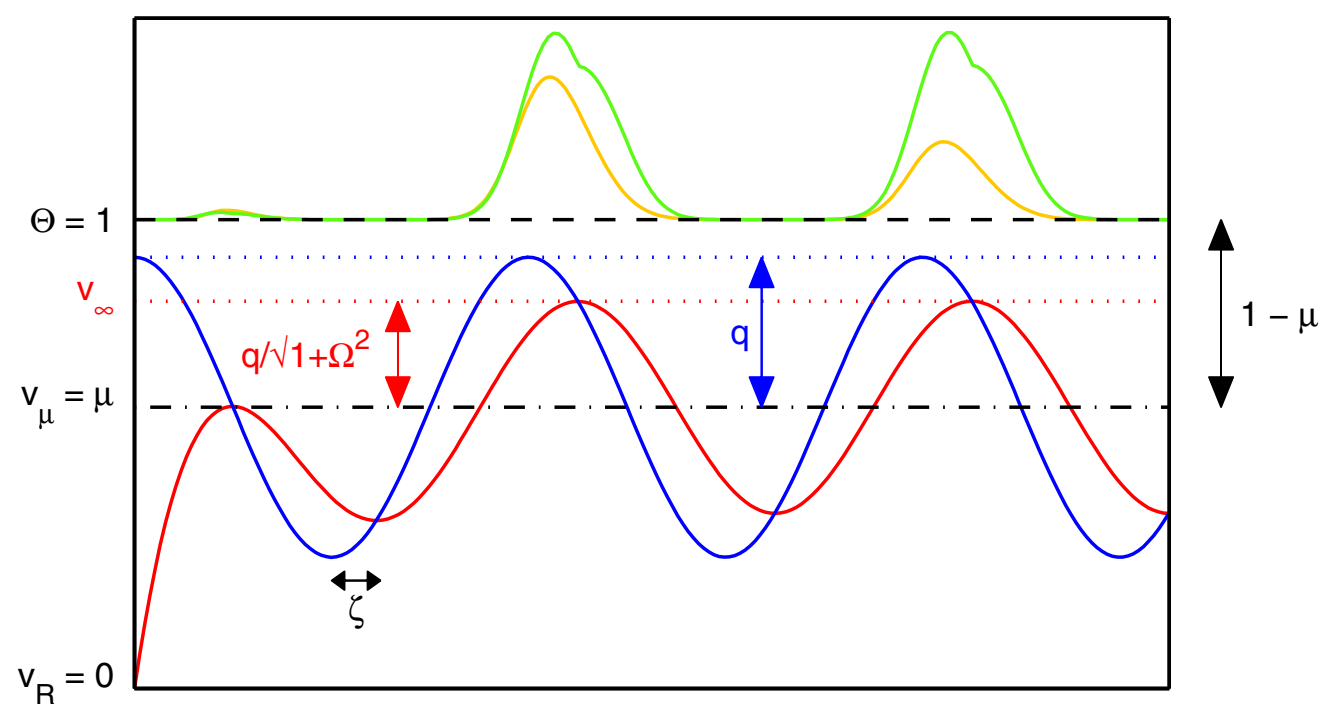

Figure 5.18: The stimulus $I(t)=\mu+q \cos \Omega t$ shown in blue depolarizes the neuronal membrane to the noise-free membrane potential $v_{0}(t)$ drawn in red. After refractory effects have died out, the noise-free potential oscillates about the base potential $v_{\mu}=\mu$ with an amplitude of $q / \sqrt{1+\Omega^{2}} \cdot v_{\infty}$ is the supremum of $v_{0}(t)$ as indicated by the dotted red line and $\zeta$ is the phase lag of $v_{0}(t)$ vs. $I(t)$. Relative amplitudes $q_{r}$ and $\sigma_{r}$ are measured in relation to $1-\mu$, marked by the black arrow to the right. The ISI density (yellow) and Arrhenius\&Current hazard (green) are given for comparison on top for noise amplitude $\sigma=(1-\mu) / 2$.

discussed in terms of relative amplitudes

$$
\sigma_{r}=\frac{\sigma}{1-\mu}, \quad q_{r}=\frac{q}{1-\mu}, \quad \hat{q}_{r}=\frac{q_{r}}{\sqrt{1+\Omega^{2}}},
$$

as illustrated in Fig. 5.18. Figure 5.19 shows how the optimal frequency and noise amplitude depend on the stimulus amplitude $q_{r}$ for different values of $\mu$. The most striking observation is that the optimal signal-to-noise ratio grows linearly with the relative stimulus amplitude and is virtually independent of the base current. Note that the "raw" SNR ${ }^{\text {opt }}$ is plotted in Fig. 5.19(a), while it is given in decibels in all other figures. Perhaps even more surprising is the finding that the optimal relative noise amplitude

$$
\sigma_{r}^{\mathrm{opt}} \approx \frac{2}{3}
$$

is nearly independent of both base current and stimulus amplitude. It appears to be a universal parameter of the system. The optimal frequency, on the other hand, depends roughly linearly on the stimulus amplitude with a slope that decreases with increasing base current.

The Arrhenius\&Current approximation to the Ornstein-Uhlenbeck neuron as introduced in Chapter 3 provides an explanation for these observations. 

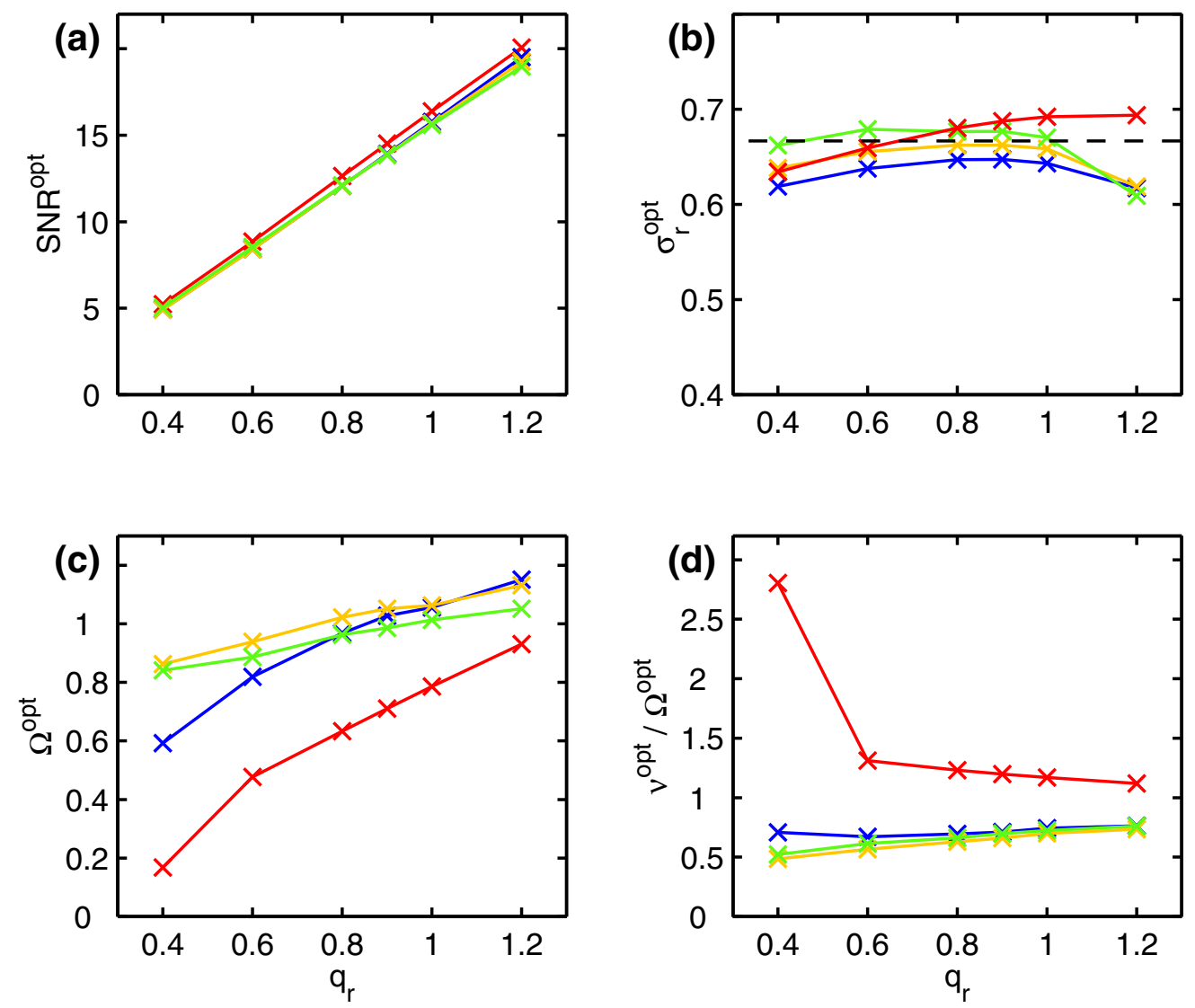

Figure 5.19: (a) The optimal signal-to-noise ratio $\mathrm{SNR}^{\mathrm{opt}}$ depends linearly on the relative stimulus amplitude $q_{r}$ and is virtually independent of the base current $\mu$ for $\mu \in[0.6,1)$, i.e. for refractory coefficients $\gamma>1.5$. Base currents are $\mu=0.6$ (red), 0.7 (blue), 0.9 (yellow) and 0.95 (green). (b) The optimal noise amplitude $\sigma^{\text {opt }} \approx 2 / 3$ (dashed) is almost independent of base current and stimulus amplitude. (c) The slope of the graph relating optimal frequency to relative stimulus amplitude is the higher, the smaller the base current. (d) The ratio of optimal firing rate to stimulus frequency is the average number of spikes per stimulus period. Values larger than one indicate a bursty firing pattern. Crosses mark data points, while lines are to guide the eye.

This approximation provides a good model for the signal-to-noise ratio as is demonstrated in Fig. 5.20. The firing pattern of the A\&C model is determined by its hazard function, whence an analysis of the latter should elucidate all dependencies of interest. With the noise-free potential $v_{0}(t)$ from Eq. (2.43), Eq. (3.8) yields for the hazard

$$
h(t)=\left(w_{1}+w_{2}[Y(t)]_{+}\right) \mathrm{e}^{-x(t)^{2}}
$$

with

$$
x(t)=\frac{1}{\sigma_{r}}\left[1+\gamma \mathrm{e}^{-t}-\hat{q}_{r} f(t)\right], \quad Y(t)=\frac{1}{\sigma_{r}}\left[\gamma \mathrm{e}^{-t}-\hat{q}_{r} g(t)\right]
$$




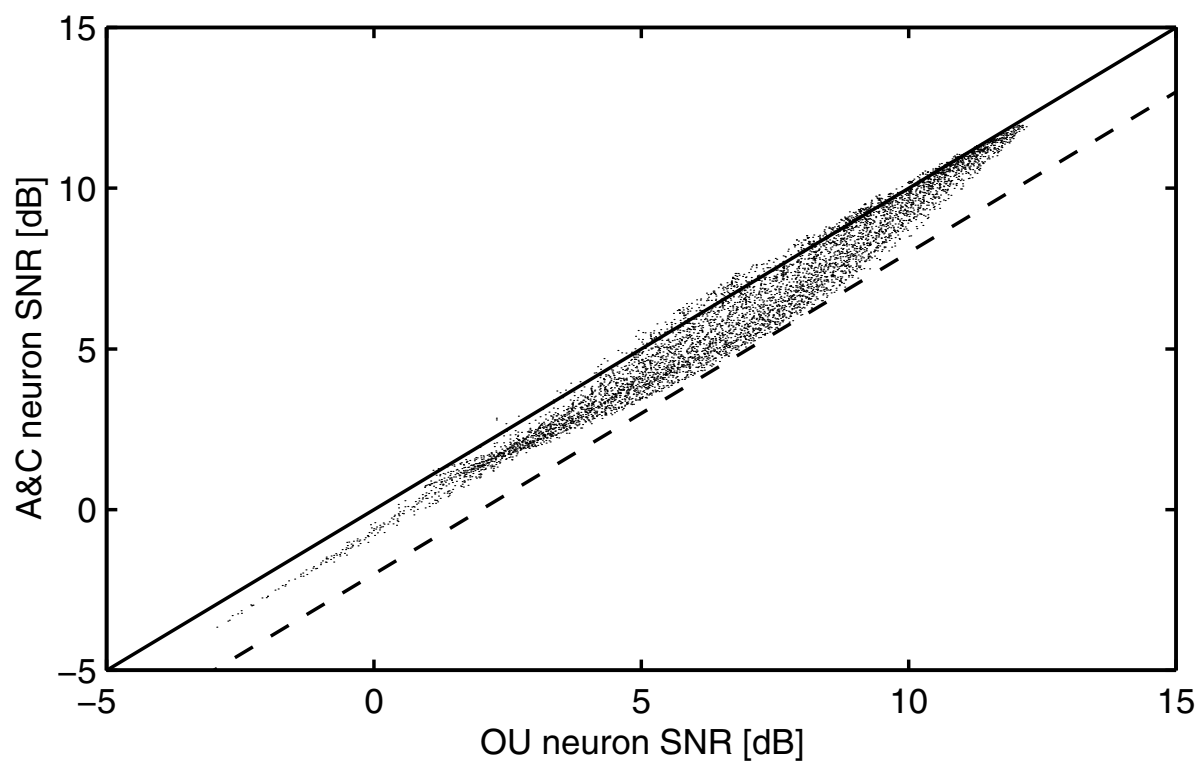

Figure 5.20: The signal-to-noise ratio of spike trains fired by the Ornstein-Uhlenbeck neuron is approximated well by the Arrhenius\&Current model as shown in this scatter plot. Data are from some 6000 parameter combinations of Fig. 5.17. The solid line marks the identity, while the dashed line is at $-2 \mathrm{~dB}$.

and

$$
\begin{aligned}
f(t) & =\cos \left(\Omega t+\phi_{0}-\zeta\right)-\mathrm{e}^{-t} \cos \left(\phi_{0}-\zeta\right) \\
g(t) & =f(t)-\cos \left(\Omega t+\phi_{0}\right), \quad \zeta=\operatorname{atan} \Omega
\end{aligned}
$$

Base current and reset potential determine the refractory coefficient

$$
\gamma=\frac{\mu-v_{R}}{1-\mu}
$$

which simplifies to $\gamma=\mu /(1-\mu)$ in the afferent regime. It will be positive except for the unusual case of hyperpolarizing (negative) base current. The periodic stimulus enters the hazard only through the functions $f(t)$ and $g(t)$ with the common coefficient $\hat{q}_{r}$, while refractoriness is caused by the term $\gamma \mathrm{e}^{-t}$, which captures the mean depolarization towards the base potential $v_{\mu}=\mu$ due to the base current, cf. Fig. 5.18. The refractory term $\mathrm{e}^{-t} \cos \left(\phi_{0}-\zeta\right)$ contained in $f(t)$ will be negligible as long as $\hat{q}_{r} \ll \gamma$.

Both noise and stimulus amplitude enter the hazard only as relative amplitudes, whence the response of the neuron should depend only on $\sigma_{r}$ and $q_{r}$. This is demonstrated for the noise amplitude in Fig. 5.21: graphs of the signal-to-noise ratio vs. noise amplitude for different $\mu$ are strikingly different when the absolute amplitude $\sigma$ is chosen as abscissa, Fig. 5.21(a), but coincide perfectly for $\sigma_{r}<1$ if plotted against the relative amplitude $\sigma_{r}$, Fig. 5.21(c). For strong noise, the signal-to-noise ratio is much larger for small base current than for large. Figs. 5.21(b) and (d) indicate that this deviation arises because 

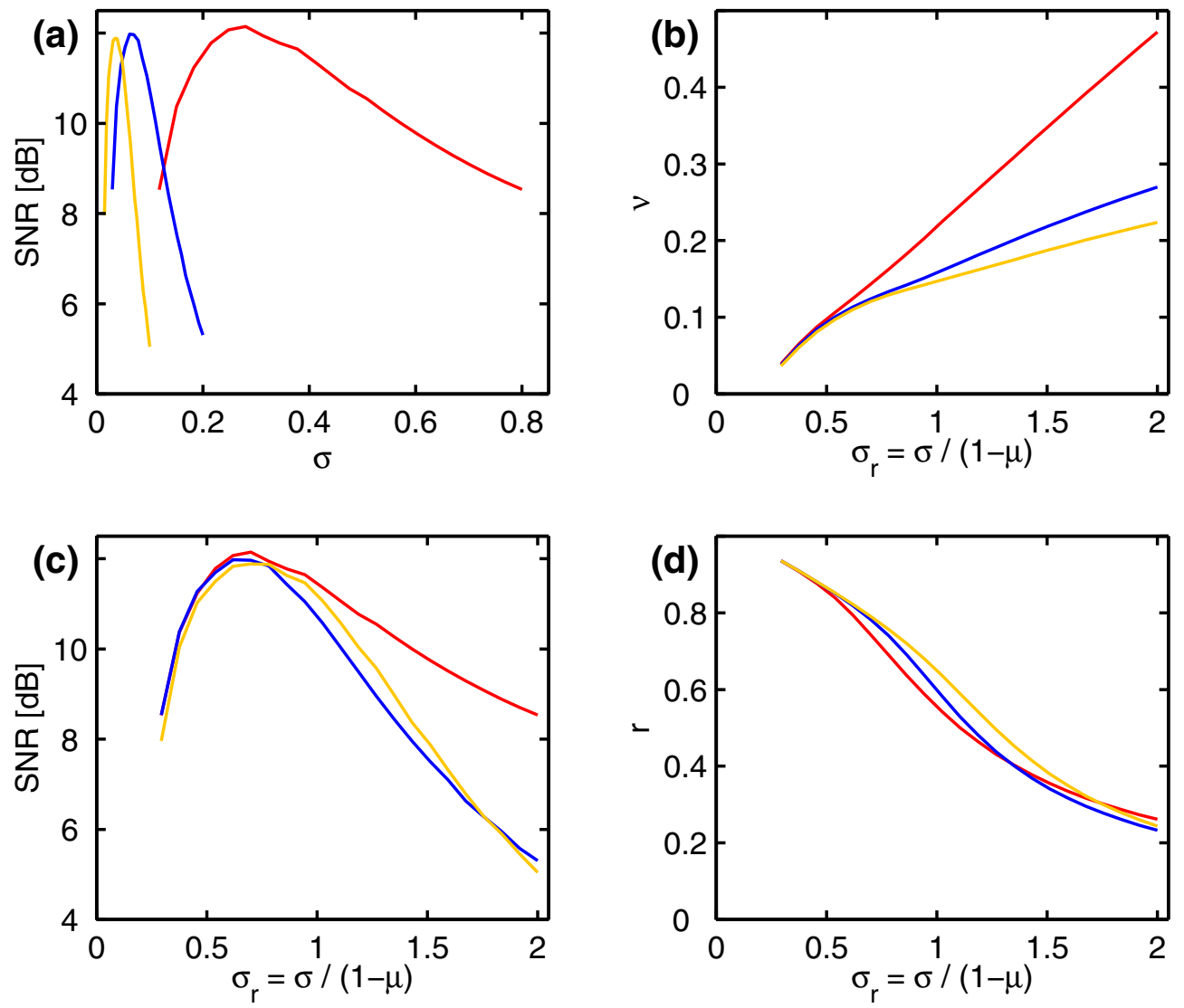

Figure 5.21: The dependence of the signal-to-noise ratio on the absolute noise amplitude $\sigma$ varies strongly with the base current $\mu(\mathrm{a})$, while the dependence on the relative noise amplitude is universal for $\sigma_{r}$ not too large (c). Differences for $\sigma_{r} \gg 1$ are caused by the firing rate (b), while the vector strength differs little (d). Base currents are $\mu=0.6$ (red), 0.9 (blue), and 0.95 (yellow) with refractory coefficients $\gamma=1.5,9$, and 19, respectively. Relative stimulus amplitude is $q_{r}=1$ and frequency $\Omega=0.35 \pi$.

the firing rate increases much faster with noise for small than for large base current $\mu$; the vector strength, in contrast, varies only little with $\mu$. This effect is mediated by the refractory coefficient $\gamma$, which rises from $\gamma=0$ for $\mu=0$ to a divergence at $\mu=1$. Thus, $x(t)$ will be large and hence the hazard $h(t)$ small if base current and refractory coefficient are large, precluding high firing rates. For small $\mu$ and $\gamma$, the converse is true. The paradox that small base currents yield high firing rates is but an apparent one: it arises because stimulus and noise amplitudes are taken relative to $1-\mu$. For fixed absolute amplitudes $q$ and $\sigma$, the firing rate rises with the base current. If noise is weak $\left(\sigma_{r}<1\right)$, $x(t)$ will remain large until the refractory term has decayed almost completely, so that the value of the refractory coefficient has no influence on the firing pattern. Thus the same signal-to-noise ratio is obtained independent of the base current in this case. 

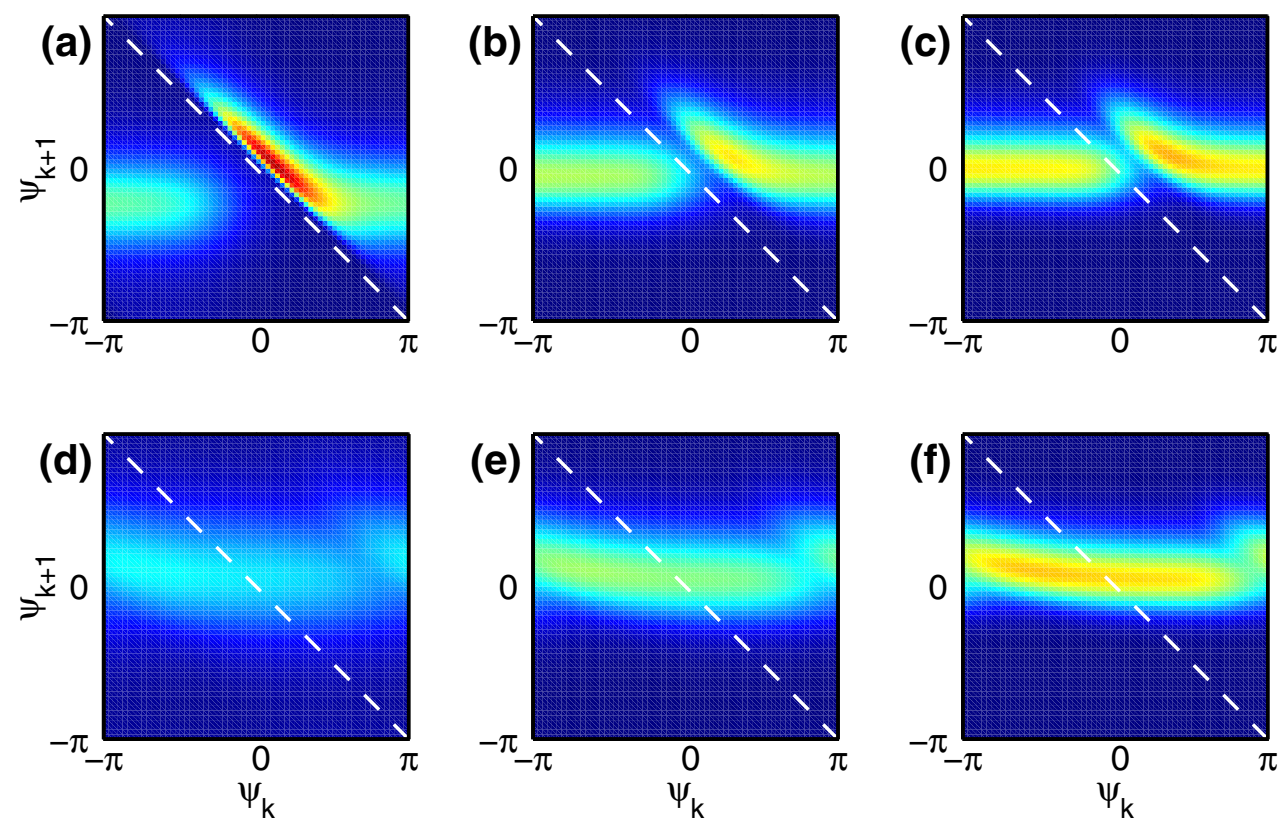

Figure 5.22: Transition matrices at optimal noise-frequency combinations differ depending on base current and stimulus amplitude. Matrices in the top row are for base current $\mu=0.6$ $(\gamma=1.5)$, and for $\mu=0.95(\gamma=19)$ in the bottom row, with relative stimulus amplitudes $q_{r}=0.4,0.8$, and 1.2 from left to right. The color scale ranges from 0 (dark blue) to 0.11 (dark red) for all matrices.

Since the behavior of the neuron is thus independent of the base current for noise not too large, it is only natural that the optimal relative noise amplitude $\sigma_{r}^{\text {opt }}=2 / 3$ is largely independent of the base current. The base current has no influence of the optimal signal-to-noise ratio either, because the hazard will be extremely small until the refractory term has decayed completely if $\sigma_{r}^{\text {opt }}=2 / 3$. This renders $\mathrm{SNR}^{\text {opt }}$ a function of the relative stimulus amplitude $q_{r}$ alone. Why $\sigma_{r}^{\text {opt }}$ is independent of $q_{r}$, remains unclear at present.

The linear dependence of the signal-to-noise ratio on the stimulus amplitude cannot yet be explained, but may be interpreted in the following way. Since $\sigma_{r}^{\text {opt }}$ is constant, the signal-to-noise ratio of the stimulus will be $\mathrm{SNR}_{\text {in }} \sim$ $q_{r}^{2}$ (Wiesenfeld et al. 1994). The observed linearity therefore suggests that $\mathrm{SNR}^{\text {opt }} \sim \sqrt{\mathrm{SNR}_{\text {in }}}$, which is in qualitative agreement with recent results of Burkitt and Clark (1999b) for the relation of input and output vector strength. This would indicate a law of diminishing returns regarding the signal-to-noise ratio of the input, i.e. an upper bound on the useful SNR range of spike trains.

The slope of the graph relating the optimal stimulus frequency $\Omega^{\text {opt }}$ to the relative stimulus amplitude $q_{r}$ is the larger, the smaller the base current $\mu$, see Fig. 5.19(c). A clue to this observation is provided by the ratio of the firing rate to the stimulus frequency, i.e. the average number of spikes per stimulus period, as shown in Fig. 5.19(d). The neuron fires less than one spike per 
stimulus period for large base currents independent of $q_{r}$, indicating phaselocked firing. The optimal frequency depends little on $q_{r}$ in this regime. For small base currents, on the other hand, the neuron achieves the optimal signalto-noise ratio in different ways, depending on the stimulus amplitude: For small $q_{r}$, it prefers slow stimuli, and responds with bursting firing patterns. For large $q_{r}$, it prefers faster stimuli and fires phase-locked single spikes. This analysis is confirmed by the pertaining transition matrices displayed in Fig. 5.22. For small base current ( $\mu=0.6$, top row), the transition from bursting to phaselocked firing is evident. For large base current ( $\mu=0.95$, bottom row), phaselocked firing prevails at all stimulus amplitudes and is merely sharpened with increasing $q_{r}$.

The last paragraph indicates that the neuron may operate in two distinct regimes. For large base currents, i.e. strong average depolarization, the neuron will preferentially transmit stimuli of a fixed frequency, $\Omega^{\text {opt }} \approx 1$. For small base currents, in contrast, the preferred frequency is a function of the stimulus amplitude, whence particular stimulus frequencies may be selected by varying the stimulus amplitude.

\subsubsection{Stochastic resonance in the cortical regime}

All results presented hitherto presumed a reset potential $v_{R}=0$, far below the threshold at $\Theta=1$. While this appears plausible when modeling afferent neurons, Troyer and Miller (1997) have argued that a reset potential of $v_{R}=0.7$ is more reasonable for cortical cells. Figure 5.23 demonstrates stochastic double resonance in this regime for base currents $\mu=0.9$ and 0.95 for some of the stimulus amplitudes used in the afferent case, cf. Fig. 5.17. The optimal signal-to-noise ratio, frequency and noise amplitude are given in Fig. 5.24 together with results for the afferent case. Results are nearly identical except for the optimal frequency, which varies with stimulus amplitude much more in the cortical than the afferent regime, particularly for $\mu=0.9$. This occurs because the smaller reset induces a much shorter refractory time (refractory coefficient $\gamma=2$ instead of $\gamma=9$ in the afferent regime). This permits the neuron to attain a high signal-to-noise ratio by firing sharp bursts at intermediate to low frequencies when driven by stimuli with small amplitudes. That bursting does indeed occur for $\mu=0.9$ and $q_{r}=0.4$ is vindicated by the transition matrix for this parameter combination, see Fig. 5.24(d).

The comparison of afferent and cortical regimes suggests that the optimal signal processing properties will depend on base current and reset potential only through the refractory coefficient. This is obviously true for the Arrhenius\&Current model, since $\mu$ and $v_{R}$ enter the hazard only through $\gamma$. To test if the same holds for the Ornstein-Uhlenbeck neuron, optimal noise amplitudes and frequencies were computed for six values of the refractory coefficient $(2 \leq \gamma \leq 20)$ and three values of the reset potential $\left(v_{R}=0,0.35,0.7\right)$ with 

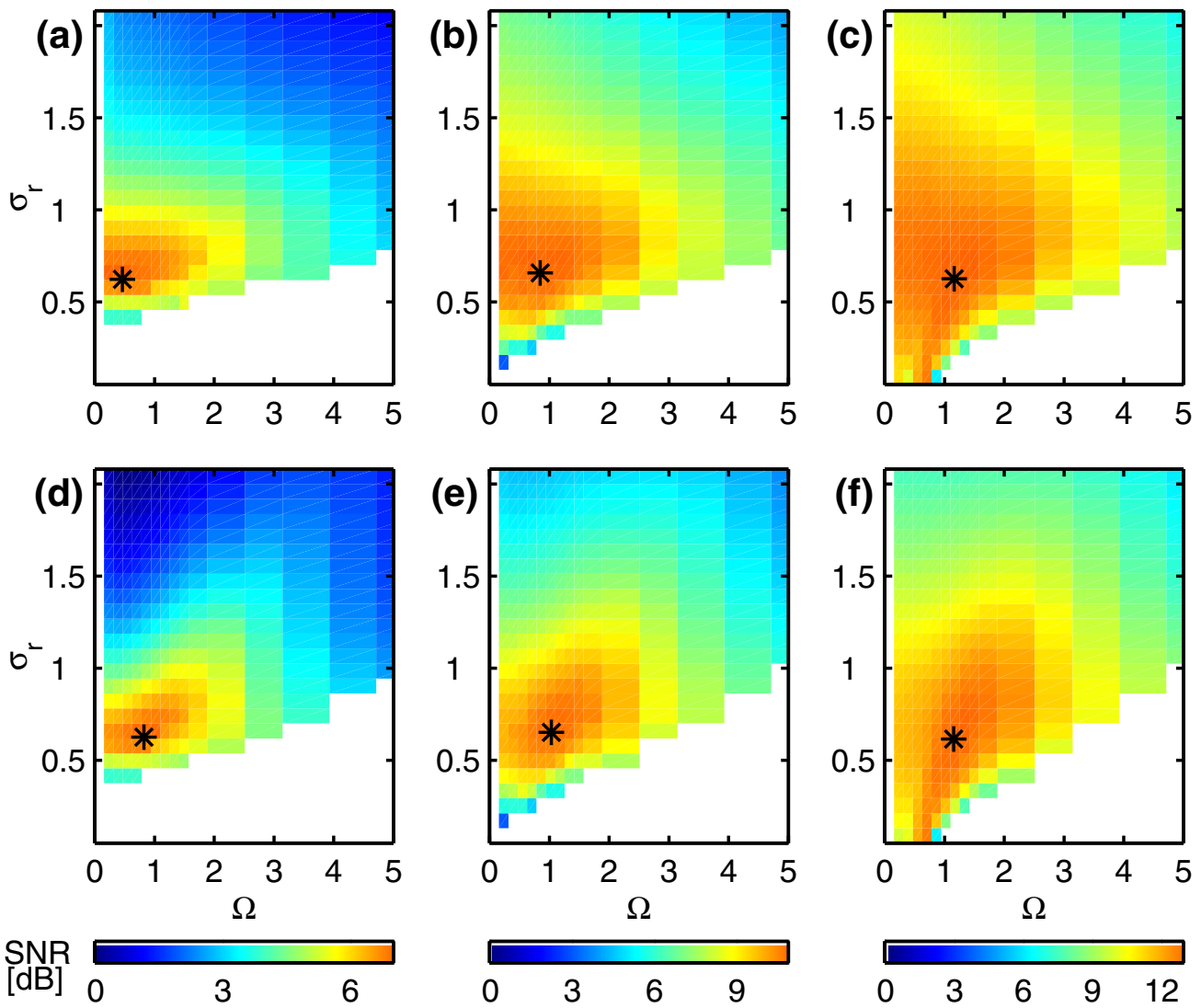

Figure 5.23: Signal-to-noise ratio for base currents $\mu=0.9$ (top) and $\mu=0.95$ (bottom) in the cortical regime, $v_{R}=0.7$. Relative stimulus amplitudes are $q_{r}=0.4,0.8$ and 1.2 from left to right. Within each figure, the stimulus frequency $\Omega$ is on the abscissa, and the relative noise amplitude $\sigma_{r}$ on the ordinate, while colors encode the SNR in decibel. The black asterisk marks the location of the SNR maximum. White at the bottom right of the figures corresponds stimuli evoking no measurable response.

corresponding base currents $\mu$ from Eq. (5.4c). Results are shown in Fig. 5.25 as optimal frequency vs. stimulus amplitude graph and indicate that response properties depend only on $\gamma$, but not on $\mu$ and $v_{R}$ alone. The same holds for the optimal noise amplitude and signal-to-noise ratio (not shown). The relation between optimal frequency $\Omega^{\text {opt }}$ and stimulus amplitude $q_{r}$ is almost perfectly linear for all values of the refractory coefficient. The slope of these linear fits increases with decreasing refractory coefficient, but unfortunately cannot be related to it in a straightforward manner. If refractory effects are strong $(\gamma>4)$, the frequency-noise relation is fairly flat, as the neuron fires phase-locked spike trains independent of stimulus amplitude. For small values of the refractory coefficient, on the other hand $(\gamma<3)$, the optimal frequency depends markedly on the stimulus amplitude as bursting becomes attractive for small amplitudes, as discussed for the afferent regime above. The deviation 

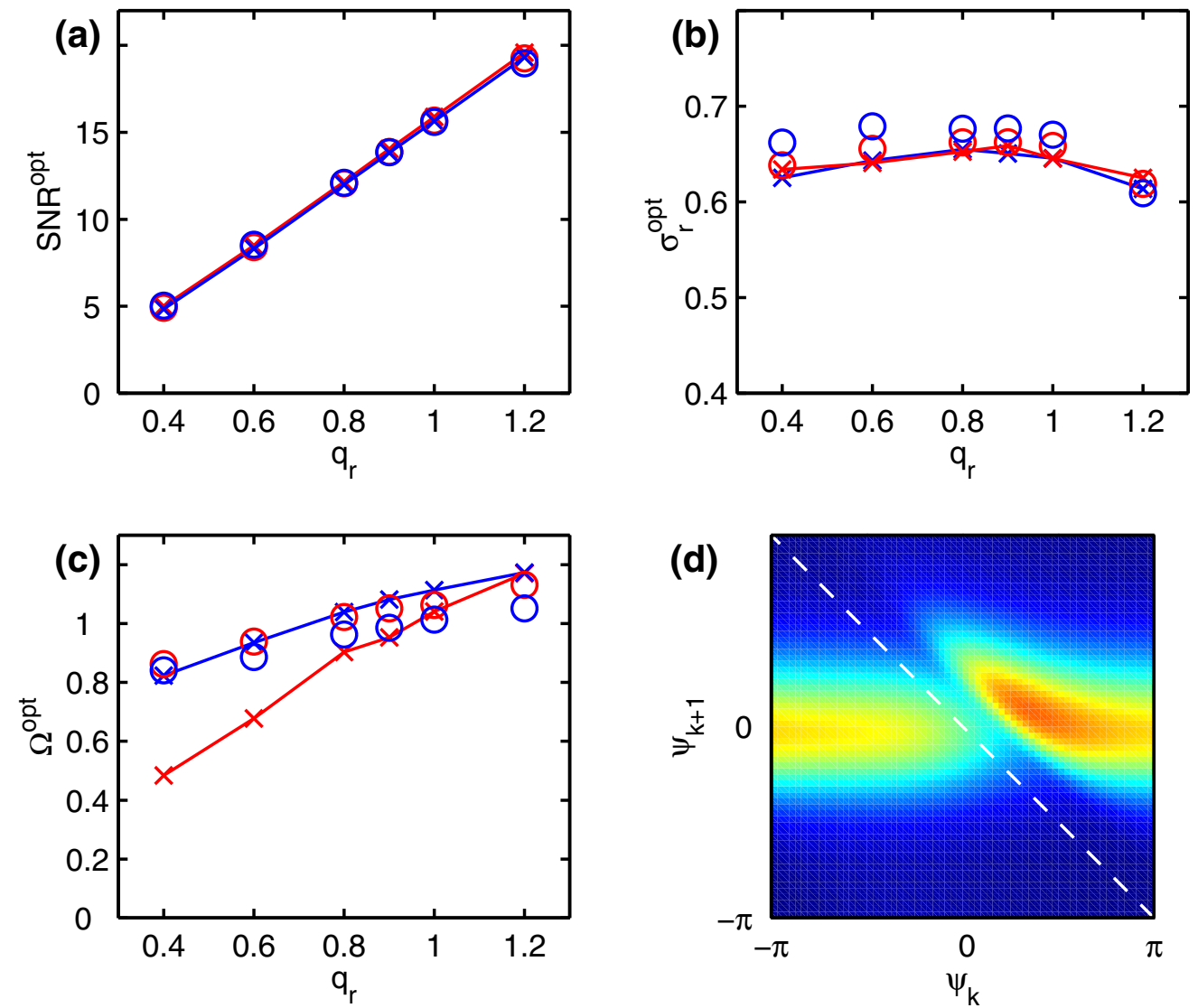

Figure 5.24: (a) The optimal signal-to-noise ratio $\mathrm{SNR}^{\mathrm{opt}}$ in the cortical regime (crosses, reset potential $\left.v_{R}=0.7\right)$ is identical to that in the afferent regime (circles, $v_{R}=0$ ) across a wide range of stimulus amplitudes $q_{r}$ for base currents $\mu=0.9$ (red) and 0.95 (blue). The same holds for the optimal relative noise amplitude $\sigma_{r}^{\text {opt }}$ in (b), while (c) the frequencyamplitude graph is considerably steeper in the cortical than in the afferent regime. (d) The upward-deflected finger in the transition matrix for $v_{R}=0.7, \mu=0.9, q_{r}=0.4$ indicates that a bursting firing pattern is the reason for the low optimal frequency. Lines are to guide the eye.

from linearity for $\gamma=4$ most likely results from the cross-over between weak and strong refractoriness.

This raises the question of what happens if refractory effects become minuscule. If, in particular, $\mu=v_{R}$, the refractory coefficient vanishes. Refractoriness thus enters the hazard function only through the refractory term in $f(t)$, cf. Eq. (5.4), and will be comparatively small, since suprathreshold stimuli with $q_{r} \gg 1$ are not considered here; see Tateno (1998) for a treatment of that regime. If the Ornstein-Uhlenbeck neuron has no refractory time under such conditions, nothing can be gained from matching the stimulus frequency to it, and one should expect highest signal-to-noise ratios at extremely low frequencies, at which the stimulus amplitude is dampened least through inte- 


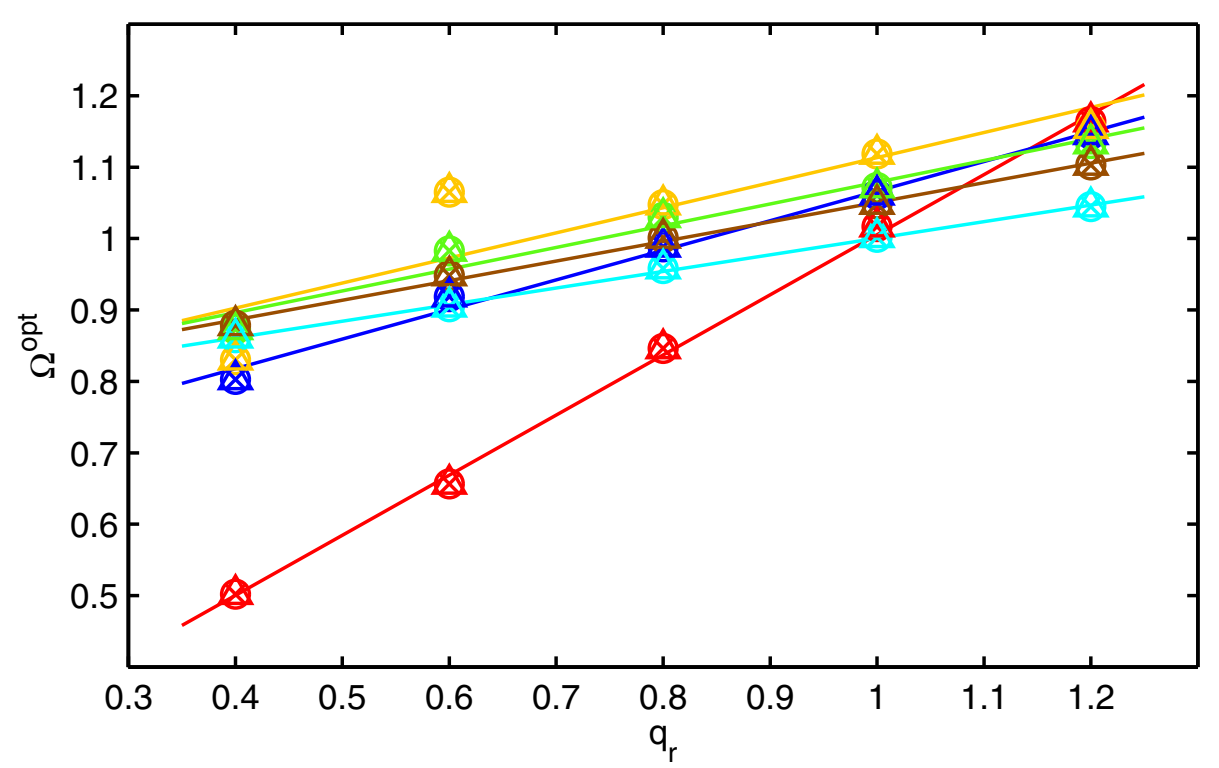

Figure 5.25: The relation between optimal frequency $\Omega^{\text {opt }}$ and stimulus amplitude $q_{r}$ depends on base current $\mu$ and reset potential $v_{R}$ only through the refractory coefficient $\gamma=\left(\mu-v_{R}\right) /(1-\mu)$. Colors mark $\gamma=2$ (red), $\gamma=2.5$ (blue), $\gamma=4$ (yellow), $\gamma=8$ (green), $\gamma=12$ (brown) and $\gamma=20$ (cyan), while circles indicate $v_{R}=0$, crosses $v_{R}=0.35$ and triangles $v_{R}=0.7$. Base currents range from $\mu=0.666$ to $\mu=0.986$. Solid lines are linear least-squares fits.

gration. Preliminary evidence suggests that this is indeed the case. But it is likely that the Ornstein-Uhlenbeck neuron has to be modified slightly in this "non-refractory" regime, for it is known from neurophysiological experiments that neurons possess an absolute refractory time of a few milliseconds (Kandel et al. 1991), during which no spikes can be generated at all. As long as the relative refractory time required to depolarize the neuron to the base potential $v_{\mu}=\mu$ after each spike/reset is long compared to the absolute refractory time, there is little need to explicitly introduce the latter into the model. If relative refractoriness, on the other hand, does no longer keep the neuron from firing at too short intervals, absolute refractory mechanisms need to be modeled. A definite answer will only be available after further studies, but a prediction is possible: stochastic double resonance will arise through proper matching of the stimulus timescale to the absolute refractory time. 


\section{Chapter 6}

\section{Summary}

Stochastic double resonance has firmly been established as a property of the Ornstein-Uhlenbeck neuron. This result is related to the existing literature below, and its consequences for neuronal signal processing are discussed. Prior to this, the methodological advances presented in this thesis and the results for the case of constrained stimulation are reviewed briefly.

\section{Methodological achievements}

The most important technical contribution of this thesis is the Markov chain analysis of the neuronal response to unconstrained periodic stimulation. It provides explicit expressions for the stationary spike phase, interspike-interval, and power spectral densities of spike trains. The Markov chain in time permits for the computation of the spike intensity, thus facilitating the analysis of responses to time-dependent stimuli. The precise relation of the spike intensity to the population activity of neural networks studied by Gerstner (1999a) remains to be clarified. Since the Markov chains both in time and in phase are applicable to arbitrary Markov point processes, they should find applications in many fields of research (Burkitt and Clark 1999b).

The analysis of responses to constrained stimulation was largely based on standard techniques of renewal theory dating back to Lukes (1961). The spike intensity was fruitfully exploited to demonstrate the temporal structure of responses to constrained stimulation.

The algorithm for the computation of interspike-interval densities of the Ornstein-Uhlenbeck neuron with time-dependent stimulation should prove useful to research involving the first-passage time problem of forced OrnsteinUhlenbeck processes in general. It may indeed be applied to any kind of escape process as long as the distribution of the state variable in the absence of the absorbing boundary is known - it simply replaces the Gaussians in Schrödinger's renewal equation. This generality, the transparency of the Ansatz, and the greater numerical stability make it preferable to the method of Buonocore 
et al. (1987). As an aside, Schrödinger's Ansatz was instrumental to prove the strict positivity of the interspike-interval density, which in turn allowed to establish the convergence of the Markov chain to a stable stationary phase distribution. The Ansatz has further been exploited as a starting point for a perturbation analysis of synchrony in neuronal firing (Burkitt and Clark 1999a). A comparison of perturbation and exact results is currently underway.

The Arrhenius\&Current model provides for the first time a validated approximation to the dynamics of the Ornstein-Uhlenbeck neuron. The Arrhenius and Abeles models were found to be slightly less accurate, but sufficient to be of use in analytical studies demanding utmost mathematical simplicity. Since these approximations pertain to the first-passage time problem of the temporally modulated Ornstein-Uhlenbeck process, which has eluded analytical solution throughout a half-century of research, they should meet with widespread interest.

Answers have been provided here for all open issues listed by Gammaitoni et al. (1998) as quoted in the introduction: first-passage-time distributions are treated for periodic forcing without reset; reliable approximations to these distributions were developed and tested; and the lower bound on the membrane potential fluctuations was shown to be irrelevant to the response properties of sensory afferent neurons in two mammalian species. Program code for the algorithms developed here will be provided publicly as a service to the scientific community (Plesser 1999).

\section{Stochastic resonance under constrained stimulation}

Classic stochastic resonance has been demonstrated for the Ornstein-Uhlenbeck neuron driven by a constrained sinusoidal stimulus. The signal-to-noise ratio is maximized if the period of the non-sinusoidal effective stimulus, which results from the reset after each spike, matches the frequency dominating the power spectrum of the spike train. This frequency matching arises, because the modes of the interspike-interval distribution, which are located near multiples of the stimulus period, shift towards shorter times with increasing noise. The stochastic resonance effect was shown to disappear if the initial phase, to which the stimulus is reset, was adapted to the noise amplitude in such a way that the neuron would most likely fire at this initial phase during a later period, effectively eliminating the stimulus reset. Therefore, stochastic resonance is an artifact of the fixed reset phase and not likely to be relevant to biology - the model rather reminds of a neuron stimulated by an old record with a crack.

The spectral analysis presented by Plesser and Tanaka (1997), including the definition of the signal-to-noise ratio of constrained spike trains, were taken up by Shimokawa et al. (1999a), who focused on stochastic resonance defined in terms of the interspike-interval distribution along the lines of Bulsara et al. (1996). The effects they observed occur in the presence of strong noise (ampli- 
tudes $\sigma>0.1$, compared to $\sigma \ll 0.1$ in the constrained regime here) and were accompanied by signal-to-noise ratios close to zero decibel (Shimokawa et al. 1999a, Fig. 19(b)). This regime seems of little relevance to signal processing.

\section{Stochastic Double Resonance}

The preeminent finding of this thesis is that the Ornstein-Uhlenbeck neuron benefits from noise twofold when encoding periodic stimuli into spike trains. Not only is there a particular noise amplitude providing for a maximal signalto-noise ratio of the output, but there is a preferred range of frequencies as well. I propose to call this effect stochastic double resonance.

The neuron will thus encode stimuli of some frequencies as spike trains that are both intense and have a precise temporal structure. Neurons at the next processing stage will easily discern these regular trains from a homogeneous background of activity. Frequencies outside the preferred band, in contrast, elicit irregular firing patterns, which will appear to its recipients to be little more than - noise! To put it differently: The presence of noise permits the neuron to operate as a synchronization bandpass filter in the subthreshold regime. This bandpass can operate in two distinct fashions, which are easily switched. The transmission mode is activated by a large base current, which renders the preferred frequency of the neuron nearly independent of the stimulus amplitude. Signals within the optimal band will thus be transmitted regardless of their amplitude. Small base currents provide for the discrimination mode, as the preferred frequency depends linearly on the stimulus amplitude. This opens a way to selectively gate signals based on their amplitude: Assume that a periodic stimulus of a given frequency impinges on the dendrite of the neuron. Other input to the dendritic tree might vary the amplitude of the resulting periodic input current on its way to the soma and thus tune the preferred frequency to that of the stimulus or away from it.

It is most remarkable that the optimal signal-to-noise ratio is practically independent of the stimulus amplitude and merely depends linearly on the base current. The noise level may thus be fixed independent of the processed signals, making the processing scheme suggested above feasible. The value $\sigma_{r}^{\text {opt }} \approx 2 / 3$ of the optimal relative noise amplitude is in good agreement with the finding by Kempter et al. (1998) that neuronal coincidence detectors operate best if the base potential is roughly one noise amplitude below threshold, i.e. $\sigma_{r} \approx 1$.

The range of optimal frequencies in the discrimination mode spans roughly one octave. The absolute value of the optimal frequency depends on the membrane time constant $\tau_{m}$ of the neuron under consideration. The latter might be as low as $200 \mu$ s for specialized afferent neurons, but reach $10 \mathrm{~ms}$ in cortical neurons. Signal processing might thus benefit from stochastic double resonance throughout entire range of frequencies observed in the central nervous system, from slow theta-waves of the EEG to phase-locked responses in the kilohertz range in the auditory pathway. 


\section{Related work and generalization}

A recent series of publications suggests that stochastic double resonance is not a peculiarity of the Ornstein-Uhlenbeck neuron, but a phenomenon nearly as universal as classic stochastic resonance. Berdichevsky and Gitterman (1996) found a resonance with respect to stimulus frequency in noise-induced hopping in a piecewise constant bistable potential - a rather unphysiological system. Liu et al. (1999) and Kanamaru et al. (1999) have since demonstrated stochastic double resonance in the noisy Hindemarsh-Rose and FitzHughNagumo neuron models, respectively. That a model as simple as the OrnsteinUhlenbeck neuron exhibits the same stochastic double resonance effects found in these complex nonlinear models of neuronal membrane dynamics once again suggests the use of this simpler model. Indeed, the simplicity of the model allowed for a detailed and rewarding analysis of the parameter dependencies of stochastic double resonance, while the former studies were based on simulations. Simplification must not be carried too far: stochastic resonance in stimulus frequency can occur only in systems which have a timescale that is to be matched. Response properties of level crossing detectors, e.g., which generate an output spike every time the membrane potential crosses the threshold in upward direction, but do not influence the potential in any way, are independent of the signal frequency for lack of an internal timescale (Gingl et al. 1995).

Gammaitoni's original demonstration of stochastic resonance in frequency (1995) — or bona fide resonance as he called it - has been criticized on methodological grounds by Choi et al. (1998), but a new experimental report in the spirit of Gammaitoni avoids the pitfalls (Giacomelli et al. 1999). It thus provides clear-cut evidence that stochastic double resonance is indeed a physical and not just a mathematical phenomenon.

Shimokawa et al. (1999b) recently presented a closely related study on stochastic resonance in periodically driven Ornstein-Uhlenbeck neurons. They employ the same Markov chain method as developed independently in this thesis to determine the stationary phase density. Instead of computing the power spectrum directly from the transition matrix as suggested here, they resort to an ensemble of identical, independent neurons. The superposition of the spike trains fired by all these neurons is an inhomogeneous Poisson process with a time-dependent rate. The rate modulation within each stimulus period is given by the stationary phase density in this case. This yields precisely the model expression SNR $\sim \nu r^{2}$ for the signal-to-noise ratio. Results as reported by Shimokawa et al. (1999b) are in agreement with those presented here, but more limited. The authors do not observe stochastic double resonance, most likely because they adapt the stimulus amplitude such that $q / \sqrt{1+\Omega^{2}}$ is held constant for all frequencies. 
Stemmler (1996) concluded that different "flavors" of stochastic resonance are intimately related. In particular, he showed that the probability of correct detection of a constant signal, the mutual information between an aperiodic signal and the spike train it elicits, and the signal-to-noise ratio of a spike train elicited by sinusoidal stimulation can all be expressed in terms of the lower bound of the Fisher information (Cover and Thomas 1991). Thus one may surmise that the results derived here for sinusoidal stimulation will at least qualitatively hold for aperiodic signals as well, and are indeed ubiquitous.

\section{Perspectives: Neurocomputing with noise}

Noise endows neurons with the capability to selectively transmit stimuli in spike trains of varying regularity. This may contribute to neural information processing as follows. Each pathway of processing has a small number, or pool, of neurons at each stage, all conveying essentially the same signal. A neuron will project its output to many neurons in the corresponding pool of the subsequent stage, while receiving input from a large number of neurons in the antecendent pool. Besides input from within the chain of pools, each neuron receives a tremendous number of uncorrelated inputs: proportions might easily reach 100 neurons in a pool compared to 10000 uncorrelated inputs. Such chains of neuron pools are known as synfire chains (Abeles 1991; Herrmann et al. 1995). Pulse packets - barrages of spikes - can propagate through a chain provided they are sufficiently strong and "tight", i.e. synchronized (Diesmann et al. 1999). If the synchronization of output spikes can be varied by modulation of the input spike amplitudes - or rather of the amplitudes of the effective current fluctuations at the soma-pulse packets may be gated on their way through the processing pathways by de- and re-synchronization. Indeed, Aertsen et al. (1989) and Boven and Aertsen (1990) concluded from simulation studies that the "effective connectivity" of neurons is modulated by uncorrelated background activity in a neural network. Stochastic double resonance as demonstrated here might thus prove to be the key to neurocomputing with synfire chains.

Stochastic resonance is sometimes criticized for not being perfect (DeWeese and Bialek 1995; Galdi et al. 1998; Tougaard 1999): optimal signal detectors will do better without noise than devices based on stochastic resonance. But this comes at a price - a control mechanism is required to adapt the detector to the task at hand. Engineers might be willing to pay the price, nature most certainly is not, given the extravagant constraints on building a brain: The brain must be able to build itself from the scant blueprint provided by the genes; it should function reliably over the course of a century, with facilities to compensate all but the most severe injuries; and it has to receive, analyze, store, retrieve, compare and emit signals all at once; above all, it has to be portable and consume as little energy as possible. If we were not carrying a 
brain on our shoulders day after day, we would steadfastly deny its existence: The conditions listed just cannot be fulfilled by any feat of ingenuity - without noise, that is. A decade of intense research on stochastic resonance, to which this thesis is but a modest contribution, has shown that noise can help to forge the required compromises as it provides for sub-optimal, but highly efficient signal processors. It is thus that noise aids neural information processing. 


\section{Appendix A}

\section{Numerical Evaluation of First-Passage-Time Densities}

Schrödinger's renewal Ansatz, Eq. (2.36), reduces the computation of the firstpassage-time density of the Ornstein-Uhlenbeck process to the numerical solution of the Abel integral equation

$$
g(t)=\int_{0}^{t} \frac{K(t, s)}{\sqrt{t-s}} F(s) \mathrm{d} s
$$

The inhomogeneity $g(t)$ and the kernel $K(t, s)$ are as defined by Eqs. (2.34) and (2.35). The existence of a unique solution $F(t)$ is assured, see Chapter 2.2.

Below, a block-by-block method for the computation of $F(s)$ is presented, which is a corrected form of the method given in Chapter 10.3 of Linz (1985). It is validated in Section A.2.

\section{A.1 Algorithm}

Starting from a known initial point $F_{0}=F(t=0)$, the block-by-block method iteratively computes the solution $F_{j}=F\left(t_{j}\right)$ at equidistant points $t_{j}=j h$ in blocks of two points. Let $F_{0}, F_{1}, \ldots, F_{2 m}$ be known, so that $F_{2 m+1}$ and $F_{2 m+2}$ are to be computed as next block. Then, Eq. (A.1) can be written as

$$
g_{2 m+\ell}=\int_{0}^{t_{2 m+\ell}} \frac{K\left(t_{2 m+\ell}, s\right) F(s)}{\sqrt{t_{2 m+\ell}-s}} \mathrm{~d} s=I_{2 m, \ell}+\int_{t_{2 m}}^{t_{2 m+\ell}} \frac{K\left(t_{2 m+\ell}, s\right) F(s)}{\sqrt{t_{2 m+\ell}-s}} \mathrm{~d} s \text {, }
$$

with $\ell=1,2$. The first term on the right-hand side contains only the known portion of $F(t)$ for $s<t_{2 m}$. Dividing the range of integration into $m$ intervals of length $2 h$ and interpolating $K(t, s) F(s)$ quadratically within each interval, 
one obtains after some algebra

$$
\begin{aligned}
I_{2 m, \ell} & =\int_{0}^{t_{2 m}} \frac{K\left(t_{2 m+\ell}, s\right) F(s)}{\sqrt{t_{2 m+\ell}-s}} F(s) \mathrm{d} s=\sum_{j=0}^{m-1} \int_{0}^{2 h} \frac{y_{m, \ell, j}(s)}{\sqrt{t_{2 m+\ell}-t_{2 j}-s}} \mathrm{~d} s \\
& =\sum_{k=0}^{2 m} w_{m, \ell, k} K_{2 m+\ell, k} F_{k} .
\end{aligned}
$$

Here, $K_{n, k}=K\left(t_{n}, t_{k}\right)$, and

$$
\begin{aligned}
y_{m, \ell, j}(s)= & \frac{1}{2}(s-h)(s-2 h) K_{2 m+\ell, 2 j} F_{2 j} \\
& -s(s-2 h) K_{2 m+\ell, 2 j+1} F_{2 j+1} \\
& +\frac{1}{2}(s-h)(s-2 h) K_{2 m+\ell, 2 j+2} F_{2 j+2} .
\end{aligned}
$$

This leads to the integration weights ${ }^{1}$

$$
w_{m, \ell, k}= \begin{cases}\alpha_{2 m+\ell}(h) & k=0 \\ \alpha_{2 m+\ell-k}(h)+\gamma_{2 m+\ell-k+2}(h) & k=2,4, \ldots, 2 m-2 \\ \gamma_{\ell+2}(h) & k=2 m \\ \beta_{2 m+\ell-k+1}(h) & k=1,3, \ldots, 2 m-1\end{cases}
$$

where

$$
\begin{aligned}
& \alpha_{n}(z)=\frac{\sqrt{z}}{2} \int_{0}^{2} \frac{(1-x)(2-x)}{\sqrt{n-x}} \mathrm{~d} x \\
& \beta_{n}(z)=\sqrt{z} \int_{0}^{2} \frac{x(2-x)}{\sqrt{n-x}} \mathrm{~d} x \\
& \gamma_{n}(z)=\frac{\sqrt{z}}{2} \int_{0}^{2} \frac{x(x-1)}{\sqrt{n-x}} \mathrm{~d} x
\end{aligned}
$$

The integral over the unknown portion of the function in Eq. (A.2), i.e. over $\left[t_{2 m}, t_{2 m+\ell}\right]$, is evaluated in the same manner. For $\ell=1$, the value of $F(s)$ at the interval midpoint $t_{2 m+\frac{1}{2}}$ is obtained by quadratic interpolation

$$
F_{2 m+\frac{1}{2}}=\frac{3}{8} F_{2 m}+\frac{3}{4} F_{2 m+1}-\frac{1}{8} F_{2 m+2} .
$$

Inserting into Eq. (A.2), one obtains two linear equations for the desired func-

\footnotetext{
${ }^{1}$ In the corresponding equation (8.35) in Linz (1985), p. 137, the different cases are not sufficiently separated. This is corrected here.
} 
tion values $F_{2 m+1}$ and $F_{2 m+2}$

$$
\begin{aligned}
g_{2 m+1}-I_{2 m, 1}= & \alpha_{1}\left(\frac{h}{2}\right) K_{2 m+1,2 m} F_{2 m} \\
+ & \beta_{1}\left(\frac{h}{2}\right) K_{2 m+1,2 m+\frac{1}{2}}\left[\frac{3}{8} F_{2 m}+\frac{3}{4} F_{2 m+1}-\frac{1}{8} F_{2 m+2}\right] \\
+ & \gamma_{1}\left(\frac{h}{2}\right) K_{2 m+1,2 m+1} F_{2 m+1}, \\
g_{2 m+2}-I_{2 m, 2}= & \alpha_{2}(h) K_{2 m+2,2 m} F_{2 m} \\
+ & \beta_{2}(h) K_{2 m+2,2 m+1} F_{2 m+1} \\
+ & \gamma_{2}(h) K_{2 m+2,2 m+2} F_{2 m+2} .
\end{aligned}
$$

Solving for the unknowns yields the iteration rule $(m \geq 1)$

$$
\begin{aligned}
& F_{2 m+1}=\frac{1}{\Gamma}\left[\frac{1}{6} K_{2 m+2,2 m+2} S_{1}+\frac{1}{36 \sqrt{2}} K_{2 m+1,2 m+\frac{1}{2}} S_{2}\right] \\
& F_{2 m+2}=\frac{1}{\Gamma}\left[-\frac{2}{9} K_{2 m+2,2 m+1} S_{1}+\frac{1}{6 \sqrt{2}}\left(K_{2 m+1,2 m+\frac{1}{2}}+K_{2 m+1,2 m+1}\right) S_{2}\right]
\end{aligned}
$$

where

$$
\begin{aligned}
\Gamma & =\left(K_{2 m+1,2 m+\frac{1}{2}}+K_{2 m+1,2 m+1}\right) K_{2 m+2,2 m+2}+\frac{2}{9} K_{2 m+1,2 m+\frac{1}{2}} K_{2 m+2,2 m+1}, \\
S_{1} & =\frac{15}{2 \sqrt{h}} g_{2 m+1}-\tilde{I}_{2 m+1}-\left(K_{2 m+1,2 m}+3 K_{2 m+1,2 m+\frac{1}{2}}\right) F_{2 m}, \\
S_{2} & =\frac{15}{2 \sqrt{h}} g_{2 m+2}-\tilde{I}_{2 m+2}-\sqrt{2} K_{2 m+2,2 m} F_{2 m}, \\
\tilde{I}_{2 m, \ell} & =\sum_{k=0}^{2 m} \tilde{w}_{m, \ell, k} K_{2 m+\ell, k} F_{k} \quad, \quad \ell=1,2, \\
\tilde{w}_{m, \ell, k} & = \begin{cases}\tilde{\alpha}_{2 m+\ell} & k=0 \\
\tilde{\alpha}_{2 m+\ell-k}+\tilde{\gamma}_{2 m+\ell-k} & k=2,4, \ldots, 2 m-2 \\
\tilde{\gamma}_{\ell} & k=2 m \\
\tilde{\beta}_{2 m+\ell-k} & k=1,3, \ldots, 2 m-1 .\end{cases}
\end{aligned}
$$

Finally

$$
\begin{aligned}
& \tilde{\alpha}_{n}=\frac{15}{2 \sqrt{h}} \alpha_{n}(h)=-(4 n-3)(n-2)^{\frac{3}{2}}+\left(4 n^{2}-15 n+15\right) \sqrt{n}, \\
& \tilde{\beta}_{n}=\frac{15}{2 \sqrt{h}} \beta_{n+1}(h)=(8 n+12)(n-1)^{\frac{3}{2}}-(8 n-12)(n+1)^{\frac{3}{2}}, \\
& \tilde{\gamma}_{n}=\frac{15}{2 \sqrt{h}} \gamma_{n+2}(h)=(4 n+3)(n+2)^{\frac{3}{2}}-\left(4 n^{2}+15 n+15\right) \sqrt{n},
\end{aligned}
$$

with $\alpha, \beta$, and $\gamma$ from Eq. (A.5).

The precision of the algorithm is limited by that of the coefficients given in Eq. (A.8). While each of the two summands in $\tilde{\alpha}_{n}, \tilde{\beta}_{n}$, and $\tilde{\gamma}_{n}$ are $\sim O\left(n^{5 / 2}\right)$, 


\begin{tabular}{lllllll}
\hline$h$ & $1.0 \cdot 10^{0}$ & $2.0 \cdot 10^{-1}$ & $1.0 \cdot 10^{-1}$ & $1.0 \cdot 10^{-2}$ & $1.0 \cdot 10^{-3}$ & $4.0 \cdot 10^{-4}$ \\
\hline$\Delta_{r}$ & $3.8 \cdot 10^{-2}$ & $5.0 \cdot 10^{-4}$ & $7.3 \cdot 10^{-5}$ & $8.2 \cdot 10^{-8}$ & $8.2 \cdot 10^{-11}$ & $5.2 \cdot 10^{-12}$ \\
$t_{\mathrm{CPU}[\mathrm{s}]}$ & & & & $4.3 \cdot 10^{-1}$ & $4.2 \cdot 10^{1}$ & $2.5 \cdot 10^{2}$ \\
\hline
\end{tabular}

Table A.1: Error $\Delta_{r}$ of the block-by-block method for constant input vs. analytical solution of Eq. (A.10) for different step sizes $h$. The bottom line gives the required CPU time in seconds, with empty fields indicating $t_{\mathrm{CPU}}<0.1 \mathrm{~s}$. The noise amplitude was $\sigma=0.1$ and $0 \leq t \leq 20$. All computations were performed on a COMPAQ XP1000 workstation.

their differences are only $\sim O(1 / \sqrt{n})$. Using standard floating-point arithmetic with 16-digit precision, the coefficients will have only five significant digits. Therefore, the coefficients have been tabulated to 20 significant digits using the MAPLE computer algebra package (Plesser 1999).

The block-by-block algorithm requires $4 m+8$ kernel evaluations to compute points $F_{2 m+1}$ and $F_{2 m+2}$ of the solution. The number of kernel evaluations are required to compute the solution at $M$ points thus grows $\sim M^{2}$. Due to the complicated structure of the weights and the recursive nature of the algorithm, no detailed error analysis is known for this method (Linz 1985, Ch. 10.4).

This algorithm has been implemented for general inhomogeneity $g(t)$ and kernel $K(t, s)$ as program abel. The code has been designed to be as clear as possible. For the Ornstein-Uhlenbeck process, optimized versions have been developed for both periodic (p_fptd) and aperiodic (ap_fptd) stimuli. These implementations enforce non-negative solutions $F$ to improve numerical stability. Source code for both programs is available in electronic form (Plesser 1999).

\section{A.2 Validation}

\section{Constant input}

The first-passage-time density of the Ornstein-Uhlenbeck process for input of the form

$$
I(t)=1+2 c \mathrm{e}^{t}
$$

is given by

$$
\rho(t)=\frac{2 \mathrm{e}^{2 t}}{\sqrt{\pi \sigma^{2}}\left(\mathrm{e}^{2 t}-1\right)^{\frac{3}{2}}} \exp \left[-\frac{\left(1+c-c \mathrm{e}^{2 t}\right)^{2}}{\sigma^{2}\left(\mathrm{e}^{2 t}-1\right)}\right] .
$$

Here, $\sigma$ is the input noise level, the reset voltage is $v(t=0)=v_{R}=0$ and the absorbing threshold is at $v=1$; see Chapter 3.2. For $c=0$, this FPTD 


\begin{tabular}{lllllll}
\hline$h$ & $2.0 \cdot 10^{-1}$ & $1.0 \cdot 10^{-1}$ & $4.0 \cdot 10^{-2}$ & $2.0 \cdot 10^{-2}$ & $1.0 \cdot 10^{-2}$ & $4.0 \cdot 10^{-3}$ \\
\hline (a) & $2.3 \cdot 10^{-4}$ & $4.4 \cdot 10^{-5}$ & $3.3 \cdot 10^{-6}$ & $4.2 \cdot 10^{-7}$ & $5.2 \cdot 10^{-8}$ & $3.3 \cdot 10^{-9}$ \\
(b) & $5.3 \cdot 10^{-5}$ & $7.0 \cdot 10^{-6}$ & $4.6 \cdot 10^{-7}$ & $5.7 \cdot 10^{-8}$ & $7.0 \cdot 10^{-9}$ & $4.4 \cdot 10^{-10}$ \\
(c) & $8.3 \cdot 10^{-4}$ & $1.7 \cdot 10^{-4}$ & $1.4 \cdot 10^{-5}$ & $1.9 \cdot 10^{-6}$ & $2.4 \cdot 10^{-7}$ & $1.5 \cdot 10^{-8}$ \\
(d) & $5.5 \cdot 10^{-4}$ & $9.6 \cdot 10^{-5}$ & $7.3 \cdot 10^{-6}$ & $9.4 \cdot 10^{-7}$ & $1.2 \cdot 10^{-7}$ & $7.4 \cdot 10^{-9}$ \\
(e) & $5.7 \cdot 10^{-2}$ & $3.4 \cdot 10^{-2}$ & $1.3 \cdot 10^{-2}$ & $2.7 \cdot 10^{-3}$ & $4.5 \cdot 10^{-4}$ & $3.2 \cdot 10^{-5}$ \\
(f) & $1.3 \cdot 10^{-2}$ & $6.4 \cdot 10^{-3}$ & $7.0 \cdot 10^{-4}$ & $8.5 \cdot 10^{-5}$ & $1.4 \cdot 10^{-5}$ & $9.7 \cdot 10^{-7}$ \\
\hline
\end{tabular}

Table A.2: Error $\Delta_{r}$ of the FPT density $\rho_{j}$ from the block-by-block method for periodic input at different step sizes $h$. Input was slow (a), (b), fast (c), (d), and suprathreshold (e), (f) with weak noise for the first, strong noise for the second of each pair. The error given is relative to the numerical solution obtained with $h=2 \cdot 10^{-3}$. The FPT density was computed for $0 \leq t \leq 100$ in all cases. Parameters: (a) $\mu=0.9, q=0.1, \Omega=0.05 / \pi$, $\sigma=0.001$, (b) $\mu=0.9, q=0.1, \Omega=0.05 / \pi, \sigma=0.01$, (c) $\mu=0.9, q=0.1, \Omega=\pi, \sigma=0.05$, (d) $\mu=0.9, q=0.1, \Omega=\pi, \sigma=0.1$, (e) $\mu=1.05, q=0.5, \Omega=0.1 / \pi, \sigma=0.1$, (f) $\mu=1.05$, $q=0.5, \Omega=0.1 / \pi, \sigma=1$.

can be evaluated directly by p_train, whence this case is used as reference to validate the program.

Table A.1 gives the root mean square error

$$
\Delta_{r}=\sqrt{\sum_{j=1}^{N}\left(\rho_{j}-\rho\left(t_{j}\right)\right)^{2}}
$$

of the numerical solution $\rho_{j}$ obtained from p_train against the analytical solution $\rho(t)$ of Eq. (A.10) for a wide range of step sizes $h$. The error decreases nearly with the third power of the step size $\left(\Delta_{r} \sim h^{2.92}\right.$, correlation coefficient $r>0.999$ ), while the required CPU time grows inversely quadratic $\left(t_{\mathrm{CPU}} \sim h^{-2}\right)$. The implementation is thus validated for constant input. ${ }^{2}$

\section{Periodic input}

No first-passage time densities have been published for the Ornstein-Uhlenbeck process with time-dependent input. Thus, one may only test for the convergence of the algorithm as the step size is refined, and check for consistency with simulated spikes trains. The former is done here, while the latter is postponed to Appendix B.

The test stimulus used is $I(t)=\mu+q \cos \Omega t$. Results are given in Table A.2 for six different input conditions, comprising slow and fast, sub- and suprathreshold currents, both for weak and strong noise. Across all conditions

\footnotetext{
${ }^{2}$ Linz (1985) mentions an error scaling $\sim h^{\frac{3}{2}}$ in Chapter 10.4, which is obviously wrong, compare Table 10.3 of that book.
} 
the error scales roughly as $\Delta_{r} \sim h^{2.9}$ with correlation coefficient $r>0.999$, indicating stability. As was to be expected, the error is larger for weak noise and/or fast stimuli than for strong noise and/or slow stimuli. But even in the "worst case" (e) in Table A.2, i.e. a suprathreshold stimulus combined with weak noise, does the error decay nicely for $h<0.2$. The algorithm is thus well suited to the numerical computation of first-passage-time densities of the modulated Ornstein-Uhlenbeck process.

When choosing the stepsize, two points should be kept in mind. First of all, the FPT density will be modulated on the timescale of the input period $T$, requiring $h \ll T$. This timescale is set by the cut-off frequency for aperiodic input. Secondly, the FPT density has a sharp peak for suprathreshold input in combination with a small noise amplitude or for very strong noise. The stepsize may have to be very small to resolve this peak properly and to avoid numerical instabilities. 


\section{Appendix B}

\section{Spike Train Simulation}

Neuronal spike trains can be simulated efficiently using a method proposed by Gillespie (1996). It exploits the fact that the moment equations for the unbounded Ornstein-Uhlenbeck process can be solved analytically. This permits the use of - in principle - arbitrarily large time steps. In the presence of an absorbing boundary, an adaptive stepsize algorithm must be used.

\section{B.1 Algorithm}

A train of spikes at times $t_{0}, t_{1}, \ldots, t_{j}$ is generated by simulating the trajectory of the membrane potential $v(t)$ as defined by Eq. (2.20) from the reset potential $v_{R}$ to the threshold $\Theta=1$. Upon threshold crossing, the current time is recorded as time of the spike, the potential reset, and the next interval simulated. The algorithm for the simulation of the subthreshold membrane potential is derived and tested here.

The evolution of the subthreshold membrane potential in between two spikes is simulated by numerical integration of the Langevin Eq. (2.20a)

$$
\dot{v}(t)=-v(t)+g(t)+\sigma \xi(t), \quad\langle\xi(t)\rangle=0, \quad\left\langle\xi(t) \xi\left(t^{\prime}\right)\right\rangle=\delta\left(t-t^{\prime}\right),
$$

from $v=v_{R}$ to $v=\Theta=1$.

In the absence of an absorbing threshold $(\Theta=\infty)$, the Langevin equation can be integrated formally, yielding for arbitrary step size $h>0$

$$
v(t+h)=\left[v(t)+\int_{0}^{h} g(t+s) \mathrm{e}^{s} \mathrm{~d} s\right] e^{-h}+\left[\int_{0}^{h} \xi(t+s) \mathrm{e}^{s} \mathrm{~d} s\right] \mathrm{e}^{-h} .
$$

The white noise $\xi(t)$ is correlated neither to the potential $v(t)$ nor the input $g(t)$, so that mean and variance of $v(t+h)$ become

$$
\begin{aligned}
\langle v(t+h)\rangle & =[v(t)+\hat{G}(t, h)] \mathrm{e}^{-h}, \\
\Sigma^{2}(h) & =\left\langle v(t+h)^{2}\right\rangle-\langle v(t+h)\rangle^{2}=\frac{\sigma^{2}}{2}\left(1-\mathrm{e}^{-2 h}\right),
\end{aligned}
$$


with $\hat{G}(t, h)=\int_{0}^{h} g(t+s) \mathrm{e}^{s} \mathrm{~d} s$. Since the potential $v(t+h)$ depends linearly on the Gaussian process $\xi(t)$, the mean and variance completely describe the distribution of $v(t+h)$.

Thus, starting from $v_{0}=v_{R}$ at $t_{0}=\hat{t}$, the iteration rule

$$
\begin{aligned}
v_{n+1} & =\left\langle v\left(t_{n}+h_{n}\right)\right\rangle+\operatorname{nrm}\left(0, \Sigma_{n}\right)=\left[v_{n}+\hat{G}\left(t_{n}, h_{n}\right)\right] \mathrm{e}^{-h_{n}}+\operatorname{nrm}\left(0, \Sigma_{n}\right), \\
t_{n+1} & =t_{n}+h_{n} \\
\Sigma_{n} & =\Sigma\left(h_{n}\right)=\sigma \sqrt{\frac{1-\mathrm{e}^{-2 h_{n}}}{2}}
\end{aligned}
$$

will generate a realization of the unbounded Ornstein-Uhlenbeck process. Here, $\operatorname{nrm}\left(0, \Sigma_{n}\right)$ is a normally distributed random number with zero mean and variance $\Sigma_{n}$ (Knuth 1998). The stepsize $h_{n}>0$ may vary from step to step.

The argument given above does no longer hold precisely in the presence of an absorbing threshold at $\Theta<\infty$. But as long as the membrane potential is sufficiently far below threshold to make a threshold crossing within the next time step practically impossible, the iteration rule of Eq. (B.3) will introduce only negligible error. For subthreshold input, ${ }^{1}$ threshold crossings can only be induced by the random term in the iteration rule. Thus, if the stepsize $h_{n}$ is chosen such that

$$
\Sigma_{n}=\Sigma\left(h_{n}\right) \leq \frac{\Theta-v_{n}}{\vartheta},
$$

the probability of a threshold crossing in the next step will be less than (1erf $\vartheta) / 2$. To avoid an infinite slowing down of the simulation as the threshold is approached, a lower bound $h_{\min }$ of the stepsize is required. The upper bound $h_{\max }$ is given by the fastest timescale of the input $g(t)$. This yields the following rule for the stepsize

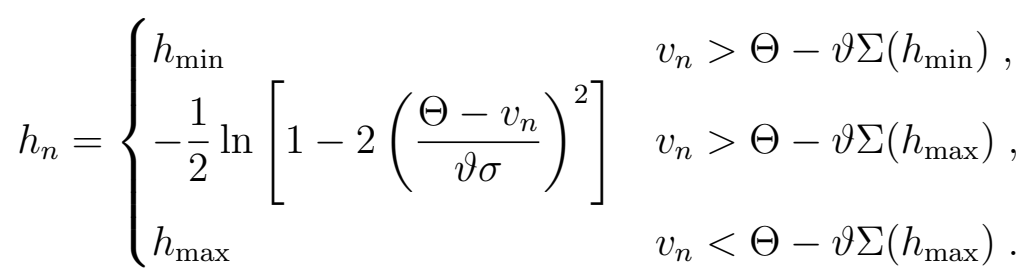

The parameters $\vartheta$ and $h_{\text {min }}$ determine the degree to which the validity of the approximation is enforced. For $\vartheta=5$, which has been used for all simulations presented here, the threshold-crossing probability is $<10^{-6}$, i.e. the influence of the threshold is irrelevant as long as $v_{n} \leq \Theta-\vartheta \Sigma\left(h_{\min }\right)$. The choice of the lower bound $h_{\text {min }}$ is discussed below. The upper bound has typically been

\footnotetext{
${ }^{1}$ For very strong suprathreshold input $(g(t) \gg \Theta)$, the upper bound $h_{\text {max }}$ may have to be reduced to assure that $v_{n}+\hat{G}(t, h)<\Theta$. Such cases have not been studied here.
} 


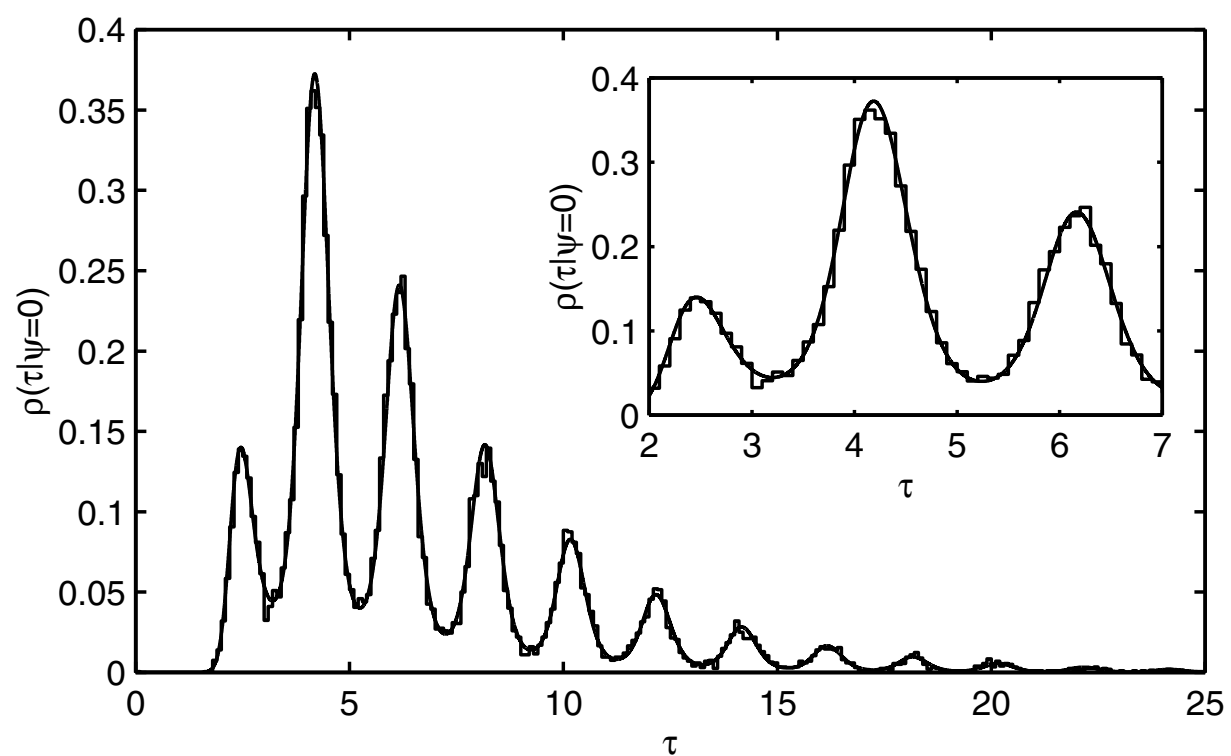

Figure B.1: Distribution of simulated interspike intervals (boxed line) and predicted density $\rho(t \mid \psi=0)$ (smooth line) for case (d) of Table B.1; the first three modes are shown in detail in the inset. The histogram is based on 20000 spikes, and the bin width is $\delta \tau=0.1$.

set to $h_{\max }=0.01$, while the fastest stimuli studied had periods of $T=0.4$. It is essentially this bound that determines the computer time required for simulations.

It is important to note that the stepsize has to be chosen before a step is executed, and that a step that has been executed must not be rejected after a threshold crossing was detected: This would introduce correlations in the random numbers drawn in the iteration process.

The simulation algorithm is implemented as program p_train for periodic input and as program ap_train for aperiodic input as defined in Chapter 2.3. Both programs are available in electronic form (Plesser 1999).

\section{B.2 Validation}

The simulation algorithm is tested for the same periodic stimuli employed in the validation of the block-by-block method in Appendix A.2. For each parameter set, 20000 interspike intervals were simulated and compared to true ISI density $\rho(\tau \mid 0)$ given by Eq. (2.33). The example shown in Fig. B.1 indicates excellent agreement.

A quantitative test of the goodness-of-fit is provided by the KolmogorovSmirnov statistic $D_{n}$ (Stuart, Ord, and Arnold 1999). Let $\tau_{(1)}<\tau_{(2)}<\ldots<$ $\tau_{(n)}$ be the simulated intervals from p_train ordered by rank, with empirical 


\begin{tabular}{llllll}
\hline$h_{\min }$ & $1.0 \cdot 10^{-2}$ & $1.0 \cdot 10^{-3}$ & $1.0 \cdot 10^{-4}$ & $1.0 \cdot 10^{-8}$ & $1.0 \cdot 10^{-10}$ \\
\hline (a) & $6.4 \cdot 10^{-2}$ & $1.6 \cdot 10^{-2}$ & $1.1 \cdot 10^{-2}$ & $9.8 \cdot 10^{-3}$ & $4.5 \cdot 10^{-3}$ \\
(b) & $4.3 \cdot 10^{-2}$ & $1.7 \cdot 10^{-2}$ & $4.8 \cdot 10^{-3}$ & $6.0 \cdot 10^{-3}$ & $4.2 \cdot 10^{-3}$ \\
(c) & $5.9 \cdot 10^{-2}$ & $2.2 \cdot 10^{-2}$ & $8.5 \cdot 10^{-3}$ & $4.6 \cdot 10^{-3}$ & $8.8 \cdot 10^{-3}$ \\
(d) & $4.5 \cdot 10^{-2}$ & $2.2 \cdot 10^{-2}$ & $1.5 \cdot 10^{-2}$ & $6.1 \cdot 10^{-3}$ & $4.9 \cdot 10^{-3}$ \\
(e) & $6.8 \cdot 10^{-2}$ & $1.6 \cdot 10^{-2}$ & $6.8 \cdot 10^{-3}$ & $5.0 \cdot 10^{-3}$ & $5.4 \cdot 10^{-3}$ \\
(f) & $5.4 \cdot 10^{-2}$ & $2.2 \cdot 10^{-2}$ & $8.1 \cdot 10^{-3}$ & $1.0 \cdot 10^{-2}$ & $6.7 \cdot 10^{-3}$ \\
\hline
\end{tabular}

Table B.1: Kolmogorov-Smirnov statistic $D_{n}$ for 20000 interspike intervals generated by p_train at different minimal step sizes $h_{\min }$. The hypothesis that intervals are distributed according to $\rho(\tau \mid 0)$ is accepted at the $1 \%$ level if $D_{n}<0.0115$. Input was slow (a), (b), fast (c), (d), and suprathreshold (e), (f) with weak noise for the first, strong noise for the second of each pair. For precise parameters, see Table A.2.

distribution function

$$
S_{n}(x)= \begin{cases}0 & x<\tau_{(1)} \\ \frac{r}{n} & \tau_{(r)} \leq x<\tau_{(r+1)} \\ 1 & \tau_{(n)}<x\end{cases}
$$

and let $P(t \mid \psi=0)=\int_{0}^{t} \rho(\tau \mid \psi=0) \mathrm{d} \tau$ be the distribution function obtained from $p_{-}$fptd. The Kolmogorov-Smirnov statistic is then defined as

$$
D_{n}=\sup _{t \geq 0}\left|S_{n}(t)-P(t \mid \psi=0)\right| \text {. }
$$

The hypothesis that the $n$ simulated intervals $\tau_{j}$ are distributed according to $\rho(t \mid \psi=0)$ is rejected at the $1 \%$ level if $\sqrt{n} D_{n}>1.63$ (Bronstein and Semendjajew 1989). For the given sample size of $n=20000$, this corresponds to $D_{n}>0.0115$. The true distribution $\rho(\tau \mid \psi=0)$ is computed with p_fptd at very high resolution.

Results are given in Table B.1. For all six sample cases, the simulated spike trains and the distribution $\rho(\tau \mid 0)$ are at disagreement for $h_{\min }>10^{-4}$ at the $1 \%$ level. For $h_{\min } \leq 10^{-8}$ the Kolmogorov-Smirnov test indicates agreement between simulation and theory even for fast and suprathreshold input. Therefore, $h_{\min }=10^{-9}$ has been employed in the simulations reported here.

As the block-by-block method and the simulation algorithm yield the same results independent of each other, these methods cross-validate each other and can be considered reliable. 


\section{References}

Abeles, M. (1982). Role of the cortical neuron: integrator or coincidence detector. Israel J Med Sci 18, 83-92.

Abeles, M. (1991). Corticonics. Cambridge, UK: Cambridge University Press.

Aertsen, A., G. L. Gerstein, M. K. Habib, and G. Palm (1989). Dynamics of neuronal firing correlation: Modulation of "effective connectivity". $J$ Neurophysiol 61, 900-917.

Anderson, D. J., J. E. Rose, J. E. Hind, and J. F. Brugge (1971). Temporal position of discharges in single auditory nerve fibers within the cycle of a sine-wave stimulus: Frequency and intensity effects. J Acoust Soc Am 49, 1131-1139.

Bair, W. and C. Koch (1996). Temporal precision of spike trains in extrastriate cortex of the behaving macaque monkey. Neural Comput 8, 1185-1202.

Bair, W., C. Koch, W. Newsome, and K. Britten (1994). Power spectrum analysis of bursting cells in area MT in the behaving monkey. $J \mathrm{Neu}$ rosci 14, 2870-2892.

Baker, C. T. H. (1977). The Numerical Treatment of Integral Equations. Oxford: Clarendon Press.

Benzi, R., A. Sutera, and A. Vulpiani (1981). The mechanism of stochastic resonance. J Phys A 14, L453-L457.

Berdichevsky, V. and M. Gitterman (1996). Stochastic resonance in a bistable piecewise potential: analytical solution. J Phys A 29, L447L452.

Bezrukov, S. M. and I. Vodyanoy (1997). Signal transduction across alamethicin ion channels in the presence of noise. Biophys $J$ 73, 2456-2464.

Boven, K.-H. and A. Aertsen (1990). Dynamics of activity in neuronal networks give rise to fast modulations of functional connectivity. In R. Eckmiller, G. Hartmann, and G. Hauske (Eds.), Parallel Processing in Neural Systems and Computers, pp. 53-56. North-Holland. 
Braun, H. A., K. Schäfer, and H. Wissing (1984). Theorien und Modelle zum Übertragungsverhalten thermosensitiver Rezeptoren. Funkt Biol Med 3, 26-36.

Bronstein, I. N. and K. A. Semendjajew (1989). Taschenbuch der Mathematik. Thun: Harri Deutsch.

Bryant, H. L. and J. P. Segundo (1976). Spike inititation by transmembrane current: a white-noise analysis. J Physiol 260, 279-314.

Bugmann, G., C. Christodoulou, and J. G. Taylor (1997). Role of temporal integration and fluctuation detection in the highly irregular firing of a leaky integrator neuron model with partial reset. Neural Comput 9, 9851000.

Bulsara, A., E. W. Jacobs, T. Zhou, F. Moss, and L. Kiss (1991). Stochastic resonance in a single neuron model: Theory and analog simulation. $J$ Theor Biol 152, 531-555.

Bulsara, A. R., T. C. Elston, C. R. Doering, S. B. Lowen, and K. Lindenberg (1996). Cooperative behavior in periodically driven noisy integrate-fire models of neuronal dynamics. Phys Rev E 53, 3958-3969.

Bulsara, A. R., S. B. Lowen, and C. D. Rees (1994). Cooperative behavior in the periodically modulated Wiener process: Noise-induced complexity in a model neutron [sic]. Phys Rev E 49, 4989-5000.

Buonocore, A., A. G. Nobile, and L. M. Ricciardi (1987). A new integral equation for the evaluation of first-passage-time probability densities. Adv Appl Prob 19, 784-800.

Burkitt, A. N. and G. M. Clark (1999a). Analysis of integrate-and-fire neurons: Synchronization of synaptic input and spike output. Neural Comput 11, 871-901.

Burkitt, A. N. and G. M. Clark (1999b). Synchronization of the neural response to noisy periodic input. Submitted.

Capocelli, R. M. and L. M. Ricciardi (1971). Diffusion approximation and first passage time problem for a model neuron. Kybernetik 8, 214-223.

Chialvo, D. R., A. Longtin, and J. Müller-Gerking (1997). Stochastic resonance in models of neuronal ensembles. Phys Rev E 55, 1798-1808.

Choi, M. H., R. F. Fox, and P. Jung (1998). Quantifying stochastic resonance in bistable systems: Response vs residence-time distribution functions. Phys Rev E 57, 6335-6344.

Collins, J. J., C. C. Chow, A. C. Capela, and T. T. Imhoff (1996). Aperiodic stochastic resonance. Phys Rev E 54, 5575-5584.

Collins, J. J., C. C. Chow, and T. T. Imhoff (1995). Aperiodic stochastic resonance in excitable systems. Phys Rev E 52, R3321-R3324. 
Collins, J. J., T. T. Imhoff, and P. Grigg (1996). Noise-enhanced information transmission in rat SA1 cutaneous mechnoreceptors via aperiodic stochastic resonance. J Neurophysiol 76, 642-645.

Cordo, P., J. T. Inglis, S. Verschueren, J. J. Collins, D. M. Merfeld, S. Rosenblum, S. Buckley, and F. Moss (1996). Noise in human muscle spindels. Nature 383, 769-770.

Cover, T. M. and J. A. Thomas (1991). Elements of Information Theory. New York: Wiley.

Cox, D. R. and P. A. W. Lewis (1966). The Statistical Analysis of Series of Events. London: Methuen.

Cox, D. R. and H. D. Miller (1965). The Theory of Stochastic Processes. London: Methuen\&Co.

Daley, D. J. and D. Vere-Jones (1988). An Introduction to the Theory of Point Processes. New York: Springer.

deCharms, R. C. and M. M. Merzenich (1996). Primary cortical representation of sounds by the coordination of action-potential timing. Nature 381 , 610-613.

DeWeese, M. and W. Bialek (1995). Information flow in sensory neurons. Nuovo Cimento D 17, 733-741.

Diesmann, M., M.-O. Gewaltig, and A. Aertsen (1999). Conditions for stable propagation of synchronous spiking in cortical neural networks. Nature. In the press.

Douglass, J. K., L. Wilkens, E. Pantazelou, and F. Moss (1993). Noise enhancement of information transfer in crayfish mechanoreceptors by stochastic resonance. Nature 365, 337-340.

Ermentrout, B. (1996). Type I membranes, phase resetting curves, and synchrony. Neural Comput 8, 979-1001.

Eyink, G. L. (1998). Linear stochastic models of nonlinear dynamical systems. Phys Rev E 58, 6975-6991.

Fauve, S. and F. Heslot (1983). Stochastic resonance in a bistable system. Phys Lett A 97, 5-7.

Feller, W. (1970). An Introduction to Probability Theory and Its Applications (Third ed.), Volume 1. New York: John Wiley \& Sons.

Feller, W. (1971). An Introduction to Probability Theory and Its Applications (Second ed.), Volume 2. New York: John Wiley \& Sons.

Feng, J. (1997). Behaviors of spike output jitter in the integrate-and-fire model. Phys Rev Lett 79, 4505-4508. 
Franklin, J. and W. Bair (1995). The effect of a refractory period on the power spectrum of neuronal discharge. SIAM J Appl Math 55, 10741093.

Gabbiani, F., W. Metzner, R. Wessel, and C. Koch (1996). From stimulus encoding to feature extraction in weakly electric fish. Nature 384, 564567.

Galdi, V., V. Pierro, and I. M. Pinto (1998). Evaluation of stochasticresonance-based detectors of weak harmonic signals in additive white Gaussian noise. Phys Rev E 57, 6470-6479.

Gammaitoni, L. (1995). Stochastic resonance and the dithering effect in threshold physical systems. Phys Rev E 52, 4691-4698.

Gammaitoni, L., P. Hänggi, P. Jung, and F. Marchesoni (1998). Stochastic resonance. Rev Mod Phys 70, 223-287.

Gammaitoni, L., F. Marchesoni, E. Menichella-Saetta, and S. Santucci (1989). Stochastic resonance in bistable systems. Phys Rev Lett 62, 349352.

Gammaitoni, L., F. Marchesoni, and S. Santucci (1995). Stochastic resonance as a bona fide resonance. Phys Rev Lett 74, 1052-1055.

Geisler, C. D. (1968). A model of the peripheral auditory system responding to low-frequency tones. Biophys J 8, 1-15.

Gerstein, G. L. and B. Mandelbrot (1964). Random walk models for the spike activity of a single neuron. Biophys $J$ 4, 41-68.

Gerstner, W. (1995). Time structure of the activity in neural network models. Phys Rev E 51, 738-758.

Gerstner, W. (1999a). Populations of spiking neurons. See Maass and Bishop (1999), Chapter 10, pp. 261-295.

Gerstner, W. (1999b). Spiking neurons. See Maass and Bishop (1999), Chapter 1 , pp. 3-54.

Gerstner, W., R. Kemptner, J. L. van Hemmen, and H. Wagner (1996). A neuronal learning rule for sub-millisecond temporal coding. Nature 383, 76-78.

Gerstner, W. and J. L. van Hemmen (1992). Associative memory in a network of 'spiking' neurons. Network 3, 139-164.

Giacomelli, G., F. Marin, and I. Rabbiosi (1999). Stochastic and bona fide resonance: An experimental investigation. Phys Rev Lett 82, 675-678.

Gihman, I. I. and A. V. Skorohod (1972). Stochastic Differential Equations. Berlin: Springer. 
Gillespie, D. T. (1996). Exact numerical simulation of the OrnsteinUhlenbeck process and its integral. Phys Rev E 54, 2084-2091.

Gingl, Z., L. B. Kiss, and F. Moss (1995). Non-dynamical stochastic resonance: Theory and experiments with white and arbitrarily coloured noise. Europhys Lett 29, 191-196.

Gitterman, M. and G. H. Weiss (1995). Coherent stochastic resonance in the presence of a field. Phys Rev E 52, 5708-5713.

Glantz, R. (1999). Private communication.

Gluckman, B. J., T. I. Netoff, E. J. Neel, W. L. Ditto, M. L. Spano, and S. J. Schiff (1996). Stochastic resonance in a neuronal network from mammalian brain. Phys Rev Lett 77, 4098-4101.

Gluss, B. (1967). A model for neuron firing with exponential decay of potential resulting in diffusion equations for probability density. Bull Math Biophysics 29, 233-243.

Goldberg, J. M. and P. B. Brown (1969). Response of binaural neurons of dog superior olivary complex to dichotic tonal stimuli: Some physiological mechanisms of sound localization. J Neurophysiol 32, 613-636.

Golomb, D., J. Hertz, S. Panzeri, A. Treves, and B. Richmond (1997). How well can we estimate the information carried in neuronal responses from limited samples? Neural Comput 9, 649-665.

Gradsteyn, I. S. and I. M. Ryzhik (1980). Table of Integrals, Series and Products. New York: Academic Press.

Herrmann, A. and W. Gerstner (1999). Effect of noise on neuron transient response. In J. Bower (Ed.), CNS 1999 Abstracts, Pasadena, CA, p. 86. Caltech.

Herrmann, M., J. A. Hertz, and A. Prügel-Bennett (1995). Analysis of synfire chains. Network 6, 403-414.

Holt, G. R. and C. Koch (1999). Electrical interactions via the extracellular potentials near cell bodies. J Comp Neurosci 6, 169-184.

Hopfield, J. J. (1995). Pattern recognition computation using action potential timing for stimulus representation. Nature 376, 33-36.

Inoue, J., S. Sato, and L. M. Ricciardi (1997). A note on the moments of the first-passage time of the Ornstein-Uhlenbeck process with a reflecting boundary. Ricerche di Matematica 46, 87-99.

Jaramillo, F. and K. Wiesenfeld (1998). Mechanoelectrical transduction assisted by Brownian motion: a role for noise in the auditory system. Nature Neurosci 1, 384-388. 
Johannesma, P. I. M. (1968). Diffusion models of the stochastic activity of neurons. In E. R. Caianiello (Ed.), Neural Networks, pp. 116-144. Berlin: Springer.

Johnston, D. and S. M.-S. Wu (1995). Foundations of Cellular Neurophysiology. Cambridge, MA: MIT Press.

Jung, P. (1993). Periodically driven stochastic systems. Phys Rep 234, 175295.

Jung, P. (1994). Threshold devices: Fractal noise and neural talk. Phys Rev E 50, 2513-2522.

Kallianpur, G. (1983). On the diffusion approximation to a discontinuous model for a single neuron. In P. K. Sen (Ed.), Contributions to Statistics, pp. 247-258. Amsterdam: North-Holland.

Kanamaru, T., T. Horita, and Y. Okabe (1999). Stochastic resonance for the superimposed periodic pulse train. Phys Lett A 255, 23-30.

Kandel, E. R., J. H. Schwartz, and T. M. Jessell (1991). Principles of Neural Science (Third ed.). East Norwalk, CT: Appleton\&Lange.

Keener, J. P., F. C. Hoppensteadt, and J. Rinzel (1981). Integrate-and-fire models of nerve membrane response to oscillatory input. SIAM J Appl Math 41, 503-517.

Kempter, R., W. Gerstner, J. L. van Hemmen, and H. Wagner (1998). Extracting out oscillations: neural coincidence detection with periodic spike input. Neural Comput 10, 1987-2017.

Kiang, N. Y.-S. (1965). Discharge Patterns of Single Fibers in the Cat's Auditory Nerve. Cambridge, MA: MIT Press.

Knudsen, E. I. (1984). Synthesis of a neural map of auditory space in the owl. In G. M. Edelman, W. E. Gall, and W. M. Cowan (Eds.), Dynamic Aspects of Neocortical Function, Chapter 11, pp. 375-396. Neuroscience Research Foundation.

Knuth, D. E. (1998). The Art of Computer Programming (Third ed.), Volume 2. Reading, MA: Addison-Wesley.

Konishi, M. (1991). Deciphering the brain's codes. Neural Comput 3, 1-18.

Lánský, P. (1984). On approximations of Stein's neuronal model. J Theor Biol 10\%, 631-647.

Lánský, P. (1997). Sources of periodical force in noisy integrate-and-fire models of neuronal dynamics. Phys Rev E 55, 2040-2043.

Lánský, P. and V. Lánská (1987). Diffusion approximation of the neuronal model with synaptic reversal potentials. Biol Cybern 56, 19-26. 
Lánský, P. and J. P. Rospars (1995). Ornstein-Uhlenbeck model neuron revisited. Biol Cybern 72, 397-406.

Lapicque, L. (1907). Recherches quantitatives sur l'excitation électrique des nerfs traitée comme une polarization. J Physiol Pathol Gen 9, 620-635.

Lasota, A. and M. C. Mackey (1994). Chaos, Fractals, and Noise: Stochastic Aspects of Dynamics (Second ed.). New York: Springer.

Laughlin, S. B., R. R. de Ruyter van Steveninck, and J. C. Anderson (1998). The metabolic cost of neural information. Nature Neurosci 1, 36-41.

Levin, J. E. and J. P. Miller (1996). Broadband neural encoding in the cricket cercal sensory system enhanced by stochastic resonance. Nature 380, $165-168$.

Linz, P. (1985). Analytical and Numerical Methods for Volterra Equations. Philadelphia: SIAM.

Liu, F., J. F. Wang, and W. Wang (1999). Frequency sensitivity in weak signal detection. Phys Rev E 59, 3453-3460.

Longtin, A. (1993). Stochastic resonance in neuron models. J Stat Phys 70, 309-327.

Longtin, A., A. Bulsara, and F. Moss (1991). Time-interval sequences in bistable systems and the noise-induced transmission of information by sensory neurons. Phys Rev Lett 67, 656-659.

Longtin, A. and K. Hinzer (1996). Encoding with bursting, subthreshold oscillations, and noise in mammalian cold receptors. Neural Comput 8, $215-255$.

Lukes, T. (1961). The statistical properties of sequences of stochastic pulses. Proc Phys Soc Lond 78, 153-168.

Maass, W. and C. M. Bishop (Eds.) (1999). Pulsed Neural Networks. Cambridge, MA: MIT Press.

Maass, W. and T. Natschläger (1999). A model for fast analog computation based on unreliable synapses. Neural Comput. In the press.

Mainen, Z. F. and T. J. Sejnowski (1995). Reliability of spike timing in neocortical neurons. Science 268, 1503-1506.

Maršálek, P., C. Koch, and J. Maunsell (1997). On the relationship between synaptic input and spike output jitter in individual neurons. Proc Natl Acad Sci USA 94, 735-740.

Matsuyama, Y., K. Shirai, and K. Akizuki (1974). On some properties of stochastic information processes in neurons and neuron populations. $K y$ bernetik 15, 127-145. 
McCulloch, W. S. and W. Pitts (1943). A logical calculus of the ideas immanent in nervous activity. Bull Math Biophysics 5, 115-133.

McNamara, B. and K. Wiesenfeld (1989). Theory of stochastic resonance. Phys Rev A 39, 4854-4869.

McNamara, B., K. Wiesenfeld, and R. Roy (1988). Observation of stochastic resonance in a ring laser. Phys Rev Lett 60, 2626-2629.

Morse, R. P. and E. F. Evans (1996). Enhancement of vowel coding for cochlear implants by addition of noise. Nature Med 2, 928-932.

Neiman, A., X. Pei, D. Russell, W. Wojtenek, L. Wilkens, F. Moss, H. A. Braun, M. T. Huber, and K. Voigt (1999). Synchronization of the noisy electrosensitive cells in the paddlefish. Phys Rev Lett 82, 660-663.

Nishimura, H., K. Hashikawa, K. Doi, T. Iwaki, Y. Watanabe, H. Kusuoka, T. Nishimura, and T. Kubo (1999). Sign language 'heard' in the auditory cortex. Nature 39\%, 116.

Perkel, D. H., G. L. Gerstein, and G. P. Moore (1967). Neuronal spike trains and stochastic point processes. Biophys $J$ \%, 391-418.

Plesser, H. E. (1999). Numerical methods for the modulated OrnsteinUhlenbeck process. Technical report, MPI für Strömungsforschung, Göttingen. In preparation.

Plesser, H. E. and T. Geisel (1999). Markov analysis of stochastic resonance in a periodically driven integrate-and-fire neuron. Phys Rev E 59, 70087017 .

Plesser, H. E. and W. Gerstner (1999). Neural Comput. In the press.

Plesser, H. E. and S. Tanaka (1997). Stochastic resonance in a model neuron with reset. Phys Lett A 225, 228-234.

Press, W. H., S. A. Teukolsky, W. T. Vetterling, and B. P. Flannery (1992). Numerical Recipes in $C$ (Second ed.). Cambridge, UK: Cambridge University Press.

Priestley, M. B. (1996). Spectral Analysis and Time Series. London: Academic Press.

Rieke, F., D. Warland, R. de Ruyter van Steveninck, and W. Bialek (1997). Spikes: Exploring the Neural Code. Cambridge, MA: MIT Press.

Rose, J. E., J. F. Brugge, D. J. Anderson, and J. E. Hind (1967). Phaselocked response to low-frequency tones in single auditory nerve fibers of the squirrel monkey. J Neurophysiol 30, 769-793.

Rotter, S. (1994). Wechselwirkende stochastische Punktprozesse. Thun: Harri Deutsch. 
Russell, D. F. and F. Moss (1998). Animal behavior enhanced by noise. In J. Bower (Ed.), CNS 1998 Abstracts, Pasadena, CA, p. 137. Caltech.

Sarpeshkar, R. (1998). Analog versus digital: Extrapolation from electronics to neurobiology. Neural Comput 10, 1601-1638.

Scharstein, H. (1979). Input-output relationship of the leaky-integrator neuron model. J Math Biol 8, 403-420.

Schrödinger, E. (1915). Zur Theorie der Fall- und Steigversuche an Teilchen mit Brownscher Bewegung. Physikalische Zeitschrift 16, 289-295.

Scott, D. W. (1992). Multivariate Density Estimation. New York: Wiley.

Segev, I. and R. E. Burke (1998). Compartmental models of complex neurons. In C. Koch and I. Segev (Eds.), Methods in Neural Modeling (Second ed.)., Chapter 3, pp. 93-136. Cambridge, MA: MIT Press.

Shadlen, M. and W. T. Newsome (1998). The variable discharge of cortical neurons: Implications for connectivity, computation and information coding. J Neurosci 18, 3870-3896.

Shimokawa, T., K. Pakdaman, and S. Sato (1999a). Time-scale matching in the response of a leaky integrate-and-fire neuron model to periodic stimulus with additive noise. Phys Rev E 59, 3427-3443.

Shimokawa, T., A. Rogel, K. Pakdaman, and S. Sato (1999b). Stochastic resonance and spike-timing precision in an ensemble of leaky integrate and fire neurons. Phys Rev E 59, 3461-3470.

Siegert, A. J. F. (1951). On the first passage time probability problem. Phys Rev 81, 617-623.

Simonotto, E., M. Riani, C. Seife, M. Roberts, J. Twitty, and F. Moss (1997). Visual perception of stochastic resonance. Phys Rev Lett 78, 1186-1189.

Spiridon, M. and W. Gerstner (1999). Noise spectrum and signal transmission through a population of spiking neurons. Network 10, 257-272.

Srebro, R. and P. Malladi (1999). Stochastic resonance of the visually evoked potential. Phys Rev E 59, 2566-2570.

Stein, R. B. (1965). A theoretical analysis of neuronal variability. Biophys $J$ 5, 173-194.

Stemmler, M. (1996). A single spike suffices: the simplest form of stochastic resonance in neuron models. Network 7, 687-716.

Stevens, C. F. and A. M. Zador (1998). Input synchrony and the irregular firing of cortical neurons. Nature Neurosci 1, 210-217.

Strong, S. P., R. Koberle, R. R. de Ruyter van Steveninck, and W. Bialek (1998). Entropy and information in neural spike trains. Phys Rev Lett 80, 197-200. 
Stuart, A., J. K. Ord, and S. Arnold (1999). Classical Inference and the Linear Model (Sixth ed.), Volume 2A of Kendall's Advanced Theory of Statistics. London: Arnold.

Sugiyama, H., G. P. Moore, and D. H. Perkel (1970). Solutions for a stochastic model of neuronal spike production. Math Biosci 8, 323-341.

Tateno, T. (1998). Characterization of stochastic bifurcations in a simple biological oscillator. J Statist Phys 92, 675-705.

Tateno, T., S. Doi, S. Sato, and L. M. Ricciardi (1995). Stochastic phase lockings in a relaxation oscillator forced by a periodic input with additive noise: A first-passage time approach. J Statist Phys 78, 917-935.

The MathWorks, Inc. (1998). MATLAB Function Reference. Natick, MA, USA: The MathWorks, Inc.

The MathWorks, Inc. (1999). Optimization Toolbox User's Guide. Natick, MA, USA: The MathWorks, Inc.

Tougaard, J. (1999). Stochastic resonance and the role of noise in hearing. In N. Elsner and U. Eysel (Eds.), Göttingen Neurobiology Report 1999, Volume II, Stuttgart, p. 261. Thieme.

Troyer, T. W. and K. D. Miller (1997). Physiological gain leads to high ISI variability in a simple model of a cortical regular spiking cell. Neural Comput 9, 971-983.

Tuckwell, H. C. (1988). Introduction to Theoretical Neurobiology, Volume 1. Cambridge, UK: Cambridge University Press.

Tuckwell, H. C. (1989). Stochastic Processes in the Neurosciences. Philadelphia: SIAM.

Tuckwell, H. C. and D. K. Cope (1980). Accuracy of neuronal interspike times calculated from a diffusion approximation. J Theor Biol 83, 377387.

Uhlenbeck, G. E. and L. S. Ornstein (1930). On the theory of the Brownian motion. Phys Rev 36, 823-841.

van Kampen, N. G. (1985). Elimination of fast variables. Phys Rep 124, 69-160.

van Kampen, N. G. (1992). Stochastic Processes in Physics and Chemistry (Second ed.). Amsterdam: North-Holland.

van Vreeswijk, C. and H. Sompolinsky (1996). Chaos in neuronal networks with balanced excitatory and inhibitory activity. Science 274, 1724-1726.

von Mises, R. (1964). Mathematical Theory of Probability and Statistics. New York: Academic Press. Ed. by H. Geiringer. 
Wiesenfeld, K. and F. Jaramillo (1998). Minireview of stochastic resonance. Chaos 8, 539-548.

Wiesenfeld, K., D. Pierson, E. Pantazelou, C. Dames, and F. Moss (1994). Stochastic resonance on a circle. Phys Rev Lett 72, 2125-2129.

Wilkens, L. A., D. F. Russell, X. Pei, and C. Gurgens (1997). The paddlefish rostrum functions as an electrosensory antenna in plankton feeding. Proc $R$ Soc Lond B 264, 1723-1729.

Wilson, H. R. and J. D. Cowan (1972). Excitatory and inhibitory interactions in localized populations of model neurons. Biophys J 12, 1-24.

Zador, A. (1998). Impact of synaptic unreliability on the information transmitted by spiking neurons. J Neurophysiol 79, 1219-1229. 



\section{Publications}

H. E. Plesser and S. Tanaka

Stochastic resonance in a model neuron with reset.

Physics Letters A 225, 228-234, 1997.

H. E. Plesser and S. Tanaka

Stochastic resonance in a model neuron.

In N. Elsner and H. Wässle (Eds.), From Membrane to Mind, Proceedings of the 25th Göttingen Neurobiology Conference, Volume II, p. 1010. Stuttgart: Thieme, 1997.

H. E. Plesser

Noise turns integrate-fire neuron into bandpass filter.

In N. Elsner and R. Wehner (Eds.), New Neuroethology on the Move, Proceedings of the 26th Göttingen Neurobiology Conference, Volume II, p. 760. Stuttgart: Thieme, 1998.

H. E. Plesser and T. Geisel

Markov analysis of stochastic resonance in a periodically driven integrate-and-fire neuron.

Physical Review E 59, 7008-7017, 1999.

H. E. Plesser and T. Geisel

Bandpass properties of integrate-fire neurons.

Neurocomputing 26-27, 229-235, 1999.

H. E. Plesser and W. Gerstner

Noise in integrate-and-fire neurons: from stochastic input to escape rates.

To appear in Neural Computation, 1999.

H. E. Plesser and W. Gerstner

Escape noise models for noisy integrate-and-fire neurons.

To appear in Neurocomputing, 2000. 



\section{Acknowledgments}

In the course of the past four years, many people have contributed to my work in different places around the globe. I would like to mention them in chronological order.

Angela Hilgers, Claudia Berghaus, and Uwe Kahlert first introduced me to the concept of stochastic resonance while I was working on my diploma thesis under the auspices of Jürgen Schnakenberg in Aachen.

Shigeru Tanaka as head of the Neural Modeling Laboratory of RIKEN's Frontier Research Program in Wako near Tokyo took the risk of accepting me as a postgraduate student who hardly knew what a receptive field was. He gave me a good start into the neurosciences. Ko Sakai, Tomoki Fukai, Hideyuki Cateau, Siegfried Bös, and Michael Herrmann were helpful and inspiring colleagues. Howard Hua Yang first mentioned the term point process to me, while Mitsuyuki Katakame suggested that I should "warm up" on the integrate-and-fire neuron before taking to the FitzHugh-Nagumo model. Most likely he did not intend to keep me busy for the better part of three years. Xiao Yan Su and Ko Sakai share the praise for tending to the computer zoo. I am indebted to RIKEN and Studienstiftung for a generous stipend that made life in the world's most expensive city affordable.

Bernhard Gaese and Hermann Wagner at the Institut für Biologie II, RWTH Aachen, did their best to train me as an experimental neurophysiologist. I cherished the warm atmosphere of the Wagner group and the inside view of "real neuroscience" I was allowed to gain there. I beg their pardon for leaving just as they had taught me how to get an electrode into a rat's brain, but I found myself to be a theorist by heart. Financial support during the six months in Aachen was provided by Deutsche Forschungsgemeinschaft through the Schwerpunktprogramm "Sensomotorische Integration".

Michael Herrmann introduced me to Hans-Ulrich Bauer and thus paved my way to the cradle of quantum mechanics. The project I was originally hired for-information theoretic analysis of the neural code - soon turned stale. I am grateful to Hans-Ulrich Bauer and Theo Geisel for leaving me the freedom to return to and expand on my earlier work on stochastic resonance in that situation.

Theo Geisel has created a most extraordinary and stimulating research environment at the Max-Planck-Institut für Strömungsforschung. I cannot 
imagine another Doktorvater who would have permitted me to work as independently as he did, and would have encouraged outside contacts and collaborations so generously, not to speak of numerous national and international meetings I was able to attend.

It has been a pleasure to have Steffen Arnrich, Rita Bartels, Matthias Bethge, Dmitri Bibitchkov, Agnes Bleile, Ragnar Fleischmann, Lars Hufnagel, Matthias Kaschube, Roland Ketzmerick, Tsampikos Kottos, Karsten Kruse, Stefan Liehr, Norbert Mayer, Klaus Pawelzik, Frédéric Piéchon, Alexander Ossipov, Verena Rosenthal-Pilz, Holger Schanz, Dennis Springsguth, Frank Steinbach, Marc Timme, and Matthias Weiß as colleagues here in Göttingen. I enjoyed the collaboration with Wolf Dieter Brandt as system administrator, who kept our workstation cluster running non-stop in spite of persistent user interference. Corinna Trautsch did a great job in keeping chaos organized in a cheerful mood. I would like to thank Fred Wolf for some inspiring discussions, Udo Ernst and Michael Herrmann for organizing the WENGEN '98 neuroworkshop in Clausthal-Zellerfeld, and Silke Dodel for putting up with my eyes' craving for shadow in the afternoons. Dirk Brockmann creates simply the best websites in town. Helmut Eckelmann and Ursula Eberhardt advised me on the inner workings of Göttingen's doctorate examinations.

Financial support since August 1997 has been provided by Deutsche Forschungsgemeinschaft through Sonderforschungsbereich 185 (Nichtlineare Dynamik), the Leibniz-Preis to Theo Geisel, and the Max-Planck-Institut für Strömungsforschung.

Beyond Göttingen, the collaboration with Wulfram Gerstner, Lausanne, has been very stimulating. I am particularly indebted to him for suggesting to test and improve the hazard models. Frank Moss' excitement about my work has been a tremendous encouragement. Thanks are due to Alix Herrmann and Winfried Wojtenek for inviting me to Lausanne and St. Louis, respectively. Discussions with Katrin Suder, Bochum, Tony Burkitt, Melbourne, Sonja Grün, Frankfurt, and Ad Aertsen and his group in Freiburg have been inspiring throughout the years. Hans A. Braun, Marburg, kindly provided me with the orignial of Figure 5.11.

I would like to thank Reiner Kree for being my second referee.

Thale Wester made sure I started writing in time and made valuable suggestions on the introduction. Fred Wolf and Markus Diesmann have been exacting proofreaders, who kept embarrassing mistakes from seeing the light of day.

My parents, my brother and sister, and Thale have contributed to the genesis of this work through their loving support in more wonderful ways than a page can hold. I dedicated this thesis to them. 


\title{
Curriculum Vitae
}

\author{
January 6, 1968 born in Jülich \\ Nationality German \\ 1974-1978 Emschertal-Grundschule, Dortmund \\ 1978-1987 Gymnasium an der Schweizer Allee, Dortmund \\ 1984/85 Concord Academy, Concord, MA, USA \\ 1987 Abitur \\ 1987-1989 Civil Service \\ 1989-1995 Student of physics and mathematics, RWTH Aachen \\ 1992-1996 Stipend of Studienstiftung des deutschen Volkes \\ 1995 Diploma in physics, RWTH Aachen \\ 1995-1996 Laboratory for Neural Modeling, Frontier Research \\ Program, RIKEN, Wako, Japan \\ 1997 Institut für Biologie II, RWTH Aachen \\ 1997- Max-Planck-Institut für Strömungsforschung, \\ Göttingen
}


